\title{
Combinatorial Real-Time High Throughput Analyses of Cell Behavior Upon Exposure to Analytes
}

\author{
Reem Eldawud
}

Follow this and additional works at: https://researchrepository.wvu.edu/etd

\section{Recommended Citation}

Eldawud, Reem, "Combinatorial Real-Time High Throughput Analyses of Cell Behavior Upon Exposure to Analytes" (2016). Graduate Theses, Dissertations, and Problem Reports. 5535.

https://researchrepository.wvu.edu/etd/5535

This Dissertation is protected by copyright and/or related rights. It has been brought to you by the The Research Repository @ WVU with permission from the rights-holder(s). You are free to use this Dissertation in any way that is permitted by the copyright and related rights legislation that applies to your use. For other uses you must obtain permission from the rights-holder(s) directly, unless additional rights are indicated by a Creative Commons license in the record and/ or on the work itself. This Dissertation has been accepted for inclusion in WVU Graduate Theses, Dissertations, and Problem Reports collection by an authorized administrator of The Research Repository @ WVU.

For more information, please contact researchrepository@mail.wvu.edu. 


\title{
Combinatorial Real-Time High Throughput Analyses of Cell Behavior Upon Exposure to Analytes
}

\author{
Reem Eldawud
}

Dissertation submitted to the Benjamin M. Statler College of Engineering and Mineral Resources at West Virginia University

In partial fulfillment of the requirements for the degree of Doctor of Philosophy

in the Chemical and Biomedical Engineering Department

Dr. Cerasela Zoica Dinu, Committee Chairperson

Dr. Rakesh Gupta

Dr. Charter Stinespring

Dr. Yon Rojanasakul

Dr. Todd Stueckle

Department of Chemical and Biomedical Engineering

Morgantown, West Virginia

2016

Keywords: Real-time, Cell sensing, Carbon nanotubes, Anti-cancer, Nanodiamonds

Copyright 2016 Reem Eldawud 


\section{Abstract \\ Combinatorial Real-Time High Throughput Analyses of Cell Behavior Upon Exposure to Analytes}

\section{By Reem Eldawud}

The majority of current techniques to assess the toxicity of analytes (i.e., nanomaterials, drugs and toxins) in vitro rely on the application of affinity or catalytic recognition elements (i.e., biosensors), their activity, sensitivity and selectivity, as well as the processing power of micro- and opto-detection devices. For instance, water-soluble tetrazolium (WST-1) assay is among the most common techniques used to measure the viability of cells following exposure. Specifically, in this assay a catalytic biosensor (tetrazolium salt) is reduced by cellular $\mathrm{NAD}(\mathrm{P}) \mathrm{H}$-dependent oxidoreductase or mitochondrial dehydrogenases resulting in colorimetric changes quantified using a UV-Vis spectrophotometer (detection element). Similarly, imaging of cellular components mainly relies on the application of affinity biosensors that bind to selected cellular organelles or proteins and subsequently emit a fluorescent signal upon detection using fluorescence microscopy.

Although, the majority of these assays and techniques have high sensitivity, stability and reliability, inaccurate measurements and false positives may occur especially when detecting the toxicity of nanoparticles, such as carbon nanotubes (CNTs). In principle, the high surface area of CNTs as well as the presence of metal impurities and functional groups on their external surfaces decrease the availability and the activity of biosensors, as well as interfere with the detection ability of micro-and optical processors. Therefore, there is a critical need for sensitive bio-sensing tools to provide comprehensive measurements of the cellular behavior post exposure to analytes (e.g., nanomaterials, drugs and toxins) without relying on the applications of biosensors. 
In this work a combinatorial approach had been employed to evaluate the cellular behavior and fate in real-time using an Electric Cell Impedance Sensing (ECIS). In principle, the ECIS system relies on the natural sensitivity of cells to function as a primary transducer to provide high throughput, real-time behavioral measurements upon exposure to different analystes.

The ECIS system has been previously employed to quantify changes in cell morphology, cell adhesion, cell migration, chemotaxis, and wound healing capability, in response to different drugs, toxins and chemotherapeutic agents or just for standard behavior quantification. Herein, we have extended on these studies by employing the ECIS system to evaluate the cellular behavior of human lung (immortalized and non small lung cancer) cells upon exposure to different concentrations and types of nanoparticles and drugs. The ECIS real-time measurements were further complemented and correlated with different cellular and microscopical assays (Western blots, flow cytometry, optical and florescence imaging) to derive structure-function relationships and thus unravel the cellular mechanisms associated with any cytotoxic or apoptotic events induced upon analytes exposure.

Chapter one presents a systematic study of the cellular behavior of human lung epithelial cells upon exposure to single walled carbon nanotubes (SWCNTs) with user-defined physicochemical properties. Specifically, SWCNTs implementation in biomedical application has been hindered by many uncertainties regarding their toxicity and fate inside biological systems. Previous reports have attributed SWCNTs' toxicity to their length, surface chemistry, aggregation or metal impurities to name a few. However, due to the large variety of SWCNTs, the numerous modification techniques and current limitations in standardized toxicity assays, there is a discrepancy in literature and no mechanistic correlation between the changes in the cellular behavior and SWCNTs physico-chemical properties. In this chapter, we provide a comprehensive 
analysis of the cellular behavior, interactions and fate upon exposure to different concentrations and functionalities of SWCNTs. (Publication: Reem Eldawud, Alixandra Wagner, Chenbo Dong, Yon Rojansakul, Cerasela Zoica Dinu, Electronic platform for real-time multi-parametric analysis of cellular behavior post-exposure to single-walled carbon nanotubes, Biosensors and Bioelectronics, Volume 71, September 2015, Pages 269-277).

Chapter two provides comprehensive analysis of the potential applicability of nanodiamonds (NDs) in the development of the next generation of bionanotechnological products to be used for drug and gene delivery, bio-imaging and biosensing. In this chapter, we hypothesized that the high NDs biocompatibility is a result of their reduced ability to induce membrane disruption or damage during cellular translocation. Specifically, in this chapter we used multi-scale combinatorial approaches to simulate and evaluate NDs interactions with the cellular plasma membrane as well as their over-all effects on cell fate. (Publication: Reem Eldawud, Manuela Reitzig, Jörg Opitz, Yon Rojansakul, Wenjuan Jiang, Shikha Nangia and Cerasela Zoica Dinu, Combinatorial approaches to evaluate nanodiamond uptake and induced cellular fate, Nanotechnology, Volume 27, Number 8, January 2016).

Chapter three provides a systematic investigation of the potential anti-cancer mechanisms associated with cellular exposure to digitoxin at subtherapeutic, therapeutic and toxic concentrations. Specifically, Digitoxin belongs to a naturally occurring class of cardiac glycosides (CG) known for its anti-cancer effects against non-small lung cancer cells (NSCLC). However, concerns associated with its narrow therapeutic index and its concentration-dependent mechanism of action are rising. Thus, before digitoxin potential implementation in designing and developing safer and more effective CG-based anti-cancer therapies, its pharmacological and safety profiles need to be fully elucidated. In this chapter, the ECIS system was employed to provide a systematic 
investigation of the potential anti-cancer effects associated with digitoxin exposure on non-small lung cancer cells (NSCLC) in a time and dose-dependant manner. (Publication: Reem Eldawud, Todd Stueckle, Surya Manivannan, Hossam Elbaz, Michael Chen, Yon Rojanasakul and Cerasela Zoica Dinu, Real-time analysis of the effects of toxic, therapeutic and sub-therapeutic concentrations of digitoxin on lung cancer cells, Biosensors and Bioelectronics, Volume 59, September 2014, Pages 192-199).

This thesis has two chapter appendices that further investigate the effects of purified multiwalled carbon nanotubes exposure on human lung epithelial cells using combinatorial methods that employ fluorescent activated cells sorting and atomic force microscopy as well as conventional toxicity assays. (Chenbo Dong*, Reem Eldawud*, Linda M. Sargent, Michael L. Kashon, David Lowry, Yon Rojanasakul, and Cerasela Zoica Dinu, Towards elucidating the effects of purified MWCNTs on human lung epithelial cells, Environmental Science Nano, Volume 1, Issue 6, August 2014, 595-603)- and (Chenbo Dong*, Reem Eldawud*, Linda M. Sargent, Michael L. Kashon, David Lowry, Yon Rojanasakul, and Cerasela Zoica Dinu, Carbon Nanotube Uptake Changes the Biomechanical Properties of Human Lung Epithelial Cells in a Timedependent Manner, Journal of Material Chemistry B, Volume 1, Issue 3, April 2015, 39833992). * Equal contribution authors. 


\section{Dedication}

To my parents Zahi and Haifa Eldawud My sister Dina and brother Dawud Eldawud 


\section{Acknowledgement}

First and Foremost, I would like to express my sincere gratitude and thanks to my advisor Prof. Cerasela Zoica Dinu for her continuous support of my Ph.D study and research, as well as her constant advice and assistance. Her encouragement and guidance on my research as well as my career choices have been priceless. I must thank her for everything that has now become possible in my future career, my experience at WVU would not have been as enriching without her guidance and mentorship. I doubt that I will ever be able to convey my appreciation fully, but I owe her my eternal gratitude.

I also would like to thank Prof. Yon Rojanasakul whose collaborative efforts have made my research a possibility. A special thanks to Dr. Todd Stueckle for his guidance and continuous advice during my studies. I would also like to thank my committee members; Prof. Rakesh Gupta and Prof. Charter Stinespring for serving as my committee members even at hardship.

I would like to thank the current and former members of Prof. Dinu's and Prof. Rojanasakul's lab groups with whom I have worked closely over the years. I also want to thank the staff scientists and faculty of the Chemical Engineering and the Pharmaceutical Sciences Departments at WVU for their help and support. Also a special thanks to Dr. Kimberly Quedado for her continuous guidance, encouragement and support. I am also very appreciative to all the support, encouragement and advice I have received from Prof. Fonda Holehouse and David Ramsburgh, you have opened so many doors and opportunities for me.

I also have to thank my family and friends who have always been there for me and supported me in everything I have done. 


\section{Table of Contents}

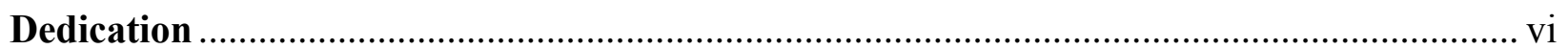

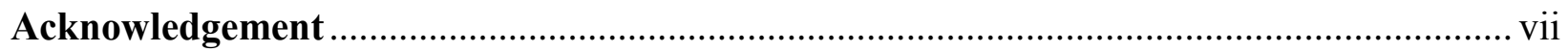

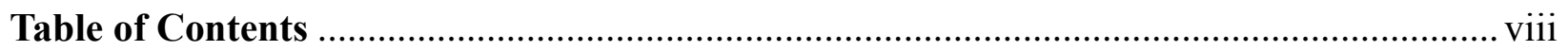

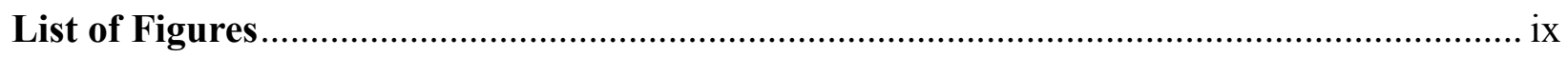

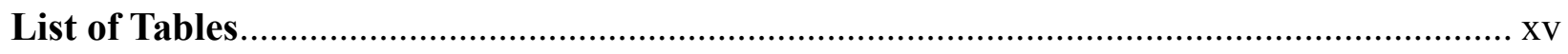

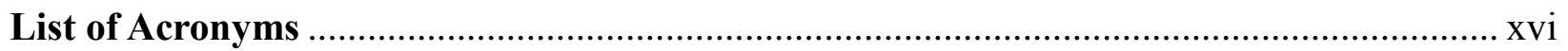

Chapter 1: Electronic Platform for Real-time Multi-parametric Analysis of Cellular Behavior Post

Exposure to Single-Walled Carbon Nanotubes..................................................................... 1

Chapter 2: Combinatorial Approaches to Evaluate Nanodiamonds Uptake and Induced Cellular

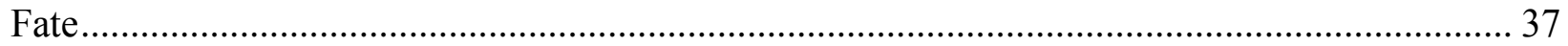

Chapter 3: Real Time Analysis of the Effects of Toxic, Therapeutic and Sub-therapeutic

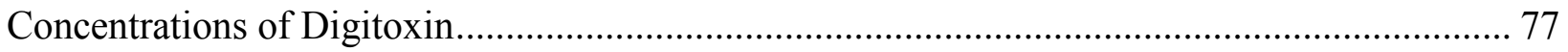




\section{List of Figures}

\section{Chapter 1: Electronic Platform for Real-time Multi-parametric Analysis of Cellular Behavior Post Exposure to Single-Walled Carbon Nanotubes}

Figure 1: Characterization of the chemical and physical properties SWCNTs. a) Raman spectra; b) Elemental composition; and c) Length and agglomerate size analysis of the SWCNT samples function of the treatment time. (* Indicates significant difference between the pristine and acid treated SWCNTs, while $¥$ indicates a significant difference between the 3 and $6 \mathrm{~h}$ acid treated SWCNTs respectively, $\mathrm{p}<0.05)$.

Figure 2: Representative real-time measurements of the normalized resistance showing changes in the cellular behavior from inoculation until the formation of a confluent monolayer (a) and subsequently $48 \mathrm{~h}$ following exposure to b) pristine, c) $3 \mathrm{~h}$ treated and d) $6 \mathrm{~h}$ acid treated SWCNTs.

Figure 3: Real-time measurements of the normalized alpha parameter following exposure to a) pristine, b) $3 \mathrm{~h}$ and c) $6 \mathrm{~h}$ acid treated SWCNTs for $48 \mathrm{~h}$. d) Cross-comparison in alpha parameter following 24 and $48 \mathrm{~h}$ exposure to the different SWCNTs samples. (* Indicates significant difference between the alpha values of the cells exposed to SWCNTs and control cells; ** indicates significant difference between the alpha value after 24 and $48 \mathrm{~h}$ of exposure, $p<0.05$ ).

Figure 4: Percentage of apoptotic cells following 24 and $48 \mathrm{~h}$ exposure to different concentrations of pristine, 3 and $6 \mathrm{~h}$ acid treated SWCNTs. (* Indicates significant differences between SWCNTs exposed cells and control cells, $p<0.05)$.

Figure 5: Schematic of the SWCNT-induced cellular changes as a reflection of changes in 
cellular morphology, cell-cell interactions, and cellular attachment properties. Those changes are analyzed in relation to the SWCNT physico-chemical characteristics to determine nanotubes internalization and induced cellular fate. 


\section{Chapter 2: Combinatorial Approaches to Evaluate Nanodiamonds Uptake and Induced Cellular Fate}

Figure 1: Physico-chemical characterization of detonation nanodiamonds (NDs), (a) Fourier Transform Infrared spectra indicating the presence of O-rich groups. (b) Transmission and Scanning Electron microscope images (TEM and SEM in insert respectively) showing the planner structure and the primary agglomerates formed from NDs. (c) Dynamic light scattering (DLS) intensity distribution showing the average agglomerate diameter of NDs in water and cellular media.

Figure 2: ND uptake and induced cellular biocompatibility following NDs exposure. (a) Normalized FITC signal of cells exposed to $(25,50,100,200,400 \mu \mathrm{g} / \mathrm{ml})$ NDs for 1, 2, 3 and 24 $\mathrm{h}$ respectively. (b) Representative normalized resistance measurements of BEAS-2B cells upon their exposure to different concentrations of NDs, (c) Quantifications of membrane fluctuations of cells exposed to NDs relative to unexposed cells. Differences were considered statistically significant for $\mathrm{p}^{*}<0.05$.

Figure 3: (a) Viability analysis following exposure to NDs for $24 \mathrm{~h}$ relative to control (unexposed) cells. (b) Cell cycle analysis following $24 \mathrm{~h}$ exposure to NDs. All differences were considered statistically significant for $\mathrm{p}^{*}<0.05$.

Figure 4: Coarse-grained (CG) computational analysis of the potential of mean force (PMF) profiles for the $3 \mathrm{~nm}(\mathrm{a}), 5 \mathrm{~nm}(\mathrm{~b})$ and $7 \mathrm{~nm}$ (c) NDs as function of the distance between the center-of-mass (COM) of NDs and membrane mid-plane. 
Figure 5: Snapshots revealing the translocation of NDs with different diameter (i.e., 3, 5 and $7 \mathrm{~nm}$ respectively) through the membrane lipid bilayer. The images showed the membrane disruption in response to the NDs. 


\section{Chapter 3: Real Time Analysis of the Effects of Toxic, Therapeutic and Sub-therapeutic Concentrations of Digitoxin}

Figure 1: (A) Fluorescent images of Hoechst stained NCI-H460 cells 24 h post-exposure to digitoxin. Cells treated with 40 and $80 \mathrm{nM}$ digitoxin showed major changes in their nuclei morphologies (white circles) relative to controls or cells treated with 10 and $25 \mathrm{nM}$ digitoxin. (B) Percentage of apoptotic cells upon exposure to digitoxin is dose and time-dependent. Difference is considered significant for ${ }^{*} p<0.05$.

Figure 2: (A) Live cell counts after 24, 48 and $72 \mathrm{~h}$ exposure to different concentrations of digitoxin. (B) Western blot analysis of CDK4 expression relative to control $\beta$-actin $24 \mathrm{~h}$ postexposure to digitoxin. (C) Quantification of CDK4 expression levels relative to $\beta$-actin. A significant difference is indicated for $* p<0.05$.

Figure 3: (A) Schematic illustration of an ECIS array, with a demonstration of the ECIS current pathways accounting for the resistance measurements. (B) Representative real-time measurements of the normalized resistance mathematically modeled according to the growth and spreading behavior of the NCI-H460 cells over the gold electrodes; the standard deviation is shown every two and a half hours and represents an average of 16 measurements ( 2 arrays with 8 wells each). (C) Real-time changes in $\alpha$ parameter.

Figure 4: (A) Representative analysis of the real-time behavior of NCI-H460 cells following digitoxin exposure. (B) Changes in $\alpha$ parameter post-exposure to digitoxin in real-time; * signifies changes considered significant $p<0.05$ 
Figure 5: Proposed cellular signaling pathways and regulatory effects between cellular proliferation and adhesion upon exposure to sub-therapeutic concentrations of digitoxin. Sequestration of CDK4 in cytoplasm by association with ZONAB regulates cell's junction dynamics. 


\section{List of Tables}

Chapter 2: Combinatorial Approaches to Evaluate Nanodiamonds Uptake and Induced Cellular Fate

Table 1: Calculated barrier heights, rate constants and half-lives for cellular uptake of NDs without BSA protein corona 


\section{List of Acronyms}

- $\quad$ AFM: Atomic force microscopy

- ANOVA: Analysis of variance

- ATCC: American Type Culture Collection

- BCA: Bicinchoninnic Acid Assay

- BEAS-2B: Immortalized human lung epithelial cells

- $\quad$ BSA: Bovine Serum Albumin

- $\quad$ CB: Carbon Black

- CDK4: Cyclin Dependent Kinase-4

- $\quad$ CG: Cardiac Glycosides

- CG: Coarse Grained

- CNTs: Carbon nanotubes

- $\quad$ COM: Center of-Mass

- DLS: Dynamic Light Scattering

- DMEM: Dulbacco Minimum Essential Media 
- DMSO: Dimethyl Sulfoxide

- DSPC: Distearoylphosphatidyl Choline

- DSPG: Distearoylphosphatidyl Glycerol

- ECIS: Electrical Cell Impedance Sensing

- EDTA: Ethylene Diaminetetracetic Acid

- EDX: Energy Dispersive X-Ray Spectroscopy

- FACS: Fluorescence Activated Cell Sorting

- FBS: Fetal Bovine Serum

- FSC: Forward Scatter

- FTIR: Fourier Transform Infrared Spectroscopy

- $\quad \mathrm{HCl}$ : Tris-Hydrochloric Acid

- MAPK: Mitogen Activated Protein Kinase

- $\quad \mathrm{NaCl}$ : Sodium Chloride

- NDs: Nanodiamonds

- NPT: Isobaric Isothermal 
- $\quad$ NSCLC: Non-small lung cancer cells

- NVT: Isochoric Isothermal

- OSHA: Occupational Safety and Health Administration

- PBS: Phosphate Buffer Saline

- PI: Propidium Iodide

- PI3K : Phosphatidylinositol-3-Kinase

- PMF: Potential of Mean Force

- PVDF: Polyvinylidene Fluoride (PVDF)

- QD: Quantum Dots

- RPMI-1640: Roswell Park Memorial Institute-1640 medium

- SDS: Sodium Dodecyle Sulfate

- SEM: Scanning Electron Microscope

- $\quad$ SSC: Side Scatter

- SWCNTs: Single-walled carbon nanotubes

- $\quad$ TBST: Tris-Buffered Saline 
- TEM: Transmission Electron Microscope

- WST-1: Water Soluble Tetrazolium

- ZO-1: Zona Occludin-1 


\title{
Chapter 1
}

\section{Electronic Platform for Real-time Multi-parametric Analysis of Cellular Behavior Post Exposure to Single- Walled Carbon Nanotubes}

\begin{abstract}
Single-walled carbon nanotubes (SWCNTs) implementation in a variety of biomedical applications from bioimaging, to controlled drug delivery and cellular-directed alignment for muscle myofiber fabrication, has raised awareness of their potential toxicity. Nanotubes structural aspects which resemble asbestos, as well as their ability to induce cyto and genotoxicity upon interaction with biological systems by generating reactive oxygen species or inducing membrane damage, just to name a few, have led to focused efforts aimed to assess their risk prior user implementation. In this study, we employed a non-invasive and real-time electrical cell impedance sensing (ECIS) platform to monitor cellular behavior of lung epithelial cells upon exposure to a library of SWCNTs with user-defined physico-chemical properties. Using the natural sensitivity of the cellular biosensors, we assessed SWCNT-induced cellular changes in relation to cell attachment, cell-cell interactions and cell viability. Our methods have the potential to lead to the development of standardized assays for risk assessment of other nanomaterials as well as risk assessment differentiation based on the nanomaterials surface chemistry, purity and agglomeration rate.
\end{abstract}




\section{Introduction}

Nanomaterials implementation in a variety of fields from microelectronics [1], to photooptics [2], aerospace [3], energy [4], sensors [5], bioimaging [6], and drug delivery [7] has raised awareness of their occupational safety and health-posed issues [8, 9]. Current available techniques to assess in vitro toxicity of nanomaterials such as silica [10], silver nanoparticles [11], carbon[12] or metal-oxide-based nanomaterials [13], rely on the functionality, affinity and/or selectivity of a biological recognition elements (e.g., biosensor, antibodies, cellular membrane, organelles or DNA etc.) as well as the processing power and detection capability of micro and opto-electronics $[14,15]$. Such techniques record induced changes to single or a population of cells (for instance the generation of reactive oxygen species (ROS) following exposure to silver nanoparticles [16] or the changes in cellular viability and proliferation post exposure to gold [17] or titanium dioxide nanoparticles [18] etc.) at discrete and user-controlled time points (e.g., 12, 24 or $48 \mathrm{~h}$ ) mainly through invasive, laborious and costly assays that require intensive and time sensitive manipulation and handling of the samples [19, 20].

Recently it was however found that some of these techniques are less applicable and reliable for assessing toxicity of carbon nanotubes (CNTs), fullerenes $\left(\mathrm{C}_{60}\right)$, carbon black $(\mathrm{CB})$, and quantum dots (QD) [21, 22]. For instance, results showed that CNTs' high surface area, high adsorption capability, high catalytic activity and their characteristic optical properties could interfere with the reagent used in detection and affect its emission capability [21, 23, 24]. Specifically, several studies showed that the suitability and accuracy of assays relying on catalytic and affinity biosensors such tetrazolium salt and neutral red [22] to measure cellular viability become questionable in the case of CNTs due to the adsorption or binding affinity of the reagent onto the CNT surfaces [21, 23, 24]. Such limitations in the current CNT-induced hazard 
assessments techniques [21] as well as the continuous development of CNT-based forms and shapes with various functionalities and physico-chemical properties [25, 26] do not allow for highthroughput and efficient toxicity assessment to be standardized and thus lead to minimum regulations of these nanomaterials exposure limits. Specifically, according to Occupational Safety and Health Administration (OSHA), CNT exposures currently fall under the category of "particles not otherwise regulated" at a limit concentration of $5 \mathrm{mg} / \mathrm{m}^{3}$ particles $[27,28]$. If CNTs are to reach their full potential for biotechnological applications, in particular for the development of bioimaging probes and drug delivery platforms [7], then new and scalable methods that allow for accurate cyto and genotoxicity evaluations need to be developed and implemented in real-time, all with minimum false positives. Such methods should also be able to allow risk assessment analysis with the different concentrations of nanomaterial used for exposure, as well as risk evaluation correlations based on the nanomaterial length [29], diameter [30], aggregation rate [31], metal impurities [32], and/or surface chemistry [33], just to name a few.

In this study, we implemented a rapid, non-invasive, high throughput, real-time continuous monitoring platform to detect CNT-induced changes in the behavior of confluent model human lung epithelial cells regularly used to investigate toxicity of nanomaterials $[16,34]$. Our approach used an electric cell impedance sensing (ECIS) device that allowed the cells immobilized onto gold electrodes to serve as a proxy for direct CNT risk assessment and differentiation based on nanotube physico-chemical properties. By using the natural sensitivity of the cell transducers, we convert biological activity into electrical measurements to reflect changes in cell attachment and cell-cell interactions in response to nanotube's characteristics. ECIS was previously employed to monitor cellular changes upon exposure to digitoxin [35] (a cardiac glycoside with anti-cancer potential), cytochalasin D (a cytoskeletal inhibitor) [36] or sodium arsenate (a toxin responsible 
for cell retraction and changes in cytoskeleton) [37], all under user-controlled conditions. Our experimental procedure not only capitalizes on bioengineering means to provide parallel analysis of the cellular behavior associated with exposure to a library of CNTs, all at a high-sensitivity, in real-time and with minimum sample invasion, but further overcomes the limitations and concerns associated with the CNT interactions with the biological sensing elements. Moreover, our analysis could be extended to identify toxicity of other nanomaterials in a high-throughput and non-invasive fashion thus extending the ability for standardizing nanomaterial risk evaluation.

\section{Materials and Methods:}

\section{Single-walled carbon nanotubes (SWCNTs) functionalization}

Acid treated single-walled carbon nanotubes (SWCNTs) were produced by liquid phase oxidation of commercial pristine SWCNTs purchased from Unidym Inc. Specifically, the pristine SWCNTs were treated in a mixture of 3:1 (V/V) concentrated sulfuric acid (Fisher Scientific, 96.4\%) and nitric acid (Fisher Scientific, 69.6\%) for different periods of time (i.e., 3 and 6 h) to obtain SWCNTs with different degree of O-related functionalities and lengths [38]. Upon time elapsed, the SWCNTs/acid mixture was diluted in deionized water, filtered (GTTP, $0.2 \mu \mathrm{m}$, Fisher Scientific) and washed extensively with deionized water to remove impurities or dissociated metal catalysts. 


\section{Characterization of pristine and acid treated SWCNTs}

Raman spectroscopy was used to investigate the physical and chemical properties of SWCNTs. For this, SWCNT powders (pristine or acid treated SWCNTs, both 3 and 6 h were assessed) were deposited onto clean glass slides and scanned using a Raman spectrometer (CL532$100,100 \mathrm{~mW}, \mathrm{USA}$ ) and a $532 \mathrm{~nm}$ green laser with a spot size of $<0.01 \mathrm{~mm}^{2}$ directed though a 50X objective. Detailed scans were taken in the 100 to $3200 \mathrm{~cm}^{-1}$ range; low energy laser (i.e., $<$ $0.5 \mathrm{mV})$ and short exposure time $(10 \mathrm{sec})$ were maintained to prevent unexpected heating effects of the samples.

Energy dispersive X-ray spectroscopy (EDX) was employed for quantitative elemental analysis of the SWCNTs. Dry samples (pristine and acid treated SWCNTs) were mounted onto silica wafers and their elemental composition was evaluated using a Hitachi S-4700 Field Emission Scanning Electron Microscope with a S-4700 detector integrating secondary (SE) and backscattered (BSE) electron detection (in a single unit).

Atomic Force Microscopy (AFM) was used in air tapping mode to investigate the lengths of pristine and acid treated SWCNTs [39]. Briefly, commercial Si tips (Asylum Research, AC240TS) were employed under their original manufacturing resonance frequency varying from

50 to $90 \mathrm{kHz}$. During the scanning process, the topography, phase and amplitude images of the SWCNT samples were collected simultaneously; a minimum of 3 scans were obtained for each SWCNT sample and at least 30 individual SWCNT were measured for an average of their length distribution.

Analysis of agglomerate size was performed using a dynamic light scattering device (DLS, DelsaTM). For this, suspensions of $50 \mu \mathrm{g} / \mathrm{ml}$ pristine and acid treated SWCNTs (both 3 and $6 \mathrm{~h}$ ) 
were prepared in Dulbacco Minimum Essential Media (DMEM) and analyzed at $20{ }^{\circ} \mathrm{C}$. For each sample, 150 measurements were recorded and the mean particle diameter was calculated by analyzing the intensity, volume, and number distribution data collected.

\section{Cell culture and SWCNTs exposure}

Immortalized human lung epithelial cells (BEAS-2B) purchased from American Type Culture Collection (ATCC) were cultured in DMEM medium supplemented with 5\% Fetal Bovine Serum (FBS), 2 mM L-glutamine and 100 units/ml penicillin/streptomycin (Invitrogen). Cells were passaged regularly and kept in $5 \% \mathrm{CO}_{2}$ at $37^{\circ} \mathrm{C}$. To prepare the pristine and acid treated SWCNTs (both 3 and $6 \mathrm{~h}$ ) for cellular exposures, the samples were first dispersed by sonication in deionized water to eliminate all large agglomerates (visual assessment). Subsequently, the samples were filtered using a $0.2 \mu \mathrm{m}$ pore filter membrane, resuspended in DMEM media with 5\% FBS, and again sonicated for 2 min to form stable dispersions.

\section{Electric cell impedance sensing (ECIS)}

Real-time quantification of cellular behavior post exposure to SWCNTs (pristine and acid treated) was performed using an electric cell impedance sensing device (ECIS, Applied Biophysics). In one set of experiments, two $8 \mathrm{~W} 10 \mathrm{E}+$ ECIS arrays with 40 gold electrodes each were simultaneously employed to provide concomitant measurements of 16 samples, all at multiple frequencies. Prior to each experiment, the gold electrodes were pre-equilibrated and stabilized for $3 \mathrm{~h}$ with $400 \mu \mathrm{l}$ of cellular media. Subsequently, BEAS-2B cells were seeded at a density of $2.5 \times 10^{5}$ cells $/ \mathrm{ml}$ into each well and allowed to settle and grow over the electrodes for $24 \mathrm{~h}$. Upon $24 \mathrm{~h}$, the cells were exposed to suspensions of 100, 50 and $25 \mu \mathrm{g} / \mathrm{ml}$ pristine and acid treated SWCNTs in cellular media and their behavior was continuously monitored for $48 \mathrm{~h}$. Using 
a multi-frequency recording mode, the resistance on the current passing in the spaces between the basal cell membrane and gold electrodes was recorded as the alpha parameter [35] to evaluate the cell adhesion characteristics and cell-substrate interactions [40, 41].

\section{Cell viability analysis}

BEAS-2B cells were seeded overnight onto a 12 -well plate at a density of $2.5 \times 10^{5}$ cells $/ \mathrm{ml}$ and exposed to suspensions of 100, 50 and $25 \mu \mathrm{g} / \mathrm{ml}$ pristine or acid treated SWCNTs (both 3 and $6 \mathrm{~h}$ were used) in cellular media for 24 and $48 \mathrm{~h}$ respectively. Subsequently, the cells were incubated with $10 \mu \mathrm{g} / \mathrm{ml}$ of Hoechst 33342 (Molecular Probes) for 30 min at $37{ }^{\circ} \mathrm{C}$ and then analyzed using fluorescence microscopy (Leica Microsystems) to assess the percentage of intensely condensed chromatin and/or fragmented nuclei. The apoptotic percentage was calculated as the percentage of cells with apoptotic nuclei over the total number of cells, both per field of view.

Cellular viability was investigated using Trypan Blue exclusion assay (Invitrogen). For this, BEAS-2B cells were seeded overnight onto a 12 -well plate at a density of $2.5 \times 105$ cells $/ \mathrm{ml}$ and exposed to 100,50 and $25 \mu \mathrm{g} / \mathrm{ml}$ pristine or acid treated SWCNT samples for 24 and $48 \mathrm{~h}$ respectively. Subsequently, the cells were washed twice with Phosphate Buffer Saline (PBS, Invitrogen), collected, stained with 0.4\% Trypan Blue dye at 1:1 volume ratio and counted using Countess $^{\mathrm{TM}}$ Automated Cell Counter (Invitrogen).

\section{Statistical analysis}

All results are presented as mean \pm standard deviation. Two-way analysis of variance (ANOVA) and unpaired two-tailed Student's T-test were performed using JMP 8.0 (SAS Institute) 
and SigmaPlot 10.0 (Systat Software Inc.). Viability experiments were performed 3 times with approximately 1,000 nuclei from ten random fields being analyzed for each sample, at every time point. ECIS experiments were performed in duplicates and repeated at least 3 times, for a minimum of 6 replicates per each sample. Results are considered significant when ${ }^{*} p<0.05$.

\section{Results}

\section{Preparation and characterization of a library of single-walled carbon nanotubes}

We prepared a library of single-walled carbon nanotubes (SWCNTs) with different physico-chemical properties by using liquid phase oxidation in a strong acid mixture of nitric and sulfuric acid for 3 and $6 \mathrm{~h}$ respectively [42]. The approach has been previously used to introduce O-containing functional groups, i.e., carboxyl, carbonyl groups, shorten the SWCNTs at the defect sites [43] and eliminate residual metal catalysts introduced during nanotube synthesis.

Raman resonance spectroscopy was used to characterize the structure, surface chemistry and degree of functionalization of the SWCNTs library, all as a function of the acid treatment period (Figure 1a). Results showed that the $\mathrm{G}$ band $\left(1590 \mathrm{~cm}^{-1}\right)$, mainly associated with atomic arrangement on the circumferential and axial direction of the SWCNT, did not undergo major changes upon acid treatment. In contrast, the $\mathrm{D}$ band (reflective of the sample disorder) became wider and shifted toward lower wavelengths, i.e., 1340.7 and 1338.1 for the 3 and $6 \mathrm{~h}$ treated SWCNTs respectively relative to $1353.50 \mathrm{~cm}^{-1}$ for pristine SWCNTs, suggesting that acid treatment altered the nanotubes surface chemistry and disrupted their well-structured walls. The intensity ratio between the $\mathrm{D}$ and $\mathrm{G}$ peaks $\left(I_{\mathrm{D}} / I_{\mathrm{G}}\right)$ for the $6 \mathrm{~h}$ acid treated SWCNTs was $0.47 \pm 0.056$ relative to the $I_{\mathrm{D}} / I_{\mathrm{G}}$ of $0.253 \pm 0.048$ for the pristine, while the $I_{\mathrm{D}} / I_{\mathrm{G}}$ of the $3 \mathrm{~h}$ acid treated SWCNTs was $0.21 \pm 0.102$. 
Energy Dispersive X-ray Spectroscopy (EDX; Figure 1b) was used to characterize the elemental composition of the samples upon acid treatment. Analysis showed that acid treatment changed the composition of the SWCNT samples by changing the percentage of $\mathrm{O}$ and $\mathrm{C}$ contents and reducing the percentage of metal impurities (i.e., $\mathrm{Fe}, \mathrm{Co}, \mathrm{Cl}$ ), all as a function of the acid treatment period.

Analysis of the average length and agglomerate size of pristine and acid treated SWCNTs were performed using an atomic force microscope (AFM) and dynamic light scattering technique (Figure 1c). Our results showed that upon $3 \mathrm{~h}$ acid treatment the average length of the pristine SWCNTs $(844 \pm 162 \mathrm{~nm})$ was reduced by $22 \%(656 \pm 207 \mathrm{~nm})$, while $6 \mathrm{~h}$ acid treatment led to $39 \%$ reduction $(517 \pm 208 \mathrm{~nm})$ respectively. Complementary, the average agglomerate size was reduced by $40 \%$ for the $3 \mathrm{~h}$ acid treated sample (from $615 \pm 133$ to $378 \pm 83 \mathrm{~nm}$ ) and by $22 \%$ (from $615 \pm 133$ to $481 \pm 95 \mathrm{~nm}$ ) for the $6 \mathrm{~h}$ acid treated nanotubes.

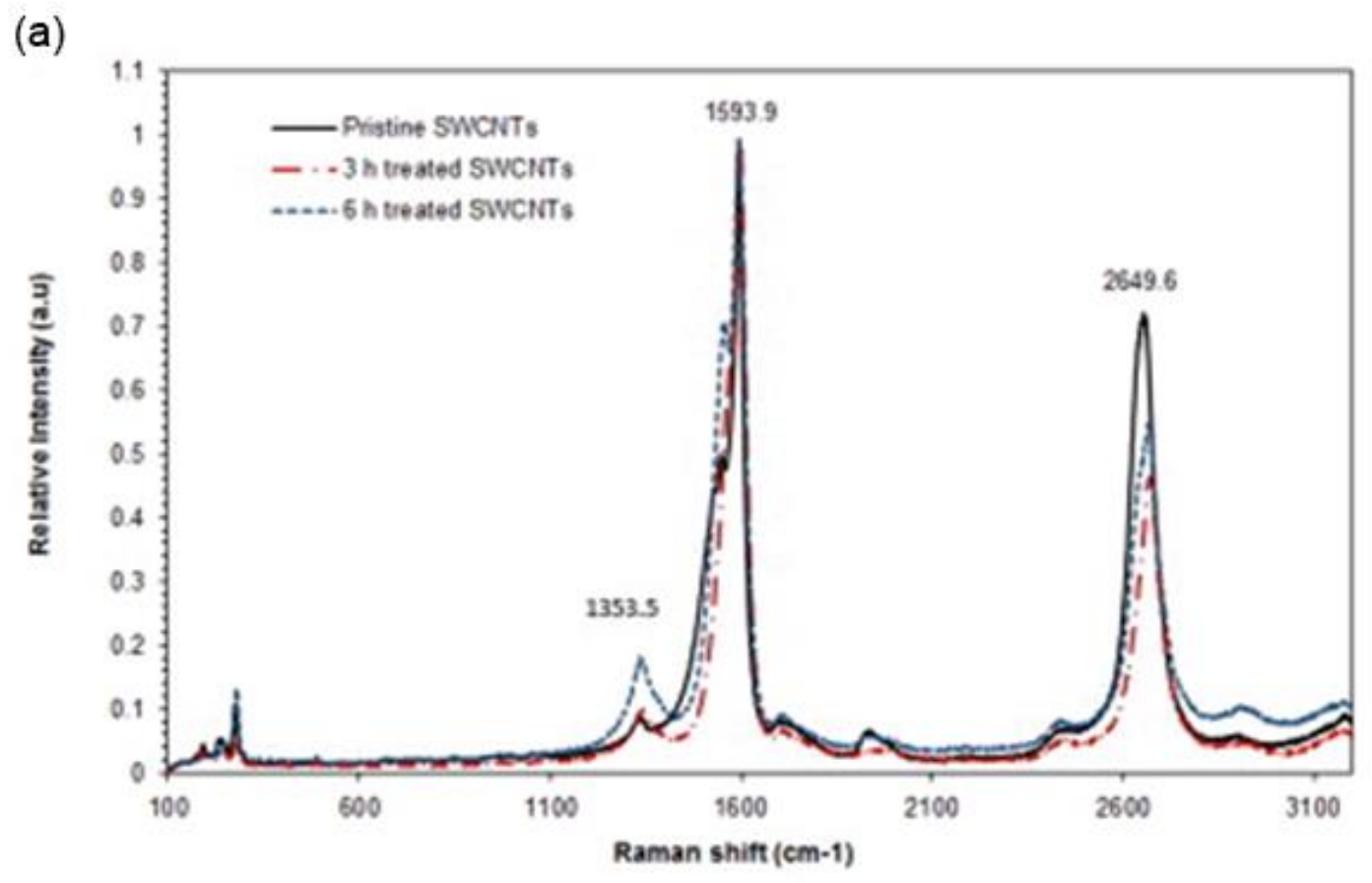



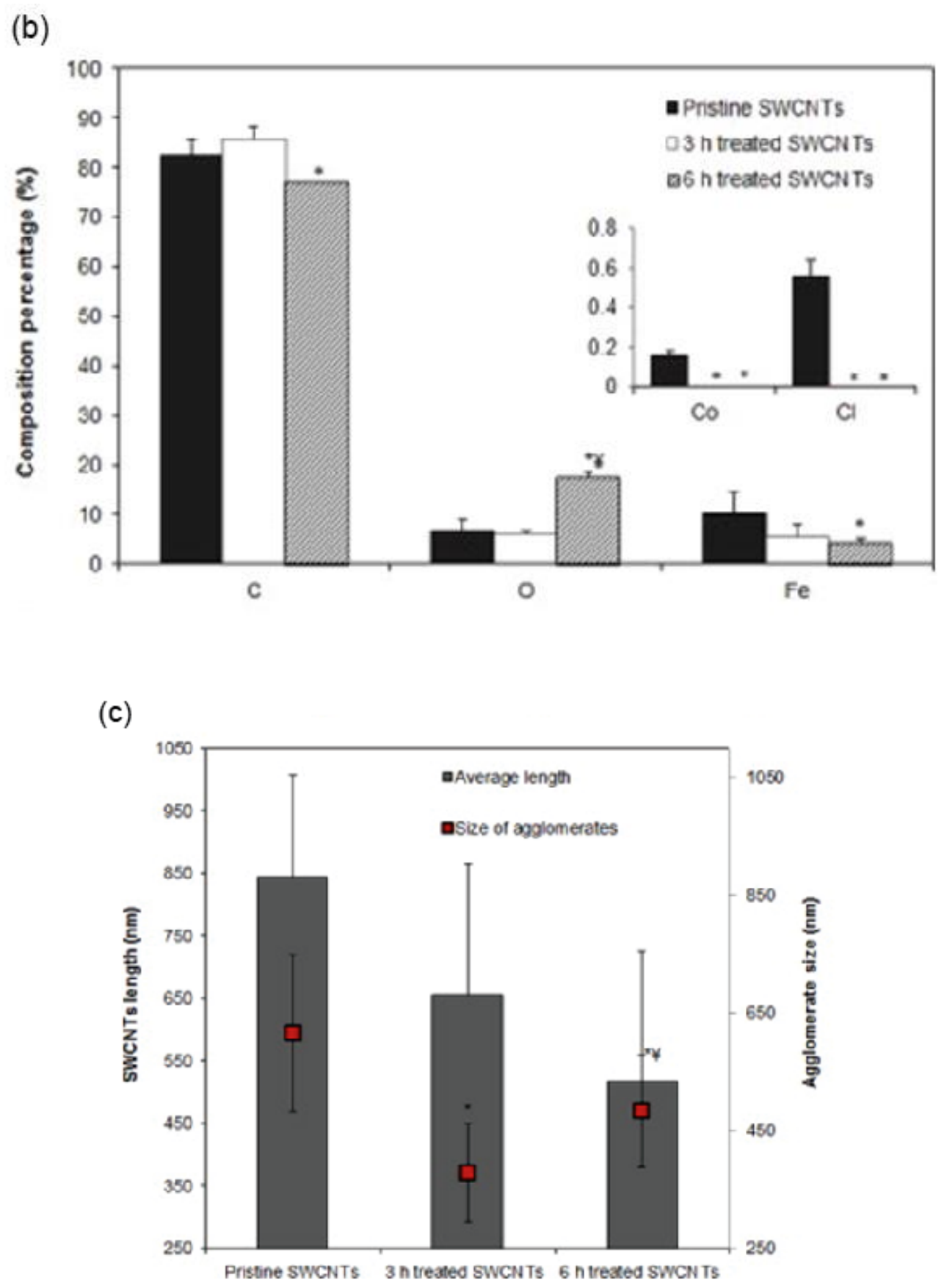

Figure 1: Characterization of the SWCNTs properties as a function of the treatment time. a) Raman spectra; b) Elemental composition; and c) Length and agglomerate size analysis (* Indicates significant difference between the pristine and acid treated SWCNTs, while $¥$ indicates a significant difference between the 3 and $6 \mathrm{~h}$ acid treated SWCNTs respectively, $\mathrm{p}<0.05$ ). 


\section{Real-time evaluation of cellular behavior upon exposure to SWCNTs with different physico- chemical properties}

The effects of the SWCNTs library on the behavior of model human lung epithelial cells (BEAS-2B) were assessed using electric cell impedance sensing (ECIS) by employing the natural sensitivity of the cells to provide high-throughput, parallel measurements of cellular attachment and cell-cell interactions [41, 44]. Briefly, BEAS-2B cells were seeded onto the ECIS electrodes and exposed to 100,50 and $25 \mu \mathrm{g} / \mathrm{ml}$ of either sample of SWCNTs (pristine, 3 or $6 \mathrm{~h}$ acid treated) for $48 \mathrm{~h}$. The exposure doses chosen for this investigation were extrapolated from in vivo studies mimicking acute human exposure levels at Occupational Safety and Health Administration (OSHA) for particles less than $5 \mu \mathrm{m}$ in diameter which correspond to workspace exposure at $5 \mathrm{mg} / \mathrm{m}^{3}$ from 8 years up to a lifetime span (32 years) $[45,46]$.

Representative measurements of the resistance values of cells from the time of inoculation to the formation of a confluent monolayer, and subsequently post-exposure to the pristine and acid treated SWCNTs are shown in Figure 2. Our results showed that upon cellular inoculation, the normalized resistance values increased in a time-dependent manner according to the settling, spreading and attachment behavior of the cells over the electrodes and are also dependent on the corresponding morphological changes. For instance, as the cells proliferate, divide, establish cellular connections and form a monolayer, their size, shape and morphology change in real-time, which in turn affect the restrictions on the ac current pathways and the corresponding resistance value [35].

Upon exposure to the library of SWCNTs, the resistance values have changed in with cells 
exposed to pristine SWCNTs showing significantly lower resistance than control cells, however with no significant changes in the values being observed for the different doses of exposure used in our experiments (Figure 2b).

Cells exposed to $3 \mathrm{~h}$ acid treated SWCNTs showed a reduction in the resistance value relative to the control cells; no significant differences between the different exposure doses were recorded however the highest drop in resistance was noticed for $100 \mu \mathrm{g} / \mathrm{ml}$ dose (Figure 2c). Cells exposed to $6 \mathrm{~h}$ acid treated SWCNTs also showed a drop in their resistance values relative to their control counterparts, all in a time and dose dependent manner, with a gradual increase in the normalized resistance values observed for the cells exposed to lower SWCNT doses (Figure 2d). Moreover, the results indicated that cells exposed to the $6 \mathrm{~h}$ acid treated SWCNTs at lower concentrations ( 50 and $25 \mu \mathrm{g} / \mathrm{ml}$ ) were able to overcome the disturbance caused by the exposure and regained their resistance values within $48 \mathrm{~h}$ from the time of exposure.

(a)

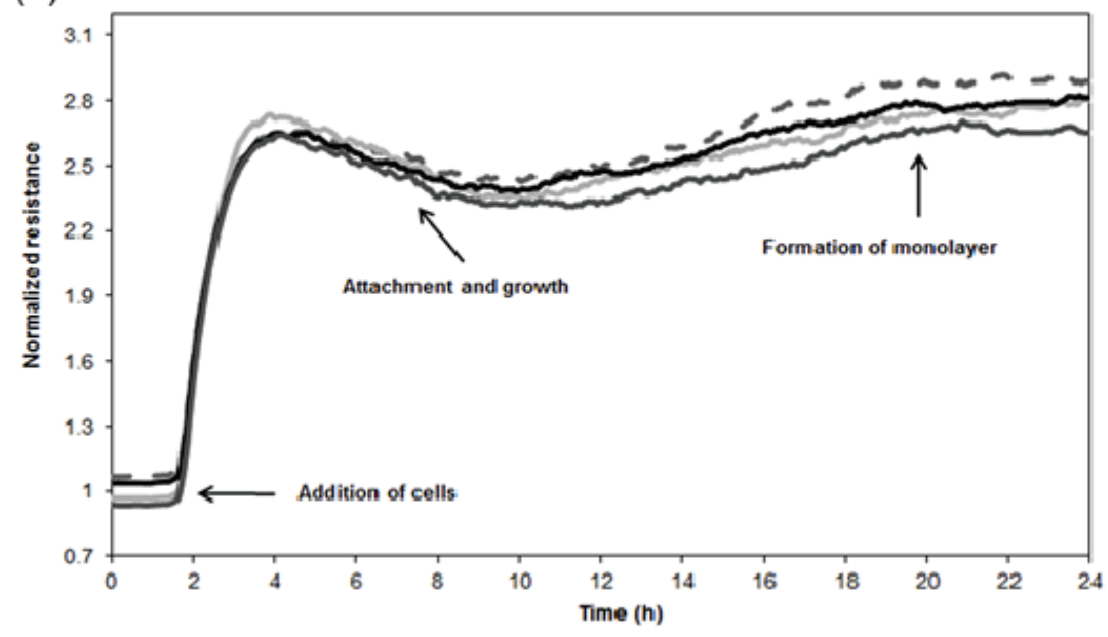


(b)

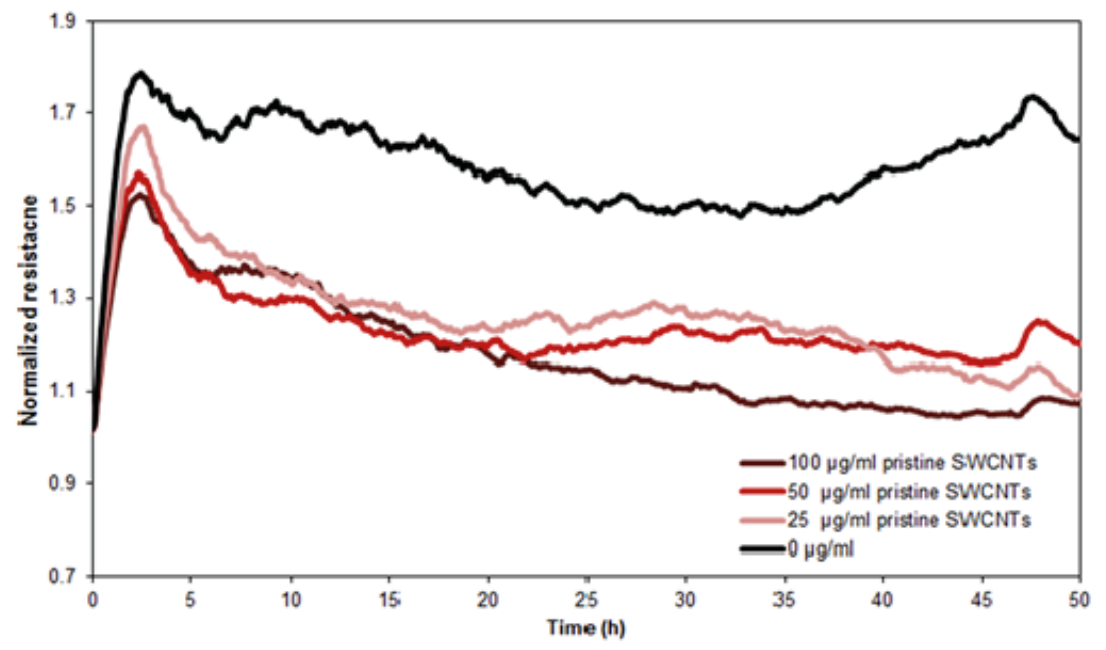

(c)

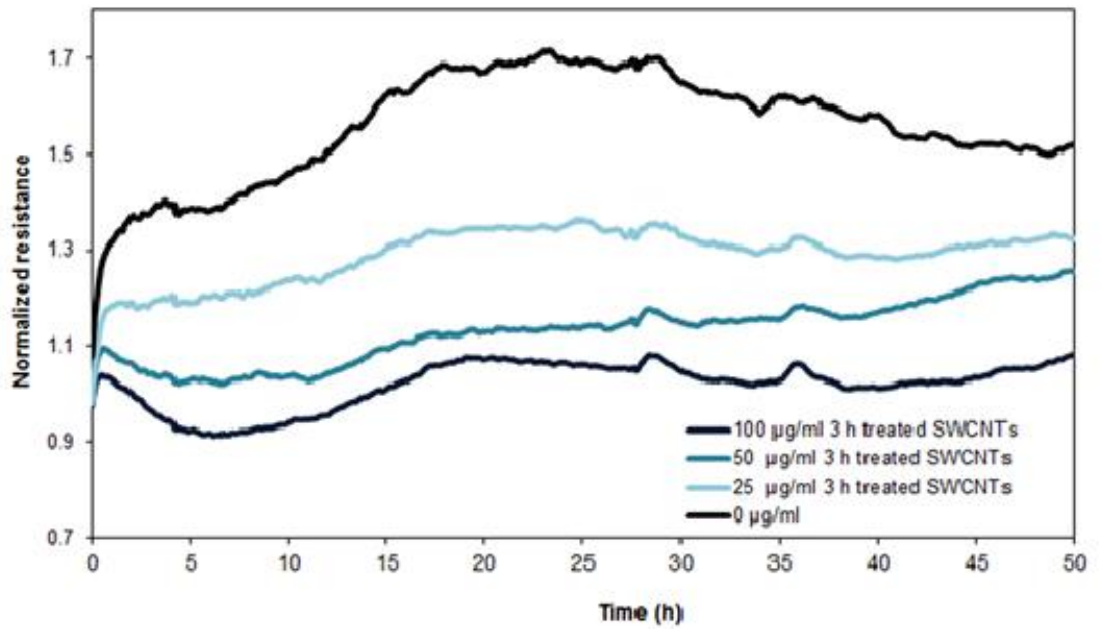

(d)

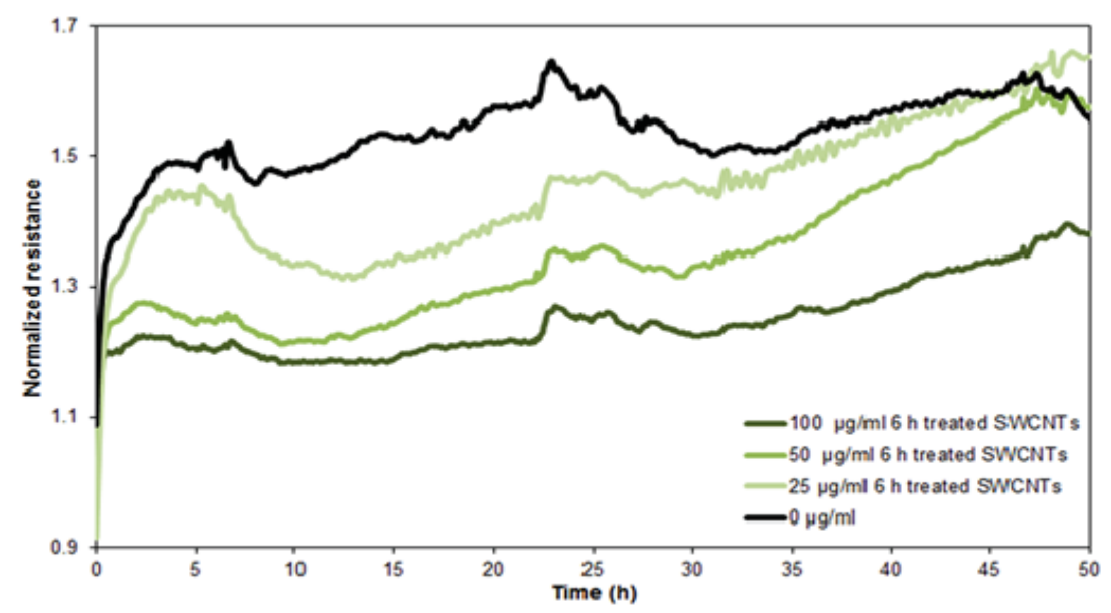


Figure 2: Representative real-time measurements of the normalized resistance showing changes in the cellular behavior from inoculation until the formation of a confluent monolayer (a) and subsequently $48 \mathrm{~h}$ following exposure to b) pristine, c) $3 \mathrm{~h}$ and d) $6 \mathrm{~h}$ acid treated SWCNTs.

Analysis of the different current pathways associated with the restrictions of the current flow in the spaces beneath the basal membrane of the attached cells onto the electrode substrates function of the characteristics of the SWCNTs library were also performed (Figure 3a). Reduction in alpha-parameter corresponds to an increase in the distance between the gold electrodes and the cell basal-membrane that will reflect loss of cellular adhesion $[35,41]$. Our results showed that alpha values for the cells exposed to SWCNTs were significantly lower than those of the control cells, with the differences being dependent on both the type of the nanotube and the exposure dose being used. Specifically, while the cells exposed to pristine SWCNTs showed a small reduction (Figure $3 \mathrm{~b}$ ), the cells exposed to $3 \mathrm{~h}$ acid treated SWCNTs showed a gradual decrease in their alpha which progressed both with time and the dose being tested (Figure 3c). Finally, cells exposed to 6 $\mathrm{h}$ acid treated SWCNTs exhibited the highest reduction in alpha relative to controls, however with no differences being observed between the different doses being tested.

Cross-comparison according to the different types of SWCNTs showed that exposure to the $6 \mathrm{~h}$ acid treated samples led to the highest dose-dependent reduction in cellular attachment relative to control cells. The reductions were dose-dependent with $60 \%, 68 \%$ and $72 \%$ change observed for cells exposed to 25, 50 and $100 \mu \mathrm{g} / \mathrm{ml}$ SWCNTs respectively (Figure 3d). Similarly, cellular exposure to $3 \mathrm{~h}$ acid treated SWCNTs led to $29 \%, 45 \%$ and $57 \%$ reduction in alpha after exposure to 25,50 and $100 \mu \mathrm{g} / \mathrm{ml}$ nanotube respectively. In contrast, cells exposed to pristine SWCNTs showed the lowest reduction in the cellular attachment (about 10\% less than the values of the control cells), with no significant differences being observed between the different exposure 
doses being investigated. Lastly, analysis showed that after $48 \mathrm{~h}$ the cells exposed to the $6 \mathrm{~h}$ acid treated SWCNTs were able to regain some of their adhesion characteristics by revealing an average increase of about $38 \%$ relative to their alpha values after $24 \mathrm{~h}$ of exposure. In contrast, the cells exposed to both $3 \mathrm{~h}$ acid treated and pristine SWCNTs continued to maintain a decrease in their cellular adhesion.

(a)

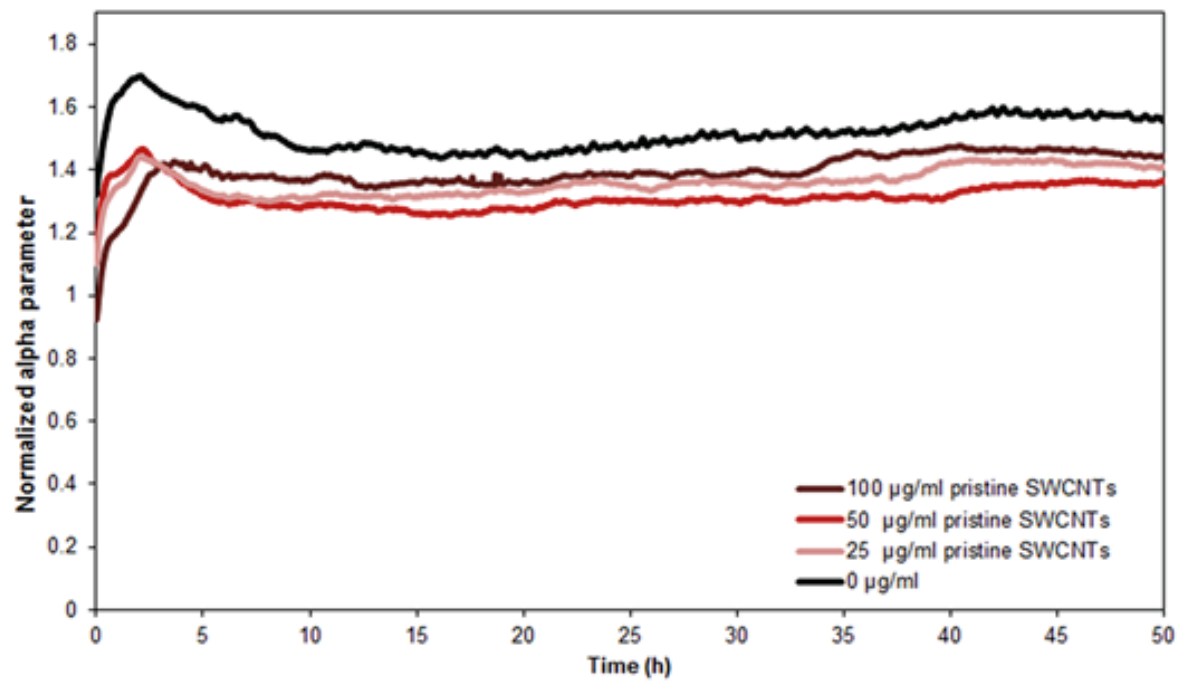

(b)

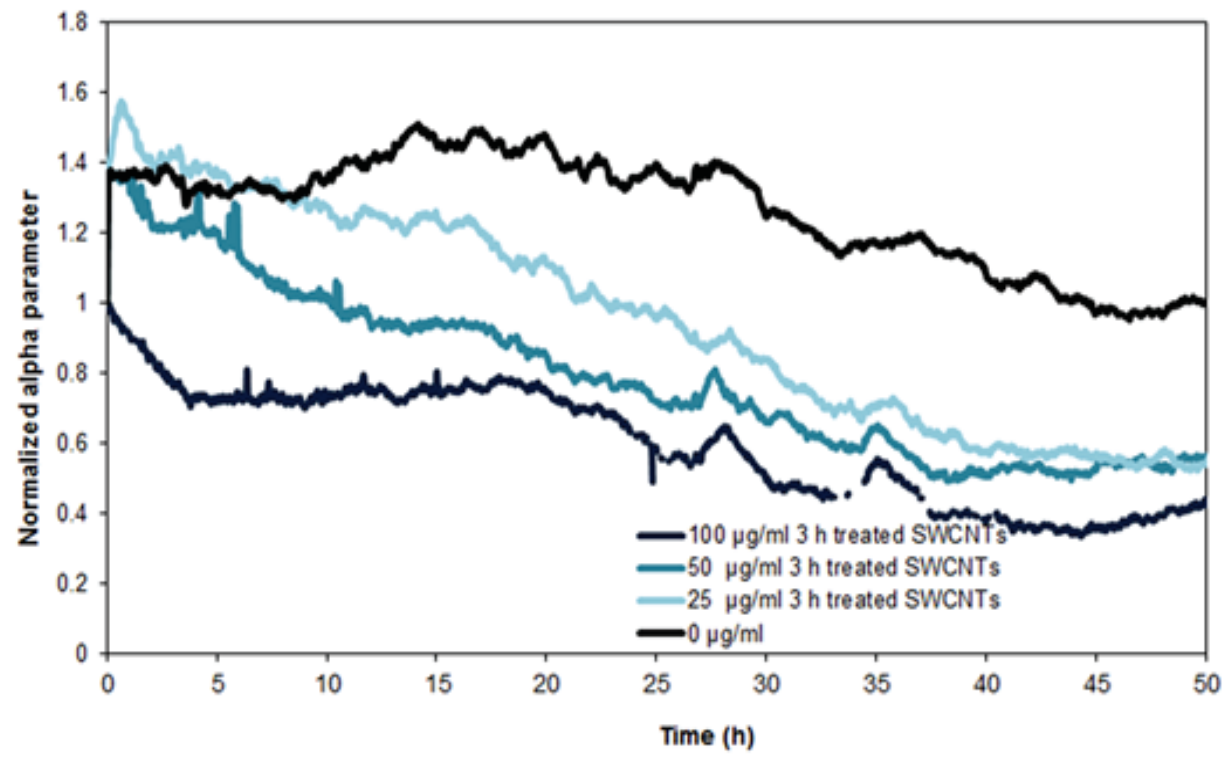


(c)

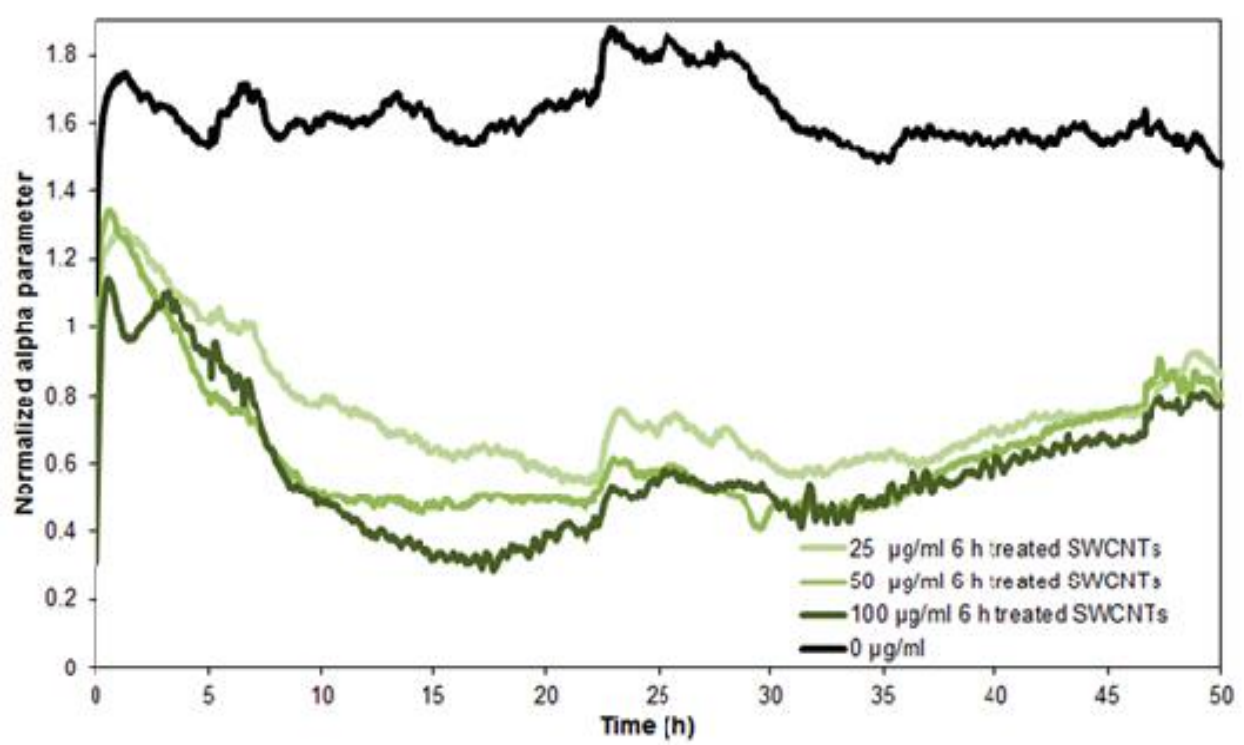

(d)

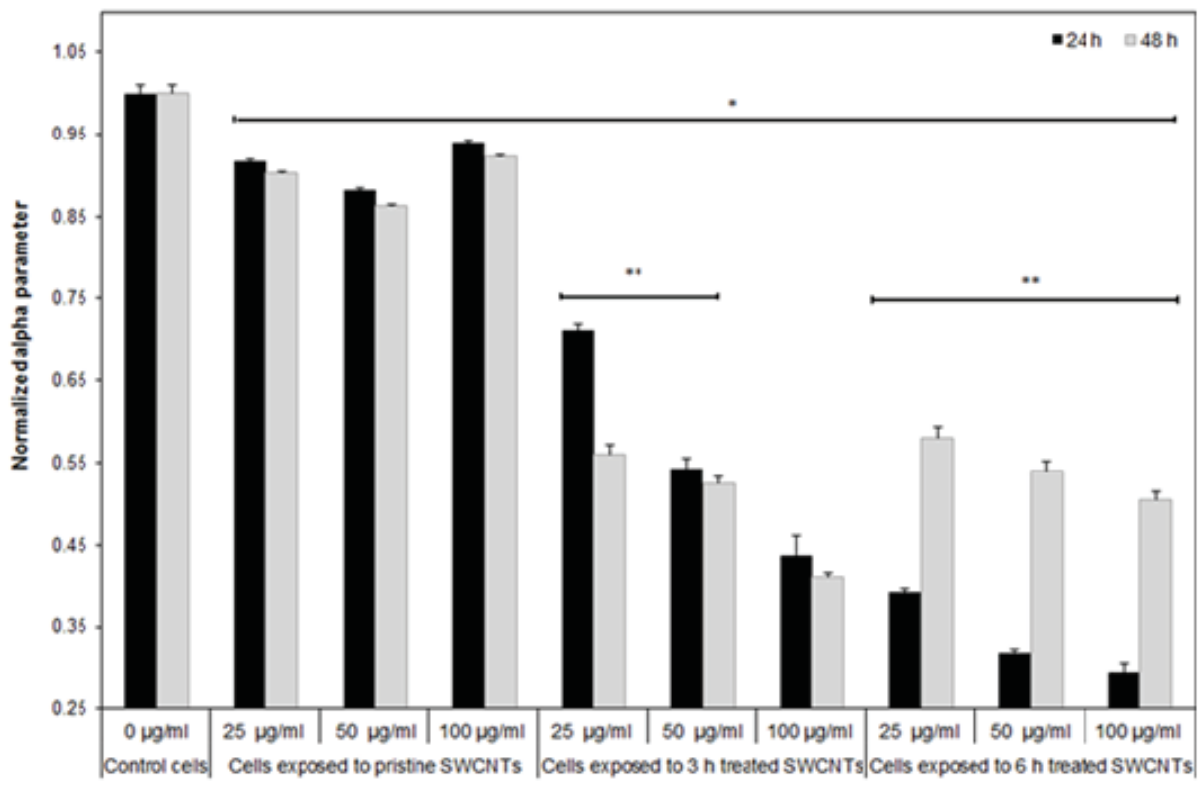

Figure 3: Real-time measurements of the normalized alpha following exposure to a) pristine, b) $3 \mathrm{~h}$ and c) $6 \mathrm{~h}$ acid treated SWCNTs. d) Cross-comparison in alpha parameter following SWCNTs exposure. (* Indicates significant difference between cells exposed to SWCNTs and control cells; ${ }^{* *}$ indicates significant difference between 24 and $48 \mathrm{~h}$ of exposure). 


\section{Cellular viability is function of the SWCNT physico-chemical properties}

To test whether exposure to the SWCNTs library affected cellular viability, we scored the number of cells showing apoptotic percentages based on the changes in their nuclear morphologies as a result of chromatin condensation or DNA fragmentation [47]. Our results indicated that exposure to the SWCNTs induced a time and dose dependent increase in the percentage of apoptotic cells (Figure 4). In particular, cells exposed to pristine SWCNTs showed the highest apoptotic percentage relative to cells exposed to the acid treated SWCNTs with exposure to pristine SWCNTs yielding a maximum of $12 \%$ and $16 \%$ apoptotic cells after 24 and $48 \mathrm{~h}$ respectively. However, exposure to $3 \mathrm{~h}$ acid treated SWCNTs resulted in maximum 5\% apoptotic cells after 24 $\mathrm{h}$ and $11 \%$ after $48 \mathrm{~h}$ exposure respectively, while exposure to $6 \mathrm{~h}$ acid treated SWCNTs resulted in $7 \%$ apoptotic cells after $24 \mathrm{~h}$ and $13 \%$ after $48 \mathrm{~h}$ respectively.

Our analysis evaluating the number of live cells after 24 and $48 \mathrm{~h}$ exposure to the SWCNTs library complemented the results above (Fig. 4b) with examinations showing time, dose and SWCNT-properties dependent decrease in cellular viability of the exposed cells, all relative to controls. For instance, exposure to pristine, 3 and $6 \mathrm{~h}$ treated SWCNTs at 100 and $50 \mu \mathrm{g} / \mathrm{ml}$ doses showed a drop in the percentage of live cells of about $16 \%, 14 \%$ and $10 \%$, with the decrease progressing to $30 \%, 22 \%$ and $18 \%$ respectively after $48 \mathrm{~h}$ of exposure, all relative to controls. Complimentary, $24 \mathrm{~h}$ exposure to $25 \mu \mathrm{g} / \mathrm{ml}$ of pristine, 3 and $6 \mathrm{~h}$ treated SWCNTs did not result in a significant reduction in the live cell counts, however $48 \mathrm{~h}$ exposure reduced the percentage of live cells by about 15\%,12\% and 9\% for the cells exposed to pristine, 3 and $6 \mathrm{~h}$ treated SWCNTs, all relative to control cells. 
(a)

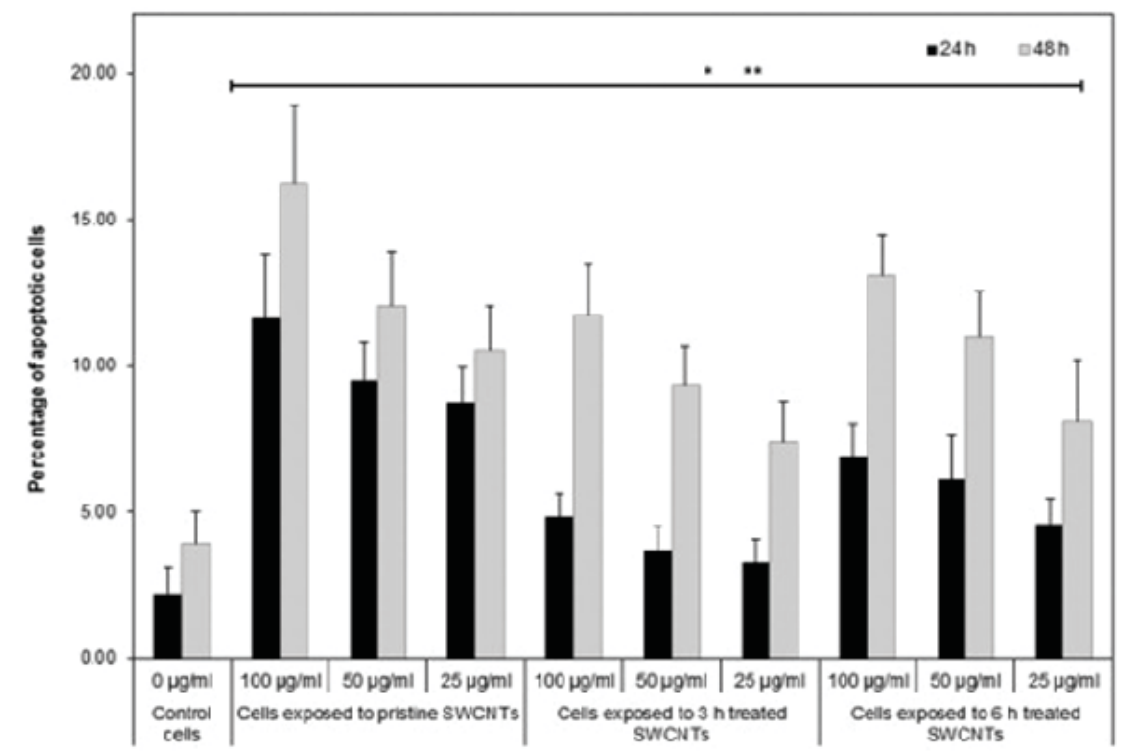

(b)

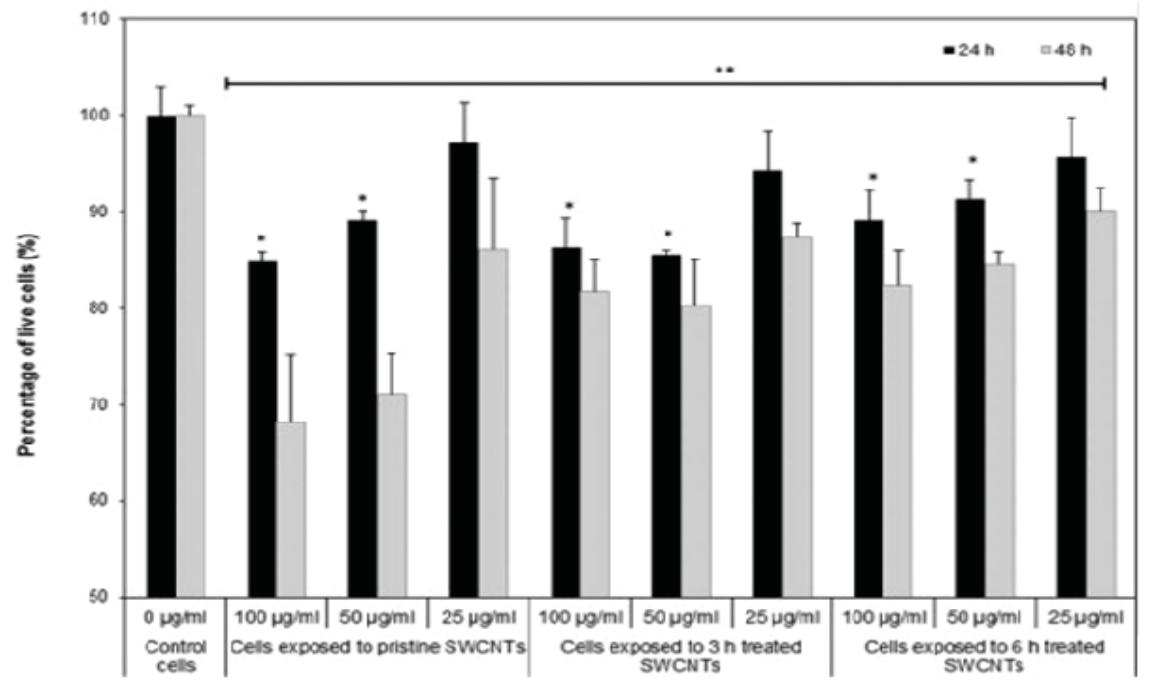

Figure 4: a) Percentage of apoptotic cells and b) percentage of live cells following 24 and $48 \mathrm{~h}$ exposure to different concentrations of pristine, 3 and $6 \mathrm{~h}$ acid treated SWCNTs. (* Indicates significant differences between SWCNTs exposed cells and control cells, $p<0.05$ ). 


\section{Discussion}

Previous studies employing traditional biological methods such as immunofluorescence [34, 48], flow cytometry [49], photospectroscopy [21], and Western blotting [50] have provided insights into the SWCNT-induced toxicity after defined exposure times. Analysis showed that internalization of SWCNTs could activate different cellular mechanisms from ROS generation [18, 32] to inflammation [51], cell signaling [52], protein expression [50], fibrogenesis [53, 54], cellular attachment [55], viability [47] or proliferation [56], with such effects being function of the differences in length [29, 57], agglomeration [31], and surface chemistry [58,59] of the nanotubes being tested. However, such analysis failed to interpret intermediate exposure time points and defined SWCNT-induced toxicity as being the result of invasive cellular changes reflected as cytoskeletal rearrangement [60], membrane disruption [60, 61], disruption of the mitotic spindle [62] or cellular aneuploidy [34, 48], just to name a few.

We provided a comprehensive, multi-parametric, non-invasive, high-throughput and realtime in vitro risk assessment evaluation of the exposure of model human lung epithelial cells to a library of SWCNTs. The library was prepared by incubation of pristine SWCNTs in a strong acid mixture of nitric and sulfuric acid for 3 and 6 h respectively [26, 42]. Raman, EDX and AFM crossanalysis showed that acid treatment increased SWCNTs degree of functionalization with Ocontaining groups by cutting and oxidizing the nanotubes at their defect sites and tips [63] and reduced their content of metal catalysts ( i.e., Fe and Co) incorporated during manufacturing [26]. Analysis also showed that the smaller agglomerate size of the acid treated samples were associated with the formation of thin electric dipole layers that affected SWCNT stability and hydrodynamic diameter [58]. 
The smaller agglomerate size observed for the $3 \mathrm{~h}$ acid treated sample relative to the $6 \mathrm{~h}$ one was further associated with the interplay between the Van der Waals attraction forces and the electrical repulsive forces caused by the O-rich groups according to the Schulze-Hardy rule [64].

Secondly, using the ECIS high-throughput, real-time analysis we showed that changes in cellular behavior upon exposure to SWCNTs were continuously and non-distractively monitored and related to the dose and physico-chemical characteristics (e.g., length, purity, hydrophilicity, and size of agglomerates) of the nanotubes being tested (Figure 5). In particular, the multiple frequencies analysis assessing restrictions on the current pathways through the cellular monolayer as well as through the para- and subcellular spaces [40] helped classify changes in cell morphology as well as in the cell-cell/substrate interactions $[41,44]$ based on the library characteristics. The analysis revealed that the increase in the O-groups led to higher SWCNTs' hydrophilicity and differential internalization [66].

The higher internalization of the $6 \mathrm{~h}$ acid treated SWCNTs relative to both $3 \mathrm{~h}$ acid treated and pristine SWCNTs was reflected as higher changes in both the resistance and cell adhesion properties, presumably due to the higher interactions of these internalized nanotubes with the cellular cytoskeleton and the known role of the cytoskeleton to influence cell-substrate interactions $[60,67]$. This was also confirmed by the ability of the exposed cells to regain some of their adhesion characteristics after $48 \mathrm{~h}$ of exposure nanotube. Further, our results expanded previous studies based on fluorescence microscopy and cell counting techniques by providing quantifiable differential measurements on the cell-cell and cell-substrate interactions without prior cellular manipulation or labeling $[55,68]$. 
Based on previous reports showing that changes in cellular adhesion are associated with changes in cellular morphology [68] and elasticity [49], which in turn could lead to changes in cellular viability $[69,70]$, our analysis of the percentage of apoptotic cells upon exposure to SWCNTs confirmed that the surface chemistry and degree of functionalization of SWCNTs strongly influences cell apoptosis [47, 71-73].

The higher apoptotic percentage observed for cells exposed to pristine SWCNTs was presumably associated with the higher content of metal impurities, as well as the length and larger agglomerate size of these nanotubes relative to their acid treated counterparts. Previous studies showed indeed that exposure to longer SWCNTs induced more potent toxic effects than shorter SWCNTs with exposure to longer nanotubes leading to increased ROS generation [51], fibrosis [74] and apoptosis [51], as well as reduction in cellular viability [57, 74]. Moreover, previous reports have also showed that the presence of metal impurities (i.e., Fe) resulted in higher cytotoxic and genotoxic effects [32], production of hydroxyl radicals [75], loss of intracellular low molecular weight thiols (GSH) and accumulation of lipid hydroperoxides. 


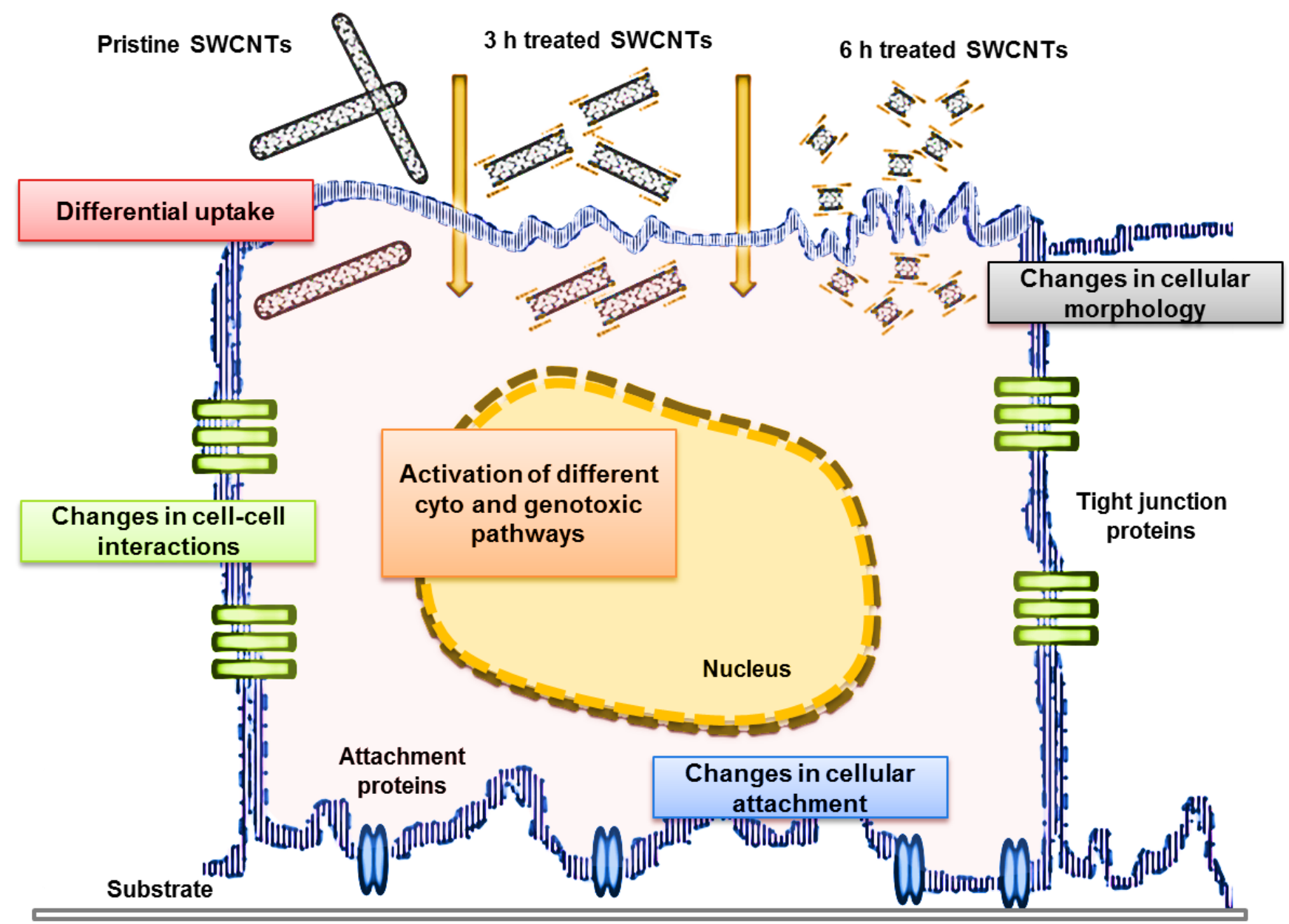

Figure 5: Schematic of the SWCNT-induced cellular changes as a reflection of changes in cellular morphology, cell-cell interactions, and cellular attachment properties. Those changes are analyzed in relation to the SWCNT physico-chemical characteristics to determine nanotubes internalization and induced cellular fate.

Our results confirm previous studies which showed that exposure to longer SWCNTs induced increased toxic effects relative to shorter SWCNTs with exposure to longer nanotubes leading to an increase in the ROS generation and apoptosis, as well as reduction in cellular viability. Further, our results confirm previous reports showing that the presence of metal 
impurities (i.e., $\mathrm{Fe}$ ) in the nanotube samples resulted in higher cytotoxic and genotoxic effects production of hydroxyl radicals, loss of intracellular low molecular weight thiols and accumulation of lipid hydroperoxides (Kagan et al., 2006), all by providing quantifiable differential measurements on the cell-cell and cell-substrate interactions without prior cellular manipulation or labeling.

\section{Conclusions:}

Our study relies on the natural sensitivity of the cells used as primary transducers to provide real-time risk assessment of nanotube toxicity with minimum sample handling thus overcoming the limitations and concerns associated with SWCNT interactions with the biological detection elements. The results showed that exposure of human lung epithelial cells to different doses of SWCNTs with user-defined physical and chemical properties leads to time and dose dependent changes in the cellular behavior, adhesion properties and viability. In particular, our study showed functional differences in the cellular behavior upon exposure to pristine and acid treated SWCNTs according to the length, surface chemistry, purity and agglomeration of the sample being investigated. For instance, while acid treated SWCNTs showed the highest reduction in cellular adhesion, exposure to pristine SWCNTs yielded the highest percentage of apoptotic cells after 24 and $48 \mathrm{~h}$ SWCNT exposure. Our studies help extend the applications of the electric sensing platform to help standardize nanomaterial risk evaluation. 


\section{Acknowledgements:}

This work was supported by National Science Foundation (NSF) grant EPS-1003907 and NSF 1434503, the National Institute of Health (NIH; R01-ES022968). The authors acknowledge use of WVU Shared Research Facilities, the support and expertise of Dr. Karen Martin, the director of the Core Facility, as well as of Dr. Jeremy Hardinger for the initial EDX analysis. 


\section{References:}

1. Ouyang, M., J. L. Huang, and C. M. Lieber, Fundamental electronic properties and applications of single-walled carbon nanotubes. Accounts of Chemical Research, 2002. 35(12): p. 1018-1025.

2. Li, Jinghong and Jin Z. Zhang, Optical properties and applications of hybrid semiconductor nanomaterials. Coordination Chemistry Reviews, 2009. 253(23-24): p. 3015-3041.

3. Baur, Jeff and Edward Silverman, Challenges and Opportunities in Multifunctional Nanocomposite Structures for Aerospace Applications. MRS Bulletin, 2007. 32(04): p. 328-334.

4. Frackowiak, Elzbieta and François Béguin, Carbon materials for the electrochemical storage of energy in capacitors. Carbon, 2001. 39(6): p. 937-950.

5. Merkoçi, Arben, Martin Pumera, Xavier Llopis, Briza Pérez, Manel del Valle, and Salvador Alegret, New materials for electrochemical sensing VI: Carbon nanotubes. TrAC Trends in Analytical Chemistry, 2005. 24(9): p. 826-838.

6. Barone, Paul W., Seunghyun Baik, Daniel A. Heller, and Michael S. Strano, Near-infrared optical sensors based on single-walled carbon nanotubes. Nat Mater, 2005. 4(1): p. 86-92.

7. Bianco, Alberto, Kostas Kostarelos, and Maurizio Prato, Applications of carbon nanotubes in drug delivery. Current Opinion in Chemical Biology, 2005. 9(6): p. 674-679.

8. Golin, Caroline Burkhard, Thomas L. Bougher, Anne Mallow, and Baratunde A. Cola, Toward a comprehensive framework for nanomaterials: An interdisciplinary assessment of the current Environmental Health and Safety Regulation regarding the handling of carbon nanotubes. Journal of Chemical Health and Safety, 2013. 20(4): p. 9-24. 
9. Barillet, S., A. Simon-Deckers, N. Herlin-Boime, M. Mayne-L'Hermite, C. Reynaud, D. Cassio, B. Gouget, and M. Carriere, Toxicological consequences of $\mathrm{TiO} 2, \mathrm{SiC}$ nanoparticles and multi-walled carbon nanotubes exposure in several mammalian cell types: an in vitro study. Journal of Nanoparticle Research, 2010. 12(1): p. 61-73.

10. Clément, Laura, Arnaud Zenerino, Charlotte Hurel, Sonia Amigoni, Elisabeth Taffin de Givenchy, Frédéric Guittard, and Nicolas Marmier, Toxicity assessment of silica nanoparticles, functionalised silica nanoparticles, and HASE-grafted silica nanoparticles. Science of The Total Environment, 2013. 450-451(0): p. 120-128.

11. Speranza, Anna, Rita Crinelli, Valeria Scoccianti, Anna Rita Taddei, Marta Iacobucci, Priyanka Bhattacharya, and $\mathrm{Pu}$ Chun $\mathrm{Ke}$, In vitro toxicity of silver nanoparticles to kiwifruit pollen exhibits peculiar traits beyond the cause of silver ion release. Environmental Pollution, 2013. 179(0): p. 258-267.

12. Gui, S, Z Zhang, L Zheng, Y Cui, X Liu, N Li, X Sang, Q Sun, G Gao, Z Cheng, L Wang, M Tang, and F Hong, Molecular mechanism of kidney injury of mice caused by exposure to titanium dioxide nanoparticles. J Hazard Mater, 2011. 195: p. 365 - 370.

13. Vittori Antisari, Livia, Serena Carbone, Antonietta Gatti, Gilmo Vianello, and Paolo Nannipieri, Toxicity of metal oxide (CeO2, $\mathrm{Fe} 3 \mathrm{O} 4, \mathrm{SnO} 2$ ) engineered nanoparticles on soil microbial biomass and their distribution in soil. Soil Biology and Biochemistry, 2013. 60(0): p. 87-94.

14. Mulchandani, Ashok and Amarjeet S. Bassi, Principles and Applications of Biosensors for Bioprocess Monitoring and Control. Critical Reviews in Biotechnology, 1995. 15(2): p. $105-124$.

15. Zhao, Y., J. L. Lawrie, K. R. Beavers, P. E. Laibinis, and S. M. Weiss, Effect of DNA 
Induced Corrosion on Passivated Porous Silicon Biosensors. ACS Appl Mater Interfaces, 2014.

16. Gliga, AndaR, Sara Skoglund, Inger Odnevall Wallinder, Bengt Fadeel, and HannaL Karlsson, Size-dependent cytotoxicity of silver nanoparticles in human lung cells: the role of cellular uptake, agglomeration and Ag release. Particle and Fibre Toxicology, 2014. 11(1): p. 1-17.

17. Jain, Suneil, Jonathan A. Coulter, Karl T. Butterworth, Alan R. Hounsell, Stephen J. McMahon, Wendy B. Hyland, Mark F. Muir, Glenn R. Dickson, Kevin M. Prise, Fred J. Currell, David G. Hirst, and Joe M. O’Sullivan, Gold nanoparticle cellular uptake, toxicity and radiosensitisation in hypoxic conditions. Radiotherapy and Oncology, 2014. 110(2): p. $342-347$.

18. Jaeger, Alexandra, Dieter G. Weiss, Ludwig Jonas, and Ralf Kriehuber, Oxidative stressinduced cytotoxic and genotoxic effects of nano-sized titanium dioxide particles in human HaCaT keratinocytes. Toxicology, 2012. 296(1-3): p. 27-36.

19. Kostarelos, Kostas, Lara Lacerda, Giorgia Pastorin, Wei Wu, WieckowskiSebastien, Jacqueline Luangsivilay, Sylvie Godefroy, Davide Pantarotto, Jean-Paul Briand, Sylviane Muller, Maurizio Prato, and Alberto Bianco, Cellular uptake of functionalized carbon nanotubes is independent of functional group and cell type. Nat Nano, 2007. 2(2): p. 108113.

20. Nowak, JS, D Mehn, P Nativo, CP Garcia, S Gioria, I Ojea-Jimenez, D Gilliland, and F Rossi, Silica nanoparticle uptake induces survival mechanism in A549 cells by the activation of autophagy but not apoptosis. Toxicol Lett, 2014. 224: p. 84 - 92.

21. Monteiro-Riviere, N. A., A. O. Inman, and L. W. Zhang, Limitations and relative utility of 
screening assays to assess engineered nanoparticle toxicity in a human cell line. Toxicology and Applied Pharmacology, 2009. 234(2): p. 222-235.

22. Dhawan, A. and V. Sharma, Toxicity assessment of nanomaterials: methods and challenges. Anal Bioanal Chem, 2010. 398(2): p. 589-605.

23. Worle-Knirsch, J. M., K. Pulskamp, and H. F. Krug, Oops they did it again! Carbon nanotubes hoax scientists in viability assays. Nano Lett, 2006. 6(6): p. 1261-8.

24. Kroll, Alexandra, Mike H. Pillukat, Daniela Hahn, and Jürgen Schnekenburger, Current in vitro methods in nanoparticle risk assessment: Limitations and challenges. European Journal of Pharmaceutics and Biopharmaceutics, 2009. 72(2): p. 370-377.

25. Marcolongo, G, G Ruaro, M Gobbo, and M Meneghetti, Amino acid functionalization of double-wall carbon nanotubes studied by Raman spectroscopy. Chem Commun (Camb), 2007. 5: p. 4925 - 4927.

26. Dong, C., M. L. Kashon, D. Lowry, J. S. Dordick, S. H. Reynolds, Y. Rojanasakul, L. M. Sargent, and C. Z. Dinu, Exposure to carbon nanotubes leads to changes in the cellular biomechanics. Adv Healthc Mater, 2013. 2(7): p. 945-51.

27. Erdely, A, M Dahm, BT Chen, PC Zeidler-Erdely, JE Fernback, ME Birch, DE Evans, ML Kashon, JA Deddens, and T Hulderman, Carbon nanotube dosimetry: from workplace exposure assessment to inhalation toxicology. Particle and Fibre Toxicology, 2013. 10: p. 53.

28. Lee, JH, SB Lee, GN Bae, KS Jeon, JU Yoon, JH Ji, JH Sung, BG Lee, and JS Yang, Exposure assessment of carbon nanotube manufacturing workplaces. Inhal Toxicol, 2010. 22: p. $369-381$.

29. Sato, Y., A. Yokoyama, K. Shibata, Y. Akimoto, S. Ogino, Y. Nodasaka, T. Kohgo, K. 
Tamura, T. Akasaka, M. Uo, K. Motomiya, B. Jeyadevan, M. Ishiguro, R. Hatakeyama, F. Watari, and K. Tohji, Influence of length on cytotoxicity of multi-walled carbon nanotubes against human acute monocytic leukemia cell line THP-1 in vitro and subcutaneous tissue of rats in vivo. Mol Biosyst, 2005. 1(2): p. 176-82.

30. Nagai, H, Y Okazaki, SH Chew, N Misawa, Y Yamashita, S Akatsuka, T Ishihara, K Yamashita, Y Yoshikawa, and $\mathrm{H}$ Yasui, Diameter and rigidity of multiwalled carbon nanotubes are critical factors in mesothelial injury and carcinogenesis. Proc Natl Acad Sci USA, 2011. 108: p. E1330 - E1338.

31. Wick, Peter, Pius Manser, Ludwig K. Limbach, Ursula Dettlaff-Weglikowska, Frank Krumeich, Siegmar Roth, Wendelin J. Stark, and Arie Bruinink, The degree and kind of agglomeration affect carbon nanotube cytotoxicity. Toxicology Letters, 2007. 168(2): p. 121-131.

32. Aldieri, E., I. Fenoglio, F. Cesano, E. Gazzano, G. Gulino, D. Scarano, A. Attanasio, G. Mazzucco, D. Ghigo, and B. Fubini, The role of iron impurities in the toxic effects exerted by short multiwalled carbon nanotubes (MWCNT) in murine alveolar macrophages. J Toxicol Environ Health A, 2013. 76(18): p. 1056-71.

33. Saxena, Rajiv K., Wanda Williams, John K. McGee, Mary J. Daniels, Elizabeth Boykin, and M. Ian Gilmour, Enhanced in vitro and in vivo toxicity of poly-dispersed acidfunctionalized single-wall carbon nanotubes. Nanotoxicology, 2007. 1(4): p. 291-300.

34. Siegrist, Katelyn, Steven Reynolds, Michael Kashon, David Lowry, Chenbo Dong, Ann Hubbs, Shih-Houng Young, Jeffrey Salisbury, Dale Porter, Stanley Benkovic, Michael McCawley, Michael Keane, John Mastovich, Kristin Bunker, Lorenzo Cena, Mark Sparrow, Jacqueline Sturgeon, Cerasela Dinu, and Linda Sargent, Genotoxicity of multi- 
walled carbon nanotubes at occupationally relevant doses. Particle and Fibre Toxicology, 2014. 11(1): p. 6 .

35. Eldawud, R., T. A. Stueckle, S. Manivannan, H. Elbaz, M. Chen, Y. Rojanasakul, and C. Z. Dinu, Real-time analysis of the effects of toxic, therapeutic and sub-therapeutic concentrations of digitoxin on lung cancer cells. Biosensors and Bioelectronics, 2014. 59: p. 192-199.

36. Opp, Daniel, Brian Wafula, Jennifer Lim, Eric Huang, Jun-Chih Lo, and Chun-Min Lo, Use of electric cell-substrate impedance sensing to assess in vitro cytotoxicity. Biosensors and Bioelectronics, 2009. 24(8): p. 2625-2629.

37. Xiao, C., B. Lachance, G. Sunahara, and J. H. Luong, Assessment of cytotoxicity using electric cell-substrate impedance sensing: concentration and time response function approach. Anal Chem, 2002. 74(22): p. 5748-53.

38. Campbell, Alan S., Chenbo Dong, Jonathan S. Dordick, and Cerasela Zoica Dinu, BioNano engineered hybrids for hypochlorous acid generation. Process Biochemistry, 2013. 48(9): p. $1355-1360$.

39. Marshall, M. W., S. Popa-Nita, and J. G. Shapter, Measurement of functionalised carbon nanotube carboxylic acid groups using a simple chemical process. Carbon, 2006. 44(7): p. $1137-1141$.

40. Tiruppathi, C., A. B. Malik, P. J. Del Vecchio, C. R. Keese, and I. Giaever, Electrical method for detection of endothelial cell shape change in real time: assessment of endothelial barrier function. Proc Natl Acad Sci U S A, 1992. 89(17): p. 7919-23.

41. Giaever, Ivar and Charles R. Keese, A morphological biosensor for mammalian cells. Nature, 1993. 366(6455): p. 591-592. 
42. Wepasnick, Kevin A., Billy A. Smith, Kaitlin E. Schrote, Hannah K. Wilson, Stephen R. Diegelmann, and D. Howard Fairbrother, Surface and structural characterization of multiwalled carbon nanotubes following different oxidative treatments. Carbon, 2011. 49(1): $\mathrm{p}$. 24-36.

43. Liu, J., A. G. Rinzler, H. J. Dai, J. H. Hafner, R. K. Bradley, P. J. Boul, A. Lu, T. Iverson, K. Shelimov, C. B. Huffman, F. Rodriguez-Macias, Y. S. Shon, T. R. Lee, D. T. Colbert, and R. E. Smalley, Fullerene pipes. Science, 1998. 280(5367): p. 1253-1256.

44. Keese, Charles R., ECIS-based High Throughput Screening Instrumentation, 2005, NATIONAL CENTER FOR RESEARCH RESOURCES.

45. Luanpitpong, Sudjit, Liying Wang, Vincent Castranova, and Yon Rojanasakul, Induction of stem-like cells with malignant properties by chronic exposure of human lung epithelial cells to single-walled carbon nanotubes. Particle and Fibre Toxicology, 2014. 11(1): p. 22.

46. Sargent, LM, SH Reynolds, D Lowry, ML Kashon, SA Benkovic, JL Salisbury, AF Hubbs, MJ Keane, J Mastovich, and KL Bunker, Genotoxicity of multi-walled carbon nanotubes at occupationally relevant doses. Proc Am Assoc Cancer Res, 2012. 53: p. 1320.

47. Cui, Daxiang, Furong Tian, Cengiz S. Ozkan, Mao Wang, and Huajian Gao, Effect of single wall carbon nanotubes on human HEK293 cells. Toxicology Letters, 2005. 155(1): p. 7385.

48. Sargent, L. M., A. A. Shvedova, A. F. Hubbs, J. L. Salisbury, S. A. Benkovic, M. L. Kashon, D. T. Lowry, A. R. Murray, E. R. Kisin, S. Friend, K. T. McKinstry, L. Battelli, and S. H. Reynolds, Induction of Aneuploidy by Single-Walled Carbon Nanotubes. Environ Mol Mutagen, 2009. 50(8): p. 708-717.

49. Dong, C., R. Eldawud, L. M. Sargent, M. L. Kashon, D. Lowry, Y. Rojanasakul, and C. Z. 
Dinu, Towards Elucidating the Effects of Purified MWCNTs on Human Lung Epithelial cells. Environ Sci Nano, 2014. 1(6): p. 95-603.

50. Lai, Xianyin, Bonnie L. Blazer-Yost, James W. Clack, Sharry L. Fears, Somenath Mitra, Susana Addo Ntim, Heather N. Ringham, and Frank A. Witzmann, Protein expression profiles of intestinal epithelial co-cultures: effect of functionalised carbon nanotube exposure. International journal of biomedical nanoscience and nanotechnology, 2013. 3(12): p. 10.1504/IJBNN.2013.054508.

51. van Berlo, D., V. Wilhelmi, A. W. Boots, M. Hullmann, T. A. Kuhlbusch, A. Bast, R. P. Schins, and C. Albrecht, Apoptotic, inflammatory, and fibrogenic effects of two different types of multi-walled carbon nanotubes in mouse lung. Arch Toxicol, 2014.

52. Ellinger-Ziegelbauer, Heidrun and Jürgen Pauluhn, Pulmonary toxicity of multi-walled carbon nanotubes (Baytubes $\AA$ ) relative to $\alpha$-quartz following a single $6 \& \# x a 0 ;$; inhalation exposure of rats and a 3 months post-exposure period. Toxicology, 2009. 266(1-3): p. 1629.

53. Porter, D. W., A. F. Hubbs, R. R. Mercer, N. Q. Wu, M. G. Wolfarth, K. Sriram, S. Leonard, L. Battelli, D. Schwegler-Berry, S. Friend, M. Andrew, B. T. Chen, S. Tsuruoka, M. Endo, and V. Castranova, Mouse pulmonary dose- and time course-responses induced by exposure to multi-walled carbon nanotubes. Toxicology, 2010. 269(2-3): p. 136-147.

54. Muller, Julie, François Huaux, Nicolas Moreau, Pierre Misson, Jean-François Heilier, Monique Delos, Mohammed Arras, Antonio Fonseca, Janos B. Nagy, and Dominique Lison, Respiratory toxicity of multi-wall carbon nanotubes. Toxicology and Applied Pharmacology, 2005. 207(3): p. 221-231.

55. Kaiser, J. P., T. Buerki-Thurnherr, and P. Wick, Influence of single walled carbon nanotubes 
at subtoxical concentrations on cell adhesion and other cell parameters of human epithelial cells. Journal of King Saud University - Science, 2013. 25(1): p. 15-27.

56. Müller, Jakob, Christian Thirion, and Michael W. Pfaffl, Electric cell-substrate impedance sensing (ECIS) based real-time measurement of titer dependent cytotoxicity induced by adenoviral vectors in an IPI-2I cell culture model. Biosensors and Bioelectronics, 2011. 26(5): p. 2000-2005.

57. Manke, Amruta, Sudjit Luanpitpong, Chenbo Dong, Liying Wang, Xiaoqing He, Lori Battelli, Raymond Derk, Todd Stueckle, Dale Porter, Tina Sager, Honglei Gou, Cerasela Dinu, Nianqiang Wu, Robert Mercer, and Yon Rojanasakul, Effect of Fiber Length on Carbon Nanotube-Induced Fibrogenesis. International Journal of Molecular Sciences, 2014. 15(5): p. 7444-7461.

58. Jiang, Jingkun, Günter Oberdörster, and Pratim Biswas, Characterization of size, surface charge, and agglomeration state of nanoparticle dispersions for toxicological studies. Journal of Nanoparticle Research, 2009. 11(1): p. 77-89.

59. Liu, Z. B., X. Dong, L. P. Song, H. L. Zhang, L. X. Liu, D. W. Zhu, C. X. Song, and X. G. Leng, Carboxylation of multiwalled carbon nanotube enhanced its biocompatibility with L02 cells through decreased activation of mitochondrial apoptotic pathway. Journal of Biomedical Materials Research Part A, 2014. 102(3): p. 665-673.

60. Umemoto, E. Y., M. Speck, L. M. Shimoda, K. Kahue, C. Sung, A. J. Stokes, and H. Turner, Single-walled carbon nanotube exposure induces membrane rearrangement and suppression of receptor-mediated signalling pathways in model mast cells. Toxicol Lett, 2014. 229(1): p. 198-209.

61. Chang, Rakwoo and Angela Violi, Insights into the Effect of Combustion-Generated 
Carbon Nanoparticles on Biological Membranes: A Computer Simulation Study. The Journal of Physical Chemistry B, 2006. 110(10): p. 5073-5083.

62. Sargent, L. M., A. F. Hubbs, S. H. Young, M. L. Kashon, C. Z. Dinu, J. L. Salisbury, S. A. Benkovic, D. T. Lowry, A. R. Murray, E. R. Kisin, K. J. Siegrist, L. Battelli, J. Mastovich, J. L. Sturgeon, K. L. Bunker, A. A. Shvedova, and S. H. Reynolds, Single-walled carbon nanotube-induced mitotic disruption. Mutat Res, 2012. 745(1-2): p. 28-37.

63. Hu, Hui, Bin Zhao, Mikhail E. Itkis, and Robert C. Haddon, Nitric Acid Purification of Single-Walled Carbon Nanotubes. The Journal of Physical Chemistry B, 2003. 107(50): p. 13838-13842.

64. Desai, Chintal, Kun Chen, and Somenath Mitra, Aggregation behavior of nanodiamonds and their functionalized analogs in an aqueous environment. Environmental Science: Processes \& Impacts, 2014. 16(3): p. 518-523.

65. Sano, Masahito, Junko Okamura, and Seiji Shinkai, Colloidal Nature of Single-Walled Carbon Nanotubes in Electrolyte Solution: The Schulze-Hardy Rule. Langmuir, 2001. 17(22): p. 7172-7173.

66. Mu, Qingxin, Dana L. Broughton, and Bing Yan, Endosomal Leakage and Nuclear Translocation of Multiwalled Carbon Nanotubes: Developing a Model for Cell Uptake. Nano Lett, 2009. 9(12): p. 4370-4375.

67. Holt, B. D., P. A. Short, A. D. Rape, Y. L. Wang, M. F. Islam, and K. N. Dahl, Carbon Nanotubes Reorganize Actin Structures in Cells and ex Vivo. Acs Nano, 2010. 4(8): p. $4872-4878$.

68. Song, Maoyong, Luzhe Zeng, Shaopeng Yuan, Junfa Yin, Hailin Wang, and Guibin Jiang, Study of cytotoxic effects of single-walled carbon nanotubes functionalized with different 
chemical groups on human MCF7 cells. Chemosphere, 2013. 92(5): p. 576-582.

69. Cross, S. E., Y. S. Jin, J. Rao, and J. K. Gimzewski, Nanomechanical analysis of cells from cancer patients. Nature Nanotechnology, 2007. 2(12): p. 780-783.

70. Snyder-Talkington, B. N., D. Schwegler-Berry, V. Castranova, Y. Qian, and N. L. Guo, Multi-walled carbon nanotubes induce human microvascular endothelial cellular effects in an alveolar-capillary co-culture with small airway epithelial cells. Particle and Fibre Toxicology, 2013. 10.

71. Patlolla, Anita, Brionna Knighten, and Paul Tchounwou, Multi-Walled Carbon Nanotubes Induce Cytotoxicity, Genotoxicity And Apoptosis In Normal Human Dermal Fibroblast Cells. Ethnicity \& disease, 2010. 20(1 Suppl 1): p. S1-65-72.

72. Ye, Shefang, Yuanqin Jiang, Honggang Zhang, Yifang Wang, Yihui Wu, Zhenqing Hou, and Qiqing Zhang, Multi-Walled Carbon Nanotubes Induce Apoptosis in RAW 264.7 CellDerived Osteoclasts Through Mitochondria-Mediated Death Pathway. Journal of Nanoscience and Nanotechnology, 2012. 12(3): p. 2101-2112.

73. Dong, C. B., A. S. Campell, R. Eldawud, G. Perhinschi, Y. Rojanasakul, and C. Z. Dinu, Effects of acid treatment on structure, properties and biocompatibility of carbon nanotubes. Applied Surface Science, 2013. 264: p. 261-268.

74. Luanpitpong, Sudjit, Liying Wang, Amruta Manke, Karen H. Martin, Amanda Gatesman Ammer, Vincent Castranova, Yong Yang, and Yon Rojansakul, Induction of Stemlike Cells with Fibrogenic Properties by Carbon Nanotubes and Its Role in Fibrogenesis. Nano Lett, 2014. 14(6): p. 3110-3116.

75. Kagan, V. E., Y. Y. Tyurina, V. A. Tyurin, N. V. Konduru, A. I. Potapovich, A. N. Osipov, E. R. Kisin, D. Schwegler-Berry, R. Mercer, V. Castranova, and A. A. Shvedova, Direct 
and indirect effects of single walled carbon nanotubes on RAW 264.7 macrophages: Role of iron. Toxicology Letters, 2006. 165(1): p. 88-100. 


\title{
Chapter 2
}

\section{Combinatorial Approaches to Evaluate Nanodiamonds Uptake and Induced Cellular Fate}

\begin{abstract}
Nanodiamonds (NDs) are an emerging class of engineered nanomaterials that hold great promise for the next generation of bionanotechnological products to be used for drug and gene delivery, or for bio-imaging and biosensing. Previous studies have shown that upon their cellular uptake, NDs exhibit high biocompatibility in various in vitro and in vivo set-ups. Herein we hypothesized that the increased NDs biocompatibility is a result of minimum membrane perturbations and their reduced ability to induce disruption or damage during cellular translocation. Using multi-scale combinatorial approaches that simulate ND-membrane interactions, we correlated NDs real-time cellular uptake and kinetics with the ND-induced membrane fluctuations to derive energy requirements for the uptake to occur. Our discrete and real-time analyses showed that the majority of NDs internalization occurs within $2 \mathrm{~h}$ of cellular exposure, however, with no effects on cellular viability, proliferation or cellular behavior. Furthermore, our simulation analyses using coarse-grained models identified key changes in the energy profile, membrane deformation and recovery time, all function of the average ND or ND-based agglomerate size. Understanding the mechanisms responsible for ND-cell membrane interactions could possibly advance their implementation in various biomedical applications.
\end{abstract}




\section{Introduction}

Nanodiamonds (NDs) are an emerging class of engineered carbon-based nanomaterials known for their narrow size distribution and high surface area, light weight, high adsorption capability, chemical and thermal stability, as well as biocompatibility [1,2]. Their ability to allow multi-surface functionalization by bio-conjugation with nucleic acids, proteins and therapeutic agents[3, 4] make NDs viable candidates for a variety of applications in the biomedical, pharmaceutical, and industrial fields[5]. In particular, the inexpensive and large-scale production of NDs through detonation of carbon-containing explosives could lead to the formation of the next generation of smart systems to be used as drug delivery platforms in cancer treatment, as carriers of genetic material and proteins, or as bio-imaging probes[6]. However, before such large-scale implementation could be achieved, NDs uptake, internalization, interactions and ultimately induced cellular fate need to be fully assessed.

Previous studies have showed that NDs have minimum toxicity in different cellular systems; for instance, Liu et al., showed that exposure of human lung epithelial cells A549 and HFL-1 normal fibroblasts to ND concentrations between $0.1-100 \mu \mathrm{g} / \mathrm{ml}$ did not affect cellular viability nor altered protein expression profiles[7]. Complementarily, studies of cellular morphological changes and DNA fragmentation in macrophages and HT-29 human colorectal adenocarcinoma cells respectively did not show cell transformation[8] nor significant reactive oxygen species generation[7, 9]. Additionally, Schrand et al., showed that toxicity of NDs was significantly lower when compared to the toxicity of other carbon-based nanomaterials, with the order of toxicity decreasing from single-walled (SW) to multi-walled carbon nanotubes (MWCNTs), to carbon black (CB) and NDs[9] respectively. Furthermore, in vivo investigations using animal models complemented in vitro analyses, with histopathological and ultrastructural 
images of biological tissues showing that NDs did not induce pulmonary toxicity when administered to mice by intratracheal instillation[8]. Similarly, subcutaneous exposure, intraperitoneal injection or oral administration for different periods (from 10 days up to 3 months) in both mice and rats did not lead to inflammatory or adverse immune reactions[10] nor weight changes of mice and rats, nor of their offsprings internal organ (e.g., lungs, kidneys, and heart)[8, 11]. While the majority of studies reported high biocompatibility of NDs in vivo, however, some concentration and functionalities of the NDs were shown to induce thromboembolism in mice[12].

Although these results show that the toxicity of NDs has been extensively investigated, there is limited available information that revealed NDs dynamics upon cellular uptake or translocation. For instance, previous research indicated that NDs are uptaken through an energydependent clathrin-mediated endocytotic pathway[13, 14] at a higher cellular uptake rate than the rate of other carbon-based nanomaterials (i.e., SW-, MWCNTs or graphene for instance)[15] however, such studies failed to demonstrate why the proposed pathway was the preferred mechanism. Given that the real-time uptake and internalization of NDs rely on the average particle size as well as on ND interaction with the proteins in cellular media[16, 17], understanding the mechanisms responsible for ND-cell membrane interactions as well as possible ND-induced membrane damage will provide fundamental knowledge to help explain their observed biocompatibility and thus ensure further implementation as smart drug delivery nanovehicles[16].

Herein, we hypothesize that the increased biocompatibility of NDs is due to minimum membrane damage induced by their uptake. Using combinatorial platforms that simulate ND interactions with the cellular membrane and cells as primary transducers, we unravel NDs realtime uptake, translocation, as well as induced-cellular fate in model cellular systems[18] to provide novel scientific insights that help advance their implementation in biomedical applications. 


\section{Materials and Methods}

\section{Nanodiamonds (NDs) preparation}

Detonation nanodiamonds powder (NDs) was purchased from Plasmachem (GmbH, Germany) and grinded using a planetary ball mill (Fritsch Pulverisette 7 premium line, Germany) and zirconia oxide balls (100 $\mu \mathrm{m}$ of diameter, Germany) at $7000 \mathrm{U} / \mathrm{min}$, two times for $30 \mathrm{~min}$ each, with a 10 min break in between. The resulting grinded powder was suspended in Milli-Q water to obtain a $1 \mathrm{wt} \%$ solution; subsequently, the solution was sonicated in pulsed mode in an ultrasonic homogenizer (Bandelin Sonoplus HD 2200; control unit GM 2200, Germany) at a cycle rate of $50 \%$ and the $\mathrm{pH}$ was adjusted to 7.4 .

\section{Chemical and physical characterization of NDs}

Fourier transform infrared spectroscopy (FTIR) was performed on a Smart OMNITransmission module (Thermo Scientific, Nicolet IZ10, Germany); at least 32 scans per sample in the range of $400-4000 \mathrm{~cm}^{-1}$ and at a resolution of $4 \mathrm{~cm}^{-1}$ were acquired and analyzed using the manufacturer software (Omnic, Germany). The size and morphology of the NDs were evaluated using scanning (SEM) and transmission electron (TEM) microscopy. SEM investigations were performed on a Carl Zeiss 1.500stes Gemini SEM (GmbH, Germany); for this, a droplet of a $0.1 \mathrm{wt} \%$ NDs suspension was dispersed in water and dried on a silicon wafer. TEM images were obtained on a Carl Zeiss LIBRA ${ }^{\circledR} 200(\mathrm{GmbH}$, Germany) by placing small amounts of a 1 wt\% NDs suspension on a clean copper-grid with a carbon-film with holes (400 mesh, Plano Germany). Analysis of NDs aggregation and zeta potential were performed using dynamic light scattering (Delsa ${ }^{\mathrm{TM}}$ Nano Particle Analyzer Beckman Coulter, Germany). In each experiment, suspensions of NDs $(100 \mu \mathrm{g} / \mathrm{ml})$ were prepared in Milli-Q water or Dulbecco's Modified Eagle Medium 
(DMEM, Invitrogen, USA) supplemented with 5\% Fetal Bovine Serum (FBS, Invitrogen, USA). For each analysis, 4 replicates were performed for a total of 150 measurements, at $20{ }^{\circ} \mathrm{C}$ and considering a 1.33 refractive index for water and a 1.34 for DMEM respectively[19].

\section{Cell culture}

Immortalized human bronchial epithelial cells (BEAS-2B) were purchased from American Type Culture Collection (ATCC, USA) and cultured in DMEM media with 5\% FBS, $0.1 \%$ L-glutamine and $1 \%$ penicillin/streptomycin (Invitrogen, USA). Cells were passaged regularly using $0.25 \%$ trypsin (Invitrogen, USA) and maintained in a humidified atmosphere at $37{ }^{\circ} \mathrm{C}$ and $5 \% \mathrm{CO}_{2}$ respectively.

\section{Uptake of NDs}

BEAS-2B cells were seeded overnight in a 12 -well plate (Fisher, USA) at a density of $2.5 \mathrm{x}$ $10^{5} \mathrm{cell} / \mathrm{ml}$. The cells were subsequently treated with $400,200,100,50$, and $25 \mu \mathrm{g} / \mathrm{ml} \mathrm{NDs}$ suspensions in DMEM media with 5\% FBS for 1, 2, 3 or $24 \mathrm{~h}$ respectively. Following the NDs treatment, the cells were washed with phosphate buffer saline (PBS, Lonza, USA), trypsinized, resuspended in DMEM, centrifuged at room temperature and $1200 \mathrm{rpm}$ for $5 \mathrm{~min}$. The samples were further washed with PBS, fixed with $4 \%$ paraformaldehyde (Fisher Scientific, USA) for 15 min at room temperature, and then washed again with PBS several times to remove any of the fixative.

Fluorescence Activated Cell Sorting (FACS) was performed on a BD LSR Fortessa Flow cell analyzer (BD Biosciences, USA); the forward scatter (FSC) and side scatter (SSC) were used to gate the live cells population and at least 10000 events were recorded for each sample. Data was 
analyzed and plotted using FlowJo v7.2.5 software and NDs uptake was determined by averaging the FITC signal of the exposed cells at $488 \mathrm{~nm}$ excitation and $515 \mathrm{~nm}$ emission.

\section{Electric Cell Impedance Sensing (ECIS)}

Real-time analysis of the BEAS-2B cellular behavior before and after exposure to NDs was performed using an electric cell impedance sensing (ECIS 96W, Applied Biophysics) platform. In each experiment, 96W10+ array (Applied Biophysics, USA) with 20 gold electrodes in interdigited finger configuration was employed to provide concomitant measurements of 2000-4000 cells per well in real-time and at multiple frequencies. For this, the array holder was placed in a humidified incubator at $37{ }^{\circ} \mathrm{C}$ and $5 \% \mathrm{CO}_{2}$ to ensure optimal conditions for cell growth. Prior to any experiment, the electrodes were stabilized for $3 \mathrm{~h}$ in $200 \mu \mathrm{L}$ serum free DMEM; subsequently, BEAS-2B cells were added at a density of $2.5 \times 10^{5}$ cell $/ \mathrm{mL}$ in a volume of $200 \mu \mathrm{L} /$ well. Cells were allowed to settle and grow over the electrodes until a confluent monolayer was formed[20]. Upon monolayer formation, the cells were treated with 400, 200, 100, 50, and $25 \mu \mathrm{g} / \mathrm{ml}$ NDs suspended in DMEM media with 5\% FBS and their cellular behavior was subsequently monitored for $48 \mathrm{~h}$. 


\section{Cell viability}

To determine the effect of NDs on cellular viability, BEAS-2B cells were seeded overnight in a 96-well plate (Fisher, USA) at a density of $1 \times 10^{5}$ cell $/ \mathrm{ml}$ and treated with $400,200,100,50$, and $25 \mu \mathrm{g} / \mathrm{ml} \mathrm{ND}$ suspensions for $24 \mathrm{~h}$. Subsequently, $10 \mu \mathrm{l}$ of tetrazolium salt 2- (4-iodophenyl) - 3- (4-nitrophenyl) - 5-(2,4-disulfophenyl) -2H-tetrazolium, known as WST-1 (Roche, USA), was added to each well and incubated for $2 \mathrm{~h}$. Changes in cell viability were based on the cellular ability to cleave WST-1 salt to farmazan by enzyme dehydrogenase[21]. The amount of farmazan dye formed was assessed using a BioTek 96 plate reader (BioTek, USA) and measuring the absorbance of the sample at $450 \mathrm{~nm}$. Relative cellular viability was calculated as the ratio of absorbance signal as derived from the NDs exposed cells to the absorbance signal of control (i.e., untreated cells).

\section{Cell cycle analysis}

To study the effects of different NDs doses on cell cycle progression, BEAS-2B cells were seeded overnight in 12 well plate at a density of $2.5 \times 10^{5}$ cells/well, and exposed to 400, 200, 100, 50 and $25 \mu \mathrm{g} / \mathrm{ml}$ NDs suspended in DMEM media with 5\% FBS for $24 \mathrm{~h}$. Subsequently, the cells were trypsinized, collected, washed twice with PBS, centrifuged at $1500 \mathrm{rpm}$ for $6 \mathrm{~min}$ and fixed overnight at $-20{ }^{\circ} \mathrm{C}$ in $2 \mathrm{ml} 70 \%$ ethanol (Sigma, USA). Upon time elapsed, the cells were again washed in PBS, resuspended in $0.2 \%$ tween 20 (Sigma, USA) for 15 min, treated with $10 \mu 10.05 \%$ RNase (Sigma, USA) for $15 \mathrm{~min}$ and lastly stained with $30 \mu$ propidium iodide (Sigma, USA). The DNA content was determined using BD LSR Fortessa Flow cell analyzer. Forward scatter (FSC) and side scatter (SSC) were used for gating to include the majority of live cell population; 20,000 events were collected for each sample using the BD FACS Diva software (Verity Software 
House, USA) and analyzed using FlowJo software V10.0.7 (Tree Star Inc., USA) to measure their DNA content.

\section{Simulation setup}

To evaluate the uptake of NDs, we used coarse-grained representation and the GROMACS software package (v4.5.5)[22]. The coarse-grained representation was developed using the MARTINI four-to-one mapping scheme[23]. Atomistic models of NDs $(3,5$, and $7 \mathrm{~nm}$ in diameter) were generated using the diamond lattice (with Fd3m space group and face-centered cubic lattice) in CrystalMaker software. Coarse grained ND beads were given standard C1 bead type and a zero net surface charge. Subsequently, the constructed ND was energy minimized and equilibrated with explicit MARTINI water (isobaric-isothermal ensemble at $300 \mathrm{~K}$ and $1 \mathrm{~atm}$ pressure).

The coarse-grained lipid bilayer used for the analysis was made up of neutral distearoylphosphatidyl choline (DSPC) molecules and negatively charged distearoylphosphatidyl glycerol (DSPG) lipid molecules. The DSPC:DSPG ratio was maintained at a 3:1 ratio to resemble a net negative charge of mammalian cell membranes[24]. The electroneutrality of the total system was achieved by adding the required number of sodium counterions as determined by the number of DSPG lipid molecules being available. The bilayer was allowed to self-assemble without any constraints in a user-designed simulation box with a size of $16.0 \times 16.0 \times 30 \mathrm{~nm}^{3}$. In all simulations the center-of-mass $(\mathrm{COM})$ of the ND was placed $7.0 \mathrm{~nm}$ along the $+z$-axis above the lipid bilayer as the starting structure for the simulation; the entire simulation box was solvated with coarsegrained water beads of which $10 \%$ were anti-freeze type. 
All initial systems were energy minimized using the steepest descent algorithm followed by isochoric-isothermal (NVT) and isobaric-isothermal (NPT) equilibration simulations for $100 \mathrm{~ns}$ each at $300 \mathrm{~K}$. The production run simulations were performed using the Berendsen barostat[25] and temperature coupling at $300 \mathrm{~K}$. The equilibrated systems were subsequently subjected to umbrella sampling pull simulations for $3.6 \mathrm{~ns}$ and over a distance of approximately $13 \mathrm{~nm}$ by applying a constant force of $1000 \mathrm{~kJ} \mathrm{~mol}^{-1} \mathrm{~nm}^{-2}$ to the COM of the ND to pull it through the lipid bilayer. In each case, the geometries of the system were saved at regular time intervals to generate over 174 configuration windows with each window being analyzed to determine the distance between the COM of the ND and the mid-plane of the lipid bilayer. Out of the initial set of configurations, uniformly separated windows with $0.2 \mathrm{~nm}$ spacing were selected. For each of the selected windows, NPT equilibration was performed for $5 \mathrm{~ns}$ followed by $100 \mathrm{~ns}$ of production run with no geometric constraints. At the end of the production run the potential of mean force (PMF) values were obtained for the translocation process by using the weighted histogram analysis method[26]. The activation barrier for each translocation simulation was calculated by taking a difference between the maximum and the minimum energy points of the PMF curve. The rate constants were calculated by using the first order transition state theory; subsequently, the rate constant was used to calculate the half-lives and characteristic lifetime of the translocation process[17].

To mimic the experimental serum protein in medium, bovine serum albumin (PDB: 4F5S) protein[27] was used in the simulations. High resolution BSA crystal structure was obtained from the protein data bank and coarse-grained using the available MARTINI scripts. The secondary structure of the protein and the bead assignment were based on standard MARTINI coarse grained methods[23]. The interactions between coarse grained BSA and NDs of sizes 3, 5, and $7 \mathrm{~nm}$ were 
investigated in three separate simulations. In each case, the NDs were introduced in a wellequilibrated system of BSA molecules with explicit water and positively charged counter ions $\left(\mathrm{Na}^{+}\right)$at $300 \mathrm{~K}$. The counter ions were added to maintain the electroneutrality of the simulation system because BSA is a negatively charged protein under the physiological conditions.

\section{Statistical analysis}

All results are presented as mean \pm standard deviation. Uptake, cellular viability and cell cycle experiments were performed in duplicates and repeated at least 3 times, for a total minimum of 6 replicates per each concentration and each time point being investigated. ECIS experiments were performed in duplicates and repeated at least 4 times per each dose being tested. Two-way analysis of variance (ANOVA) and unpaired two-tailed Student's T-test were performed using SigmaPlot 10.0 (Systat Software Inc.); differences were considered significant for $\mathrm{p}^{*}<0.05$. Each coarse-grained simulation was repeated 2 times to sample over the starting configurations and the initial conditions of the system. The random number generator was initialized with different seeds in each simulation. The statistical errors in the PMF curves were estimated using the bootstrap analysis available in GROMACS. The size analysis of equilibrated ND particle after BSA corona formation was performed by averaging over a multiple inter-particle distances of farthest, diametrically opposite coarse-grained beads of BSA proteins. The average values of the diameter and standard deviation are reported. 


\section{Results}

\section{Physico-chemical characterization of nanodiamonds (NDs)}

Comprehensive analyses of NDs physico-chemical properties were performed using infrared spectroscopy and transmission electron microscopy. Spectroscopy results identified Orelated groups (i.e., $\mathrm{OH}, \mathrm{COOH}$, and $\mathrm{O}-\mathrm{CH}$ groups respectively) on the NDs surface (Figure 1a) and their manufacturing process as resulted from the oxidation of $\mathrm{sp} 2$ carbon during detonation soot purification[28]. Specifically, the peak at $3350 \mathrm{~cm}^{-1}$ was attributed to the $\mathrm{O}-\mathrm{H}$ stretching vibration, while the peak at around $1750 \mathrm{~cm}^{-1}$ was associated with the $\mathrm{C}=\mathrm{O}$ stretching vibration[18]. In addition, the small peak observed at around $1600 \mathrm{~cm}^{-1}$ was a result of the $\mathrm{O}-\mathrm{H}$ bending vibration, with the peaks between 1000 to $1250 \mathrm{~cm}^{-1}$ being associated with the $\mathrm{C}-\mathrm{O}$ stretching vibrations[29]. Electron microscopy investigations showed that NDs had planar structures that ranged from 3 to $7 \mathrm{~nm}$ in size, with an average of $5 \mathrm{~nm}$ (Figure 1b). Furthermore, the NDs formed primary agglomerates (conglomerates of individual NDs; Figure 1b insert) which ranged from 30 to $100 \mathrm{~nm}$ and were comparable to previous studies[30, 31].

Dispersity analyses performed using dynamic light scattering (DLS) (Figure 1c) showed that NDs dispersed in water had an average size of about $63.6 \pm 10.3 \mathrm{~nm}$ while the ones dispersed in cellular media reached $89.6 \pm 12.8 \mathrm{~nm}$. Their agglomeration is a result of the O-balance present at their synthesis as demonstrated by the negative zeta potential $(-28.2 \pm 2.4 \mathrm{mV})$. The larger size of these NDs in media was presumably due to additional serum proteins adsorbed onto their surfaces and the formation of protein corona that as demonstrated by the change in zeta potential $(-93.35 \pm 5.5 \mathrm{mV})$.

To explain the agglomeration and the changes in dispersity, we performed computational 
analyses (Figure 1d). We considered neutral NDs for simulations and subsequently evaluated their agglomeration in the presence of serum proteins, with bovine serum albumin (BSA) being the dominant one. The consideration for neutral NDs as compared to anionic ones come from previous studies which showed that larger protein concentrations in the media would lead to overall minor variations in zeta potentials to influence their cellular uptake[32, 33]. Complementarily, the consideration for BSA was based on previous studies showing that concentrations of up to $27 \mathrm{mg} / \mathrm{ml} \mathrm{BSA}$ are present in certified fetal bovine serum (FBS), resulting in an approximate $1.35 \mathrm{mg} / \mathrm{ml}$ in DMEM media containing 5\% FBS (v/v)[34]. Our approach also took into account that most cellular membranes are negatively charged thus further influencing the local environment of the ND and their uptake.

The BSA protein being aspherical and with cross-sectional lengths of 9.1, 6.4 and $5.1 \mathrm{~nm}$ respectively, is comparable with or larger than the NDs used in this work. Analysis showed that when the BSA was interacted with the neutral NDs, an individual BSA-ND complex was formed, all within $200 \mathrm{~ns}$ of the simulation, with the complex formation being mainly driven by van der Waals and hydrophobic interactions between the neutral ND and negatively charged BSA molecules respectively. Results also showed that only a fraction of the BSA surface could contribute to the formation of the complex. As such, a stable non-uniform monolayer of BSA molecules would be observed for all ND sizes being tested with the monolayer leading to an increase in the hydrodynamic radius of the NDs or resulting complexes (Figure 1d). For instance, the snapshot of $3 \mathrm{~nm}$ ND showed a non-uniform protein corona being formed onto its surface, with about 3 BSA molecules being adsorbed as blobs with pockets to lead to a complex of a $8.6 \pm$ $2.1 \mathrm{~nm}$ diameter. Non-uniform protein coronas were also observed for both 5 and $7 \mathrm{~nm}$ ND sizes resulting in $13.1 \pm 3.2$ and $15.7 \pm 4.9 \mathrm{~nm}$ diameters, respectively. In control, analyses with 
negatively charged particles (Supporting information; S1) also led to a non-uniform protein corona formation as resulted from a maximum adsorption of about 4 BSA molecules onto larger particles. Taking into account that the stoichiometry of the BSA-ND complex is sensitive to several physicochemical properties that include NDs size, ND surface charge, protein-protein interactions, charge on the protein, as well as the $\mathrm{pH}$ of the medium, the variation in protein corona stoichiometry is minimal relative to the protein corona formed for the neutral particle and represents the result of the continuous physical exchange at ND interfaces[35].

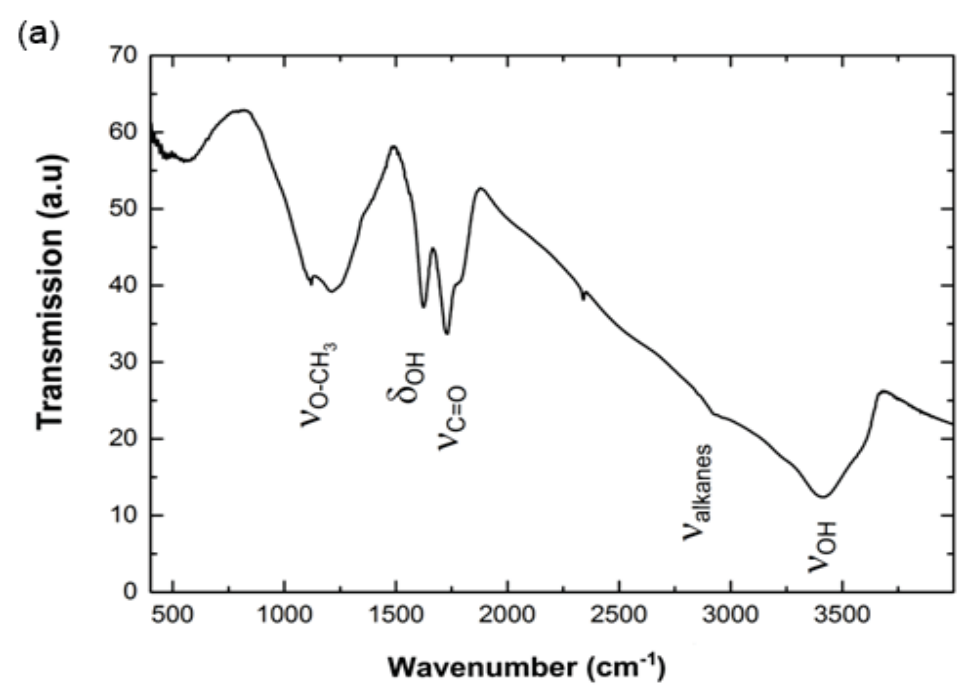

(b)

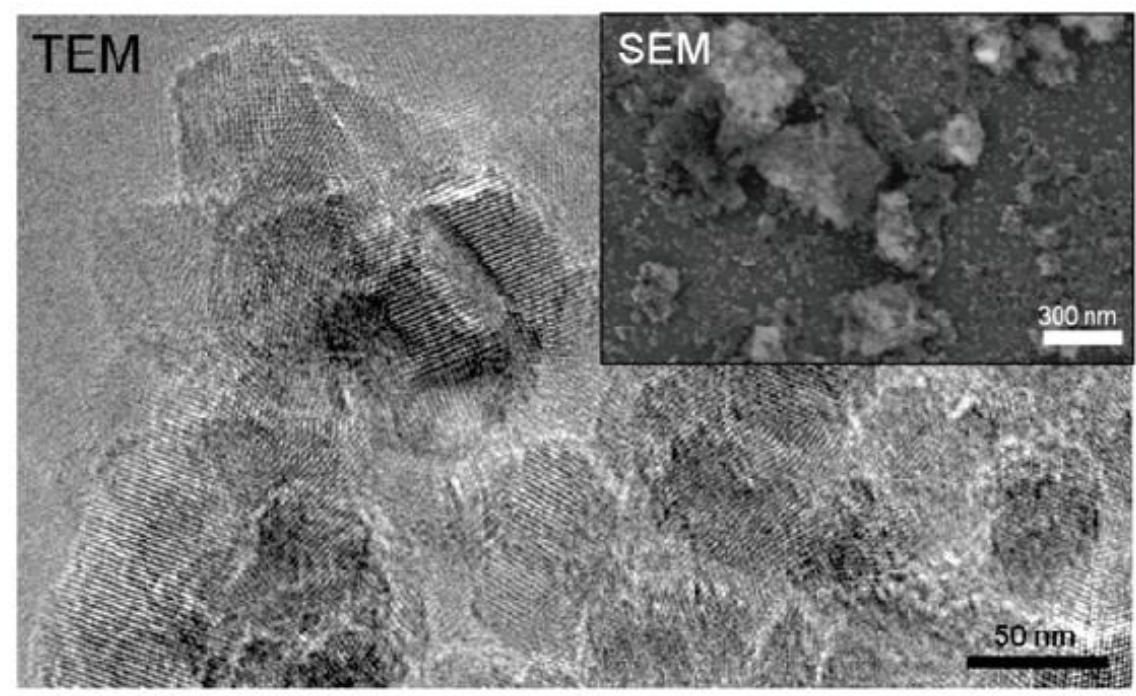


(c)

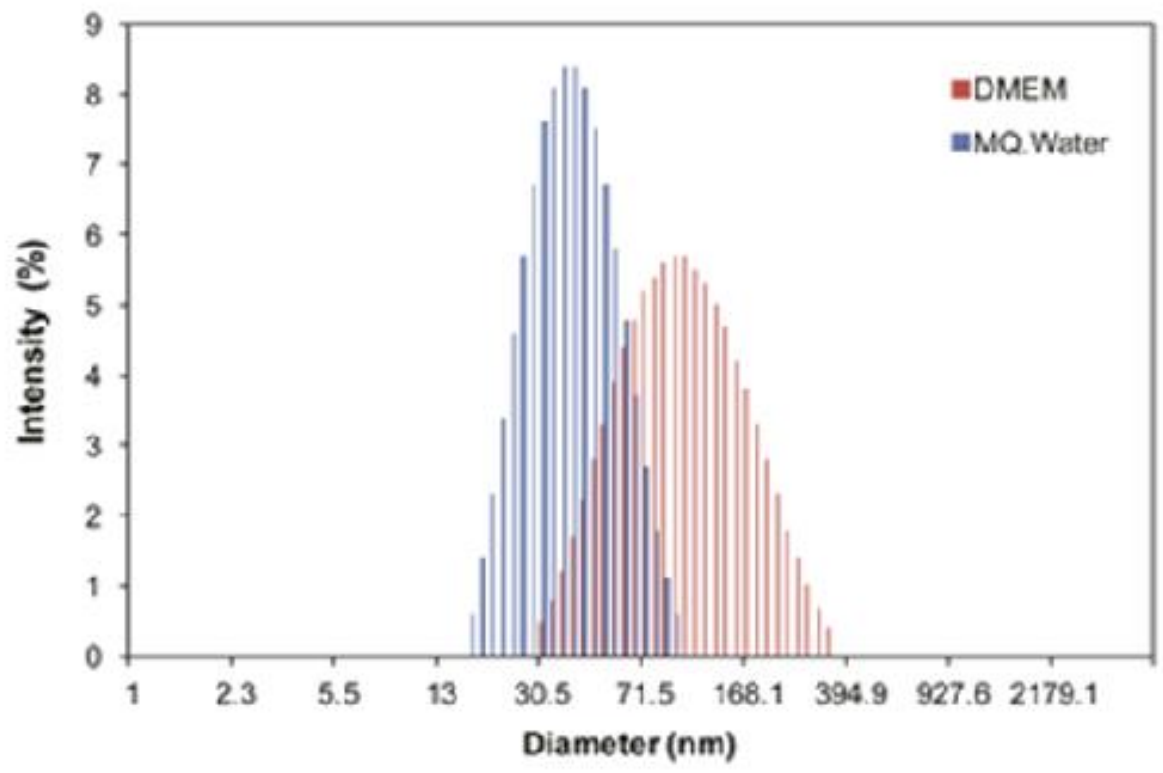

(d)

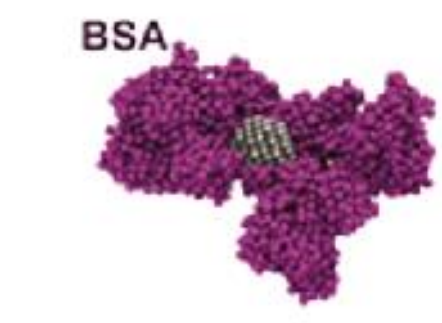

ND $\quad 3 \mathrm{~nm}$

ND-BSA $\quad 8.6+-2.1 \mathrm{~nm}$

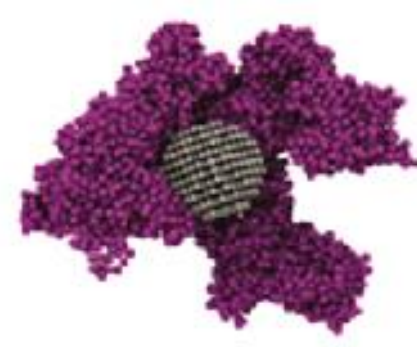

$5 \mathrm{~nm}$

$13.1+-3.2 \mathrm{~nm}$

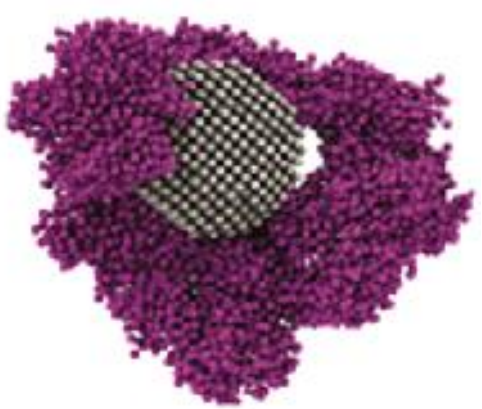

$7 \mathrm{~nm}$

$15.7+-4.9 \mathrm{~nm}$

Figure 1: Characterization of detonation nanodiamonds (NDs), (a) Fourier Transform Infrared spectra indicating the presence of O-rich groups. (b) Transmission and Scanning Electron microscope images (TEM and SEM in insert respectively). (c) Dynamic light scattering (DLS) intensity distribution showing the average agglomerate diameter of NDs. 


\section{ND uptake and induced cellular biocompatibility}

We confirmed the uptake of NDs using their intrinsic photoluminescence and standard in vitro fluorescence activated cell sorting (FACS) analysis. Briefly, human lung epithelial cells (BEAS-2B) were exposed to NDs dispersed in cellular media containing BSA for 1, 2, 3 and $24 \mathrm{~h}$ respectively; BEAS-2B previously served as a suitable model system for in vitro testing of uptake of carbon nanotubes[18], titanium dioxide[36], and silver nanoparticles[37] just to name a few. Figure 2a shows that NDs cellular uptake was function of both the concentration and the exposure time, with analyses revealing that cells exposed to concentrations of 400, 200 and $100 \mu \mathrm{g} / \mathrm{ml} \mathrm{NDs}$ had significantly higher fluorescence intensity relative to their control counterparts (i.e., unexposed cells). Fluorescent signals of cells exposed to 50 and $25 \mu \mathrm{g} / \mathrm{ml}$ NDs were however not significantly different from their control counterparts. Control experiments did not identify large conglomerates or particles thus confirming that the NDs uptake was in its majority.

The uptake did not influence the cell structure or morphology; this was confirmed experimentatlly using real-time non-invasive electric cell impedance-sensing (ECIS) experiments. Specifically, we used the insulating nature of the plasma membrane and ECIS to provide sensitive analysis of membrane fluctuations or cytoskeleton reorganization[38-41]. The ECIS was previously employed to study cellular viability, proliferation[42], motion[40], morphology[39] and attachment[20] post exposure to different toxins[42], therapeutic agents or viral vectors[42], and nanomaterials of carbon[43]. Figure $2 \mathrm{~b}$ shows representative ECIS measurements of the normalized resistance values for control and NDs-exposed cells performed for $48 \mathrm{~h}$ at $4 \mathrm{kHz}$ frequency; the selected operational frequency was previously shown to allow for optimal measurement of the cellular membrane integrity, cellular adhesion and cell-cell interactions over the gold electrodes[38, 41]. 
Our results revealed that the resistance curves of the NDs-exposed cells were similar to those of the control cells and showed no changes in cellular morphology or integrity of the cellular monolayer. Furthermore, analyses showed that there were no quantifiable differences in the fluctuations of the resistance curves, which confirmed that the uptake of NDs did not lead with observable membrane changes or cellular-induced micromotion onto electrodes[40, 44] (Figure 2c).

(a)

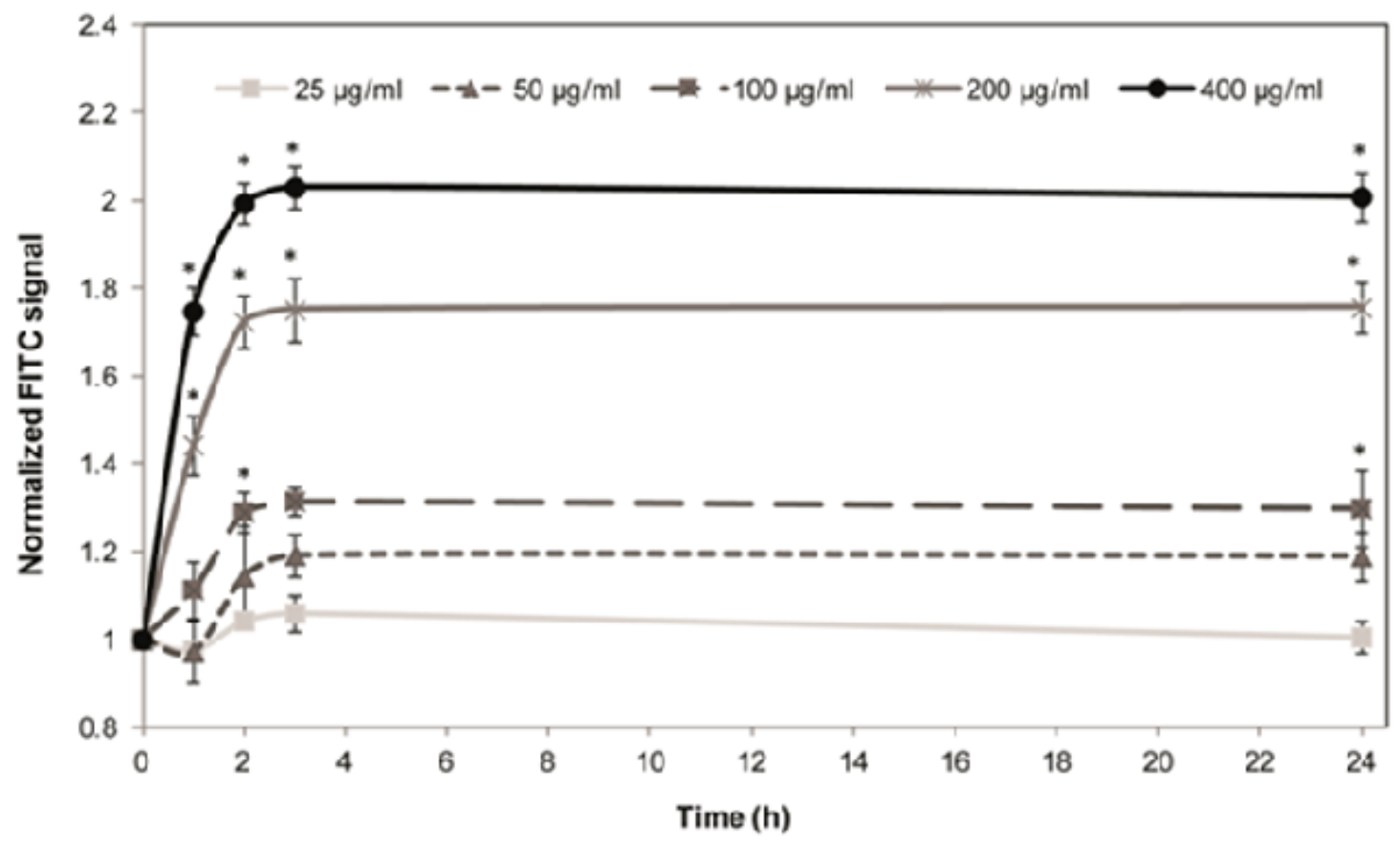


(b)

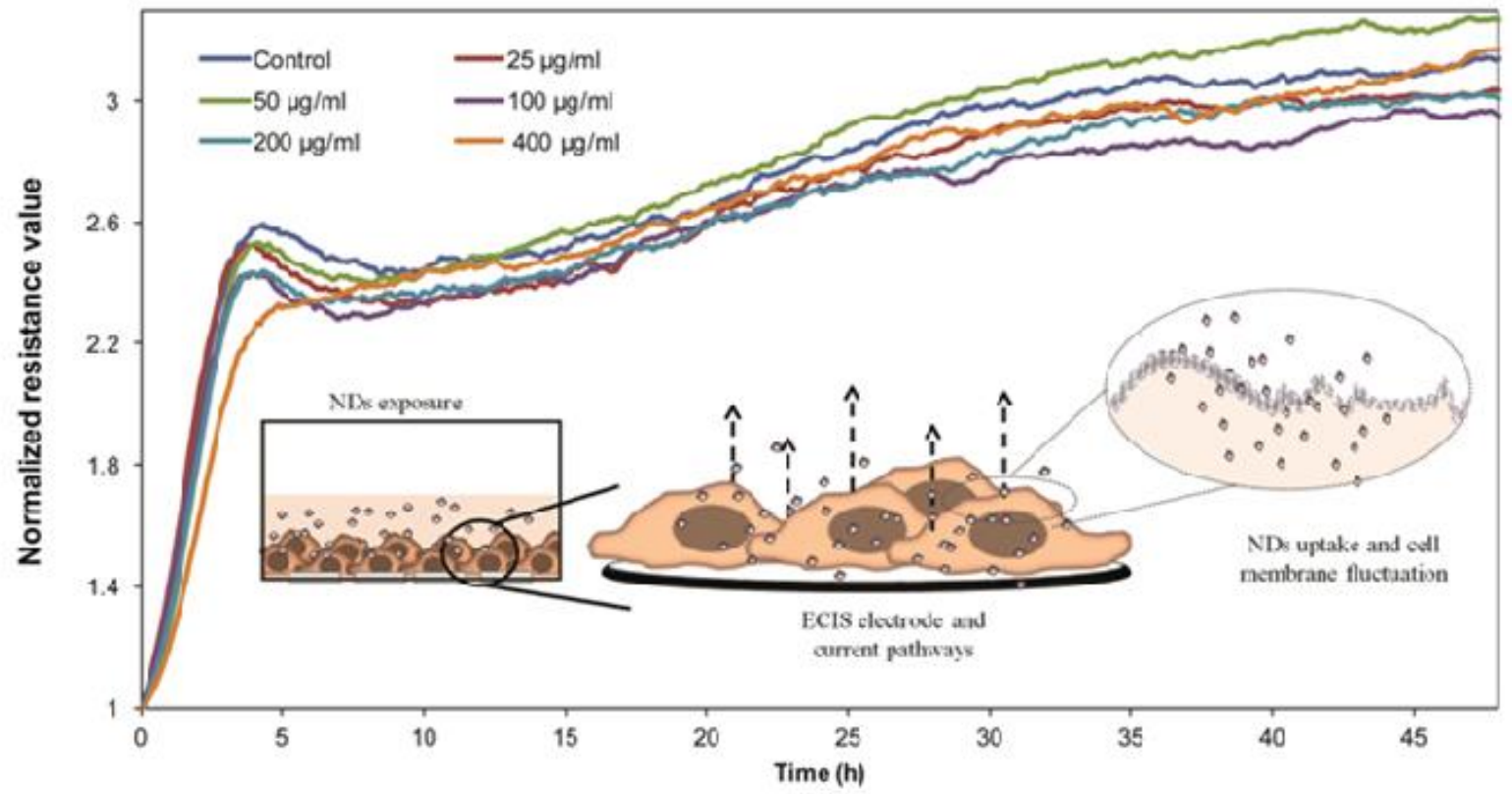

(c)

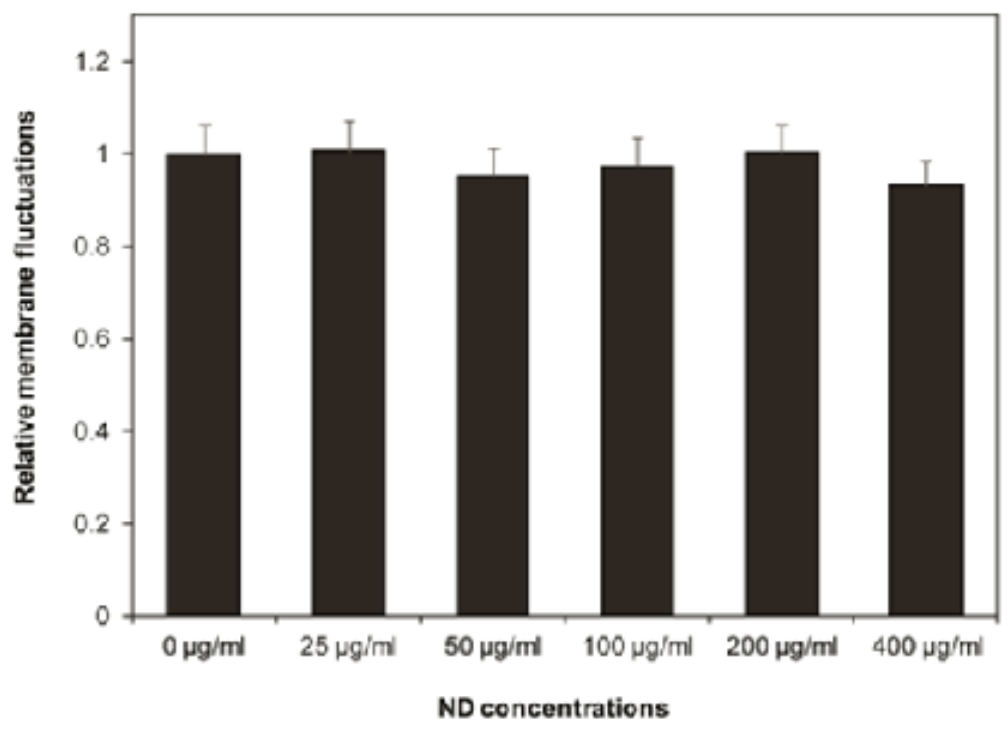

Figure 2: ND uptake and induced cellular biocompatibility following NDs exposure. (a) Normalized FITC signal of cells exposed to $(25,50,100,200,400 \mu \mathrm{g} / \mathrm{ml})$ NDs for 1, 2, 3 and $24 \mathrm{~h}$ respectively. (b) Representative normalized resistance measurements of BEAS- 
2B cells upon their exposure to different concentrations of NDs, (c) Quantifications of membrane fluctuations of cells exposed to NDs relative to unexposed cells. Differences were considered statistically significant for $\mathrm{p}^{*}<0.05$.

Upon confirming NDs uptake, we validated their induced biocompatibility through analysis of cellular activity and cell cycle progression respectively. Cellular activity is regulated by different organelles such as the mitochondria, plasma membrane, as well as by cell-cell interactions and is a reflection of the overall cell fate[45]. Cell cycle controls proliferation, differentiation, and apoptosis processes[46], with changes in the cell cycle phases being associated with cyto- and/or genotoxic effects observed upon exposure to different analytes as well as cellular transformation to a malignant phenotype[47].

Cellular activity analyses at discrete time points using colorimetric detection based on WST-1 assay[21] showed that $24 \mathrm{~h}$ exposure to different concentrations of NDs did not induce significant effects on cell viability (Figure 3a), thus confirming previous studies[13, 48]. Complementary, cell cycle analyses performed by measuring the percentage of the DNA content showed that NDs exposure did not alter cell cycle progression nor interfered with cellular proliferation (Figure 3b). 

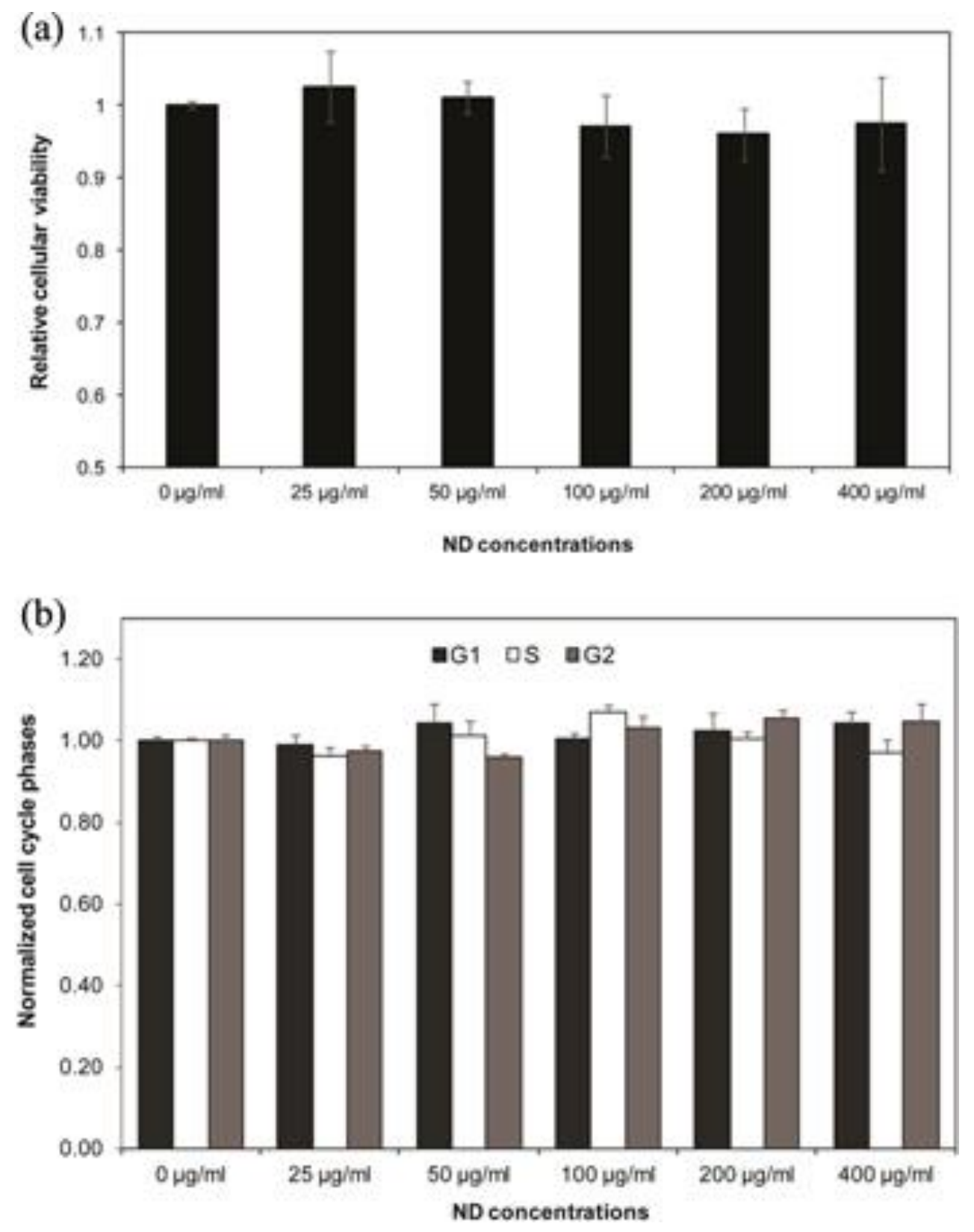

Figure 3: (a) Viability analysis following exposure to NDs for $24 \mathrm{~h}$ relative to control (unexposed) cells. (b) Cell cycle analysis following $24 \mathrm{~h}$ exposure to NDs. All differences were considered statistically significant for $\mathrm{p}^{*}<0.05$. 


\section{Discussion}

We used experimentally-validated multiscale modeling and a cell-based sensing platform to evaluate real-time uptake of detonation nanodiamonds (NDs). The consideration for NDs came from their potential applicability for the delivery of genes and drugs as resulted from their ease of biomolecular functionalization and slow release capabilities[5, 30]. Our multi-scale strategy involved first characterizing the physico-chemical properties of user-produced NDs, secondly validating their uptake in model cellular systems, and thirdly providing our hypothesis to explain their increased biocompatibility in such systems.

Our analyses showed that even though a narrow size distribution for the synthesized NDs was recorded, upon their dispersion in cellular media their agglomeration state has changed. The change in the agglomeration state was presumably due to the attachment of the proteins present in the media onto their interfaces. Specifically, previous studies showed that the composition and size of such proteins could lead to protein corona formation, with the size of the corona being dependent on the concentration, the affinity, as well as the interaction of these proteins with the interfaces they are exposed to[49] [50].

The detailed analysis of the interactions between the ND and a representative protein present in the media, namely BSA, showed non-specific binding of its amino acid residues onto the ND surface. Furthermore, the asymmetry of the protein sterically seemed to hinder the addition of other protein molecules in any of the exposed NDs pockets. As such, the remaining BSA surface area would presumably be available to facilitate the agglomeration of the NDs as evident from the experimental DLS data. In particular, using the value ratios of individual NDs and their average size with and without the protein corona, the packing density would be function of the dispersion 
media, i.e. water or DMEM media respectively, with a smaller number of NDs being packed in the media presumably due to the formation of thin electric dipole layers of the protein corona that will affect the hydrodynamic diameter of the NDs[51].

Our analysis showed that the majority of the NDs uptake occurred within the first $2 \mathrm{~h}$ of exposure, with cells subsequently reaching a "saturation" plateau. In particular, mathematical modeling of the fluorescent signal of cells exposed to NDs at concentrations above $200 \mu \mathrm{g} / \mathrm{ml}$ showed that uptake followed a 3-parameters exponential curve according to the following equation:

$$
I(t)=A\left[1-e^{k\left(t_{c}-t\right)}\right],
$$

Where $I(t)$ represents the mean fluorescence intensity at the particular time, $A$ is the saturation value of mean fluorescence intensity, $k$ is the rate constant, and $t_{c}$ is the lag in particle diffusion and targeting. The model also showed that about $95 \%$ of the NDs where internalized within the $1.71 \mathrm{~h}$ of incubation $\left(\mathrm{R}^{2}=0.95\right)$ with such analysis being consistent with previously published results investigating NDs uptake in a serum-rich medium[52].

While a clear discrepancy was recorded in the literature on the uptake and induced cellular fate upon other carbon-based nanomaterials such as single walled (SW-), and multi-walled carbon nanotubes (MWCNTs), with the conflicting data being attributed to the differences in the preparation procedures, intrinsic purities and activities of such nanomaterials, our study confirms the high cellular biocompatibility observed upon NDs uptake[53]. Specifically, previous studies showed that NDs functionalized with O-containing groups (either $-\mathrm{OH}$ or $-\mathrm{CO}_{2} \mathrm{H}$ groups) might lead to complex membrane-particle interactions[54] and their nonspecific binding or clustering on the cationic sites of the plasma membrane, with subsequent endocytosis through a temperature-, energy-, and clathrin-dependent pathway[54-57]. The uptake of NDs is in contrast with both the 
uptake of SW- and MWCNTs respectively shown to lead to membrane damage, association with cytoskeleton structures and increased disruption of cellular integrity and organelles upon their translocation $[18,58]$.

Since our results showed that the NDs uptake did not change cell activity nor affect its cycle, we hypothesized that the observed biocompatibility is a result of minimal membrane damage induced upon their uptake. Our hypothesis takes into account the role of the cellular membrane to serve as a gate that regulates cell activity and structure through direct association with cellular organelles and cytoskeletal components. In particular, if the NDs would have had disrupted the membrane, such a disruption would have had resulted in "membrane pinches" possibly accompanied by cytoskeletal reorganization to affect the overall integrity of the cell and lead to apoptosis or necrosis $[24,34,45]$. Indeed, previous membrane passive penetration mechanisms were shown to affect cell fate leading to the formation of holes and a significant membrane disruption[59, 60]. In contrast, energy-dependent cellular uptake was not shown lead to such effects.

To validate our hypothesis we performed additional coarse-grained (CG) computational analyses. Our results showed that the size of the NDs or induced conglomerates played a critical role in the internalization process, with the maxima and minima in their energy profiles providing direct evidence of the lack of membrane disruption. Specifically, the potential of mean force (PMF) profiles for the three representative sizes of NDs as demonstrated by the TEM analysis, showed that key features such as the distance between the center-of-mass (COM) of ND and membrane mid-plane was reduced. Further, we found that as the $3 \mathrm{~nm}$ NDs get closer to the cellular membrane, the uptake energy increases as less favorable interactions between the uncharged NDs and the negatively charged polar head groups of the lipid membrane would take place (Figure 4a). 
However, past the lipid heads and as the NDs get embedded in the membrane, the hydrophobic lipid tails interact favorably to stabilize the individual particles leading to energies as high as 60 $\mathrm{kJ} / \mathrm{mol}$. In contrast, the simulations showed that the $5 \mathrm{~nm}$ NDs had no minima when embedded in the lipid chains since the hydrophobic thickness of the membrane was smaller than the diameter of the ND (Figure 4b). A further increase in diameter to $7 \mathrm{~nm}$ respectively would have led to pronounced deformations of the membrane structure causing an increase of about 14-fold in the energy barrier relative to the $5 \mathrm{~nm}$ ND (Figure 4c). However, the lack of membrane fluctuations recorded by ECIS confirmed that there were no changes on the membrane integrity thus validating our above proposed hypothesis.

(a)

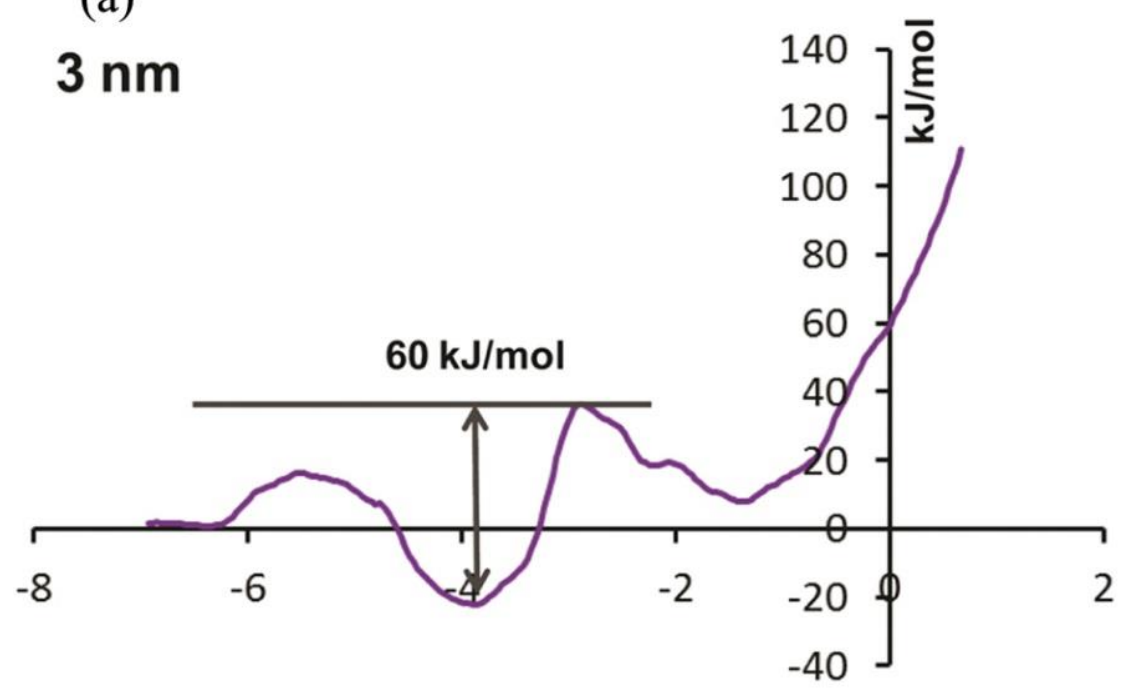

Distance between ND COM to membrane mid-plane 


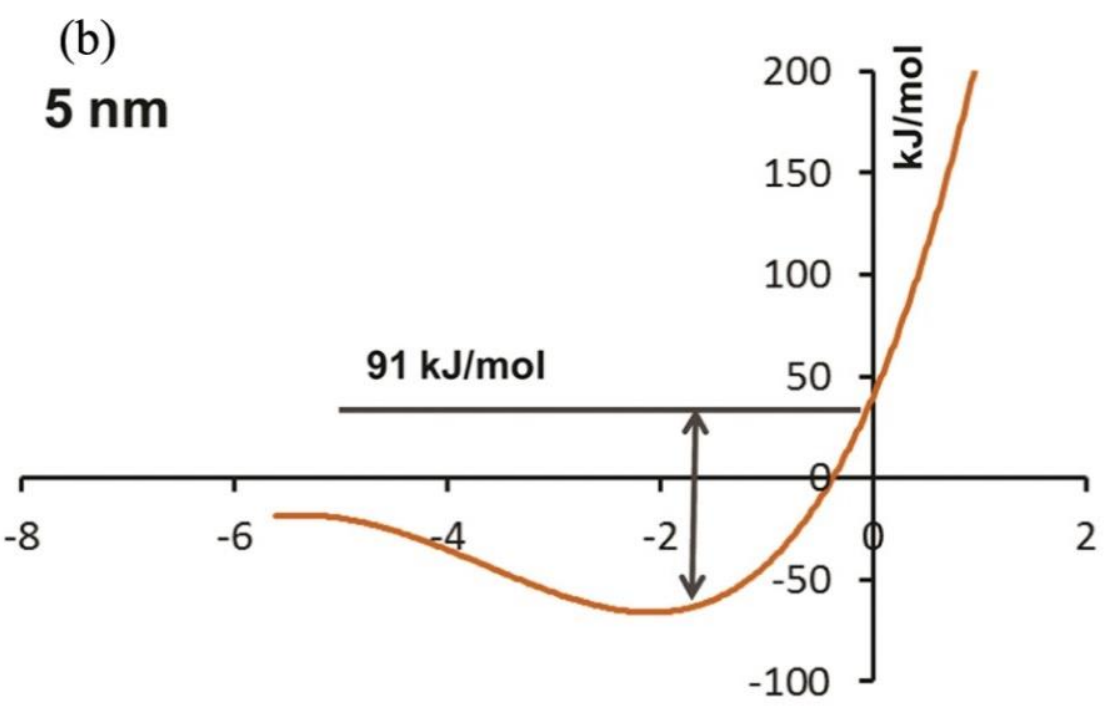

Distance between ND COM to membrane mid-plane

(c)

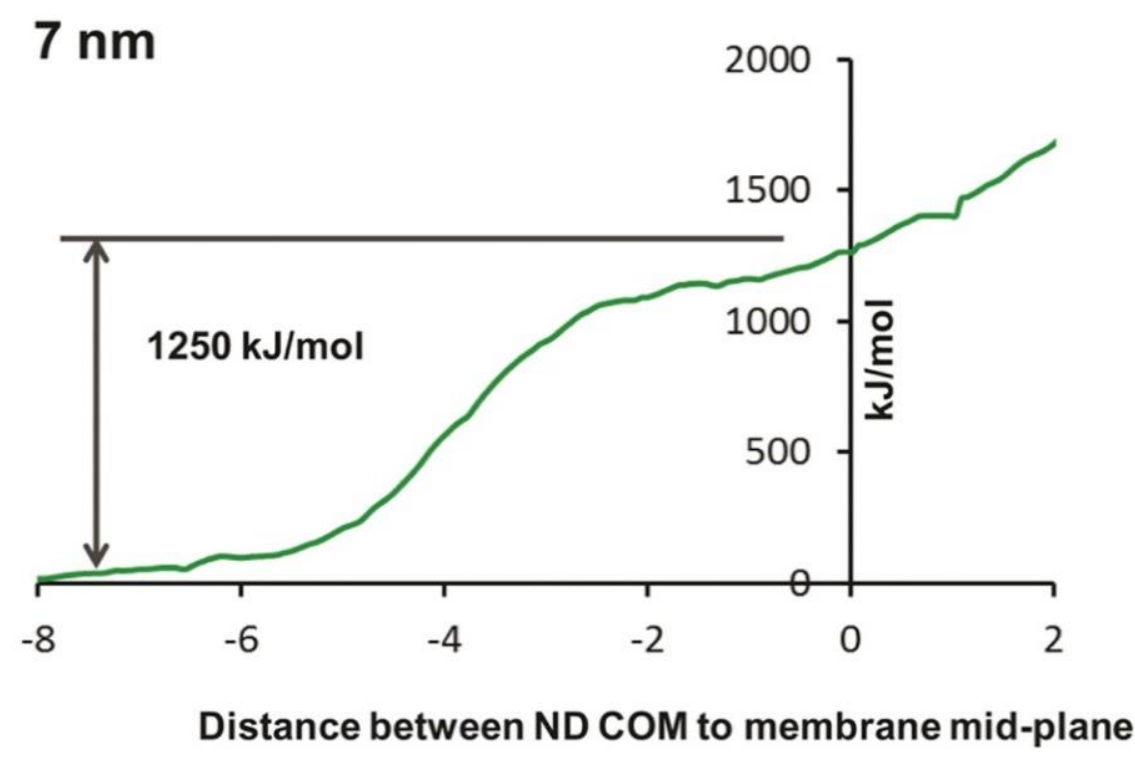

Figure 4: Coarse-grained (CG) computational analysis of the potential of mean force (PMF) profiles for the $3 \mathrm{~nm}(\mathrm{a}), 5 \mathrm{~nm}$ (b) and $7 \mathrm{~nm}$ (c) NDs as function of the distance between the center-of-mass (COM) of NDs and membrane mid-plane.

Snapshots further showed that if the uptake process would have occurred via only translocation a clear deformation in the cell membrane for the particles being investigated would 
have been observed (Figure 5). The membrane recovery time would have correlated directly with the damage caused by the ND translocation, with the membrane lipid self-assembling into a stable bilayer within $50 \mathrm{~ns}$ of the simulations for both the 3 and $5 \mathrm{~nm}$ NDs while the rate constant of the translocation was appreciable resulting in a half-life of $1.4 \mathrm{~h}$ for the $5 \mathrm{~nm}$ diameter particle (Table 1).

$3 \mathrm{~nm}$
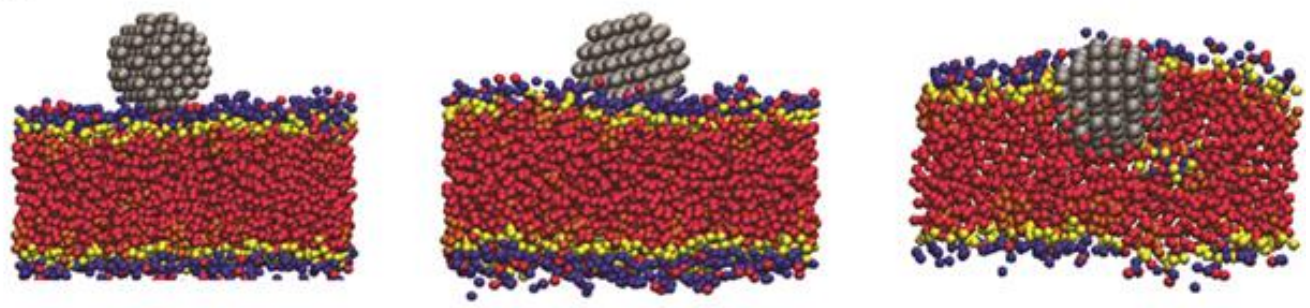

$5 \mathrm{~nm}$
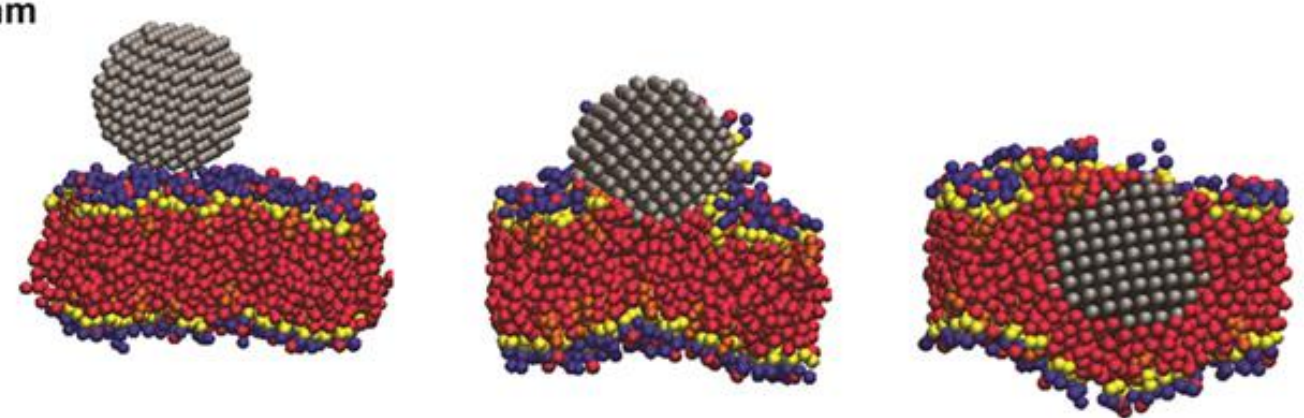

$7 \mathrm{~nm}$
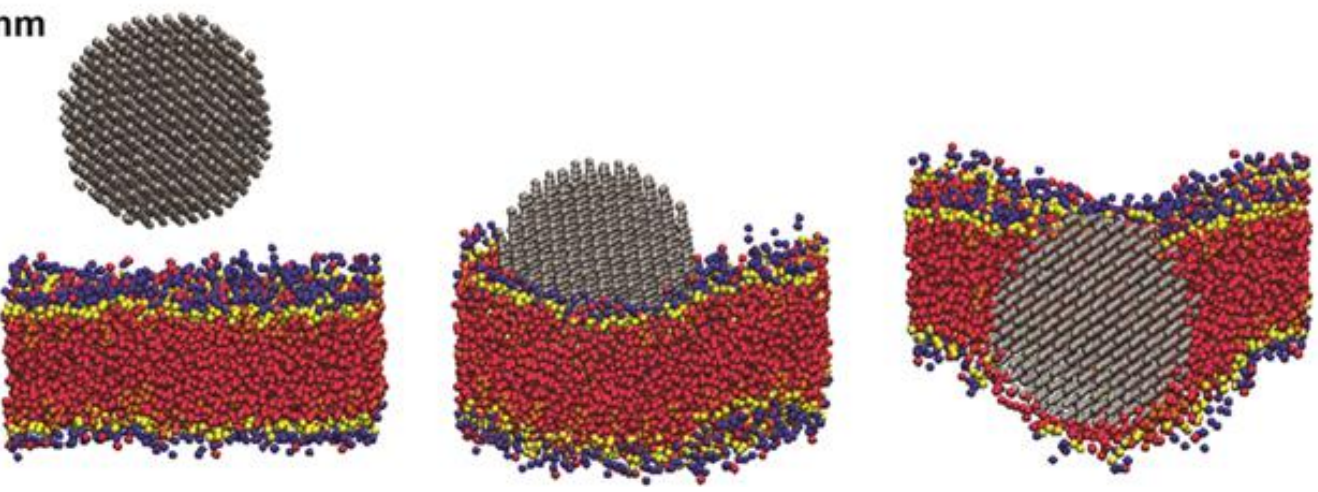

Figure 5: Snapshots revealing the translocation of NDs with different diameter (i.e., 3, 5 and 7 $\mathrm{nm}$ respectively) through the membrane lipid bilayer. The images showed the membrane disruption in response to the NDs. 
Table 1: Calculated barrier heights, rate constants and half-lives for cellular uptake of NDs without BSA protein corona

\begin{tabular}{cccc}
\hline $\begin{array}{c}\text { ND diameter } \\
(\mathrm{nm})\end{array}$ & Barrier height & $k$ & $\mathrm{t}_{1 / 2}$ \\
& $(\mathrm{~kJ} / \mathrm{mol})$ & $\left(\mathrm{h}^{-1}\right)$, & $(\mathrm{h})$ \\
\hline 3 & $60 \pm 1$ & $6.5 \times 10^{5}$ & $1.5 \times 10^{-6}$ \\
5 & $91 \pm 3$ & $4.9 \times 10^{-1}$ & 1.4 \\
7 & $1250 \pm 12$ & & \\
& & $\mathrm{NA} *$ & $\mathrm{NA}^{*}$ \\
\hline
\end{tabular}

*NA-Not applicable. There appears to be a change in the cellular uptake mechanism for NDs with size greater than $5 \mathrm{~nm}$.

Our study demonstrates that the lack of membrane disruption can be recorded through multi-scale combinatorial, non-destructive and high throughput velocity analyses, all performed in real-time and in contrast with previously destructive cellular endpoint assays. By unraveling the cellular biocompatibility on the basis of limited membrane and cellular disruption the current study has the potential to advance the implementation of NDs for the next generation of nanovehicles used for drug or gene delivery. 


\section{Conclusions}

Our results correlate the free-energy barrier, half time and rate constant of NDs uptake with the average size of the NDs in order to explain their increased cellular biocompatibility. Furthermore, our discrete and real-time analyses of the cellular viability, proliferation and overall cellular behavior upon NDs uptake help explain the lack of membrane disruption and allow for high-throughput evaluation of the cell fate through meaningful combinatorial approach with potential to expand testing of other nanomaterial's biocompatibility.

\section{Acknowledgements}

Support for this research was provided through the National Science Foundation (NSF 1434503, 1300757 and EPS-1003907). Flow cytometry experiments were performed in the Flow Cytometry Core Facility at WVU; the Core Facility is in part supported by the National Institute of Health equipment grant number S10OD016165 and the Institutional Development Award (IDeA) from the National Institute of General Medical Sciences of the National Institutes of Health under grant numbers P30GM103488 (CoBRE) and P20GM103434 (INBRE). SEM measurements were performed by Yvonne Standke at IKTS; DLS measurements were performed at the Physical Chemistry Department of the TU Dresden. The computational work used the Extreme Science and

Engineering Discovery Environment (XSEDE), which is supported by National Science Foundation grant number ACI-1053575. The financial support for the computational studies was provided by Syracuse University. 


\section{Supporting Information}

\section{Chapter 2: Combinatorial Approaches to Evaluate Nanodiamonds Uptake and Induced Cellular Fate}

\section{Materials and Methods}

\section{Simulation setup of negatively charged nanodiamonds (NDs)}

To evaluate the uptake of NDs we used coarse-grained representation and the GROMACS software package (v4.5.5). Atomistic models of $\mathrm{NDs}(3,5$, and $7 \mathrm{~nm}$ in diameter) were generated using the diamond lattice (with Fd3m space group and face-centered cubic lattice) in CrystalMaker software. The coarse-grained representation was developed using the MARTINI four-to-one mapping scheme. Coarse grained ND beads were given standard C1 bead type and a zero net surface charge. Subsequently, the ND was energy minimized and equilibrated with explicit MARTINI water (isobaric-isothermal ensemble at $300 \mathrm{~K}$ and 1 atm pressure).

The coarse-grained lipid bilayer used for the analysis was made up of neutral distearoylphosphatidyl choline (DSPC) molecules and negatively charged distearoylphosphatidyl glycerol (DSPG) lipid molecules. The DSPC:DSPG ratio was maintained at a 3:1 ratio to resemble a net negative charge of mammalian cell membranes. The electroneutrality of the total system was achieved by adding the required number of sodium counterions as determined by the number of DSPG lipid molecules being available. The bilayer was allowed to self-assemble without any constraints in a user-designed simulation box with a typical size of $16.0 \times 16.0 \times 30 \mathrm{~nm}^{3}$. In all simulations the center-of-mass $(\mathrm{COM})$ of the $\mathrm{ND}$ was placed $7.0 \mathrm{~nm}$ along the $+\mathrm{z}$-axis above the lipid bilayer as the starting structure for the simulation; the entire simulation box was solvated with coarse-grained water beads of which $10 \%$ were anti-freeze type. 
To mimic the experimental serum protein in medium, bovine serum albumin (PDB: 4F5S) protein was used in the simulations. Bovine serum albumin (BSA) is one of the most extensively utilized proteins in laboratory experiments. High resolution $(2.47 \mathrm{~nm})$ BSA crystal structure was obtained from the protein data bank and coarse-grained using the available MARTINI scripts. The secondary structure of the protein and the bead assignment were based on standard MARTINI coarse grained methods. The interactions between coarse grained BSA and NDs of sizes 3,5 , and $7 \mathrm{~nm}$ were investigated in three separate simulations, where ND surface beads were assigned $\mathrm{Q}_{0}$ bead type with a fractional charge of -0.432 to mimic the experimental conditions and $\mathrm{pH}$, whereas core beads were assigned a $\mathrm{C} 1$ bead type and a zero charge. In each case, the NDs were introduced in a well-equilibrated system of BSA molecules with explicit water and positively charged counter ions $\left(\mathrm{Na}^{+}\right)$at $300 \mathrm{~K}$. The counter ions were added to maintain the electroneutrality of the simulation system because BSA is a negatively charged protein under the physiological conditions.

\section{Results and discussion}

Analyses of the interactions of negatively charged NDs with serum proteins were perfomed using grain-coarse model. For this, we used bovine serum albumin (BSA) as model protein, based on its high presence in the media supplemented with FBS. Analysis showed that when BSA interacts with negatively charged NDs, protein dense coronas are being formed instantaneously leading to the formation of a stable non-uniform monolayer of BSA molecules and an increase in the hydrodynamic radius of the NDs (Supporting Figure1). For instance, the snapshot of $3 \mathrm{~nm}$ ND showed a non-uniform protein corona being formed onto its surface, with 3 BSA molecules being adsorbed as blobs with pockets to lead to a complex with $11.2 \pm 1.9 \mathrm{~nm}$ diameter. Similarly, NDs with 5 and $7 \mathrm{~nm}$ particle sizes lead to the formation of corona complexes that were around $14.1 \pm$ 1.5 and $17.1 \pm 4.8 \mathrm{~nm}$ diameters, respectively. The recorded sizes are supporting the hypothesis 
that the stoichiometry of the BSA-ND complex is sensitive to several physico-chemical properties that include NDs size, ND surface charge, protein-protein interactions, charge on the protein, as well as the $\mathrm{pH}$ of the medium. However, this variation in protein corona stoichiometry is minimal relative to the protein corona formed for the neutral particle and represents the result of the continuous physical exchange at ND interfaces to influence the overall charge density.

\section{Supporting Figure 1}

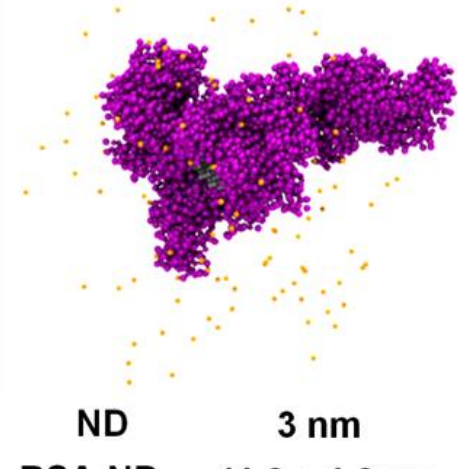

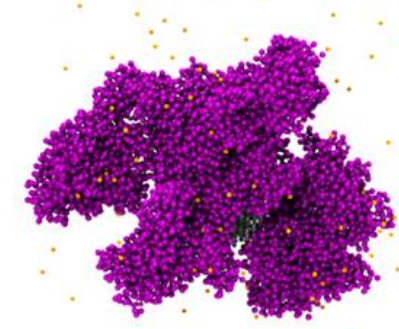

$5 \mathrm{~nm}$

$14.1 \pm 1.5 \mathrm{~nm}$

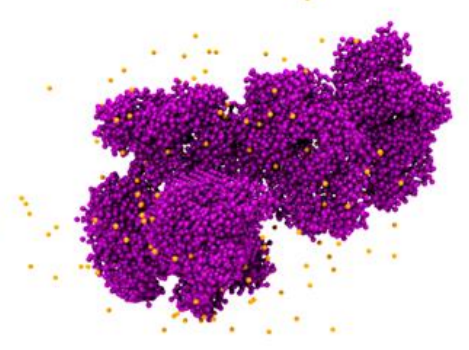

$7 \mathrm{~nm}$

$17.1 \pm 4.8 \mathrm{~nm}$

Snapshots of ND-BSA composite particles showing size measurements of individual NDs as well as ND-protein complexes. Colors: ND (gray); BSA (purple); positively charged counterions (orange). 


\section{References}

1. Shenderova, Olga A., 3.07 - Production of Nanodiamond Particles, in Comprehensive Hard Materials, V.K. Sarin, Editor 2014, Elsevier: Oxford. p. 143-171.

2. Schrand, Amanda M., Suzanne A. Ciftan Hens, and Olga A. Shenderova, Nanodiamond Particles: Properties and Perspectives for Bioapplications. Critical Reviews in Solid State and Materials Sciences, 2009. 34(1-2): p. 18-74.

3. Mansoorianfar, Mojtaba, Mohammad Ali Shokrgozar, Morteza Mehrjoo, Elnaz Tamjid, and Arash Simchi, Nanodiamonds for surface engineering of orthopedic implants: Enhanced biocompatibility in human osteosarcoma cell culture. Diamond and Related Materials, 2013. 40(0): p. 107-114.

4. Perevedentseva, E., P. J. Cai, Y. C. Chiu, and C. L. Cheng, Characterizing Protein Activities on the Lysozyme and Nanodiamond Complex Prepared for Bio Applications. Langmuir, 2010. 27(3): p. 1085-1091.

5. Grichko, Varvara P. and Olga A. Shenderova, 15 - Nanodiamond: Designing the BioPlatform, in Ultrananocrystalline Diamond, O.A.S.M. Gruen, Editor 2006, William Andrew Publishing: Norwich, NY. p. 529-557.

6. Perevedentseva, E., Y. C. Lin, M. Jani, and C. L. Cheng, Biomedical applications of nanodiamonds in imaging and therapy. Nanomedicine (Lond), 2013. 8(12): p. 2041-60. 
7. Kuang-Kai, Liu, Cheng Chia-Liang, Chang Chia-Ching, and I. Chao Jui, Biocompatible and detectable carboxylated nanodiamond on human cell. Nanotechnology, 2007. 18(32): p. 325102 .

8. Zhu, Ying, Jing Li, Wenxin Li, Yu Zhang, Xiafeng Yang, Nan Chen, Yanhong Sun, Yun Zhao, Chunhai Fan, and Qing Huang, The Biocompatibility of Nanodiamonds and Their Application in Drug Delivery Systems. Theranostics, 2012. 2(3): p. 302-312.

9. Schrand, Amanda M., Liming Dai, John J. Schlager, Saber M. Hussain, and Eiji Osawa, Differential biocompatibility of carbon nanotubes and nanodiamonds. Diamond and Related Materials, 2007. 16(12): p. 2118-2123.

10. Pulmonary toxicity and translocation of nanodiamonds in mice. Diamond and Related Materials, 2010. 19(4): p. 291.

11. Puzyr, A. P., A. V. Baron, K. V. Purtov, E. V. Bortnikov, N. N. Skobelev, O. A. Mogilnaya, and V. S. Bondar, Nanodiamonds with novel properties: A biological study. Diamond and Related Materials, 2007. 16(12): p. 2124-2128.

12. Kumari, S., M. K. Singh, S. K. Singh, J. J. Gracio, and D. Dash, Nanodiamonds activate blood platelets and induce thromboembolism. Nanomedicine (Lond), 2014. 9(3): p. 42740.

13. Vairakkannu, Vaijayanthimala, Tzeng Yan-Kai, Chang Huan-Cheng, and Li ChungLeung, The biocompatibility of fluorescent nanodiamonds and their mechanism of cellular uptake. Nanotechnology, 2009. 20(42): p. 425103. 
14. Perevedentseva, E., S. F. Hong, K. J. Huang, I. T. Chiang, C. Y. Lee, Y. T. Tseng, and C. L. Cheng, Nanodiamond internalization in cells and the cell uptake mechanism. Journal of Nanoparticle Research, 2013. 15(8): p. 1-12.

15. Zhang, Xiaoyong, Wenbing Hu, Jing Li, Lei Tao, and Yen Wei, A comparative study of cellular uptake and cytotoxicity of multi-walled carbon nanotubes, graphene oxide, and nanodiamond. Toxicology Research, 2012. 1(1): p. 62-68.

16. Vasir, Jaspreet K. and Vinod Labhasetwar, Quantification of the Force of NanoparticleCell Membrane Interactions and Its Influence on Intracellular Trafficking of Nanoparticles. Biomaterials, 2008. 29(31): p. 4244-4252.

17. Nangia, Shikha and Radhakrishna Sureshkumar, Effects of nanoparticle charge and shape anisotropy on translocation through cell membranes. Langmuir, 2012. 28(51): p. 1766617671.

18. Dong, Chenbo, Reem Eldawud, Linda M. Sargent, Michael L. Kashon, David Lowry, Yon Rojanasakul, and Cerasela Zoica Dinu, Towards elucidating the effects of purified MWCNTs on human lung epithelial cells. Environmental Science: Nano, 2014.

19. Dieguez, Lorena, Nasser Darwish, M Mir, Elena Martinez, M Moreno, and Josep Samitier, Effect of the refractive index of buffer solutions in evanescent optical biosensors. Sensor letters vol. 7, no. 5, 2009: p. pp. 851-855 1546-198X.

20. Eldawud, R., T. A. Stueckle, S. Manivannan, H. Elbaz, M. Chen, Y. Rojanasakul, and C. Z. Dinu, Real-time analysis of the effects of toxic, therapeutic and sub-therapeutic 
concentrations of digitoxin on lung cancer cells. Biosensors and Bioelectronics, 2014. 59: p. 192-199.

21. Ngamwongsatit, Puriya, Padmapriya P. Banada, Watanalai Panbangred, and Arun K. Bhunia, WST-1-based cell cytotoxicity assay as a substitute for MTT-based assay for rapid detection of toxigenic Bacillus species using $\mathrm{CHO}$ cell line. Journal of Microbiological Methods, 2008. 73(3): p. 211-215.

22. Pronk, Sander, Szilárd Páll, Roland Schulz, Per Larsson, Pär Bjelkmar, Rossen Apostolov, Michael R. Shirts, Jeremy C. Smith, Peter M. Kasson, David van der Spoel, Berk Hess, and Erik Lindahl, GROMACS 4.5: a high-throughput and highly parallel open source molecular simulation toolkit. Bioinformatics, 2013.

23. Marrink, Siewert J., H. Jelger Risselada, Serge Yefimov, D. Peter Tieleman, and Alex H. de Vries, The MARTINI Force Field: Coarse Grained Model for Biomolecular Simulations. The Journal of Physical Chemistry B, 2007. 111(27): p. 7812-7824.

24. van Meer, Gerrit, Dennis R. Voelker, and Gerald W. Feigenson, Membrane lipids: where they are and how they behave. Nat Rev Mol Cell Biol, 2008. 9(2): p. 112-124.

25. Berendsen, H. J. C., J. P. M. Postma, W. F. van Gunsteren, A. DiNola, and J. R. Haak, Molecular dynamics with coupling to an external bath. The Journal of Chemical Physics, 1984. 81(8): p. 3684-3690.

26. Kumar, Shankar, John M. Rosenberg, Djamal Bouzida, Robert H. Swendsen, and Peter A. Kollman, THE weighted histogram analysis method for free-energy calculations on 
biomolecules. I. The method. Journal of Computational Chemistry, 1992. 13(8): p. 10111021.

27. Bujacz, Anna, Structures of bovine, equine and leporine serum albumin. Acta Crystallographica Section D, 2012. 68(10): p. 1278-1289.

28. Kulakova, I. I., Surface chemistry of nanodiamonds. Physics of the Solid State, 2004. 46(4): p. 636-643.

29. Kubicki, J. D., L. M. Schroeter, M. J. Itoh, B. N. Nguyen, and S. E. Apitz, Attenuated total reflectance Fourier-transform infrared spectroscopy of carboxylic acids adsorbed onto mineral surfaces. Geochimica et Cosmochimica Acta, 1999. 63(18): p. 2709-2725.

30. Krüger, Anke, Carbon Materials and Nanotechnology2010, Weinheim: Wiley-VCH.

31. Greiner, N. R., Phillips, D. S., Johnson, J. D., Volk, F., Diamonds in detonation soot. Nature, 1988. 333: p. 440.

32. Patil, Swanand, Amanda Sandberg, Eric Heckert, William Self, and Sudipta Seal, Protein adsorption and cellular uptake of cerium oxide nanoparticles as a function of zeta potential. Biomaterials, 2007. 28(31): p. 4600-4607.

33. He, Chunbai, Yiping Hu, Lichen Yin, Cui Tang, and Chunhua Yin, Effects of particle size and surface charge on cellular uptake and biodistribution of polymeric nanoparticles. Biomaterials, 2010. 31(13): p. 3657-3666. 
34. Boitano, Anthony, Jonathan A. Ellman, Gary D. Glick, and Anthony W. Opipari, The Proapoptotic Benzodiazepine Bz-423 Affects the Growth and Survival of Malignant B Cells. Cancer Research, 2003. 63(20): p. 6870-6876.

35. Lin, Chung-Lun, Cheng-Huang Lin, Huan-Cheng Chang, and Meng-Chih Su, Protein Attachment on Nanodiamonds. The Journal of Physical Chemistry A, 2015. 119(28): p. 7704-7711.

36. Park, Eun-Jung, Jongheop Yi, Kyu-Hyuck Chung, Doug-Young Ryu, Jinhee Choi, and Kwangsik Park, Oxidative stress and apoptosis induced by titanium dioxide nanoparticles in cultured BEAS-2B cells. Toxicology Letters, 2008. 180(3): p. 222-229.

37. Kim, Ha Ryong, Mi Jie Kim, Soo Yeun Lee, Seung Min Oh, and Kyu Hyuck Chung, Genotoxic effects of silver nanoparticles stimulated by oxidative stress in human normal bronchial epithelial (BEAS-2B) cells. Mutation Research/Genetic Toxicology and Environmental Mutagenesis, 2011. 726(2): p. 129-135.

38. Wegener, J., C. R. Keese, and I. Giaever, Electric cell-substrate impedance sensing (ECIS) as a noninvasive means to monitor the kinetics of cell spreading to artificial surfaces. Exp Cell Res, 2000. 259(1): p. 158-66.

39. Siddiquei, H. R., A. N. Nordin, M. I. Ibrahimy, M. A. Arifin, N. H. Sulong, M. Mel, and I. Voiculescu, Electrical cell-substrate impedance sensing (ECIS) based biosensor for characterization of DF-1 cells. 2010: p. 1-4.

40. Kataoka, N., K. Iwaki, S. Mochizuki, Y. Ogasawara, K. Tsujioka, and F. Kajiya. Endothelial cell micro-motion monitored by ECIS during monocytes application. in 
[Engineering in Medicine and Biology, 1999. 21st Annual Conference and the 1999 Annual Fall Meetring of the Biomedical Engineering Society] BMES/EMBS Conference, 1999. Proceedings of the First Joint. 1999.

41. Chen, Szi-Wen, Jen Ming Yang, Jhe-Hao Yang, Shu Jyuan Yang, and Jong-Shyan Wang, A computational modeling and analysis in cell biological dynamics using electric cellsubstrate impedance sensing (ECIS). Biosensors and Bioelectronics, 2012. 33(1): p. 196203.

42. Müller, Jakob, Christian Thirion, and Michael W. Pfaffl, Electric cell-substrate impedance sensing (ECIS) based real-time measurement of titer dependent cytotoxicity induced by adenoviral vectors in an IPI-2I cell culture model. Biosensors and Bioelectronics, 2011. 26(5): p. 2000-2005.

43. Eldawud, Reem, Alixandra Wagner, Chenbo Dong, Yon Rojansakul, and Cerasela Zoica Dinu, Electronic platform for real-time multi-parametric analysis of cellular behavior post exposure to single-walled carbon Nanotubes. Biosensors and Bioelectronics, (0).

44. Goda, N., Y. Yamamoto, N. Kataoka, T. Nakamura, T. Kusuhara, S. Mohri, K. Naruse, and F. Kajiya, Quantitative evaluation of nano-order micromotion of cultured cells using electric cell-substrate impedance sensing method, in 13th International Conference on Electrical Bioimpedance and the 8th Conference on Electrical Impedance Tomography, $\mathrm{H}$. Scharfetter and R. Merwa, Editors. 2007, Springer Berlin Heidelberg. p. 122-125.

45. Shi, Yigong and Joan Massagué, Mechanisms of TGF- $\beta$ Signaling from Cell Membrane to the Nucleus. Cell, 2003. 113(6): p. 685-700. 
46. Assoian, R. K., Anchorage-dependent cell cycle progression. J Cell Biol, 1997. 136(1): p. $1-4$.

47. Rangaswami, H, A Bulbule, and GC Kundu, Osteopontin: role in cell signaling and cancer progression. Trends Cell Biol, 2006. 16: p. 79 - 87.

48. Mengesha, A. E. and B. B. C. Youan, 8 - Nanodiamonds for drug delivery systems, in Diamond-Based Materials for Biomedical Applications, R. Narayan, Editor 2013, Woodhead Publishing. p. 186-205.

49. Perevedentseva, E., N. Melnik, C.-Y. Tsai, Y.-C. Lin, M. Kazaryan, and C.-L. Cheng, Effect of surface adsorbed proteins on the photoluminescence of nanodiamond. Journal of Applied Physics, 2011. 109(3): p. 034704.

50. Campbell, Alan S., Chenbo Dong, Fanke Meng, Jeremy Hardinger, Gabriela Perhinschi, Nianqiang Wu, and Cerasela Zoica Dinu, Enzyme Catalytic Efficiency: A Function of BioNano Interface Reactions. ACS Applied Materials \& Interfaces, 2014. 6(8): p. 5393-5403.

51. Jiang, Jingkun, Günter Oberdörster, and Pratim Biswas, Characterization of size, surface charge, and agglomeration state of nanoparticle dispersions for toxicological studies. Journal of Nanoparticle Research, 2009. 11(1): p. 77-89.

52. Weng, Mao-Feng, Bo-Jui Chang, Su-Yu Chiang, Niann-Shiah Wang, and Huan Niu, Cellular uptake and phototoxicity of surface-modified fluorescent nanodiamonds. Diamond and Related Materials, 2012. 22(0): p. 96-104. 
53. Schrand, Amanda M., Jonathan B. Lin, Suzanne Ciftan Hens, and Saber M. Hussain, Temporal and mechanistic tracking of cellular uptake dynamics with novel surface fluorophore-bound nanodiamonds. Nanoscale, 2011. 3(2): p. 435-445.

54. Zhang, Bailin, Yingqi Li, Chia-Yi Fang, Cheng-Chun Chang, Chao-Sheng Chen, Yi-Ying Chen, and Huan-Cheng Chang, Receptor-Mediated Cellular Uptake of Folate-Conjugated Fluorescent Nanodiamonds: A Combined Ensemble and Single-Particle Study. Small, 2009. 5(23): p. 2716-2721.

55. Liu, Kuang-Kai, Chi-Ching Wang, Chia-Liang Cheng, and Jui- I. Chao, Endocytic carboxylated nanodiamond for the labeling and tracking of cell division and differentiation in cancer and stem cells. Biomaterials, 2009. 30(26): p. 4249-4259.

56. Chow, Edward K., Xue-Qing Zhang, Mark Chen, Robert Lam, Erik Robinson, Houjin Huang, Daniel Schaffer, Eiji Osawa, Andrei Goga, and Dean Ho, Nanodiamond Therapeutic Delivery Agents Mediate Enhanced Chemoresistant Tumor Treatment. Science Translational Medicine, 2011. 3(73): p. 73ra21-73ra21.

57. Merkel, Timothy J. and Joseph M. DeSimone, Dodging Drug-Resistant Cancer with Diamonds. Science Translational Medicine, 2011. 3(73): p. 73ps8-73ps8.

58. Snyder, Ryan J., Salik Hussain, Annette B. Rice, and Stavros Garantziotis, Multiwalled carbon nanotubes induce altered morphology and loss of barrier function in human bronchial epithelium at noncytotoxic doses. International Journal of Nanomedicine, 2014. 9: p. 4093-4105. 
59. Lin, J., H. Zhang, Z. Chen, and Y. Zheng, Penetration of lipid membranes by gold nanoparticles: insights into cellular uptake, cytotoxicity, and their relationship. ACS Nano, 2010. 4(9): p. 5421-9.

60. Verma, Ayush, Oktay Uzun, Yuhua Hu, Ying Hu, Hee-Sun Han, Nicki Watson, Suelin Chen, Darrell J. Irvine, and Francesco Stellacci, Surface-structure-regulated cellmembrane penetration by monolayer-protected nanoparticles. Nat Mater, 2008. 7(7): p. 588-595. 


\title{
Chapter 3
}

\section{Real Time Analysis of the Effects of Toxic, Therapeutic and Sub-therapeutic Concentrations of Digitoxin}

\begin{abstract}
Digitoxin belongs to a naturally occurring class of cardiac glycosides (CG); digitoxin is clinically approved for heart failure and known for its anti-cancer effects against non-small lung cancer cells (NSCLC). However, concerns associated with its narrow therapeutic index and its concentration-dependent mechanism of action are rising. Thus, before digitoxin implementation in designing and developing safer and more effective CG-based anti-cancer therapies, its pharmacological and safety profiles need to be fully elucidated. In this research we used a combinatorial approach to evaluate the anti-cancer mechanisms of digitoxin in real-time. Our approach employed a non-invasive electric cell impedance sensing technique as a proxy to monitor NSCLC behavior post-exposure to toxic, therapeutic and sub-therapeutic concentrations of the drug. By developing structure-function combinatorial relations we showed that digitoxin targets cancer cells in a time and dose-dependant manner by activating proapoptotic and anti-proliferative signaling cascades that results in strengthening cellular adhesion and sequestration of key regulatory proliferation protein from the nucleus.
\end{abstract}




\section{Introduction}

Digitoxin is a natural occurring cardiac glycoside (CG) [1] with a prolonged half-life, a well-established clinical profile, a narrow therapeutic window [1], and increased ability to readily cross both the blood brain and the placental barriers [2]. Laboratory investigations suggested that digitoxin exhibits high selectivity towards cancer cells when compared to healthy cells $[1,3]$ making the drug a viable chemotherapeutic alternative against several types of cancer from leukemia, to pancreatic and lung cancers $[4,5]$.

The anti-cancer mechanisms induced by digitoxin have been extensively studied and are mainly associated with the drug's ability to manipulate intracellular ion homeostasis which led to a downstream signaling cascade eventually inducing apoptosis and cell cycle arrest [6]. In particular, in vitro studies showed that exposure of non small lung cells, renal, pancreatic and breast cells to micromolar concentrations of digitoxin $(0.5-5 \mu \mathrm{M})$ inhibits $\mathrm{Na}^{+} / \mathrm{K}^{+}$-ATPase pump activity $[4,7]$, induces calcium-dependent activation of caspases and other hydrolytic enzymes $[1,8]$, causes generation of reactive oxygen species [9], activates the cell-cycle inhibitor p21Cip1 [9], directs the inhibition of topoisomerase activity and hypoxia-inducible factorla synthesis [10], and ultimately reduces viability and cell proliferation $[1,11]$. Complementary, cellular exposure to nanomolar concentrations of digitoxin $(10-100 \mathrm{nM})$ leads to inhibition of (HIF-1) and topoisomerase II synthesis [9], activation of phospholipase C [1, 12], phosphatidylinositol-3Kinase (PI3K) [12], tyrosine kinase (Src) [1, 13], and mitogen-activated protein kinase (MAPK) [9], affects cell cycle and anoikis [14] inducing alternations in membrane fluidity [15, 16], ultimately leading to cell apoptosis [17]. However, the nature of these in vitro studies only allowed for discrete time points monitoring and limited analysis of the cellular functions upon exposure, all after invasive or destructive preparation of the samples, and labor intensive and time consuming 
analysis. Therefore, further studies are needed to better characterize the pharmacological and safety profiles of digitoxin before its chemotherapeutic implementation, especially considering that therapeutic concentrations of digitoxin varies according to the age and weight of the patient, generally ranging from 26-46nM [18]. Such studies do not only have to account for the narrow therapeutic window or drug's dose-dependent and selective apoptosis of cancer cells [7, 15], but also need to allow monitoring of the cellular systems without lag time between sample collection and data analysis.

Electric cell-substrate impedance sensing (ECIS) is a non-invasive and quantitative form of cell-based sensing that utilizes identical small gold-film electrodes deposited on the bottom of cell culture dishes to measure the cellular resistance and its alteration upon changes in cell morphology, spreading, attachment and migration $[19,20]$, all as a function of applied frequencies and in real-time [21, 22]. The applicability of ECIS was extended to inhibition assays for cytochalasin-D (cytoskeletal inhibitor) [23], prostaglandin E2 (inflammatory mediator) [24], bacterial protease [25], or platelet-activating factors that affect in cellular adhesion [26].

By combining standard in vitro cell viability and cell-based cytotoxicity assays with ECIS, we aim to investigate the anti-proliferative and pro-apoptotic mechanisms of non-small lung cancer cells (NSCLC) exposed to different concentrations of digitoxin in real-time. NCI-H460 cells were chosen as model NSCLC based on their sensitivity to digitoxin $[4,27]$ and increased resistance to chemotherapy $[28,29]$. We hypothesized that NCI-H460 exposure to toxic $(80 \mathrm{nM})$, therapeutic $(40 \mathrm{nM})$ and sub-therapeutic $(25$ and $10 \mathrm{nM})$ concentrations of digitoxin is associated with changes in the cellular viability that can be monitored using an individual cell as a primary transducer and recording its real-time alterations in morphology and adhesion profile. Further, based on the key role of the cyclin-dependent kinase-4 (CDK4) in regulation of cell proliferation 
and cell cycle $[30,31]$, we hypothesized that exposure to digitoxin targets cellular adhesion pathways in a dose and time dependent manner by sequestering CDK4 in the cytoplasm and thus reducing its nuclear levels. Further, CDK4 association with viable candidates responsible for cellular junctions formation can be monitored in real-time as a change in the cellular attachment profile. Our findings underscore the potential of digitoxin to be used as the next generation of chemotherapeutic drugs that target cell adhesion and cell cycle profiles for improved anti-cancer activity.

\section{Material and Methods}

\section{Cell culture and treatment}

Human lung cancer epithelial cells (NCI-H460; ATCC, VA) were cultured in Roswell Park Memorial Institute-1640 medium (RPMI-1640; Sigma Chemicals, MO) supplemented with 10\% fetal bovine serum (FBS; Atlanta Biologicals,GA), $2 \mathrm{mM} \mathrm{L-glutamine} \mathrm{and} \mathrm{100-units/ml}$ penicillin/streptomycin (Sigma Chemicals, MO) and maintained in a humidified atmosphere at $37^{\circ} \mathrm{C}$ under $5 \% \mathrm{CO}_{2}$. Cells were passaged regularly using $0.25 \%$ (w/v) trypsin (Molecular Probles, OR) with $1.5 \mathrm{mM}$ ethylene diaminetetracetic acid (EDTA; Molecular Probes, OR). Stock concentrations of digitoxin (Sigma Chemicals, MO) were made in dimethyl sulfoxide (DMSO; Sigma Chemicals, MO) and diluted to 1000x exposure concentrations as previously described [6]. Digitoxin exposure was performed in a medium containing 1\% FBS, 2 mM L-glutamine and 100units/ml penicillin/streptomycin. The concentration of FBS was reduced due to existing concerns regarding digitoxin's interaction with serum proteins [32] and to better approximate the minimum concentrations and times that were required to achieve drugs' activity in vitro [6].

\section{Apoptosis assay}


Cells were seeded overnight in 12-well plates (Fisher, PA) at $2 \times 10^{5}$ cell $/ \mathrm{mL}$ and treated with $0,10,25,40$ and $80 \mathrm{nM}$ digitoxin for 24,48 and $72 \mathrm{~h}$ respectively. After treatment, the cells were incubated with $10 \mu \mathrm{g} / \mathrm{ml}$ Hoechst-33342 (Molecular Probes, OR) for $30 \mathrm{~min}$. The percentage of cells having intensely condensed chromatin and/or fragmented nuclei was scored using fluorescence microscope (Leica Microsystems, IL). Approximately 1,000 nuclei from ten random fields were analyzed for each sample. The apoptotic percentage was calculated as the percentage of cells with apoptotic nuclei over the total number of cells per field of view.

\section{Western blot analysis}

Cells were seeded overnight in 6 -well plates at a density of $6 \times 10^{5}$ cell/well, and treated with 0,10 or $25 \mathrm{nM}$ digitoxin in $0.1 \%$ DMSO for $24 \mathrm{~h}$. Subsequently, cells were placed on ice and lysed for $30 \mathrm{~min}$ in a lysis buffer containing $2 \%$ Triton X-100, 1\% sodium dodecyle sulfate (SDS), $100 \mathrm{mM}$ Sodium Chloride ( $\mathrm{NaCl}), 10 \mathrm{mM}$ Tris-Hydrochloric acid ( $\mathrm{HCl})$, Complete Mini cocktail protease inhibitors (all reagents are purchased from Roche, IN) and 1 mM EDTA insoluble cellular debris was pelleted by centrifugation at $4^{\circ} \mathrm{C}$ and $16,000 \mathrm{~g}$. The supernatant was collected and used to determine the total protein content using standard Bicinchoninnic acid assay (BCA, Thermo Scientific, IL). Briefly, working reagent was prepared according to the manufacturer instructions by mixing 50 parts of reagent A with 1 part of reagent B (reagents included with kit). Two $\mu \mathrm{L}$ of each sample was added to a 96-well plate and incubated with $200 \mu \mathrm{L}$ of the working reagent at $37{ }^{\circ} \mathrm{C}$ for $30 \mathrm{~min}$; experiments were performed in duplicate. Control calibration curve was prepared using serial dilutions of standard bovine serum albumin (BSA). Absorbance at 562nm was recorded on a BioTek 96-plate reader (BioTek, Winooski, VT).

The supernatant was separated by a 10\% SDS-PAGE gel and transferred to polyvinylidene 
fluoride (PVDF) membranes using the iBlot $^{\circledR}$ Dry Blotting System (Invitrogen, CA). Membranes were blocked in 5\% skim milk in Tris-buffered saline (TBST, $25 \mathrm{mM}$ Tris- $\mathrm{HCl}, 125 \mathrm{mM} \mathrm{NaCl}$, and $0.1 \%$ Tween-20; Sigma Chemicals,MO) for $1 \mathrm{~h}$ at room temperature, and subsequently incubated with anti-CDK4 primary antibody (Cell Signaling, MA) at $4{ }^{\circ} \mathrm{C}$ overnight. The membranes were subsequently washed three times in phosphate buffer saline (PBS; Lonza, MD) containing $1 \%$ Tween-20 for $10 \mathrm{~min}$ each, incubated with horseradish peroxidase-conjugated secondary antibody (Cell Signaling, MA) for $1 \mathrm{~h}$ at room temperature, then washed again for three more times each for $10 \mathrm{~min}$ in TBST. Finally, the samples were analyzed by chemiluminescence (Supersignal West Pico; IL). Band quantification via densitometry was performed using ImageJ software, version 10.2.

\section{Trypan-blue exclusion assay}

NCI-H460 cells were seeded overnight in 12 -well plates at a density of $2 \times 10^{5} \mathrm{cell} / \mathrm{mL}$, and treated with $0,10,25,40$ or $80 \mathrm{nM}$ digitoxin for 24,48 , and $72 \mathrm{~h}$ respectively. Cells were subsequently washed with PBS, trypsinized $(0.25 \%)$, suspended in $10 \%$ media and stained with $0.4 \%$ trypan-blue (Invitrogen, CA) at 1:1 volume ratio and analyzed using Countess automated cell counter (Invitrogen, CA).

\section{Electric cell-substrate impedance sensing (ECIS)}

Real-time quantification of cellular behavior was conducted using an electric cell impedance sensing instrument (ECIS-Z $\Theta$, Applied Biophysics, NY). In one set of experiments, two ECIS arrays (8W10E+), each containing 8-wells with 40 gold electrodes, were simultaneously employed to provide concomitant measurements of 16 samples at multiple frequencies. Prior to any experiment, the gold electrodes were stabilized for $3 \mathrm{~h}$ in $400 \mu 1$ RPMI media to account for 
electrode variances and to create a reference line associated with free electrodes; subsequently, the array holder was placed in a humidified incubator at $37{ }^{\circ} \mathrm{C}$ and $5 \% \mathrm{CO}_{2}$ to provide optimal conditions for cellular growth. NCI-H460 cells were added at a density of $2 \times 10^{5}$ cell/mL in a volume of $400 \mu \mathrm{l} /$ well. Cells were allowed to settle and grow over the gold electrodes and form a confluent monolayer for $24 \mathrm{~h}$. The formation of the cellular monolayer was indicated as a settlement in the resistance value with minor fluctuation caused by cellular micromotion $[19,22$, 33]. Upon monolayer formation cells were treated with $0,10,25,40$ or $80 \mathrm{nM}$ digitoxin and their cellular behavior was monitored for $48 \mathrm{~h}$ post-exposure.

\section{Statistical analysis}

Results are presented as mean \pm standard deviation. Experiments (viability, apoptosis and Western blot) were performed in duplicates and repeated at least 3 times. ECIS experiments were performed in duplicates and repeated at least four times, for a total of minimum 8 replicates per dose. Changes in the behavior of the cells (i.e., resistance) were recorded every $180 \mathrm{sec}$ for the duration of the experiments with each time point being an average of 16 replicates ( 2 arrays with 8 wells each). Two-way analysis of variance and unpaired two-tailed Student's t-test were performed using JMP 8.0 (SAS Institute) and SigmaPlot 10.0 (Systat Software Inc.). Results were considered significant for $\mathrm{p}^{*}<0.05$. 


\section{Results}

\section{Digitoxin induced apoptosis in a time and dose-dependent manner}

To evaluate the effects of digitoxin on cellular apoptosis, confluent monolayers of human lung cancer (NCI-H460) cells were exposed to toxic, therapeutic and sub-therapeutic concentrations of digitoxin for 24,48 and $72 \mathrm{~h}$ respectively. Visual inspection of the $24 \mathrm{~h}$ exposed cells showed minor differences in their nuclear morphology for the cells treated with $10 \mathrm{nM}(5 \%)$ and $25 \mathrm{nM}(15 \%)$ digitoxin when compared to the untreated cells (Figure 1A). In contrast, the nuclei of the cells treated with 40 and $80 \mathrm{nM}$ digitoxin showed major changes $(\geq 50 \%)$ in their morphologies, i.e., rounding, swelling, condensation and fragmentation. Such changes are considered early indicators of cellular apoptosis $[34,35]$.

The percentage of apoptotic cells at 24, 48 and $72 \mathrm{~h}$ post-exposure to toxic, therapeutic and sub-therapeutic concentrations of digitoxin is shown in Figure 1B. Analysis of variance showed that the NCI-H460 apoptosis was both dose and time-dependent. In particular, $24 \mathrm{~h}$ after exposure, the cells treated with $10 \mathrm{nM}$ showed no significant changes in their apoptotic percentage; however, increased apoptosis was noticed after 48 and $72 \mathrm{~h}$ of digitoxin exposure. Cells exposed to 25, 40 and $80 \mathrm{nM}$ digitoxin exhibited higher apoptosis within first $24 \mathrm{~h}$ with a significant increase after 48 and 72 h respectively. Values for the IC50 (half-inhibition concentration) derivated using a three-parameter sigmoid regression analysis were $43.4 \mathrm{nM}$, to $36.6 \mathrm{nM}$ and $31.6 \mathrm{nM}$ at 24,48 and $72 \mathrm{~h}$ post-exposure respectively. 
(a)
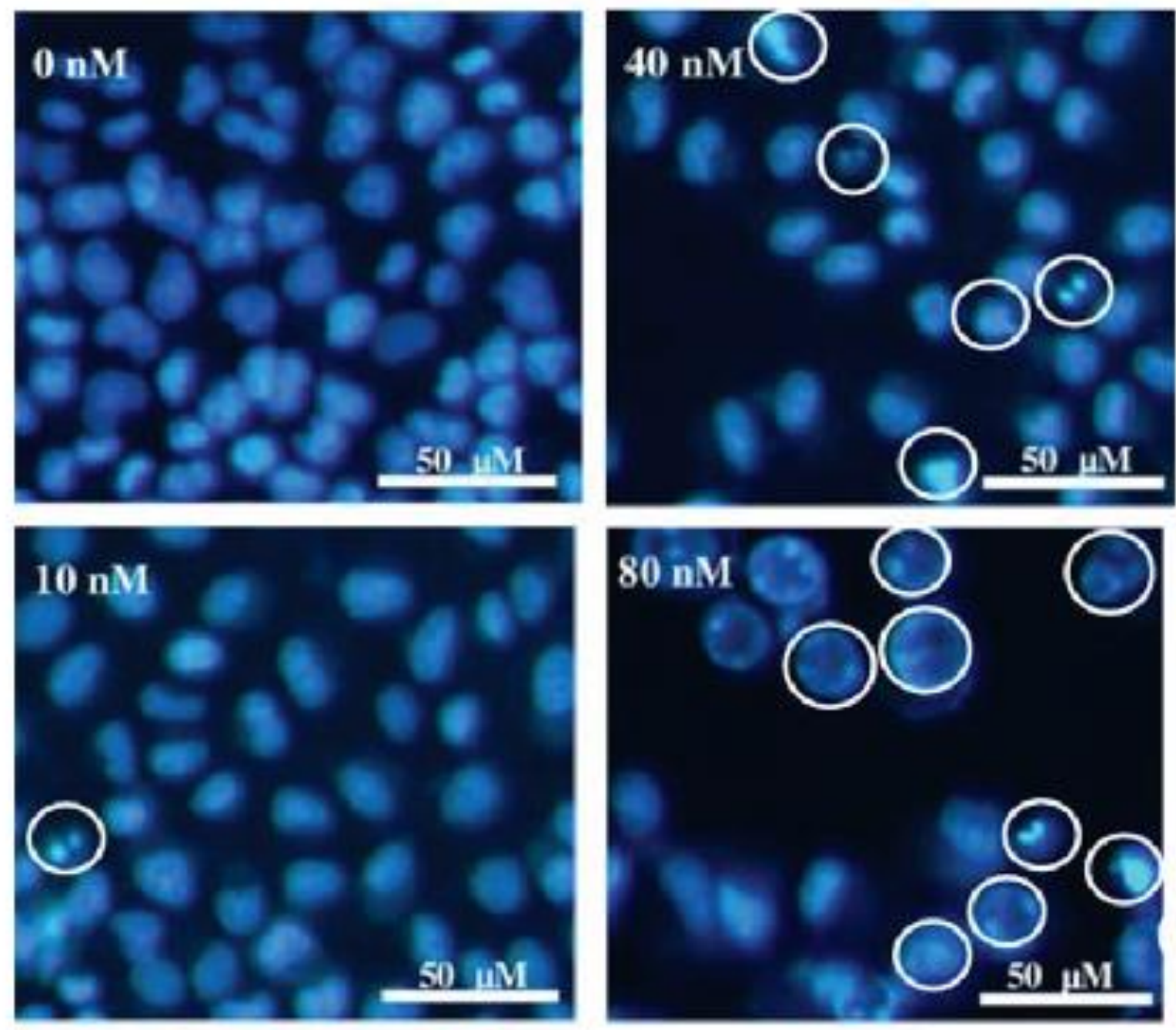

\section{$25 \mathrm{nM}$}
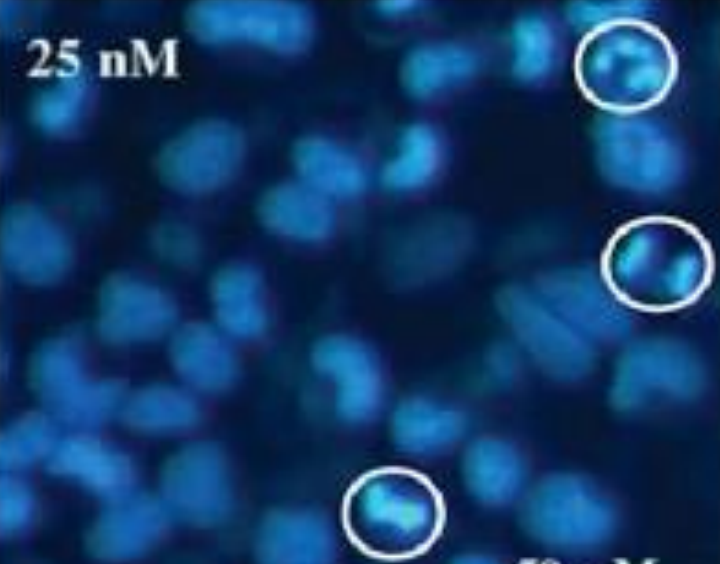

$50 \mathrm{nM}$ 
(b)

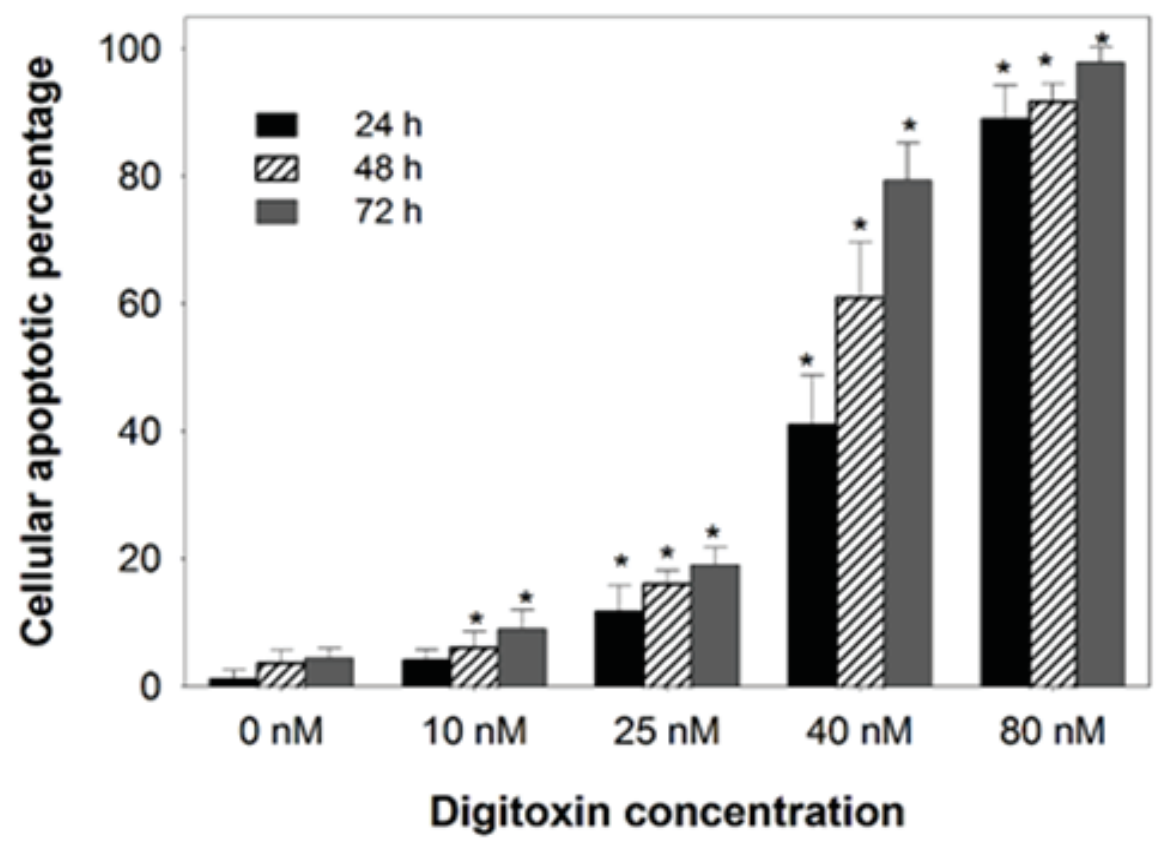

Figure 1: (a) Fluorescent images of Hoechst stained NCI-H460 cells $24 \mathrm{~h}$ post-exposure to digitoxin. Cells treated with 40 and $80 \mathrm{nM}$ digitoxin showed major changes in their nuclei morphologies (white circles) relative to controls or cells treated with 10 and $25 \mathrm{nM}$ digitoxin. (b) Percentage of apoptotic cells upon exposure to digitoxin is dose and time-dependent. Difference is considered significant for $* p<0.05$.

\section{Digitoxin inhibited cellular proliferation}

To study the effects of toxic, therapeutic and sub-therapeutic concentrations of digitoxin on the NCI-H460 cell proliferation, a live cell exclusion assay was performed. The results showed that exposure to digitoxin caused inhibition of cellular proliferation in a dose and time-dependent manner (Figure 2A). Specifically, no differences relative to controls were noticed $24 \mathrm{~h}$ post- 
exposure the cells treated with $10 \mathrm{nM}$ digitoxin; however, significant inhibitory effects were observed after $48(18 \%)$ and $72 \mathrm{~h}(27 \%)$ respectively. For the cells treated with 25,40 and $80 \mathrm{nM}$ digitoxin, the decrease in the live cell counts was significant within the first $24 \mathrm{~h}$ with $35 \%, 54 \%$ and $82 \%$ respectively. This subsequently increased to $43 \%, 70 \%$ and $99 \%$ after $48 \mathrm{~h}$ exposure and $70 \%, 85 \%$ and $100 \%$ after $72 \mathrm{~h}$ respectively.

Since inhibition of cell proliferation was the major effect $24 \mathrm{~h}$ post-exposure to subtherapeutic concentrations of digitoxin, we also examined the expression of cyclin-dependent kinases-4 (CDK4), a key regulatory protein that controls cellular proliferation and cell cycle progression at the G1/S phase $[30,31]$. Western blot analysis showed significant decrease in the expression of CDK4 after exposure to both 10 and $25 \mathrm{nM}$, with a $35 \%$ and $60 \%$ reduction in the CDK4 expression relative to expression of control $\beta$-actin (Figure $2 \mathrm{~B}, \mathrm{C}$ ).

(a)

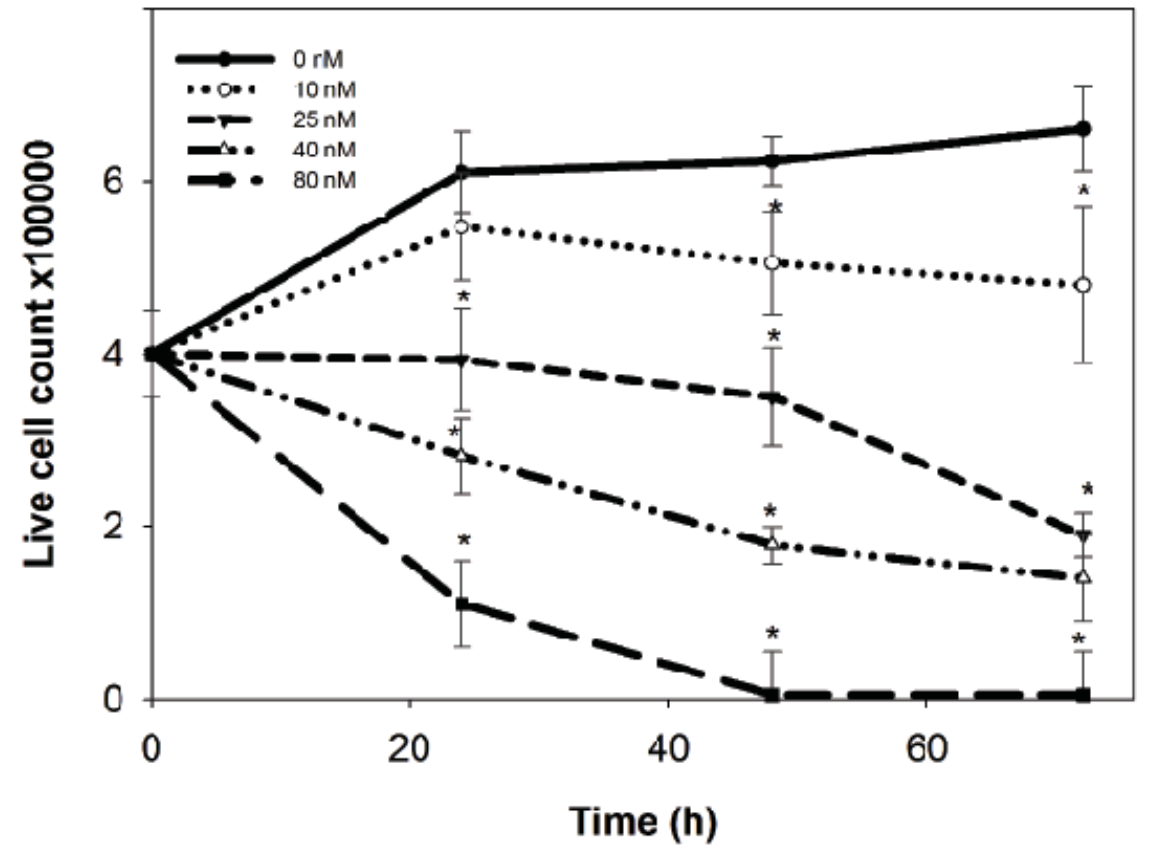


(b)

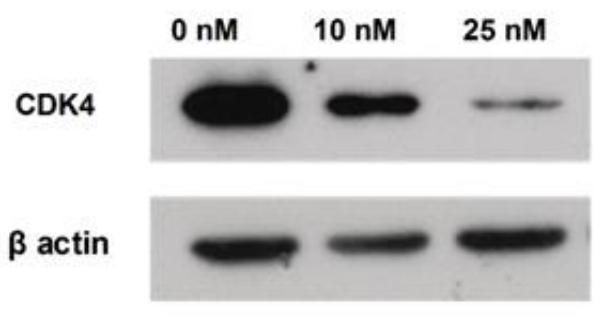

(c)

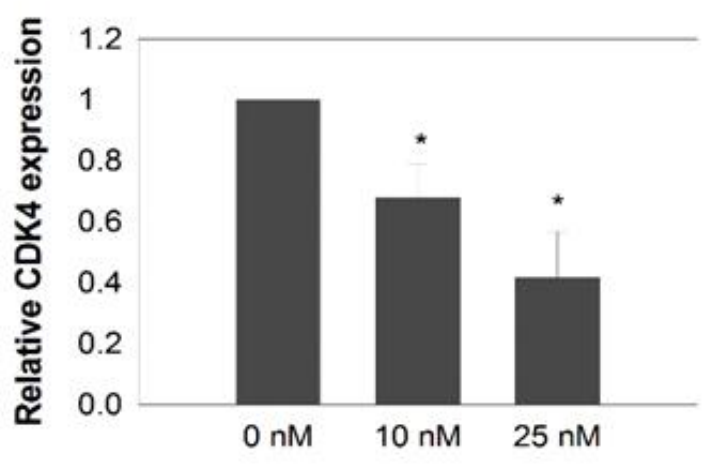

Digitoxin concentration

Figure 2: (a) Live cell counts after 24, 48 and $72 \mathrm{~h}$ exposure to different concentrations of digitoxin. (b) Western blot analysis of CDK4 expression relative to control $\beta$-actin $24 \mathrm{~h}$ postexposure to digitoxin. (c) Quantification of CDK4 expression levels relative to $\beta$-actin. A significant difference is indicated for $* p<0.05$.

\section{Real time cell monitoring using electric cell impedance sensing}

Real time analysis from the time of cell inoculation into the wells, to the formation of a confluent monolayer, and subsequently $48 \mathrm{~h}$ post-exposure to toxic, therapeutic and subtherapeutic concentrations of digitoxin were performed using electric cell impedance sensing (ECIS). Previous studies showed that cells immobilized onto gold electrodes have similar behaviors with their counterparts immobilized onto polystyrene surfaces [33, 36]. Figure 3A shows an eight well ECIS array with inter-finger-like arrangement and the current pathways contributing to the resistance measurements. Once the cells spread onto the gold electrodes, their insulating nature of the plasma membrane constricted the current flow in the spaces beneath the basal membrane and the electrode surface as well as in the para-cellular spaces between adjacent cells, 
leading to the recorded resistance (Giaever and Keese 1991, 1993). Alpha is a function of the distance between the basal membrane and cellular radius according to the following equation:

$$
\alpha=R_{c}(\rho / h)^{0.5}
$$

where $\rho$ represents the specific resistivity of the electrolyte (i.e., culture media) underneath the cells, $\mathrm{h}$ represents the distance at which the cells hover above the electrode, and $\mathrm{R}_{\mathrm{c}}$ is the cellular radius.

The changes in NCI-H460 resistance were mathematically fitted and are shown in Figure 3B. The monolayer resistance value was normalized to the base level of the cell free electrodes at $4 \mathrm{kHz}$ operational frequency; data was collected every $180 \mathrm{sec}$ (Supplementary information Figure S1). Region A reflects the changes in resistance from inoculation to the cells settling onto the electrodes and includes the lag period or the time required by the cells to overcome the disturbance caused by inoculation. This region was fitted using a linear computational model [37, 38]. In particular, for $\mathrm{t}<2 \mathrm{~h}$

$$
R(t)=a t+b
$$

where $\mathrm{R}(\mathrm{t})$ is the resistance value of the cells settled onto the electrodes, and $\mathrm{a}$ and $\mathrm{b}$ are parameters estimated using a least-square algorithm. Region B was directly associated with the cells ability to spread and form increased cell-cell contacts. The starting point for region B was determined by finding the theoretical point where the two mathematical models intercept (i.e., the linear model for region A and the exponential fit for region B). Specifically, for $\mathrm{t} \geq \tau$,

$$
R(t)=R_{1} e^{\mathrm{kt}}
$$

where $R_{1}$ and $k$ are parameters estimated by the least-square algorithm and $\tau$ is the lag period. Using a Taylor's series expansion of a first and second order, the lag period was found to be $1 \mathrm{~h} 44$ min and $1 \mathrm{~h} 46$ min respectively for the NCI-H460 cells. Upon complete coverage of the gold 
electrodes, cellular proliferation was hindered by contact inhibition shown in region $\mathrm{C}$ and modeled using a second order polynomial. Specifically,

$$
R(t)=a t^{2}+\mathrm{bt}+\mathrm{c}
$$

where $\mathrm{R}(\mathrm{t})$ is the resistance of cells the reaching the hindered growth and $\mathrm{a}, \mathrm{b}$ and $\mathrm{c}$ are parameters estimated using the least-square algorithm. Finally, upon the complete formation of cell monolayer, the normalized resistance reached a plateau, i.e. region D.

Cell attachment and monolayer formation was further confirmed by evaluating the changes in $\alpha[19,33,39]$ (Figure 3C). An increase in $\alpha$ indicates a decrease in the distance between the gold electrode and the cell basal membrane (h). The results showed that the changes in $\alpha$ followed a similar trend to the changes in the cellular resistance, with no fluctuations being observed after complete formation of the cell monolayer.

(a)

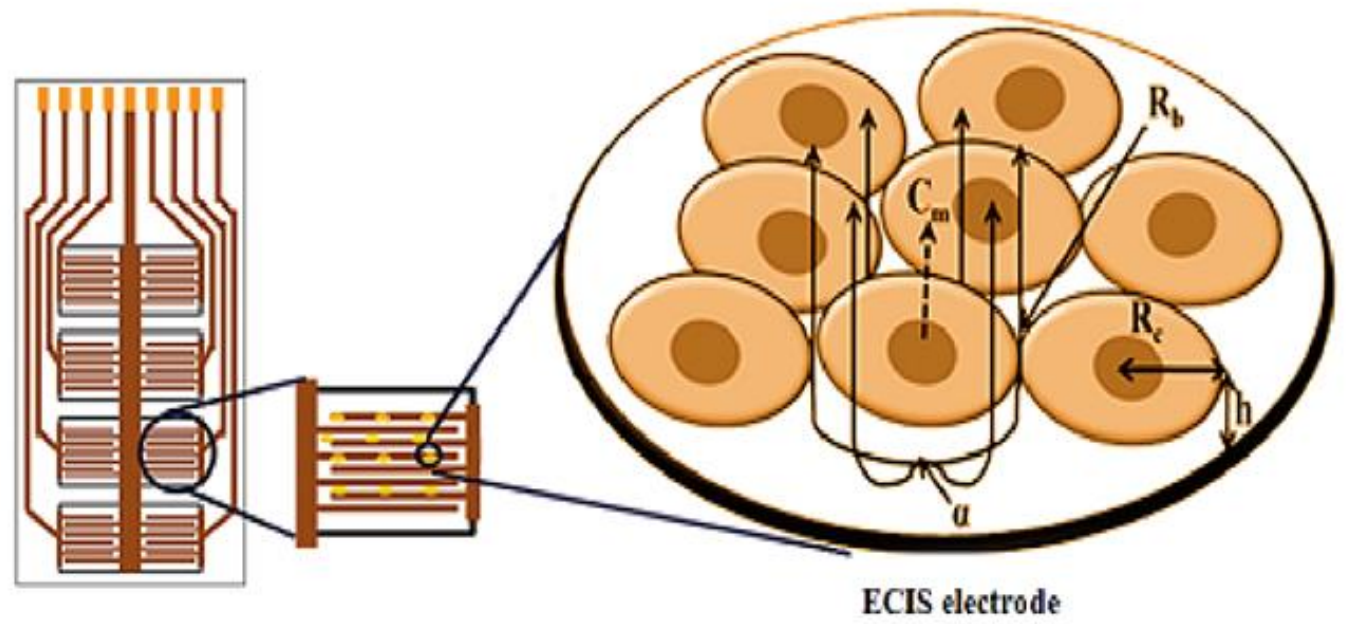


(b)

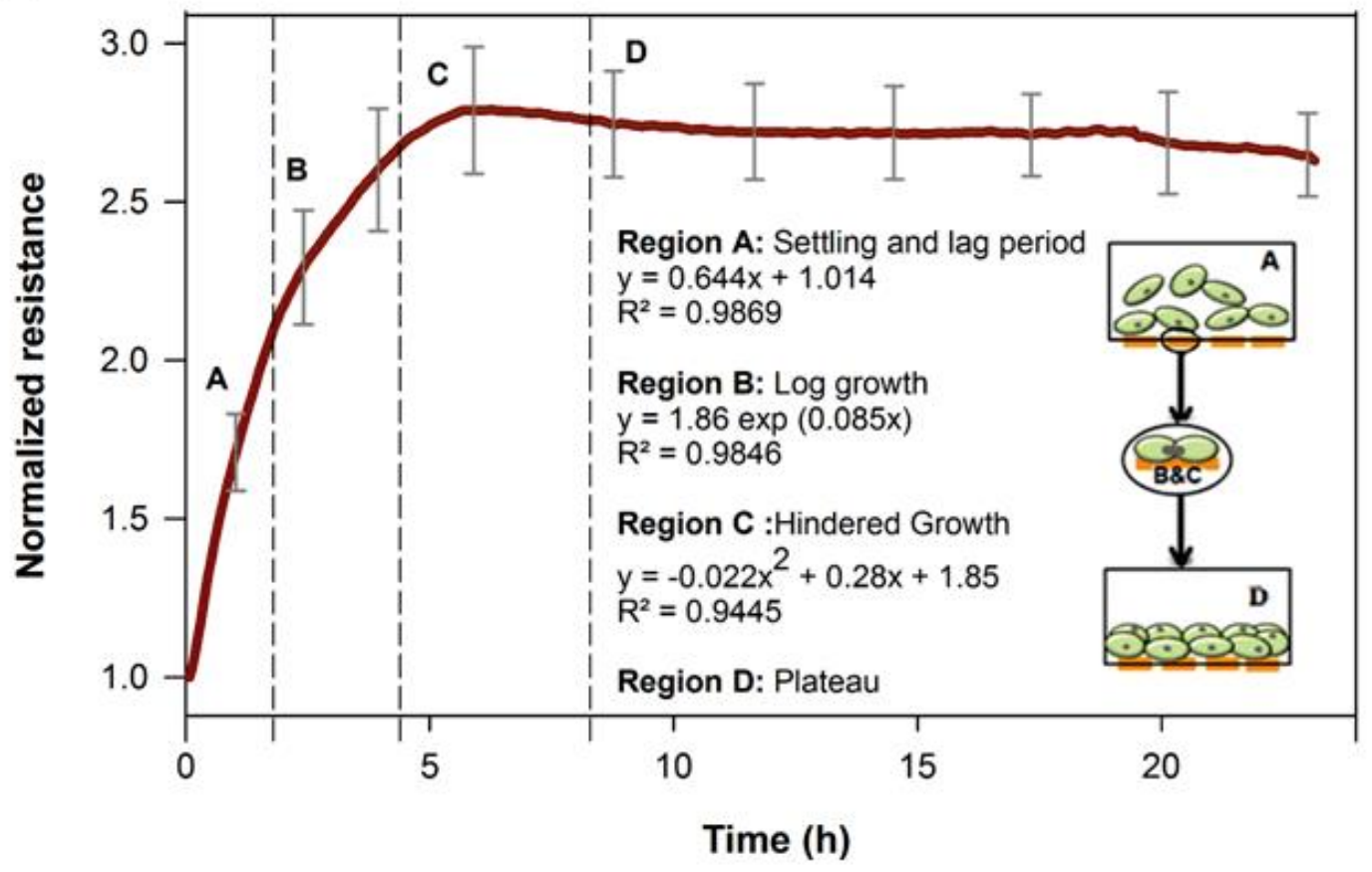

(c)

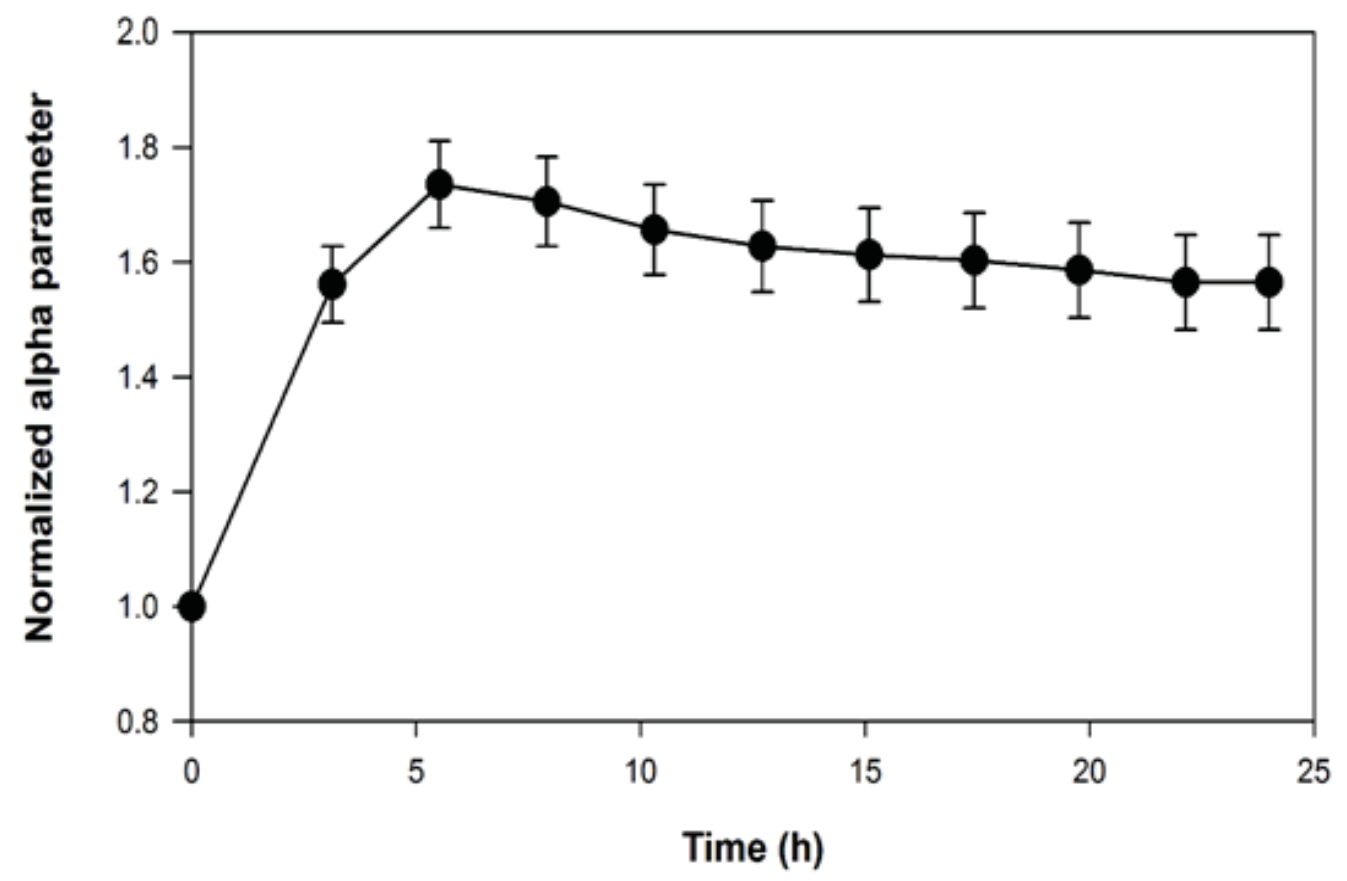

Figure 3: (a) Schematic illustration of an ECIS array, with a demonstration of the ECIS current pathways accounting for the resistance measurements. (b) Representative real-time measurements 
of the normalized resistance mathematically modeled according to the growth and spreading behavior of the NCI-H460 cells over the gold electrodes; the standard deviation is shown every two and a half hours and represents an average of 16 measurements ( 2 arrays with 8 wells each). (c) Real-time changes in $\alpha$ parameter.

Twenty-four hours post inoculation, the confluent monolayer was exposed to toxic, therapeutic and sub-therapeutic concentrations of digitoxin. Figure 4A shows the representative normalized resistance of control (untreated) and cells treated with different concentrations of digitoxin as measured every $3 \mathrm{~min}$ for a total of $48 \mathrm{~h}$. An initial increase in resistance with a slower rate for 10 and $25 \mathrm{nM}$ (sub-therapeutic) and a higher rate for both 40 (therapeutic) and $80 \mathrm{nM}$ (toxic) concentrations was recorded. Upon stabilization ( $\sim 5 \mathrm{~h}$ post-exposure), the resistance of the cells exposed to 10 and $25 \mathrm{nM}$ digitoxin showed higher absolute values than the resistance of the controls. Further, the resistance values of the cells treated with $25 \mathrm{nM}$ digitoxin had higher absolute values relative to the absolute values of the $10 \mathrm{nM}$ treated cells. Similarly, the resistance of the cells treated with 40 and $80 \mathrm{nM}$ digitoxin underwent drastic changes. Specifically, after an initial increase, a sharp drop was recorded at $5 \mathrm{~h}$ for the cells exposed to toxic concentrations of digitoxin (i.e., $80 \mathrm{nM}$ ).

A dramatic loss of cellular resistance was observed after about 10h of exposure and was indicative of an acute effect of digitoxin on the cell adhesion or membrane impedance. Complementary, after an initial increase in the resistance of the cells exposed to $40 \mathrm{nM}$ digitoxin, a gradual resistance drop was recorded starting at $8 \mathrm{~h}$ post-exposure. Further, $40 \mathrm{nM}$ treated cells showed a smaller change in the resistance rate when compared to $80 \mathrm{nM}$ digitoxin treated cells. 
To correlate the observed apoptotic percentage with the real time analysis of the resistance post-exposure to 40 and $80 \mathrm{nM}$ digitoxin, we investigated cellular attachment. Our results showed that cells treated with 40 and $80 \mathrm{nM}$ digitoxin exhibited a significant drop in $\alpha$ after 5 and $9 \mathrm{~h}$ of exposure respectively (Figure 4B). Our apoptotic data and previous experiments correlated such changes to the loss of cellular monolayer resulting from cell death and cell de-attachment $[19,40]$. Contrary, cells exposed to 10 and $25 \mathrm{nM}$ digitoxin showed a significant increase in $\alpha$ relative to controls, indicating a stimulation of their cellular attachment.

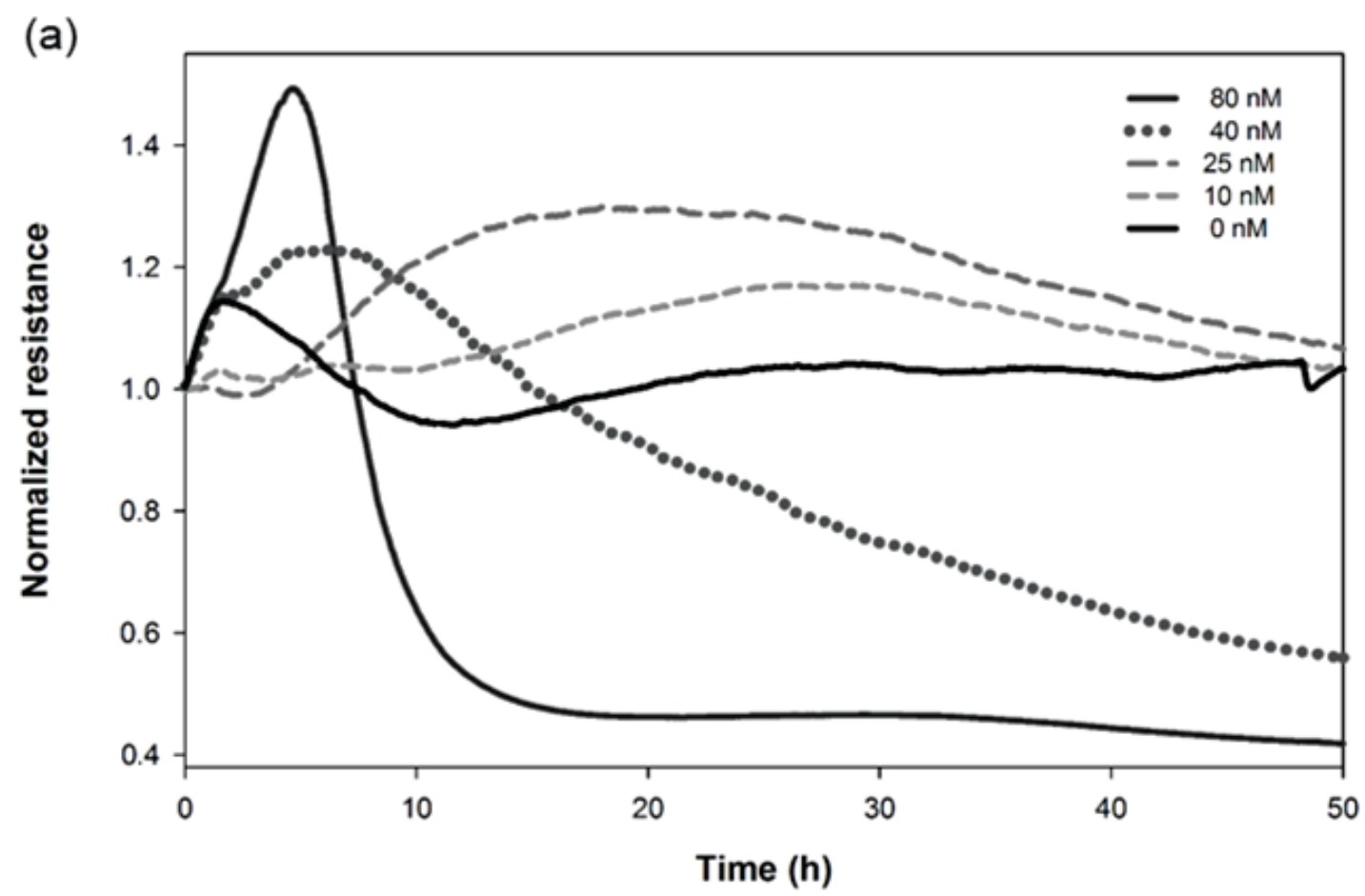


(b)

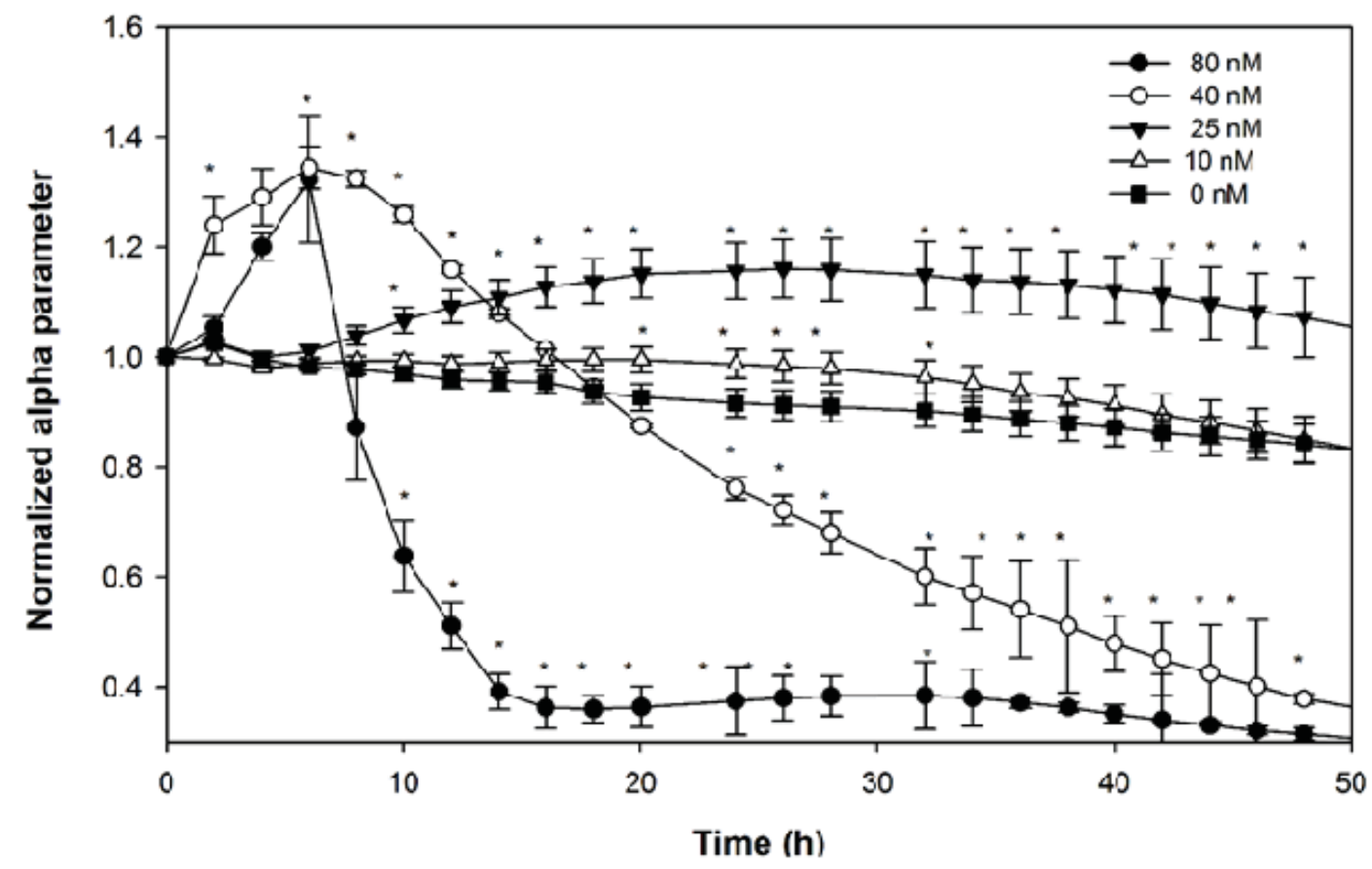

Figure 4: (a) Representative analysis of the real-time behavior of NCI-H460 cells following digitoxin exposure. (b) Changes in $\alpha$ parameter post-exposure to digitoxin in real-time; * signifies changes considered significant $p<0.05$ 


\section{Discussion}

We hypothesized that exposure to toxic, therapeutic and sub-therapeutic concentrations of digitoxin induces changes in NCI-H460 behavior that correlate with digitoxin's pharmacological profiles and can be analyzed in real time. By combining standard cell viability and cell-based cytotoxicity assays, we showed that the percentage of apoptotic cells was both dose and timedependent, with significant increases in the apoptotic percentage for the cells exposed to 25,40 and $80 \mathrm{nM}$ digitoxin and minor changes for the cells exposed to $10 \mathrm{nM}$ relative to controls after $24 \mathrm{~h}$ exposure. Further, our results showed a dose-dependent decrease in the expression of key cell cycle regulatory protein $\mathrm{CDK} 4$ relative to control $\beta$-actin.

By exploiting the naturally evolved sensitivity of the cells and by using the cell as a primary transducer, we recorded the changes in cellular behavior as real time changes in cellular resistance and adhesion post-exposure to toxic, therapeutic and sub-therapeutic concentrations of digitoxin $[19,37]$. Our dynamic combinatorial analysis showed predominantly pro-apoptotic and antiproliferative effects upon treatment with toxic and therapeutic concentrations of digitoxin (i.e., 80 and $40 \mathrm{nM}$ ) confirmed by a sharp drop in resistance. Complementary, the cells treated with subtherapeutic concentrations of digitoxin (i.e., 25 and $10 \mathrm{nM}$ ) showed a hindered proliferation rate and increased cellular adhesion. Changes in $\alpha$ were directly related to the restrictions in the AC current caused by changes in the distance between the basal membrane and the ECIS electrodes. Cellular detachment and reduction in $\alpha$ post-exposure to 80 and $40 \mathrm{nM}$ digitoxin were correlated to changes in nuclear morphology, cellular proliferation and cytotoxicity. Digitoxin's concentration-dependent changes illustrated the advantages of continuous monitoring provided by ECIS; in particular, the ECIS allowed for intermediate time events analysis identifying dramatic losses in cell resistance upon exposure to toxic concentrations of digitoxin and increases in the 
resistance of the cells exposed to sub-therapeutic concentrations. These differential cellular responses suggest the existence of threshold concentrations associated with either cellular mechanisms responsible for increased cellular adhesion or with a dose-dependent cellular inhibition coefficient profile of digitoxin.

Exposure to CGs is known to cause G1/S or G2 arrests in cancer cells at sub-cytotoxic levels [41, 42]. Binding of nuclear CDK4 to cyclin D regulates retinoblastoma tumor suppressor $(\mathrm{Rb})$ and E2F group expression that promotes $\mathrm{G} 1 / \mathrm{S}$ phase transition $[43,44]$. Thus, therapeutic targeting of CDK4 in cancer cells has been proposed as an effective approach for cancer therapy [45]. Based on the decrease in CDK4 expression and real-time increase in cellular resistance upon exposure to 10 and $25 \mathrm{nM}$ digitoxin, we hypothesize that digitoxin targets cellular adhesion by changing the subcellular distribution of CDK4 [30,31] (Figure 5). Our hypothesis is supported by previous studies that showed that reduction in the nuclear levels of CDK4 could result from CDK4 sequestration in the cytoplasm and CDK4 association with ZONAB [46]. ZONAB is a Y-box transcription factor whose localization and transcriptional activity is regulated by the tight junction-associated candidate tumor suppressor and scaffolding protein zona occluding 1 (ZO-1) $[47,48]$.

ZONAB binds to the Src homology 3 (SH3) binding domain of ZO1 at the cell periphery $[49,50]$ and helps regulate para-cellular permeability $[47,51]$ by its indirect participation in the formation of tight, adherent and gap junctions. ZO1 interaction with the cytoplasmic domain of occludins [52], junctional adhesion molecule [53] and claudins [54] regulates tight junction formation; additionally, ZO-1 interaction with E-cadherin [55] and nectine [51, 56] helps in the formation and maintenance of adherence junctions while ZO-1 interaction with gap junctions proteins $[47,57]$ regulates the formation of gap junctions. Given ZONAB's unaffected levels of 
expression upon cellular exposure to digitoxin (Supplementary information Figure S2), the propensity of ZONAB to bind to ZO1 and ZO-1's structural role in the basal podosomes biogenesis [48], it is also reasonable to assume that exposure to digitoxin leads to increased podosome formation that could enhance cell adhesion. Increasing ZO-1/ZONAB binding at the cell periphery, and subsequently the decrease in the nuclear levels of CDK4 levels would explain both the slow proliferation observed for the NCI-H460 cells exposed to $10 \mathrm{nM}$ digitoxin as well as their higher adhesion.

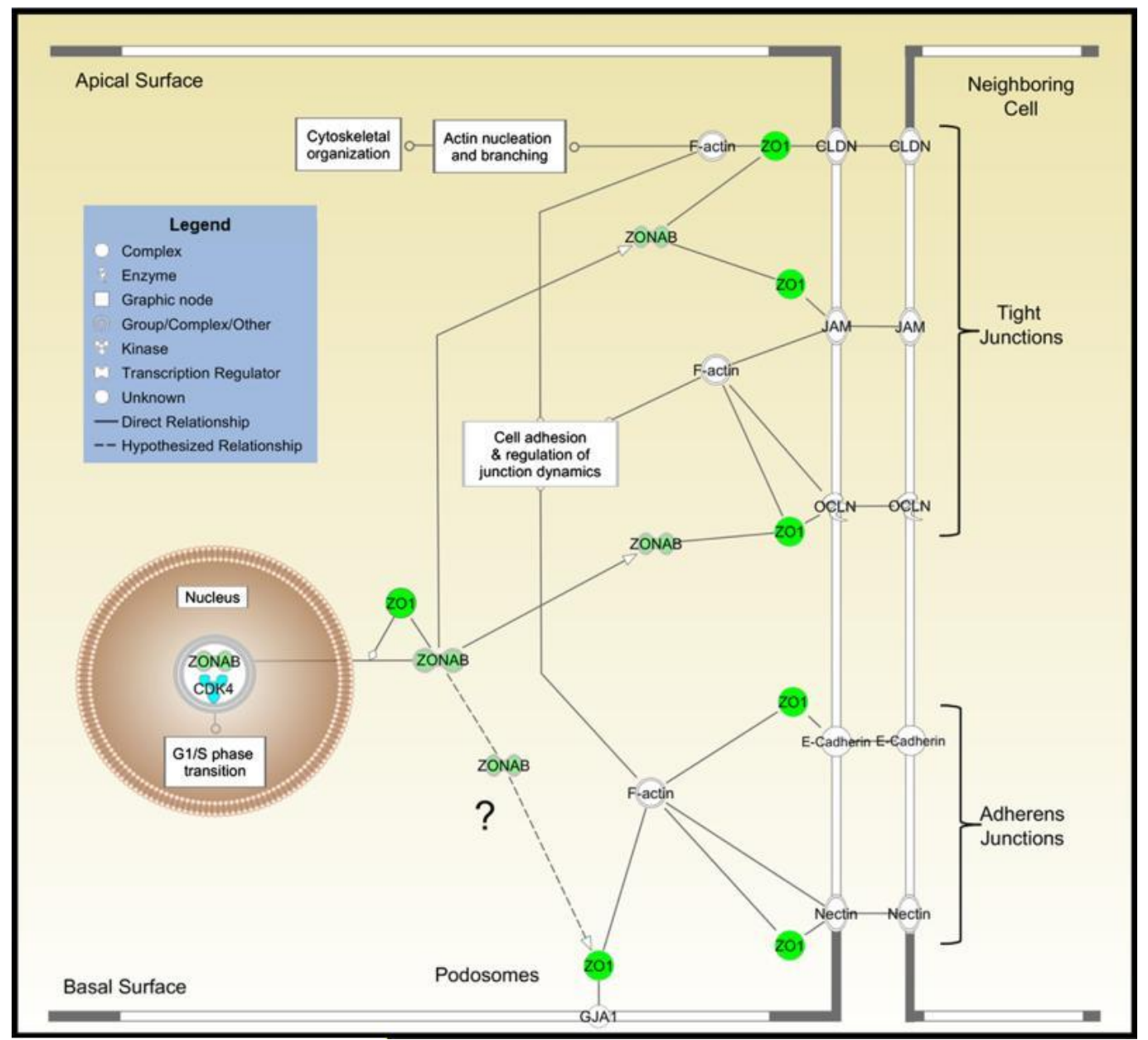

Figure 5: Proposed cellular signaling pathways and regulatory effects between cellular proliferation and adhesion upon exposure to sub-therapeutic concentrations of digitoxin. 
Strengthening adhesion following exposure to sub-therapeutic concentrations of digitoxin could potentially alter actin nucleation/branching and thus cytoskeleton dynamics [58]. Previous studies have shown that changes in adhesion and cell-cell contacts in neoplastic cells play a key role in cancer cell progression and metastasis regulation [59,60]. Such changes can further result in enhancement or re-establishment of the normal cellular function of the epithelial cells as well as re-establishment of cell-to-cell junctions [61, 62].

Our results thus provide new evidence of digitoxin's potential role in regulating proadhesion through enhancement of CDK4/ZONAB/ZO-1 signaling as one of the basic mechanisms for controlling its anti-neoplastic effects. Our results are likely to open new avenues in which the suppression of such a signaling pathway might be broadly applied for the next generation of systematic screening of natural compounds with anti-cancer capabilities.

\section{Conclusions}

Our experimental approach intended to correlate the structural and morphological characteristics of cells exposed to digitoxin (as recorded by the ECIS system) with their functional and metabolic changes (as demonstrated using standard microscopy and cell biology assays). This correlation was made possible by the advantages of the ECIS system that allowed direct, continuously and noninvasively monitoring of NCI-H460 behavior before and after exposure to digitoxin. Our combinatorial analysis showed that exposure to toxic, therapeutic and subtherapeutic concentrations of digitoxin targets cancer cells in a dose and time-dependent manner. Specifically, toxic and therapeutic concentrations activate anti-proliferative cellular mechanisms, whereas sub-therapeutic concentrations of digitoxin increase cellular adhesion. Sequestering CDK4 in the cell cytoplasm reduces cancer cell progression. Understanding the underlying anti- 
neoplastic effects associated with exposure to digitoxin can expedite the potential implementation of this $\mathrm{CG}$ as a chemotherapeutic agent.

\section{Acknowledgements}

The authors acknowledge NanoSAFE and National Science Foundation/EPS-1003907 for their support and Applied Biophysics for the technical support. 


\section{Supporting Information}

\section{Chapter 2: Real Time Analysis of the Effects of Toxic, Therapeutic and Sub- therapeutic Concentrations of Digitoxin on Lung Cancer Cells}

\section{Materials and Methods}

\section{Electric cell-substrate impedance sensing (ECIS)}

Real-time quantification of cellular behavior was conducted using an electric cell impedance sensing instrument (ECIS-Z $\Theta$, Applied Biophysics, NY). In one set of experiments, two ECIS arrays (8W10E+), each containing 8-wells with 40 gold electrodes, were simultaneously employed to provide concomitant measurements of 16 samples at multiple frequencies. Prior to any experiment, the gold electrodes were stabilized for $3 \mathrm{~h}$ in $400 \mu \mathrm{l}$ RPMI media to account for electrode variances and to create a reference line associated with free electrodes; subsequently, the array holder was placed in a humidified incubator at $37{ }^{\circ} \mathrm{C}$ and $5 \% \mathrm{CO}_{2}$ to provide optimal conditions for cellular growth. NCI-H460 cells were added at a density of $2 \times 10^{5}$ cell/mL in a volume of $400 \mu \mathrm{l} /$ well. Cells were allowed to settle and grow over the gold electrodes and form a confluent monolayer for $24 \mathrm{~h}$. The formation of the cellular monolayer was indicated as a settlement in the resistance value with minor fluctuation caused by cellular micromotion. Upon monolayer formation cells were treated with $0,10,25,40$ or $80 \mathrm{nM}$ digitoxin and their cellular behavior was monitored for $48 \mathrm{~h}$ post-exposure.

\section{Western Blot analysis of ZONAB}

Cells were seeded overnight in 6 -well plates at a density of $6 \times 10^{5}$ cell/well, and treated 
with 0,10 or $25 \mathrm{nM}$ digitoxin in $0.1 \%$ DMSO for $24 \mathrm{~h}$. Subsequently, cells were placed on ice and lysed for 30 min in a lysis buffer containing 2\% Triton X-100, 1\% sodium dodecyle sulfate (SDS), 100 mM Sodium Chloride ( $\mathrm{NaCl}), 10 \mathrm{mM}$ Tris-Hydrochloric acid (HCl), Complete Mini cocktail protease inhibitors (all reagents are purchased from Roche, IN) and 1 mM EDTA. Insoluble cellular debris was pelleted by centrifugation at $4^{\circ} \mathrm{C}$ and $16,000 \mathrm{~g}$ for $15 \mathrm{~min}$. The supernatant was collected and used to determine the total protein content using standard Bicinchoninnic acid assay (Thermo Scientific, IL). Briefly, working reagent was prepared according to the manufacturer instructions by mixing 50 parts of reagent A with 1 part of reagent B (reagents included with kit). Two $\mu \mathrm{L}$ of each sample was added to a $96-$ well plate and incubated with $200 \mu \mathrm{L}$ of the working reagent at $37^{\circ} \mathrm{C}$ for $30 \mathrm{~min}$; experiments were performed in duplicate. Control calibration curves were prepared using serial dilutions of standard bovine serum albumin (BSA). Absorbance at 562nm was recorded on a BioTek 96-plate reader (BioTek, Winooski, VT). The supernatant was separated by a 10\% SDS-PAGE gel and transferred to polyvinylidene fluoride (PVDF) membranes using the iBlot ${ }^{\circledR}$ Dry Blotting System (Invitrogen, CA). Membranes were blocked in 5\% skim milk in Tris-buffered saline (TBST, $25 \mathrm{mM}$ Tris- $\mathrm{HCl}, 125 \mathrm{mM} \mathrm{NaCl}$, and 0.1\% Tween-20; Sigma Chemicals, MO) for $1 \mathrm{~h}$ at room temperature, and subsequently incubated with anti-ZONAB primary antibody (Cell Signaling, MA) at $4^{\circ} \mathrm{C}$ overnight. The membranes were subsequently washed three times in phosphate buffer saline (PBS; Lonza, MD) containing 1\% Tween-20 for 10 min each, incubated with horseradish peroxidase-conjugated secondary antibody (Cell Signaling, MA) for $1 \mathrm{~h}$ at room temperature, then washed again for three more times each for $10 \mathrm{~min}$ in TBST. Finally, the samples were analyzed by chemiluminescence (Supersignal West Pico; IL). Band quantification via densitometry was performed using ImageJ software, version 10.2. Cellular exposure to digitoxin did not lead to significant changes in the expression of the ZONAB 


\section{Results and Discussion}

Figure S1 shows the representative behavior of the cells as recorded during the ECIS measurement; changes in the behavior of the cells (i.e., resistance) were recorded every $180 \mathrm{sec}$ for the duration of the experiments. Region A relates to the cellular settling and attachment $(\mathrm{t}<2$ h) and is comprised of more than 35 time points, each time point being an average of 16 replicates. Region B (log growth) and region C (hindered growth) are comprised of more than 60 points and 75 points respectively, each point is the average of 16 replicates. Finally, region D (plateau) is comprised of more than 300 points each with 16 replicates.

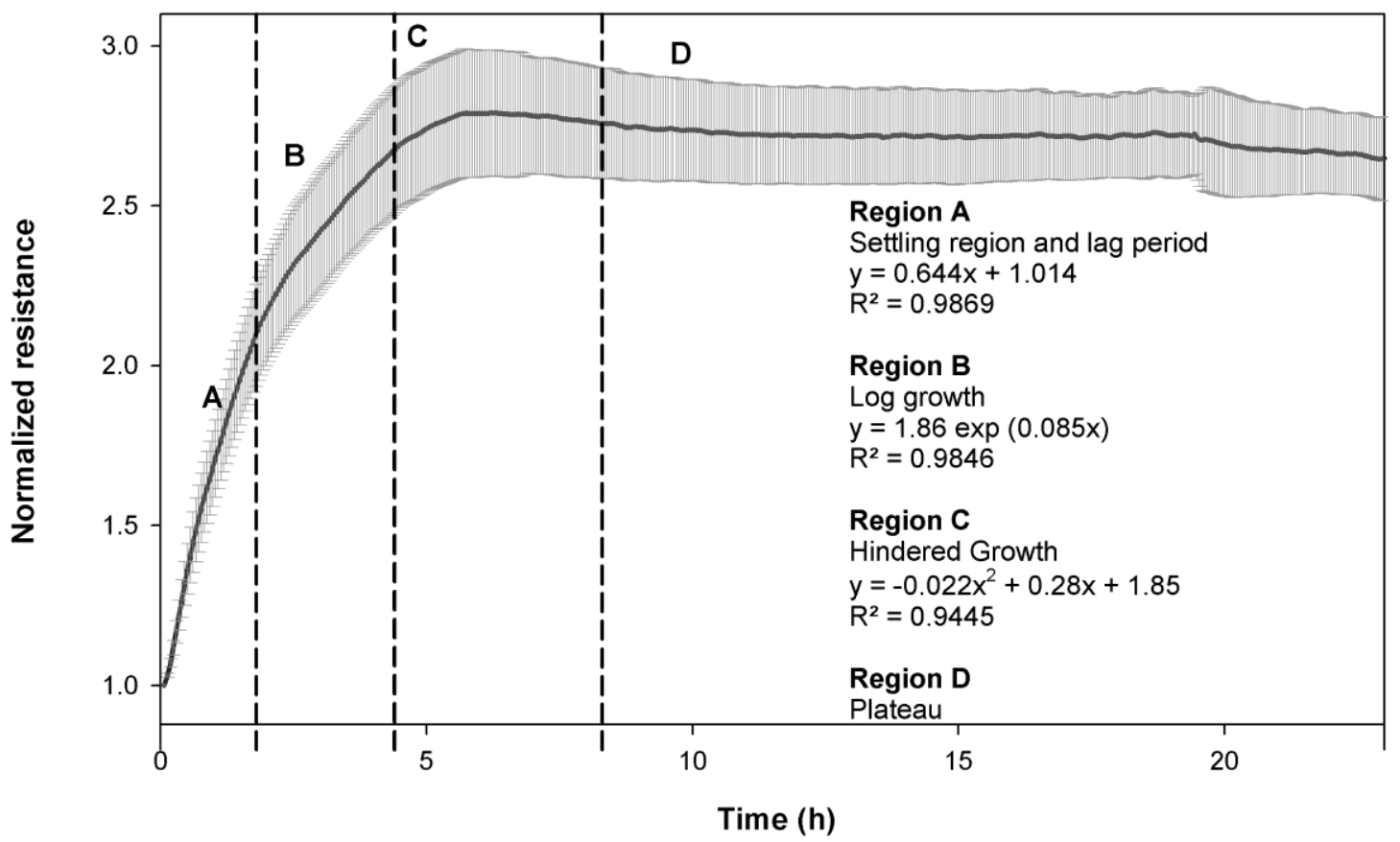


Figure S1: Representative real-time measurements of the normalized resistance associated with the attachment and spreading behavior of the cells measured in real time (error bars are collected every $180 \mathrm{sec}$ for the duration of the experiment).

Figure $\mathrm{S} 2$ shows quantification of the expression of ZONAB after $24 \mathrm{~h}$ exposure to 10 and $25 \mathrm{nM}$ of digitoxin. Results showed that digitoxin exposure did not affect the expression of ZONAB.

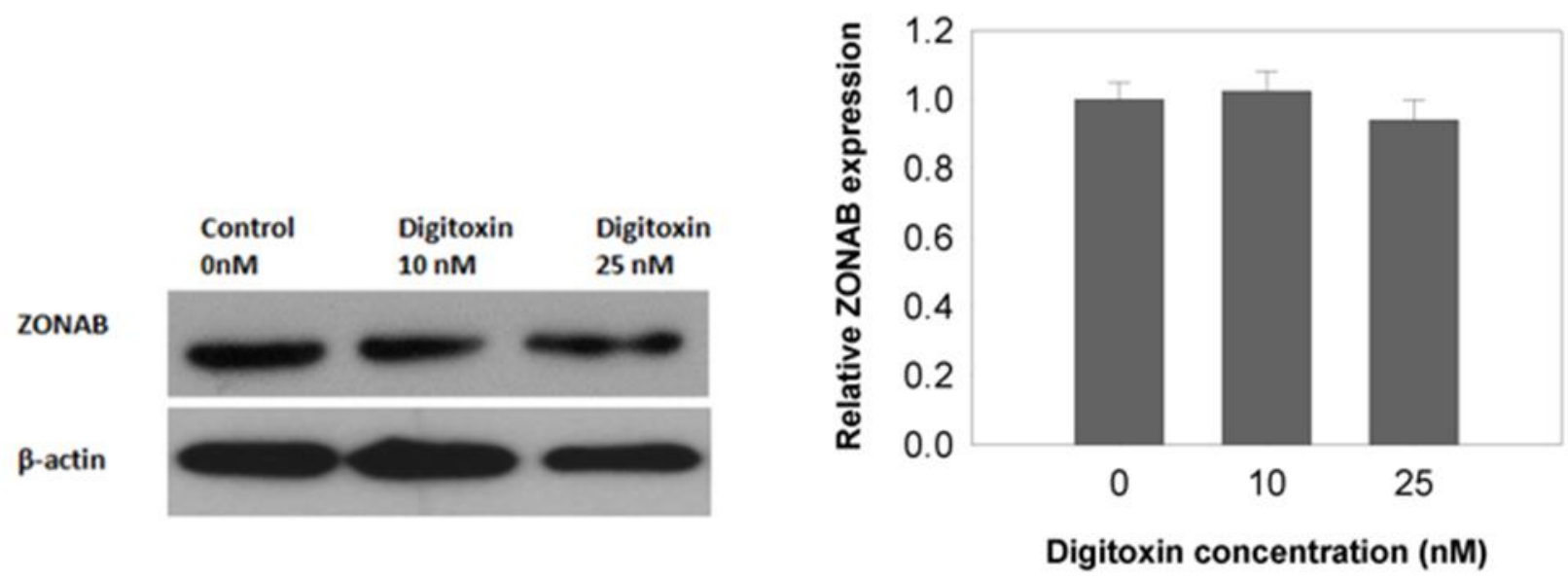


Figure S2: A) Western blot analysis showing ZONAB expression relative to $\beta$-actin 24 h post exposure to 10 and $25 \mathrm{nM}$ Digitoxin. B) Quantification of ZONAB expression levels relative to $\beta$ actin. 


\section{References}

1. Elbaz, H. A., T. A. Stueckle, W. Tse, Y. Rojanasakul, and C. Z. Dinu, Digitoxin and its analogs as novel cancer therapeutics. Exp Hematol Oncol, 2012. 1(1): p. 4.

2. Storstein, L., A. K. Nore, and O. Sjaastad, Studies on digitalis. 23. Blood-brain barrier of digitoxin in humans. Clinical Cardiology, 1979. 2(2): p. 146-150.

3. López-Lázaro, Miguel, Nuria Pastor, Sami S. Azrak, Maria Jesús Ayuso, Felipe Cortés, and Caroline A. Austin, Digitoxin, at concentrations commonly found in the plasma of cardiac patients, antagonizes etoposide and idarubicin activity in K562 leukemia cells. Leukemia Research, 2006. 30(7): p. 895-898.

4. López-Lázaro, Miguel, Digitoxin as an anticancer agent with selectivity for cancer cells: possible mechanisms involved. Expert opinion on therapeutic targets, 2007. 11(8): p. 10431053.

5. Winnicka, Katarzyna, Krzysztof Bielawski, and Anna Bielawska, Cardiac glycosides in cancer research and cancer therapy. Acta poloniae pharmaceutica, 2006. 63(2): p. 109-15.

6. Elbaz, Hosam A., Todd A. Stueckle, Hua-Yu Leo Wang, George A. O'Doherty, David T. Lowry, Linda M. Sargent, Liying Wang, Cerasela Zoica Dinu, and Yon Rojanasakul, Digitoxin and a synthetic monosaccharide analog inhibit cell viability in lung cancer cells. Toxicology and Applied Pharmacology, 2012. 258(1): p. 51-60.

7. Newman, Robert A., Peiying Yang, Alison D. Pawlus, and Keith I. Block, Cardiac Glycosides as Novel Cancer Therapeutic Agents. Molecular Interventions, 2008. 8(1): p. $36-49$.

8. Einbond, Linda Saxe, Masahito Shimizu, Hangbao Ma, Hsan-au Wu, Sarah Goldsberry, Serge Sicular, Maya Panjikaran, Giannicola Genovese, and Erica Cruz, Actein inhibits the 
$\mathrm{Na}+-K+-A T P a s e$ and enhances the growth inhibitory effect of digitoxin on human breast cancer cells. Biochemical and Biophysical Research Communications, 2008. 375(4): p. $608-613$.

9. Prassas, Ioannis, George S. Karagiannis, Ihor Batruch, Apostolos Dimitromanolakis, Alessandro Datti, and Eleftherios P. Diamandis, Digitoxin-Induced Cytotoxicity in Cancer Cells Is Mediated through Distinct Kinase and Interferon Signaling Networks. Molecular Cancer Therapeutics, 2011. 10(11): p. 2083-2093.

10. Sun, K., N. Halberg, M. Khan, U. J. Magalang, and P. E. Scherer, Selective inhibition of hypoxia-inducible factor lalpha ameliorates adipose tissue dysfunction. Mol Cell Biol, 2013. 33(5): p. 904-17.

11. Menger, L., E. Vacchelli, O. Kepp, A. Eggermont, E. Tartour, L. Zitvogel, G. Kroemer, and L. Galluzzi, Trial watch: Cardiac glycosides and cancer therapy. Oncoimmunology, 2013. 2(2): p. e23082.

12. Ho, Anthony K., Valentin Ceña, and David C. Klein, Cardiac glycosides stimulate phospholipase $C$ activity in rat pinealocytes. Biochemical and Biophysical Research Communications, 1987. 142(3): p. 819-825.

13. Jagielska, Joanna, Gustavo Salguero, Bernhard Schieffer, and Udo Bavendiek, Digitoxin elicits anti-inflammatory and vasoprotective properties in endothelial cells: Therapeutic implications for the treatment of atherosclerosis? Atherosclerosis, 2009. 206(2): p. 390396.

14. Pongrakhananon, Varisa, Todd A. Stueckle, Hua-Yu Leo Wang, George A. O’Doherty, Cerasela Zoica Dinu, Pithi Chanvorachote, and Yon Rojanasakul, Monosaccharide digitoxin derivative sensitize human non-small cell lung cancer cells to anoikis through 
Mcl-1 proteasomal degradation. Biochemical Pharmacology, 2014(0).

15. Xie, Zijian and Ting Cai, $N a+/ K+$ ATPase-Mediated Signal Transduction: From Protein Interaction to Cellular Function. Molecular Interventions, 2003. 3(3): p. 157-168.

16. Larre, Isabel, Amparo Lazaro, Ruben G. Contreras, Maria S. Balda, Karl Matter, Catalina Flores-Maldonado, Arturo Ponce, David Flores-Benitez, Ruth Rincon-Heredia, Teresita Padilla-Benavides, $A \bigvee \neq d a$ Castillo, Liora Shoshani, and Marcelino Cereijido, Ouabain modulates epithelial cell tight junction. Proceedings of the National Academy of Sciences, 2010. 107(25): p. 11387-11392.

17. Lopez-Lazaro, M., N. Pastor, S. S. Azrak, M. J. Ayuso, C. A. Austin, and F. Cortes, Digitoxin inhibits the growth of cancer cell lines at concentrations commonly found in cardiac patients. J Nat Prod, 2005. 68(11): p. 1642-5.

18. Wu, S. L., W. Li, A. Wells, and A. Dasgupta, Digoxin-like and digitoxin-like immunoreactive substances in elderly people. Impact on therapeutic drug monitoring of digoxin and digitoxin concentrations. Am J Clin Pathol, 2001. 115(4): p. 600-4.

19. Arndt, Silke, Jochen Seebach, Katherina Psathaki, Hans-Joachim Galla, and Joachim Wegener, Bioelectrical impedance assay to monitor changes in cell shape during apoptosis. Biosensors and Bioelectronics, 2004. 19(6): p. 583-594.

20. Spegel, Christer, Arto Heiskanen, Lars Henrik Dæhli Skjolding, and Jenny Emnéus, Chip Based Electroanalytical Systems for Cell Analysis. Electroanalysis, 2008. 20(6): p. 680702.

21. Giaever, I. and C. R. Keese, Monitoring fibroblast behavior in tissue culture with an applied electric field. Proc Natl Acad Sci U S A, 1984. 81(12): p. 3761-3764.

22. Wegener, Joachim, Ansgar Hakvoort, and Hans-Joachim Galla, Barrier function of porcine 
choroid plexus epithelial cells is modulated by cAMP-dependent pathways in vitro. Brain Research, 2000. 853(1): p. 115-124.

23. Sapper, A., J. Wegener, and A. Janshoff, Cell motility probed by noise analysis of thickness shear mode resonators. Anal Chem, 2006. 78(14): p. 5184-91.

24. Wang, H. S., C. R. Keese, I. Giaever, and T. J. Smith, Prostaglandin E2 alters human orbital fibroblast shape through a mechanism involving the generation of cyclic adenosine monophosphate. J Clin Endocrinol Metab, 1995. 80(12): p. 3553-60.

25. Itagaki, K., Y. Adibnia, S. Sun, C. Zhao, T. Sursal, Y. Chen, W. Junger, and C. J. Hauser, Bacterial DNA induces pulmonary damage via TLR-9 through cross-talk with neutrophils. Shock, 2011. 36(6): p. 548-52.

26. Melnikova, Vladislava O., Krishnakumar Balasubramanian, Gabriel J. Villares, Andrey S. Dobroff, Maya Zigler, Hua Wang, Frederik Petersson, Janet E. Price, Alan Schroit, Victor G. Prieto, Mien-Chie Hung, and Menashe Bar-Eli, Crosstalk between Protease-activated Receptor 1 and Platelet-activating Factor Receptor Regulates Melanoma Cell Adhesion Molecule (MCAM/MUC18) Expression and Melanoma Metastasis. Journal of Biological Chemistry, 2009. 284(42): p. 28845-28855.

27. Wang, Hua-Yu Leo, Wenjun Xin, Maoquan Zhou, Todd A. Stueckle, Yon Rojanasakul, and George A. O'Doherty, Stereochemical Survey of Digitoxin Monosaccharides. ACS Medicinal Chemistry Letters, 2010. 2(1): p. 73-78.

28. Mijatovic, T., V. Mathieu, J. F. Gaussin, N. De Neve, F. Ribaucour, E. Van Quaquebeke, P. Dumont, F. Darro, and R. Kiss, Cardenolide-induced lysosomal membrane permeabilization demonstrates therapeutic benefits in experimental human non-small cell lung cancers. Neoplasia, 2006. 8(5): p. 402-412. 
29. Mijatovic, Tatjana, Anne Op De Beeck, Eric Van Quaquebeke, Janique Dewelle, Francis Darro, Yvan de Launoit, and Robert Kiss, The cardenolide UNBS1450 is able to deactivate nuclear factor $\kappa B$-mediated cytoprotective effects in human non-small cell lung cancer cells Molecular Cancer Therapeutics, 2006. 5(2): p. 391-399.

30. Gulappa, Thippeswamy, Ramadevi Subramani Reddy, Suman Suman, Alice M. Nyakeriga, and Chendil Damodaran, Molecular interplay between cdk4 and p21 dictates G0/G1 cell cycle arrest in prostate cancer cells. Cancer Letters, 2013. 337(2): p. 177-183.

31. Si, Xiaohui and Zheng Liu, Expression and significance of cell cycle-related proteins Cyclin Dl, CDK4, p27, E2F-l and Ets-1 in chondrosarcoma of the jaws. Oral Oncology, 2001. 37(5): p. 431-436.

32. Baggot, J. D. and L. E. Davis, Plasma protein binding of digitoxin and digoxin in several mammalian species. Research in veterinary science, 1973. 15(1): p. 81-7.

33. Giaever, I. and C. R. Keese, Micromotion of mammalian cells measured electrically. Proc Natl Acad Sci U S A, 1991. 88(17): p. 7896-900.

34. Yuan, Bao-Zhu, Amy M. Jefferson, Lyndell Millecchia, Nicholas C. Popescu, and Steven H. Reynolds, Morphological changes and nuclear translocation of DLC1 tumor suppressor protein precede apoptosis in human non-small cell lung carcinoma cells. Experimental Cell Research, 2007. 313(18): p. 3868-3880.

35. Furukawa, Kazuhiro, Tomoko Aida, Yuki Nonaka, Shinichi Osoda, Candido Juarez, Tsuneyoshi Horigome, and Shin Sugiyama, BAF as a caspase-dependent mediator of nuclear apoptosis in Drosophila. Journal of Structural Biology, 2007. 160(2): p. 125-134.

36. Wegener, Joachim, Charles R. Keese, and Ivar Giaever, Electric Cell-Substrate Impedance Sensing (ECIS) as a Noninvasive Means to Monitor the Kinetics of Cell Spreading to 
Artificial Surfaces. Experimental Cell Research, 2000. 259(1): p. 158-166.

37. Chen, Szi-Wen, Jen Ming Yang, Jhe-Hao Yang, Shu Jyuan Yang, and Jong-Shyan Wang, $A$ computational modeling and analysis in cell biological dynamics using electric cellsubstrate impedance sensing (ECIS). Biosensors and Bioelectronics, 2012. 33(1): p. 196203.

38. Xiao, Caide and John H. T. Luong, Assessment of cytotoxicity by emerging impedance spectroscopy. Toxicology and Applied Pharmacology, 2005. 206(2): p. 102-112.

39. Giaever, I. and C. R. Keese, A morphological biosensor for mammalian cells. Nature, 1993. 366(6455): p. 591-2.

40. Stolwijk, Judith A., Christoph Hartmann, Poonam Balani, Silke Albermann, Charles R. Keese, Ivar Giaever, and Joachim Wegener, Impedance analysis of adherent cells after in situ electroporation: Non-invasive monitoring during intracellular manipulations. Biosensors and Bioelectronics, 2011. 26(12): p. 4720-4727.

41. Perrone, Angela, Anna Capasso, Michela Festa, Ether Kemertelidze, Cosimo Pizza, Alexandre Skhirtladze, and Sonia Piacente, Antiproliferative steroidal glycosides from Digitalis ciliata. Fitoterapia, 2012. 83(3): p. 554-562.

42. Wang, Yan, Qiang Qiu, Jia-Jia Shen, Dian-Dong Li, Xue-Jun Jiang, Shu-Yi Si, RongGuang Shao, and Zhen Wang, Cardiac glycosides induce autophagy in human non-small cell lung cancer cells through regulation of dual signaling pathways. The International Journal of Biochemistry \& Cell Biology, 2012. 44(11): p. 1813-1824.

43. Berthet, Cyril, Kimberly D. Klarmann, Mary Beth Hilton, Hyung Chan Suh, Jonathan R. Keller, Hiroaki Kiyokawa, and Philipp Kaldis, Combined Loss of Cdk2 and Cdk4 Results in Embryonic Lethality and Rb Hypophosphorylation. Developmental Cell, 2006. 10(5): p. 
563-573.

44. Jia, Xiaowei, Bingci Liu, Xianglin Shi, Ai Gao, Baorong You, Meng Ye, Fuhai Shen, and Hongju $\mathrm{Du}$, Inhibition of benzo(a)pyrene-induced cell cycle progression by all-trans retinoic acid partly through cyclin D1/E2F-1 pathway in human embryo lung fibroblasts. Cell Biology International, 2006. 30(2): p. 183-189.

45. Puyol, Marta, Alberto Martín, Pierre Dubus, Francisca Mulero, Pilar Pizcueta, Gulfaraz Khan, Carmen Guerra, David Santamaría, and Mariano Barbacid, A Synthetic Lethal Interaction between K-Ras Oncogenes and Cdk4 Unveils a Therapeutic Strategy for Nonsmall Cell Lung Carcinoma. Cancer Cell, 2010. 18(1): p. 63-73.

46. Balda, Maria S. and Karl Matter, Epithelial cell adhesion and the regulation of gene expression. Trends in Cell Biology, 2003. 13(6): p. 310-318.

47. Bauer, H., J. Zweimueller-Mayer, P. Steinbacher, A. Lametschwandtner, and H. C. Bauer, The dual role of zonula occludens (ZO) proteins. J Biomed Biotechnol, 2010. 2010: $\mathrm{p}$. 402593.

48. Kremerskothen, J., M. Stolting, C. Wiesner, A. Korb-Pap, V. van Vliet, S. Linder, T. B. Huber, P. Rottiers, E. Reuzeau, E. Genot, and H. Pavenstadt, Zona occludens proteins modulate podosome formation and function. FASEB J, 2011. 25(2): p. 505-14.

49. Tsukita, S., Tight Junctions, in Encyclopedia of Biological Chemistry, W.J. Lennarz and M.D. Lane, Editors. 2013, Academic Press: Waltham. p. 392-395.

50. Vinken, Mathieu, Elke Decrock, Elke De Vuyst, Raf Ponsaerts, Catheleyne D'Hondt, Geert Bultynck, Liesbeth Ceelen, Tamara Vanhaecke, Luc Leybaert, and Vera Rogiers, Connexins: sensors and regulators of cell cycling. Biochimica et Biophysica Acta (BBA) - Reviews on Cancer, 2011. 1815(1): p. 13-25. 
51. Itoh, M., A. Nagafuchi, S. Moroi, and S. Tsukita, Involvement of ZO-1 in cadherin-based cell adhesion through its direct binding to alpha catenin and actin filaments. J Cell Biol, 1997. 138(1): p. 181-92.

52. Li, Yuanhe, Alan S. Fanning, James M. Anderson, and Arnon Lavie, Structure of the Conserved Cytoplasmic C-terminal Domain of Occludin: Identification of the ZO-1 Binding Surface. Journal of Molecular Biology, 2005. 352(1): p. 151-164.

53. Mandell, Kenneth J., Glenn P. Holley, Charles A. Parkos, and Henry F. Edelhauser, Antibody Blockade of Junctional Adhesion Molecule-A in Rabbit Corneal Endothelial Tight Junctions Produces Corneal Swelling. Investigative Ophthalmology \& Visual Science, 2006. 47(6): p. 2408-2416.

54. Cheung, Isla D., Michel Bagnat, Taylur P. Ma, Anirban Datta, Kimberley Evason, John C. Moore, Nathan D. Lawson, Keith E. Mostov, Cecilia B. Moens, and Didier Y. R. Stainier, Regulation of intrahepatic biliary duct morphogenesis by Claudin 15-like $b$. Developmental Biology, 2012. 361(1): p. 68-78.

55. Priya, Rashmi, Alpha S. Yap, and Guillermo A. Gomez, E-cadherin supports steady-state Rho signaling at the epithelial zonula adherens. Differentiation, 2013. 86(3): p. 133-140.

56. Muller, S. L., M. Portwich, A. Schmidt, D. I. Utepbergenov, O. Huber, I. E. Blasig, and G. Krause, The tight junction protein occludin and the adherens junction protein alphacatenin share a common interaction mechanism with ZO-1. J Biol Chem, 2005. 280(5): p. $3747-56$.

57. Abrams, Charles K. and Steven S. Scherer, Gap junctions in inherited human disorders of the central nervous system. Biochimica et Biophysica Acta (BBA) - Biomembranes, 2012. 1818(8): p. 2030-2047. 
58. González-Mariscal, Lorenza, Susana Lechuga, and Erika Garay, Role of tight junctions in cell proliferation and cancer. Progress in Histochemistry and Cytochemistry, 2007. 42(1): p. 1-57.

59. van Nimwegen, Maroesja J. and Bob van de Water, Focal adhesion kinase: A potential target in cancer therapy. Biochemical Pharmacology, 2007. 73(5): p. 597-609.

60. Ni, Jie, Paul Cozzi, Jingli Hao, Julia Beretov, Lei Chang, Wei Duan, Sarah Shigdar, Warick Delprado, Peter Graham, Joseph Bucci, John Kearsley, and Yong Li, Epithelial cell adhesion molecule (EpCAM) is associated with prostate cancer metastasis and chemo/radioresistance via the PI3K/Akt/mTOR signaling pathway. The International Journal of Biochemistry \& Cell Biology, 2013. 45(12): p. 2736-2748.

61. Coradini, D., C. Casarsa, and S. Oriana, Epithelial cell polarity and tumorigenesis: new perspectives for cancer detection and treatment. Acta Pharmacol Sin, 2011. 32(5): p. 55264.

62. Zhong, Xiaoling and Frederick J. Rescorla, Cell surface adhesion molecules and adhesioninitiated signaling: Understanding of anoikis resistance mechanisms and therapeutic opportunities. Cellular Signalling, 2012. 24(2): p. 393-401. 


\title{
Environmental Science Nano
}

Cite this: Environ. Sci.: Nano, 2014,

\section{Towards elucidating the effects of purified MWCNTs on human lung epithelial cells $\uparrow$}

\author{
Chenbo Dong, $\S \|^{a}$ Reem Eldawud, $\S \|^{a}$ Linda M. Sargent, $\|^{b}$ Michael L. Kashon, $\uparrow^{b}$ \\ David Lowry, $\boldsymbol{q}^{b}$ Yon Rojanasakulq ${ }^{c}$ and Cerasela Zoica Dinu $\|^{\star a}$
}

\begin{abstract}
Toxicity of engineered nanomaterials is associated with their inherent properties, both physical and chemical. Recent studies have shown that exposure to multi-walled carbon nanotubes (MWCNTs) promotes tumors and tumor-associated pathologies and lead to carcinogenesis in model in vivo systems. Herein, we examined the potential of purified MWCNTs used at occupationally relevant exposure doses for particles not otherwise regulated to affect human lung epithelial cells. The uptake of the purified MWCNTS was evaluated using fluorescence activated cell sorting (FACS), while the effects on cell fate were assessed using a 2-(4-iodophenyl)-3-(4-nitrophenyl)-5-(2,4-disulfophenyl)-2H-tetrazolium salt colorimetric assay, cell cycle and nanoindentation. Our results showed that exposure to MWCNTs reduced cell metabolic activity and induced cell cycle arrest. Our analysis further emphasized that the MWCNTs-induced cellular fate result from multiple types of interactions that could be analyzed by means of intracellular biomechani-
\end{abstract}

Received 16th June 2014

Accepted 18th August 2014

DOI: $10.1039 / \mathrm{c} 4 \mathrm{en} 00102 \mathrm{~h}$

rsc.li/es-nano

\section{Nano impact}

Our study showed that exposure of human lung epithelial cells (BEAS-2B) to multi-walled carbon nanotubes (MWCNTs) with user-defined chemical and physical properties reduces the metabolic activity of mitochondria and induces cell cycle arrest. The analysis also demonstrated that the cellular changes induced by such exposure could be analyzed by means of atomic force microscopy and nanoindentation, to help provide insights into the mechanisms of toxicity and nanomaterial-induced cellular transformation.

\section{Introduction}

The aspect-ratio of multi-walled carbon nanotubes (MWCNTs) and their ease of functionalization with drugs and biomolecules were recently shown to increase their

\footnotetext{
${ }^{a}$ Department of Chemical Engineering, West Virginia University, Morgantown WV, 26506, USA. E-mail: cerasela-zoica.dinu@mail.wvu.edu

${ }^{b}$ National Institute for Occupational Safety and Health, Morgantown WV, 26505, USA

${ }^{c}$ Department of Basic Pharmaceutical Sciences, West Virginia University, Morgantown WV, 26506, USA

$\dagger$ Research findings and conclusions are those of the authors and do not necessarily represent the views of the National Institute for Occupational Safety and Health. The authors declare no competing financial interest.

\$ Electronic supplementary information (ESI) available. See DOI: 10.1039/ c4en00102h

$\S$ Equally contributing authors.

I C. D. and R. E. have performed the vast majority of the experiments and drafted the manuscript. D. L. performed the cellular exposure to MWCNTs for the elasticity analysis; L. M. S. has supervised these experiments. M. L. K. has performed the cell cycle and cell elasticity statistical analysis. Y. R. provided guidance in the design of the cell activity assays. C. Z. D. has designed the experiments and finalized the manuscript. All authors have approved the manuscript.
}

cellular delivery for applications in diagnostics, ${ }^{1}$ drug delivery $^{2}$ and cancer therapies. ${ }^{3,4}$ Studies also showed that dispersion stability and loading conditions could influence MWCNT-induced therapeutic effects as well as the efficiency of the loaded drug. ${ }^{5}$ However, such studies failed to reduce the MWCNT-induced inflammatory effects ${ }^{6}$ or to resolve the overall mechanisms of toxicity associated with MWCNT cellular uptake. ${ }^{7-10}$

Purification via strong acid oxidation was recently used as a mean to increase nanotube dispersity and modify both their chemical and physical properties. ${ }^{11}$ Strong acid oxidation shortened the MWCNTs by cutting them at their defect sites, removing impurities and inducing O-derivated functionalities. ${ }^{12}$ Such purified MWCNTs had lower immunological toxicity on BALB/c mice when compared to their impure counterparts (i.e., MWCNTs containing Fe on their external or internal walls).$^{13}$ Complementary, shorter MWCNTs were shown to illicit reduced inflammatory and toxicity responses in the pleural cavity of the mice ${ }^{14}$ Further, the exposure to shorter and purified MWCNTs was shown to lead to changes in the mechanical properties of epithelial cells, ${ }^{15}$ which were used previously as models for nanomaterial toxicity assessment. ${ }^{8}$ 
However, it is still subject to debate to what extent such purified forms of MWCNTs influence cell fate and what are the resulting nanotube-induced cell transformations that could lead to toxicity and cancer development. Failure to assess such effects could potentially jeopardize the implementation of the purified forms in MWCNT-based nanotherapeutics. ${ }^{16}$

By combining conventional biocellular and nanoindentation assays, ${ }^{15}$ we proposed to unravel the cellular changes induced by the exposure to purified MWCNTs, all as a function of the nanomaterial physical and chemical properties. Since the respiratory tract is the primary route of exposure by inhalation, ${ }^{17}$ lung-derived BEAS-2B cells were considered a suitable model system to evaluate MWCNT-induced cellular changes $^{8,15}$ with the exposure dose being determined by extrapolation of in vivo studies mimicking human exposure for 20 weeks at Occupational Safety and Health Administration (OSHA) permissible limits for particles not otherwise regulated. ${ }^{8}$

\section{Materials and methods}

\subsection{Multi-walled carbon nanotube (MWCNT) purification}

MWCNTs (Nanolab Inc., $100 \mathrm{mg}$ ) were purified by ultrasonication (Branson 2510, Fisher Scientific) in a strong mixture of sulfuric (Fisher Scientific, 96.4\%) and nitric (Fisher Scientific, 69.5\%) acids (volume ratio $3: 1$ ) for $1 \mathrm{~h}$ at a temperature of about $23{ }^{\circ} \mathrm{C}$. Afterwards, the mixture was diluted in deionized water (DI water) and filtered through a polycarbonate membrane (Fisher Scientific, GTTP $0.2 \mu \mathrm{m}$ ); the process was repeated several times to remove acid residues or impurities. Purified MWCNTs were collected on a fresh GTTP filter, dried, and stored at room temperature.

\subsection{MWCNT characterization}

Fourier Transform Infrared Spectroscopy (FTIR, Digilab FTS 7000) equipped with a diamond Attenuated Total Reflection (ATR) crystal was used to investigate the chemical properties of pristine and purified MWCNTs. Scans ranging from 1000 to $4000 \mathrm{~cm}^{-1}$ were collected.

Morphology and elemental quantitative analyses of pristine and purified MWCNTs $\left(1 \mathrm{mg} \mathrm{mL}^{-1}\right.$ sample on silica wafer) were performed on a Hitachi S-4700 Field Emission Scanning Electron Microscope (Hitachi High-Technologies Corporation) containing an S-4700 detector combining secondary (SE) and backscattered (BSE) electron detection and operating at $20 \mathrm{KV}$. Energy Dispersive X-Ray Spectroscopy (EDX) results are shown as weight percent of a given element relative to the most dominant element present in the sample.

The average length distribution of the pristine and purified MWCNTs was evaluated using tapping mode Atomic Force Microscopy (AFM) performed in air (Asylum Research, AC240TS, 50 to $90 \mathrm{kHz}$ ). At least 3 scans of $10 \mu \mathrm{m} \times 10 \mu \mathrm{m}$ were acquired for each of the samples being analyzed and a minimum of 30 individual MWCNTs were measured to obtain an average length distribution.

\subsection{Functionalization of MWCNTs with a fluorescent protein}

Alexa 488-labeled Bovine Serum Albumin or Bovine Serum Albumin (Alexa-BSA, or BSA, Invitrogen) were covalently attached to purified MWCNTs using 1-ethyl-3-[3-dimethylaminopropyl] carbodiimide hydrochloride (EDC, Acros Organics) and $\mathrm{N}$-hydroxysuccinimide (NHS, Pierce) chemistry. ${ }^{18}$ Briefly, $2 \mathrm{mg}$ of purified MWCNTs were dispersed in $160 \mathrm{mM}$ EDC and $80 \mathrm{mM}$ NHS (total volume of $2 \mathrm{~mL}$ in 2 -( $N$-morpholino) ethanesulfonic acid sodium salt or MES, $50 \mathrm{mM}$, pH 4.7, (Sigma)) for $15 \mathrm{~min}$ at room temperature with shaking at $200 \mathrm{rpm}$. EDC-NHS activated MWCNTs were subsequently filtered through a GTTP filter membrane, washed thoroughly with MES buffer, and immediately re-dispersed in $2 \mathrm{~mL}$ of $1 \mathrm{mg} \mathrm{mL}$ protein solution in Phosphate Saline Buffer (PBS, Fisher), $100 \mathrm{mM}, \mathrm{pH}$ 7.4. The mixture was incubated for $3 \mathrm{~h}$ at room temperature with shaking at $200 \mathrm{rpm}$. Upon incubation, the resulting protein-based conjugates were filtered and washed extensively with PBS to remove any unbound protein. The supernatant and the first two washes were collected.

\subsection{Protein loading}

The amount of protein bound onto the purified MWCNTs (i.e., protein loaded) was determined using a standard bicinchoninic acid assay (BCA, Fisher). ${ }^{18}$ For this, the working reagent was prepared by mixing 50 parts of reagent $A$ $(1000 \mu \mathrm{L})$, with 1 part of reagent $\mathrm{B}(50 \mu \mathrm{L})$ and subsequently mixing $1000 \mu \mathrm{l}$ of that working reagent with $50 \mu \mathrm{L}$ of either the collected supernatant or the two washes. The resulting solution was gently vortexed and incubated in a water bath at $37^{\circ} \mathrm{C}$ for $30 \mathrm{~min}$. Afterwards, the absorbance values of all the samples were recorded on a spectrophotometer (Evolution 300/600, Thermo Fisher) using $562 \mathrm{~nm}$ as the reading wavelength. Control calibration curves were prepared using serial dilutions of a protein in the working buffer. The relative amount of Alexa-BSA or BSA bound to the purified MWCNTs was estimated from the difference between the amount of protein initially added during the covalent incubation step and the amount of the protein removed in the supernatant and two washes as calculated by the BCA assay.

\subsection{Dispersity analysis}

The dispersity of MWCNTs or MWCNTs functionalized with the proteins was tested both in DI water and in Dulbecco's Modified Eagle Media (DMEM, Invitrogen) containing 10\% Fetal Bovine Serum (FBS, Invitrogen). For this, MWCNTs were sonicated in the testing solution (for a final concentration of $5 \mathrm{mg} \mathrm{mL} \mathrm{m}^{-1}$ ) and subsequently centrifuged at $3000 \mathrm{rpm}$ for $5 \mathrm{~min}$. Part of the corresponding supernatant $(0.8 \mathrm{~mL})$ was collected, filtered through a $0.2 \mu \mathrm{m}$ GTTP filter membrane and then dried under vacuum. The amount of MWCNTs isolated on the filter was weighted and the dispersity was determined relative to the starting amount and volume used per individual sample. 


\subsection{Cell culture}

Immortalized human bronchial epithelial cells (BEAS-2B, ATCC) were cultured in DMEM media containing 10\% FBS, $0.1 \%$ L-glutamine and $1 \%$ penicillin/streptomycin (Invitrogen). The cells were maintained in a humidified atmosphere at $37{ }^{\circ} \mathrm{C}$ and with $5 \% \mathrm{CO}_{2}$; for passaging, a $0.25 \%$ trypsin (Invitrogen) solution was used.

\subsection{Fluorescence activated cell sorting (FACS)}

BEAS-2B cells were seeded for $24 \mathrm{~h}$ in T75 flasks (Fisher) at a density of $3.71 \times 10^{5}$ cells. Subsequently, the cells were exposed to $24 \mu \mathrm{g} \mathrm{cm}^{-2}$ Alexa-BSA-MWCNT conjugates dispersed in fresh media by brief sonication. Control samples, i.e., cells exposed to PBS, free Alexa-BSA at the equivalent amount to the Alexa-BSA amount loaded onto the MWCNTs, and cells exposed to unlabeled MWCNTs, were processed in parallel. Upon $24 \mathrm{~h}$ incubation, the cells in each treatment group were washed with PBS, trypsinized (0.25\% trypsin/ EDTA, Fisher), suspended in DMEM containing 10\% FBS and centrifuged at $1200 \mathrm{rpm}$ for $5 \mathrm{~min}$ to remove free proteins, non-internalized, loosely bound nanotubes or conjugates. Upon centrifugation the samples were washed with PBS, fixed with $100 \mu \mathrm{L}$ of $4 \%$ glutaraldehyde solution (Fisher) for $15 \mathrm{~min}$ at room temperature, and then extensively washed with PBS to remove free glutaraldehyde.

The analyses were performed on a FACS Caliber flow cytometer (Becton Dickinson). The forward scatter (FSC) and the side scatter (SSC) were used to gate the samples to exclude the cellular debris. The FITC signal for the BSA-based conjugates was used at $488 \mathrm{~nm}$ excitation and $515 \mathrm{~nm}$ emission wavelengths. At least 30000 events were recorded for each sample and the data were analyzed and plotted using the FlowJo v7.2.5 software.

\subsection{Cell activity}

BEAS-2B cells were seeded overnight at a density of $1.5 \times$ $10^{4}$ cells into a 96-well plate (Fisher) and exposed for 24, 48 and $72 \mathrm{~h}$ to $24 \mu \mathrm{g} \mathrm{cm} \mathrm{cm}^{-2}$ purified MWCNTs dispersed by brief sonication in the culture media. Afterwards, $10 \mu \mathrm{l}$ of tetrazolium salt (i.e., 2-(4-iodophenyl)-3-(4-nitrophenyl)-5-(2,4disulfophenyl)-2H-tetrazolium also known as WST-1, Roche), was added to each well and the plate was incubated for $2 \mathrm{~h}$ at room temperature. Changes in the color of the individual wells were based on the cell ability to cleave the WST-1 salt to formazan in the presence of the enzyme dehydrogenase and were assessed using a BioTek 96 plate reader (BioTek) and $450 \mathrm{~nm}$ absorbance. The changes are reported as percentage relative activity of exposed versus unexposed cells.

\subsection{Cell cycle analysis}

BEAS-2B cells were seeded overnight in 6 well plates (Fisher) at a concentration of $3 \times 10^{5}$ cells/well, and exposed for $24 \mathrm{~h}$ to $24 \mu \mathrm{g} \mathrm{cm}^{-2}$ purified MWCNTs dispersed in cellular media. Following the exposure to MWCNTs, the cells were trypsinized, collected, washed twice with PBS, centrifuged at $1500 \mathrm{rpm}$ for $6 \mathrm{~min}$ and fixed overnight in $2 \mathrm{~mL} \mathrm{70 \%} \mathrm{ethanol}$ (Fisher) at $-20^{\circ} \mathrm{C}$. Subsequently, the cells were washed again, suspended in $0.2 \%$ Tween 20 (Sigma Chemicals) for $15 \mathrm{~min}$, treated with $10 \mu \mathrm{l} 0.05 \%$ RNase for $15 \mathrm{~min}$ and stained with $30 \mu \mathrm{l}$ propidium iodide (Sigma). Changes in DNA content were determined using a BD LSR Fortessa Flow cell analyzer (BD Biosciences), the BD FACS Diva (Verity Software House) and FlowJo V10.0.7 (Tree Star Inc.) software. The forward scatter (FSC) and the side scatter (SSC) were used to gate the majority of the cell population; 20000 events were collected for each sample. The selection of the cells was based on knowing that in the G0/G1 phase (before DNA synthesis) cells have a defined amount of DNA (i.e., a diploid chromosomal DNA content) and double that amount in the $\mathrm{G} 2$ or $\mathrm{M}$ phase $(\mathrm{G} 2 / \mathrm{M}$, i.e., a tetraploid chromosomal DNA content). Complementary, during the $S$ phase (DNA synthesis), cells contain between one to two DNA levels.

\subsection{Cell nanomechanical changes analysis}

BEAS-2B cells were seeded overnight in $50 \mathrm{~mm} \times 9 \mathrm{~mm}$ parallel culture Petri dishes (BD Biosciences) at a density of $1 \times 10^{5}$ cells per dish. The cells were exposed to $24 \mu \mathrm{g} \mathrm{cm}^{-2}$ purified MWCNTs for $24 \mathrm{~h}$. MFP-3D-BIO AFM (Asylum Research, TE2000-U) was used to evaluate the individual cell Young modulus. The individual cells were selected using optical microscopy and scanned in contact mode in liquid using an Olympus TR400-PB cantilever; the spring constant of the cantilever was measured before each experiment by using a thermal tuning method. ${ }^{19}$ The trigger force was in the nanonewtons range (i.e., 2.3-4.0 nN) while the fitting percentage considered for the data analysis was $90 \%$. The analysis was based on the Sneddon's modification of the Hertz model for a four-sided pyramid ${ }^{20,21}$ with the stiffness being calculated knowing the indentation of the tip and the Poisson's ratio of the cell $(v=0.5) .{ }^{21}$

\subsection{Statistical analysis}

For FTIR, the experiments were repeated at least three times each with two replicates for a total of 6 replicates.

The statistical analyses for the biomechanical and cell cycle experiments were performed with the SAS/STAT software (v9.2) for Windows.

Two-way analysis of variance (ANOVA) and unpaired twotailed Student's $t$-test by SigmaPlot 10.0 (Systat Software Inc.) were used to study the effects of the MWCNTs on the cellular activity and the BSA loading. The experiments were repeated at least three times each with three replicates for a total of 9 replicates.

Differences were considered significant for $p^{*}<0.05$.

\section{Results and discussion}

MWCNTs purification in a sulfuric-nitric acid mixture ${ }^{22}$ was performed to eliminate possible catalyst precursors otherwise 
present during the nanotube synthesis. ${ }^{11}$ The physical and chemical properties of pristine and purified MWCNT samples were investigated using Scanning Electron Microscopy (SEM), Atomic Force Microscopy (AFM), Attenuated Total Reflection Fourier Transform Infrared spectroscopy (ATR-FTIR), and Energy Dispersive X-ray spectroscopy (EDX). SEM analysis showed that the purification did not significantly change the morphology of the MWCNTs (Fig. S1), while AFM analysis showed that the average length of the purified MWCNTs $(792 \pm 254 \mathrm{~nm})$ was about $81 \%$ shorter than that of the pristine MWCNTs $(4261 \pm 2354 \mathrm{~nm}$; Table S1 $)$, which is consistent with previous reports. ${ }^{11}$

Research has shown that shortening of the nanotube leads to the formation of chemical groups such as $\mathrm{COOH},{ }^{23}$ $\mathrm{OH},{ }^{24}$ and $\mathrm{CO}^{12}$ at the nanotube defect sites. Our ATR-FTIR analysis confirmed the presence of the O-containing functional groups (Fig. 1a). The peak at $3370 \mathrm{~cm}^{-1}$ was attributed to the $\mathrm{O}-\mathrm{H}$ stretching vibration ${ }^{25}$ while the peak around $1670 \mathrm{~cm}^{-1}$ was associated with the $\mathrm{C}=\mathrm{O}$ stretching vibration. ${ }^{26}$ In addition, the small peak at around $1330 \mathrm{~cm}^{-1}$ was a result of the $\mathrm{O}-\mathrm{H}$ bending vibration, ${ }^{27}$ and the wide range of the peaks between $1000-1250 \mathrm{~cm}^{-1}$ were associated with the $\mathrm{C}-\mathrm{O}$ stretching vibrations. ${ }^{28}$ The peak around $2000 \mathrm{~cm}^{-1}$ was previously associated with MWCNT functionalization with O-containing groups. ${ }^{29,30}$ The increase in O-containing functional groups and the removal of metal catalysts were confirmed by EDX (Table S2 $\$$ ), with the analysis showing a decrease in the $\mathrm{Fe}$ and $\mathrm{Cu}$ contents for the purified MWCNTs relative to their pristine counterparts. ${ }^{11}$

The dispersity of pristine, purified and protein-MWCNT conjugates was also tested; well-dispersed MWCNTs are required to eliminate mass transfer limitations when studying the interactions of such nanomaterials with cellular systems. ${ }^{31,32}$ Analysis showed that purified MWCNTs were highly dispersed, especially in the DMEM media (Table S3 $\$$ ), presumably due to the: (1) formation of carboxylate anions, and/or (2) their hydrodynamic size. For the first, the negative charges or the carboxylate anions resulted upon nanotube treatment could potentially lead to strong electrostatic repulsion between the individual MWCNTs, ${ }^{33,34}$ while the presence of proteins and amino acids in the media can aid the increased dispersity. ${ }^{35}$ For the second, the shorter nanotubes (as shown by AFM) have a smaller hydrodynamic radius and thus end-toend distances when compared to their pristine counterparts. ${ }^{36}$

Human bronchial respiratory epithelial cells (BEAS-2B) were exposed to $24 \mu \mathrm{g} \mathrm{cm}^{-2}$ of purified MWCNTs covalently functionalized with Alexa-BSA for $24 \mathrm{~h}$; the uptake of fluorescently labeled MWCNTs was evaluated as a change in the forward scatter (FSC) and the side scatter (SSC) of the exposed cells by using a Fluorescence Assisted Cell Sorting (FACS). Cells exposed to purified MWCNTs alone, cells exposed to free Alexa-BSA, and unexposed cells were used as controls. Fig. 1b shows a significantly higher signal for the cells treated with Alexa-BSA-MWCNT conjugates relative to all the control experiments. The higher signal was consistent with the internationalization of the labeled MWCNTs, (a)
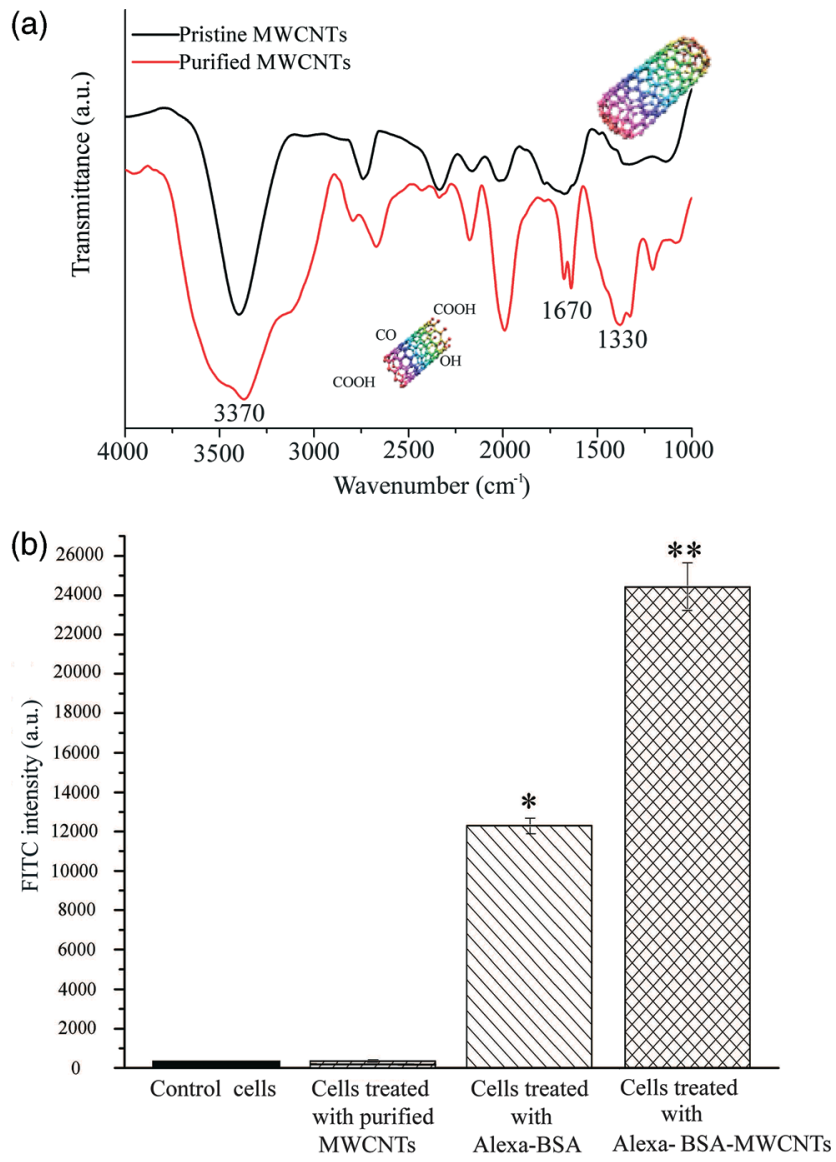

(c)

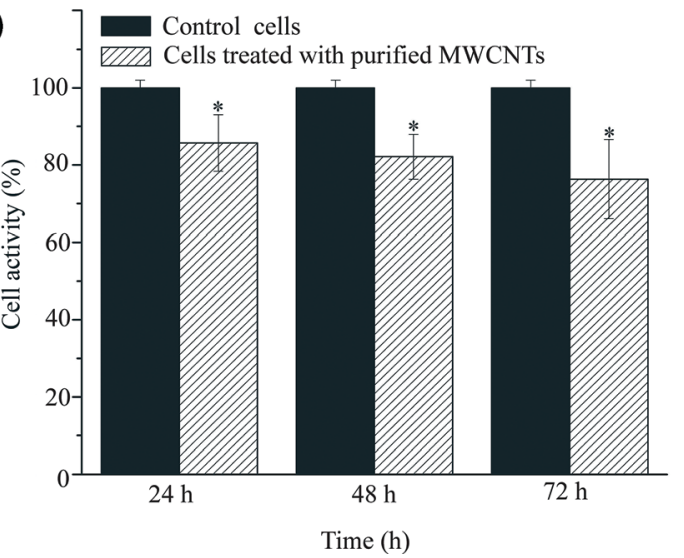

Fig. 1 (a) FTIR spectra of pristine MWCNTs and purified MWCNTs $(n=6)$. (b) FITC intensities of control cells, purified MWCNTs, BEAS-2B cells treated with Alexa-BSA and cells treated with Alexa-BSAMWCNTs conjugates $(n=9)$. (c) Changes in the activity (\%) of the cells exposed to $24 \mu \mathrm{g} \mathrm{cm}^{-2}$ purified MWCNTs. Changes are considered significant for $p^{*}<0.05$.

with a relatively lower intensity observed for the cells exposed to free Alexa-BSA, presumably due to the increased Alexa-BSA susceptibility for proteasomal degradation upon its uptake relatively to the more stable MWCNT-immobilized Alexa-BSA. ${ }^{37-39}$

To investigate the cellular activity upon uptake of the MWCNTs, the exposed cells were assessed using the WST-1 
assay and the mitochondrial dehydrogenase activity was measured (Fig. 1c). Mitochondria are relatively sensitive organelles known to respond to cellular stresses caused by the uptake of CNTs. ${ }^{40}$ WST-1 was preferred to the MTT assay due to existing concerns regarding the CNT interaction with the nonsoluble tetrazolium salt formed in the latter. ${ }^{41}$ Our results showed a significant reduction in the cellular activity of the cells exposed for 24,48 , and $72 \mathrm{~h}$ to the purified MWCNTs. The reduction was presumably due to the internalized MWCNTs initiating mitochondrion stress that could have led to increased concentrations of cytoplasmic $\mathrm{Ca}^{2+42}$ and changes in the mitochondrial permeability transition membrane pore (MPTP) potential. ${ }^{43}$ Studies have shown that when MPTPs are open, the cytochrome $\mathrm{Ca}^{2+}$ pro-apoptotic factor (located in the inner membrane of the mitochondria) and $\mathrm{Ca}^{2+}$ diffuse into the mitochondrial matrix and could trigger caspases-8, -9 and -3 signaling, eventually leading to cell apoptosis. ${ }^{42,44}$

Changes in the cellular activity observed upon the exposure to purified MWCNTs were further translated into changes in the cell cycle progression; ${ }^{45}$ Fig. 2 shows the cell
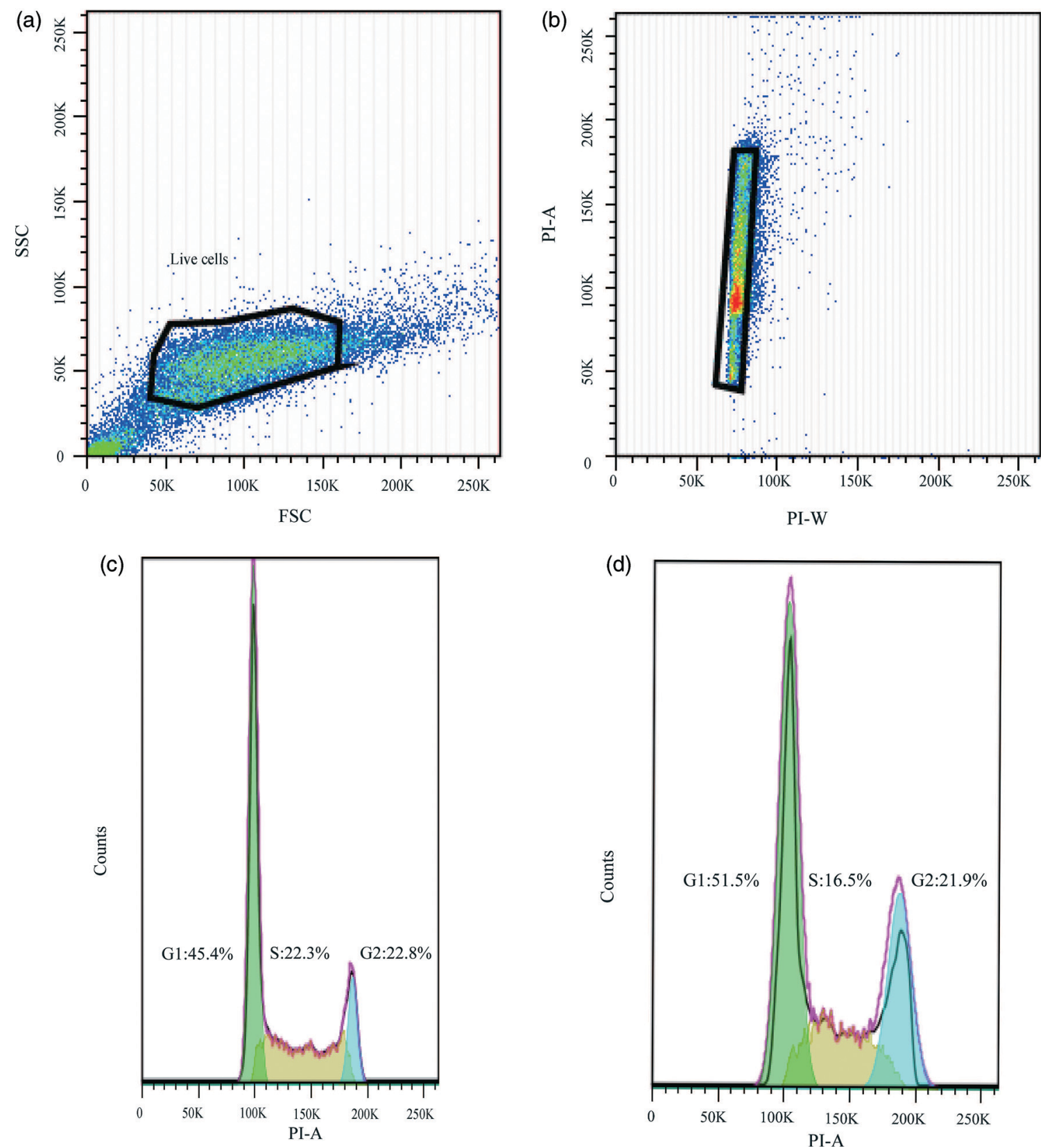

Fig. 2 Fluorescence Activated Cell Sorting (FACS) was used to evaluate the changes in cell cycle upon exposure to purified MWCNTs; $\mathrm{PI}$-stained BEAS-2B cells were used. (a) Forward scatter (FSC) and side scatter (SSC) 2D-plot showing a representative gating of the live cell population. (b) Scatter plot selection of the single cells; representative gating. (c) Cell cycle analysis of the control cells and (d) the cells exposed to purified MWCNTs. 
cycle arrest at the G1/S phase $24 \mathrm{~h}$ after the exposure. Specifically, gating of the cell population (Fig. 2a) or the single cells (Fig. 2b) showed that the cells exposed to purified MWCNTs had a statistically significant increase in G1 (gap) phase $(13 \pm 5.35 \%)$ and a significant decrease in S (synthesis) phase (25 $\pm 6.42 \%$; Fig. $2 \mathrm{~d})$, both relative to controls $\left(p^{*}<0.05\right.$; Fig. 2c) (Table S4 4 ). The increase in the G1 phase could be associated with an extensive change in the DNA content, cell volume, and/or increased synthesis of mRNA and proteins. ${ }^{46}$ The change in the $S$ phase could be associated with a decrease of available cellular $\mathrm{DNA}^{47}$ based on the known affinity of CNTs for nucleic acids. ${ }^{48}$ The observed changes in the cell cycle progression could lead to defects in DNA synthesis and chromosome segregation and hint at the possibility of MWCNTs to induce genotoxicity, ${ }^{8}$ thus complementing previous reports showing CNT-induced chromosomal damage, ${ }^{49}$ generation of reactive oxygen species (ROS), ${ }^{50}$ or multipolar mitotic spindles. ${ }^{8,51}$

Changes in the cellular activity and cell cycle progression were complemented by changes in the cell biomechanics (Fig. 3). Previous studies have shown that changes in cellular biomechanics with an increase in cell deformability correlate with the progression of a cell to a transformed phenotype, i.e., from a benign to a malignant one. ${ }^{52}$ Furthermore, previous research has shown that AFM can be used to investigate biomechanical properties of fixed cells treated with MWCNTs $^{15}$ or to identify cancer cells from a mixture with normal cells, ${ }^{53}$ with reports showing that for an applied force above $7 \mathrm{nN}$ the elastic modulus of a single cell is relatively independent of the tip indentation. ${ }^{54}$

The elastic modulus distributions of the control and MWCNT-exposed live cells (both cell bodies and cell nuclei) are shown in Table 1, with a typical example of a force-indentation $(F-Z)$ curve recorded at the nucleus region shown in Fig. 3a. Compared to the control cells, the cells exposed to purified MWCNTs showed less deformation, suggesting a change in their elastic properties, especially at their nuclear regions where the engaging of the AFM tip was weaker than at the cell edges typically consisting of concentrated cytoskeleton fibers. ${ }^{55}$ The comparison between the elastic modulus of the purified MWCNT-exposed live cells and the control live cells showed a relatively narrow Young's modulus distribution for their nucleus regions (0-6 $\mathrm{kPa})$ and a much wider distribution for the whole cell bodies (0-12 kPa); a higher elastic modulus ( 20 kPa) was observed at the cell periphery and was attributed to the effects induced by the plastic substrate. ${ }^{56}$ Specifically, the average Young's moduli for the whole cell bodies were $2.72 \pm 0.96$ and $3.84 \pm 1.12 \mathrm{kPa}$ for control and exposed live cells, respectively, while the average Young's moduli at their nuclei regions were $1.58 \pm 0.67$ and $2.20 \pm 0.59 \mathrm{kPa}$, respectively (Fig. 3b).

Based on the observed live cell changes, we propose that the cellular uptake of the purified MWCNTs induces a series of concurrent processes (i.e., changes in cellular activity and biomechanical properties), which are functions of the (a)
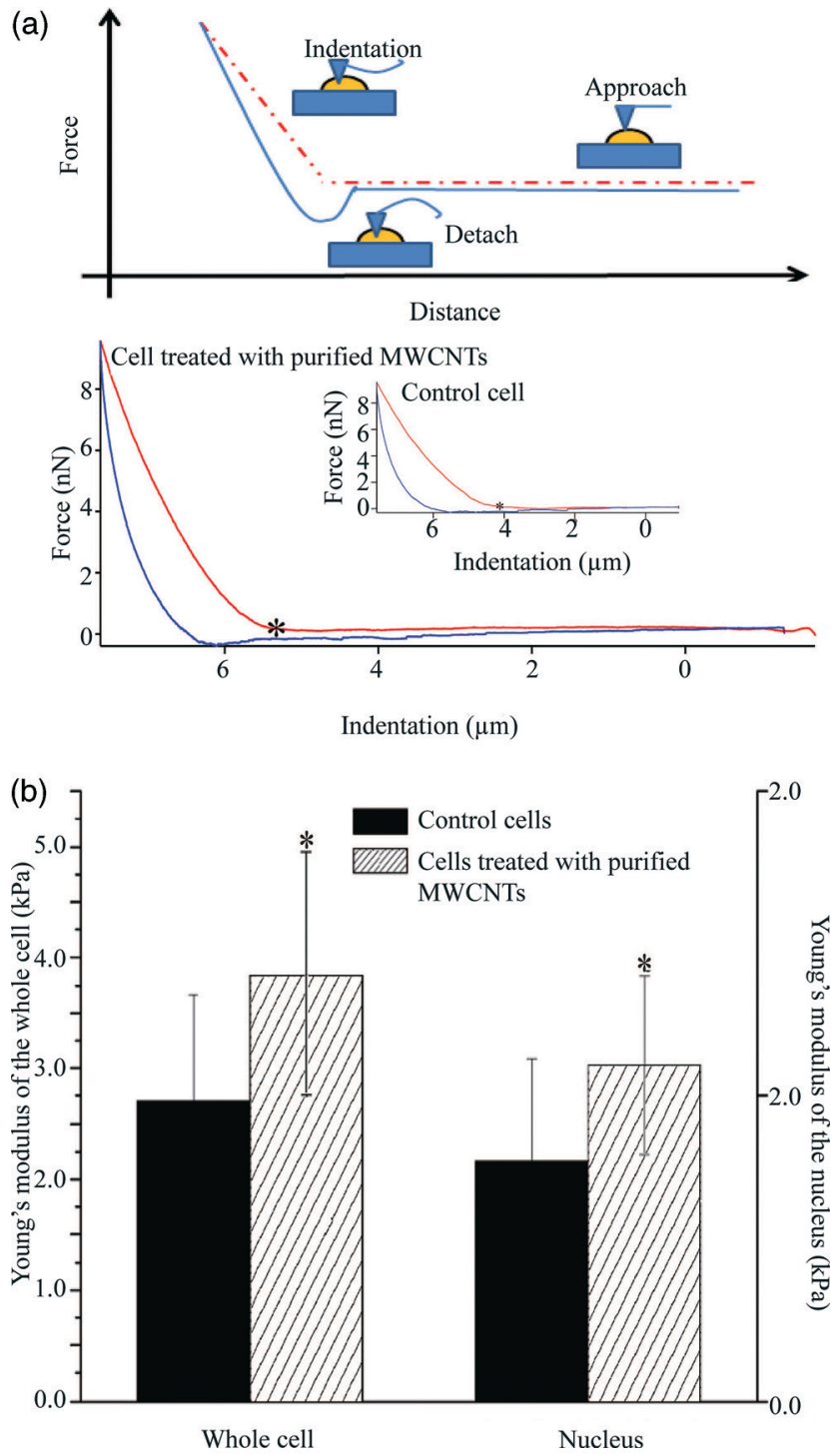

Fig. 3 (a) Schematic diagram of the force-indentation profile of control cells and cells exposed to purified MWCNTs. The red curve follows the approach of the tip to the plastic surface while the blue curve follows the detachment of the tip from the surface. "**" indicates the point at which the tip deflected from the surface to allow the acquisition of the force-indentation measurement profile. (b) Average Young's modulus of the whole live cell and the nucleus region of the control cells and live cells exposed to purified MWCNTs for $24 \mathrm{~h}$. All differences were considered statistically significant for $p^{*}<0.05$.

nanomaterial physical and chemical properties. Such changes could possibly induce cytoskeletal filament reorganization which could lead to increased cellular rigidity, and possible inhibition and/or blockage of the intracellular biomolecular transport or cell cycle progression (Fig. 4). Preliminary research has shown that exposure to MWCNTs led to their cellular integration either into the endosomal structures ${ }^{57}$ or into the cytoskeletal filaments, ${ }^{51}$ and the formation of hybrid-MWCNT filaments. ${ }^{8,15}$ Interestingly, the elastic moduli for the control BEAS-2B cells were higher than previously 
Table 1 Young's modulus distribution of control live cells and live cells exposed to purified MWCNTs

\begin{tabular}{|c|c|c|c|c|c|c|}
\hline Control (whole cells) & 380 & $50.79 \%$ & $31.84 \%$ & $10.79 \%$ & $6.05 \%$ & $0.53 \%$ \\
\hline Cells incubated with purified MWCNTs (whole cells) & 475 & $37.89 \%$ & $35.58 \%$ & $14.32 \%$ & $7.79 \%$ & $0.62 \%$ \\
\hline Cells incubated with purified MWCNTs (nucleus region) & 122 & $57.38 \%$ & $30.07 \%$ & $12.55 \%$ & $0 \%$ & $0 \%$ \\
\hline
\end{tabular}

\section{MWCNTs}

Physical and chemical characterization

\section{Exposure}

Human

bronchial epithelial cells

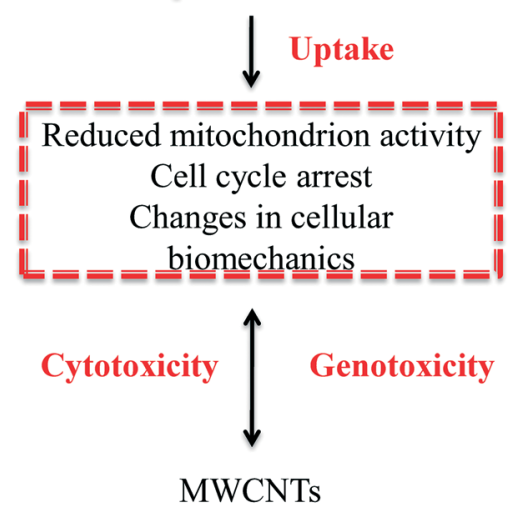

Physical and chemical characterization

Fig. 4 Exposure to purified MWCNTs induced cytotoxic and genotoxic effects by reducing the cellular activity, changing the cell biomechanical properties and disrupting the cell cycle.

reported $(\sim 75 \%)^{55}$ presumably due to variation in the cell culture $^{58}$ or nanoindentation and working conditions. ${ }^{54}$ Our data support recent evidence that relates the cytotoxic and genotoxic effects of MWCNTs to both their physical and chemical properties ${ }^{59}$ and suggests that the occupational exposure to such nanomaterials needs to be fully assessed before implementation in biomedical-related applications is sought.

\section{Conclusions}

Our results showed that purified MWCNT exposure affects the mitochondrial activity, cell biomechanical properties and cell cycle progression in human lung epithelial cells. The analysis further hints at a possible cytotoxic and genotoxic synergism associated with the cellular exposure to MWCNTs, which could potentially induce cell transformation and thus cancer progression.

\section{Acknowledgements}

Support for this research was provided through NanoSAFE, National Science Foundation grant EPS-1003907, NORA 927000Y and the National Institute of Health (NIH; R01-ES022968). Authors acknowledge the use of the WVU Shared Research Facilities and the help of Jeremy Hardinger with the EDX analysis. Flow cytometry experiments were performed in the Flow Cytometry Core Facility at WVU; the Core Facility is in part supported by the National Institute of Health equipment grant number S10OD016165 and the Institutional Development Award (IDeA) from the National Institute of General Medical Sciences of the National Institutes of Health under grant numbers P30GM103488 (CoBRE) and P20GM103434 (INBRE). The authors acknowledge the support and expertise of Dr. Karen Martin, the director of the Core Facility.

\section{References}

1 L. G. Delogu, G. Vidili, E. Venturelli, C. Menard-Moyon, M. A. Zoroddu, G. Pilo, P. Nicolussi, C. Ligios, D. Bedognetti, F. Sgarrella, R. Manetti and A. Bianco, Proc. Natl. Acad. Sci. U. S. A., 2012, 109, 16612-16617.

2 M. Das, R. P. Singh, S. R. Datir and S. Jain, Mol. Pharmaceutics, 2013, 10, 3404-3416.

3 H. C. Tsai, J. Y. Lin, F. Maryani, C. C. Huang and T. Imae, Int. J. Nanomed., 2013, 8, 4427-4440.

$4 \mathrm{~J}$. Li, A. Pant, C. F. Chin, W. H. Ang, C. Menard-Moyon, T. R. Nayak, D. Gibson, S. Ramaprabhu, T. Panczyk, A. Bianco and G. Pastorin, Nanomedicine, 2014, DOI: 10.1016/ j.nano.2014.01.004.

5 L. Jiang, T. B. Liu, H. He, L. A. Pham-Huy, L. L. Li, C. Pham-Huy and D. L. Xiao, J. Nanosci. Nanotechnol., 2012, 12, 7271-7279.

6 T. M. Sager, M. W. Wolfarth, M. Andrew, A. Hubbs, S. Friend, T. H. Chen, D. W. Porter, N. Q. Wu, F. Yang, R. F. Hamilton and A. Holian, Nanotoxicology, 2014, 8, 317-327.

7 E. M. Aneta Fraczek-Szczypta, T. B. Syeda, A. Misra, M. Alavijeh, J. Adu and S. Blazewicz, J. Nanopart. Res., 2012, 14, 1181.

8 K. J. Siegrist, S. H. Reynolds, M. L. Kashon, D. T. Lowry, C. B. Dong, A. F. Hubbs, S. H. Young, J. L. Salisbury, D. W. Porter, S. A. Benkovic, M. McCawley, M. J. Keane, J. T. Mastovich, K. L. Bunker, L. G. Cena, M. C. Sparrow, J. L. Sturgeon, C. Z. Dinu and L. M. Sargent, Part. Fibre Toxicol., 2014, 11, 6, DOI: 10.1186/1743-8977-11-6. 
9 M. Pacurari, X. J. Yin, M. Ding, S. S. Leonard, D. Schwegler-Berry, B. S. Ducatman, M. Chirila, M. Endo, V. Castranova and V. Vallyathan, Nanotoxicology, 2008, 2, 155-170.

10 A. Erdely, M. Dahm, B. T. Chen, P. C. Zeidler-Erdely, J. E. Fernback, M. E. Birch, D. E. Evans, M. L. Kashon, J. A. Deddens, T. Hulderman, S. A. Bilgesu, L. Battelli, D. Schwegler-Berry, H. D. Leonard, W. McKinney, D. G. Frazer, J. M. Antonini, D. W. Porter, V. Castranova and M. K. Schubauer-Berigan, Part. Fibre Toxicol., 2013, 10, 53, DOI: 10.1186/1743-8977-10-53.

11 C. B. Dong, A. S. Campell, R. Eldawud, G. Perhinschi, Y. Rojanasakul and C. Z. Dinu, Appl. Surf. Sci., 2013, 264, 261-268.

12 H. O. Rike Yudianti, Y. Saito, T. Iwata and J. Azuma, Open Mater. Sci. J., 2011, 5, 242-247.

13 S. Koyama, Y. A. Kim, T. Hayashi, K. Takeuchi, C. Fujii, N. Kuroiwa, H. Koyama, T. Tsukahara and M. Endo, Carbon, 2009, 47, 1365-1372.

14 F. A. Murphy, A. Schinwald, C. A. Poland and K. Donaldson, Part. Fibre Toxicol., 2012, 9, 8, DOI: 10.1186/1743-8977-9-8.

15 C. B. Dong, M. L. Kashon, D. Lowry, J. S. Dordick, S. H. Reynolds, Y. Rojanasakul, L. M. Sargent and C. Z. Dinu, Adv. Healthcare Mater., 2013, 2, 945-951.

16 G. M. Rogers-Nieman and C. Z. Dinu, Wiley Interdiscip. Rev.: Nanomed. Nanobiotechnol., 2014, 6, 327-337.

17 R. R. Mercer, J. F. Scabilloni, A. F. Hubbs, L. A. Battelli, W. McKinney, S. Friend, M. G. Wolfarth, M. Andrew, V. Castranova and D. W. Porter, Part. Fibre Toxicol., 2013, 10, 2-11.

18 A. S. Campbell, C. Dong, F. Meng, J. Hardinger, G. Perhinschi, N. Wu and C. Z. Dinu, ACS Appl. Mater. Interfaces, 2014, 6, 5393-5403.

19 B. Ohler, Rev. Sci. Instrum., 2007, 78, 063701.

20 C. Rotsch, K. Jacobson and M. Radmacher, Proc. Natl. Acad. Sci. U. S. A., 1999, 96, 921-926.

21 M. Radmacher, M. Fritz, C. M. Kacher, J. P. Cleveland and P. K. Hansma, Biophys. J., 1996, 70, 556-567.

22 B. Scheibe, E. Borowiak-Palen and R. J. Kalenczuk, Mater. Charact., 2010, 61, 185-191.

23 V. Datsyuk, M. Kalyva, K. Papagelis, J. Parthenios, D. Tasis, A. Siokou, I. Kallitsis and C. Galiotis, Carbon, 2008, 46, 833-840.

24 Z. H. Qu and G. J. Wang, J. Nanosci. Nanotechnol., 2012, 12, 105-111.

25 B. P. Singh, D. Singh, R. B. Mathur and T. L. Dhami, Nanoscale Res. Lett., 2008, 3, 444-453.

26 L. H. Teng and T. D. Tang, J. Zhejiang Univ., Sci., A, 2008, 9, 720-726.

27 C. Moreno-Castilla, M. V. Lopez-Ramon and F. Carrasco-Marin, Carbon, 2000, 38, 1995-2001.

28 L. Wang, L. Ge, T. E. Rufford, J. L. Chen, W. Zhou, Z. H. Zhu and V. Rudolph, Carbon, 2011, 49, 2022-2032.

29 J. Wei, R. Lv, N. Guo, H. G. Wang, X. Bai, A. Mathkar, F. Y. Kang, H. W. Zhu, K. L. Wang, D. H. Wu, R. Vajtai and P. M. Ajayan, Nanotechnology, 2012, 23, 1-5.
30 L. Q. Hoa, M. C. Vestergaard, H. Yoshikawa, M. Saito and E. Tamiya, J. Mater. Chem., 2012, 22, 14705-14714.

31 A. R. Gliga, S. Skoglund, I. O. Wallinder, B. Fadeel and H. L. Karlsson, Part. Fibre Toxicol., 2014, 11, 11, DOI: 10.1186/1743-8977-11-11.

32 F. B. Emilie Brun, G. Veronesi, B. Fayard, S. Sorieul, C. Chanéac, C. Carapito, T. Rabilloud, A. Mabondzo, N. Herlin-Boime and M. Carrière, Part. Fibre Toxicol., 2014, 11, 1-16.

33 Y. T. Shieh, G. L. Liu, H. H. Wu and C. C. Lee, Carbon, 2007, 45, 1880-1890.

34 R. B. Li, X. Wang, Z. X. Ji, B. B. Sun, H. Y. Zhang, C. H. Chang, S. J. Lin, H. Meng, Y. P. Liao, M. Y. Wang, Z. X. Li, A. A. Hwang, T. B. Song, R. Xu, Y. Yang, J. I. Zink, A. E. Nel and T. Xia, ACS Nano, 2013, 7, 2352-2368.

35 C. M. Voge, J. Johns, M. Raghavan, M. D. Morris and J. P. Stegemann, J. Biomed. Mater. Res., Part A, 2013, 101, 231-238.

36 F. A. He and J. T. Fan, Mater. Sci. Technol., 2013, 29, 1423-1429.

37 S. H. Lecker, A. L. Goldberg and W. E. Mitch, J. Am. Soc. Nephrol., 2006, 17, 1807-1819.

38 C. O. Weill, S. Biri, A. Adib and P. Erbacher, Cytotechnology, 2008, 56, 41-48.

39 P. M. Martensen and J. Justesen, BioTechniques, 2001, 30, 782-792.

40 T. Chen, J. J. Zang, H. F. Wang, H. Y. Nie, X. Wang, Z. L. Shen, S. C. Tang, J. L. Yang and G. Jia, J. Nanosci. Nanotechnol., 2012, 12, 8008-8016.

41 J. M. Worle-Knirsch, K. Pulskamp and H. F. Krug, Nano Lett., 2006, 6, 1261-1268.

42 X. Wang, J. Guo, T. Chen, H. Nie, H. Wang, J. Zang, X. Cui and G. Jia, Toxicol. In Vitro, 2012, 26, 799-806.

43 J. J. Lemasters, A. L. Nieminen, T. Qian, L. C. Trost, S. P. Elmore, Y. Nishimura, R. A. Crowe, W. E. Cascio, C. A. Bradham, D. A. Brenner and B. Herman, Biochim. Biophys. Acta, Bioenerg., 1998, 1366, 177-196.

44 W. Zhong, X. Chen, P. Jiang, J. M. F. Wan, P. Qin and A. C. H. Yu, Ultrasound Med. Biol., 2013, 39, 2382-2392.

45 M. J. Berridge, Cell. Signalling Biol., 2012, Module 1, 1-63.

46 C. Bertoli, J. M. Skotheim and R. A. M. de Bruin, Nat. Rev. Mol. Cell Biol., 2013, 14, 518-528.

47 S. Bellucci, S. Dinicola, P. Coluccia, M. Bizzarri, A. Catizone, F. Micciulla, I. Sacco, G. Ricci and A. Cucina, Int. Semicond. Conf., 2012, 2, 37-42.

48 D. Roxbury, X. M. Tu, M. Zheng and A. Jagota, Langmuir, 2011, 27, 8282-8293.

49 L. Migliore, D. Saracino, A. Bonelli, R. Colognato, M. R. D'Errico, A. Magrini, A. Bergamaschi and E. Bergamaschi, Environ. Mol. Mutagen., 2010, 51, 294-303.

50 X. Q. He, S. H. Young, D. Schwegler-Berry, W. P. Chisholm, J. E. Fernback and Q. Ma, Chem. Res. Toxicol., 2011, 24, 2237-2248.

51 L. Rodriguez-Fernandez, R. Valiente, J. Gonzalez, J. C. Villegas and M. L. Fanarraga, ACS Nano, 2012, 6, 6614-6625. 
52 S. Kumar and V. Weaver, Cancer Metastasis Rev., 2009, 28, 113-127.

53 S. E. Cross, Y. S. Jin, J. Rao and J. K. Gimzewski, Nat. Nanotechnol., 2007, 2, 780-783.

54 M. E. Dokukin, N. V. Guz and I. Sokolov, Biophys. J., 2013, 104, 2123-2131.

55 J. Alcaraz, L. Buscemi, M. Grabulosa, X. Trepat, B. Fabry, R. Farre and D. Navajas, Biophys. J., 2003, 84, 2071-2079.
56 J. Rheinlaender, N. A. Geisse, R. Proksch and T. E. Schaffer, Langmuir, 2011, 27, 697-704.

57 Q. X. Mu, D. L. Broughton and B. Yan, Nano Lett., 2009, 9, 4370-4375.

58 M. Nikkhah, J. S. Strobl, E. M. Schmelz and M. Agah, J. Biomech., 2011, 44, 762-766.

59 M. W. Shen, S. H. Wang, X. Y. Shi, X. S. Chen, Q. G. Huang, E. J. Petersen, R. A. Pinto, J. R. Baker and W. J. Weber, J. Phys. Chem. C, 2009, 113, 3150-3156. 


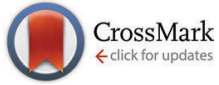

Cite this: J. Mater. Chem. B, 2015, 3, 3983

Received 25th January 2015, Accepted 8th April 2015

DOI: $10.1039 / c 5 t b 00179 j$

www.rsc.org/MaterialsB

\title{
Carbon nanotube uptake changes the biomechanical properties of human lung epithelial cells in a time-dependent manner $\dagger$
}

\author{
Chenbo Dong, $\ddagger^{\mathrm{a}}$ Reem Eldawud, $\ddagger^{\mathrm{a}}$ Linda M. Sargent, ${ }^{\mathrm{b}}$ Michael L. Kashon, \\ David Lowry, ${ }^{\mathrm{b}}$ Yon Rojanasakul ${ }^{\mathrm{c}}$ and Cerasela Zoica Dinu*a
}

\begin{abstract}
The toxicity of engineered nanomaterials in biological systems depends on both the nanomaterial's properties and the exposure duration. Herein we used a multi-tier strategy to investigate the relationship between user-characterized multi-walled carbon nanotubes (MWCNTs) exposure duration and their induced biochemical and biomechanical effects on model human lung epithelial cells (BEAS-2B). Our results showed that exposure to MWCNTs leads to time-dependent intracellular uptake and generation of reactive oxygen species (ROS), along with time-dependent gradual changes in cellular biomechanical properties. In particular, the amount of internalized MWCNTs followed a sigmoidal curve with the majority of the MWCNTs being internalized within $6 \mathrm{~h}$ of exposure; further, the sigmoidal uptake correlated with the changes in the oxidative levels and cellular biomechanical properties respectively. Our study provides new insights into the time-dependent induced toxicity caused by exposure to occupationally relevant doses of MWCNTs and could potentially help establish bases for early risk assessments of other nanomaterials' toxicological profiles.
\end{abstract}

\section{Introduction}

The versatility in physical and chemical properties including high strength to weight ratio, ${ }^{1-3}$ electrical $^{4}$ and thermal conductivity ${ }^{5}$ make multi-walled carbon nanotubes (MWCNTs) attractive candidates for applications in a wide variety of fields from electronic devices, ${ }^{6}$ sports equipment, ${ }^{7}$ aerospace industry, ${ }^{8}$ to sensors ${ }^{9}$ and composite materials. ${ }^{10}$ Recently, functionalization of MWCNTs with amino acids, ${ }^{11}$ peptides, ${ }^{12}$ and other small biomolecules ${ }^{13}$ has been explored for biomedical and biotechnological applications in gene ${ }^{14}$ and drug delivery, ${ }^{15}$ bioimaging, ${ }^{16}$ and for therapeutics. ${ }^{17}$ However, as the list of potential bio-related applications increases, so do concerns regarding MWCNTs potential to induce toxicity in biological systems. ${ }^{18}$

Studies showed that MWCNT interactions with biological systems are dependent on the nanomaterial physico-chemical properties, with MWCNT-induced toxicity being attributed to

\footnotetext{
${ }^{a}$ Department of Chemical Engineering, West Virginia University, Morgantown WV, 26506, USA. E-mail: cerasela-zoica.dinu@mail.wvu.edu

${ }^{b}$ National Institute for Occupational Safety and Health, Morgantown WV, 26505, USA

${ }^{c}$ Department of Basic Pharmaceutical Sciences, West Virginia University,

Morgantown WV, 26506, USA

$\dagger$ Electronic supplementary information (ESI) available. See DOI: 10.1039/ c5tb00179j

\# Equally contributing authors.
}

various material's characteristics including size, ${ }^{19}$ surface charge, ${ }^{20}$ and aggregation state. ${ }^{21}$ Studies also revealed that upon uptake either through piercing ${ }^{22}$ or endocytosis, ${ }^{23}$ MWCNTs translocate the cell altering its physiological properties and fate, by causing cyto and genotoxicity. For instance, research showed that MWCNTs cytoplasmic translocation resulted in reactive oxygen species (ROS) generation ${ }^{24,25}$ and changes in cellular elasticity, ${ }^{26}$ with variations in the cellular mechanical properties indicating cellular transformation and potential for cancer development. ${ }^{26-28}$ Further analysis showed that MWCNTs containing precursor metal catalysts displayed a higher toxicity than their catalyst-free counterparts. ${ }^{29}$ Reduced toxicity was observed upon acid washing and carboxylation of MWCNTs, ${ }^{30}$ with the diminished effects being attributed to the nanotube shorter lengths and higher dispersity resulted from acid cutting at the MWCNT defect sites, as well as from grafting of O-related functionalities. ${ }^{31}$ For genotoxicity, uptake of MWCNTs resulted in their initial accumulation in the cell endosome; ${ }^{32}$ subsequent release in the cytoplasm led to MWCNT interactions with cell nucleus, ${ }^{23}$ polyploidy, ${ }^{33}$ changes in chromosome numbers, ${ }^{34}$ and disruption of mitosis, ${ }^{35}$ just to name a few.

Based on these previous studies showing that MWCNTs uptake and cellular translocation result in complex interactions with cellular components, we now begin to understand how exposure to nanotubes may be involved in the mechanisms responsible of cancer initiation. ${ }^{36}$ However, predicting the time of uptake in relation to the nanomaterial physico-chemical 
properties and its aggregation state, as well as predicting timedependent nanotube-induced cellular fate upon nanomaterial internalization have been challenging. Further, the correlations between early onsets of exposure and immediate cellular responses are still lacking. Assessment of early exposure onsets are required for preventing MWCNT-induced deleterious effects, to reduce the risks for cell transformation or cancer development ${ }^{28,34,36}$ to thus help contribute to the next generation of biomedical applications or products based on these nanomaterials. ${ }^{37}$

The present study aimed to investigate the cellular effects induced by short duration (1-12 h) exposure to occupational relevant doses of MWCNTs as derived from recommendations made by the Occupational Safety and Health Administration (OSHA) for particles less than $5 \mu \mathrm{m}$ in diameter. ${ }^{28}$ By using a combination of analytical techniques relying on fluorescence activated cell sorting (FACS), Atomic Force Microscopy (AFM) nanoindentation and a versatile human lung epithelial cells (BEAS-2B) model, ${ }^{26,28}$ we assessed MWCNT-induced cellular changes and investigated how early cellular uptake and MWCNT cytoplasmic accumulation lead to biomechanical and biochemical cell transformations. We aimed to help explain cellular fate as well as to advance the possibility to set norms for early onsets risk assessment of this nanomaterial.

\section{Materials and methods}

\section{Multi-walled carbon nanotubes (MWCNTs) washing}

Commercial multi-walled carbon nanotubes (MWCNTs; 95\% purity, PD15L5-20, 10-20 $\mathrm{nm}$ in diameter, 1-5 $\mu \mathrm{m}$ in length) purchased from Nanolab Inc. were washed in a mixture of $3: 1(\mathrm{v} / \mathrm{v})$ concentrated sulfuric (96.4\%, Fisher, USA) and nitric (69.5\%, Fisher, USA) acids using established protocols. ${ }^{31}$ Briefly, the MWCNT-acids mixture was sonicated in an ice bath sonicator (Branson 2510, Fisher, USA) at a temperature lower than $23{ }^{\circ} \mathrm{C}$ for $1 \mathrm{~h}$. Subsequently, the mixture was diluted in deionized (di) water $(2 \mathrm{~L})$, and filtered through a GTTP $0.2 \mu \mathrm{m}$ polycarbonate filter membrane (Millipore, Fisher, USA). The acid washed MWCNTs (simply called MWCNTs) isolated on the filter membrane were subsequently redispersed in di water; filtration cycles were repeated at least 3 other times to remove acid traces and any released impurities or dissociated catalysts. The nanotubes isolated on the filter membrane were subsequently dried in a vacuum desiccator and stored at room temperature until further use.

\section{Characterization of MWCNTs}

A combination of analytical techniques was employed to determine MWCNT physical and chemical properties. Briefly, energy dispersive X-ray spectroscopy (EDX) was used to investigate the elemental composition of the MWCNTs; the analysis was performed on a Hitachi S-4700 field emission scanning electron microscope (USA) combining secondary (SE) and backscattered (BSE) electron detection, and operating in a single unit at $20 \mathrm{kV}$.

Dispersity of MWCNTs was evaluated by suspending MWCNT samples in three different dispersing agents, i.e. di water, phosphate buffer (PBS, $\mathrm{pH}=7.4$, Invitrogen, USA) and Dulbecco's
Modified Eagle Media (DMEM, Invitrogen, USA) with or without $10 \%$ fetal bovine serum (FBS, Invitrogen, USA). The corresponding solutions ( $3 \mathrm{mg} \mathrm{mL}^{-1}$ of sample) were centrifuged at $3000 \mathrm{rpm}$ for $5 \mathrm{~min}$; subsequently, $0.8 \mathrm{~mL}$ of each of the supernatant was removed and filtered through a $0.2 \mu \mathrm{m}$ GTTP membrane. The sample isolated on the filter membrane was dried under vacuum and the amount of MWCNTs retained on the filter was weighed. The sample dispersity was calculated in relation to the starting volume and amount of dry MWCNTs on the filter membrane.

The length distribution of MWCNTs was evaluated using an Atomic Force Microscope (AFM) with Si tips operating in air and in tapping mode (Asylum Research, AC240TS, 50 to $90 \mathrm{kHz}$, USA). The average length of the MWCNT samples was evaluated by investigating the length of 30 individual MWCNTs from 3 areas, each of $10 \mu \mathrm{m} \times 10 \mu \mathrm{m}$.

A Renishaw InVia Raman Spectrometer (CL532-100, $100 \mathrm{~mW}$, USA) was used to investigate MWCNT chemical structure. Briefly, $1 \mathrm{mg}$ of sample was mounted onto clean glass substrates (Fisher, USA) and irradiated using an argon ion $\left(\mathrm{Ar}^{+}\right)$laser beam operating at $514.5 \mathrm{~nm}$ and having a spot size below $0.01 \mathrm{~mm}^{2}$. To reduce sample heating effects the exposure time was kept at $10 \mathrm{~s}$; the scan range was set between 100 and $3200 \mathrm{~cm}^{-1}$.

\section{Generation of MWCNT-based conjugates}

Alexa 488 bovine serum albumin 488 (Alexa-BSA; Sigma, USA) was covalently immobilized onto MWCNTs using 1-ethyl-3-[3dimethylaminopropyl]carbodiimide hydrochloride (EDC, Acros Organics, USA) and $\mathrm{N}$-hydroxysuccinimide (NHS, Pierce, USA) chemistry. ${ }^{31,38,39}$ Briefly, $2 \mathrm{mg}$ of MWCNTs were dispersed in $160 \mathrm{mM}$ EDC and $80 \mathrm{mM}$ NHS (total volume of $2 \mathrm{~mL}$ ) in (2- $N$-morpholino) ethanesulfonic acid sodium salt (MES buffer, $50 \mathrm{mM}, \mathrm{pH} 4.7$, Sigma, USA) for $15 \mathrm{~min}$ at room temperature on a bench shaker operating at $200 \mathrm{rpm}$. EDC/NHS activated MWCNTs were subsequently filtered through a $0.2 \mu \mathrm{m}$ GTTP filter membrane, washed thoroughly with MES buffer to remove ester residues, and immediately re-dispersed in $2 \mathrm{~mL}$ of $1 \mathrm{mg} \mathrm{mL}$ Alexa-BSA solution in PBS, $\mathrm{pH} 7.4$ for $3 \mathrm{~h}$ at room temperature with shaking at $200 \mathrm{rpm}$. When the time elapsed, the resulting Alexa-BSA-MWCNT conjugates were filtered and washed extensively with PBS to remove any unbound protein. The supernatant and the first two washes were collected to help quantify the amount of protein washed out (i.e., amount of protein not bound to the MWCNTs).

\section{Protein loading onto MWCNTs}

The amount of Alexa-BSA immobilized onto MWCNTs (i.e., loaded protein) was determined using standard colorimetric bicinchoninic acid assay (BCA, Pierce, USA) and subtracting the amount of protein washed out in the supernatant and the first two washes from the amount of protein offered to the MWCNTs during the initial incubation step. Briefly, the working reagent was prepared by mixing 50 parts of reagent $\mathrm{A}(1000 \mu \mathrm{L})$, with 1 part of reagent $B(20 \mu \mathrm{L})$. Next, $1000 \mu \mathrm{L}$ of the working reagent was mixed with $50 \mu \mathrm{L}$ of the supernatant or the isolated washes. The resulting solution was vortexed lightly and incubated in a water bath at $37{ }^{\circ} \mathrm{C}$ for $30 \mathrm{~min}$. Subsequently, the sample 
absorbance was monitored at $562 \mathrm{~nm}$ (Spectrophotometer, Evolution 300/600, Thermo Fisher, USA) and compared to the absorbance of known concentrations of free Alexa-BSA in PBS mixed with the working buffer.

\section{Emission efficiency of the loaded protein}

The emission efficiency of the Alexa-BSA loaded onto the MWCNTs surface was evaluated using a spectroscopical assay. ${ }^{38,40}$ Briefly, the absorbance spectrum of $100 \mu \mathrm{g} \mathrm{mL}{ }^{-1}$ of Alexa-BSA-MWCNT conjugates was recorded relative to both the absorbance spectrum of unlabeled MWCNTs $\left(100 \mu \mathrm{g} \mathrm{mL}^{-1}\right)$ and the absorbance spectrum of free Alexa-BSA at an amount equivalent to the amount loaded onto the MWCNTs. The absorbance spectrum of immobilized Alexa-BSA (excitation at $488 \mathrm{~nm}$ and emission at $515 \mathrm{~nm}$ ) was quantified by subtracting the values of the unlabeled MWCNTs from the absorbance values of the Alexa-BSA-MWCNTs, with the efficiency of emission being determined as the height of the absorbance peaks of the bound protein relative to the height of the peaks of free protein in solution.

\section{Cell culture}

Human bronchial epithelial cells (BEAS-2B, ATCC, USA) were cultured in DMEM media supplemented with 10\% FBS, $2 \mathrm{mM}$ L-glutamine and 100 units per $\mathrm{mL}$ penicillin/streptomycin (reagents were purchased from Invitrogen, USA). Cells were passaged regularly using $0.25 \%(\mathrm{w} / \mathrm{v})$ trypsin (Invitrogen, USA) with $1.5 \mathrm{mM}$ EDTA (Molecular Probes, USA) and maintained in a humidified atmosphere at $37{ }^{\circ} \mathrm{C}$ and under $5 \% \mathrm{CO}_{2}$.

\section{Fluorescence activated cell sorting (FACS) analysis}

Cells in DMEM media with 10\% FBS were seeded in T75 flasks (Fisher, USA) for $24 \mathrm{~h}$ at a density of $3.71 \times 10^{5}$ cells and

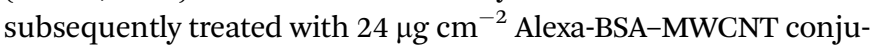
gates or unlabeled MWCNTs, each dispersed using a brief bath sonication (5 $\mathrm{s}$ in $1 \mathrm{~s}$ increments) in fresh media. Cellular exposure was performed for different time periods, i.e., 1, 3, 6 or $12 \mathrm{~h}$ respectively; control samples of untreated cells or cells exposed to free Alexa-BSA at an equivalent amount to the amount loaded onto the MWCNTs were also performed.

For uptake analysis, cells were washed with fresh PBS, collected using $0.25 \%$ trypsin, suspended in DMEM with $10 \%$ FBS and centrifuged at 1200 for $5 \mathrm{~min}$ to remove noninternalized or loosely bound Alexa-BSA-MWCNT conjugates, free MWCNTs or free Alexa-BSA in solution respectively. Subsequently the samples were washed with PBS, pelleted, and incubated at room temperature for $15 \mathrm{~min}$ in $100 \mu \mathrm{L}$ of glutaraldehyde solution (4\%, Sigma, USA). Upon incubation, the samples were washed again with PBS and analyzed using a FACS Caliber flow cytometer (Becton Dickinson, USA). The forward scatter (FSC) and side scatter (SSC) were adjusted using control cells to lay in a range of $0-1000$; gating included the majority of the live cell population. FITC signal for the Alexa-BSA or derivate MWCNT-based conjugates used excitation at $488 \mathrm{~nm}$ and emission at $515 \mathrm{~nm}$. At least 10000 events were contained in the gated area and data was analyzed and plotted using Flow Jo v7.2.5 software.

\section{Reactive oxygen species (ROS) evaluation}

Intracellular ROS generation was determined using the oxidationsensitive fluorescent probe $2^{\prime}, 7^{\prime}$-dichlorofluorescein diacetate (DCF-DA; Sigma, USA). Upon formation of ROS, cellular esterase hydrolyzes DCF-DA to the highly fluorescent $2^{\prime}, 7^{\prime}$-dichlorofluorescein (DCF). ${ }^{41}$ Briefly, BEAS-2B cells were seeded overnight in a 96-well plate (Corning, USA) at a density of $1.5 \times 10^{4}$ cell per well, and incubated with $5 \mu \mathrm{M}$ DCF-DA at $37{ }^{\circ} \mathrm{C}$ for $30 \mathrm{~min}$. Subsequently, the cells were treated with $24 \mu \mathrm{g} \mathrm{cm}{ }^{-2}$ MWCNTs suspended in Hank's balanced salt solution (HBSS; Life Technologies, USA) for different time periods. Control samples of unexposed cells or cells exposed to pristine MWCNTs were also investigated. Quantification of the intracellular levels of ROS was performed using a multi-well plate reader (FLUOstar OPTIMA, BMG LABTECH Inc., USA) and evaluating the DCF fluorescence at $485 \mathrm{~nm}$ excitation and $520 \mathrm{~nm}$ emission respectively.

\section{Biomechanical analysis}

Cells were seeded at a density of $10^{5}$ cells in $50 \mathrm{~mm} \times 9 \mathrm{~mm}$ Petri dishes (BD Biosciences, USA) and exposed to $24 \mu \mathrm{g} \mathrm{cm} \mathrm{cm}^{-2}$ of MWCNTs for 1, 3, 6 or $12 \mathrm{~h}$, respectively. The exposed cells were washed twice with PBS ( 5 min for each washing step) and fixed with $4 \%$ glutaraldehyde solution for $30 \mathrm{~min}$. Three additional washing steps were performed to remove free glutaraldehyde; the Petri dishes were subsequently filled with PBS and used for elasticity analysis. Elastic modulus analysis of the BEAS-2B cells exposed to MWCNTs or of the unexposed control cells were performed using an AFM integrated with an inverted fluorescence microscope (MFP-3D-BIO AFM, Asylum Research, USA). The nanomechanical properties of control cells and cells exposed to MWCNTs were evaluated using Sneddon's modification of the Hertz model developed for a four-sided pyramid. ${ }^{42-44}$ Forcedisplacement curves acquired during elastic mapping were converted into force-indentation curves ${ }^{45}$ based on the assumption that the indentation depth of the sample is extremely thick. ${ }^{46}$ The cells elasticity (Young modulus) was evaluated knowing the indentation of the tip, $\delta$, the Poisson's ratio $\nu$ for the cell $\left(0.5^{44}\right)$ and the opening angle of the tip, i.e., $\alpha=36^{\circ}$. The cantilever was calibrated against a plastic substrate and its exact spring constant was determined using thermal tuning method prior every experiment. ${ }^{47}$ The trigger force was in the nanonewtons range (i.e., 2.8-4.6 nN) and the fitting percentage used for all experiments analysis was $90 \%$.

\section{Statistical analysis}

Alexa-BSA loading onto the MWCNTs and Alexa-BSA functionality analysis were repeated six times. FACS experiments were performed in triplicates and repeated three times. The triplicate samples were averaged to provide three independent replications. ROS experiments were performed for four independent replicates and repeated three times for a total of minimum 12 samples per each time point. All results are presented as mean \pm standard deviation. Two-way analysis of variance (ANOVA) and unpaired two-tailed Student's T-test were performed using JMP 8.0 (SAS Institute, USA) and Sigma Plot 10.0 (Systat Software Inc., USA). 
The elasticity experiments were performed using a complete randomized block design with each block containing two cell culture dishes; three independent blocks were run for each one of the experimental onsets being investigated. For each culture, six individual randomly selected cells were examined for the biomechanical analysis and averaged into a single value as cells from the same culture are not independent. The variables were analyzed using the proc mixed procedure in SAS/Stat for Windows (SAS) with experimental block modeled as a random effect.

All differences were considered statistically significant for $p^{*}<0.05$.

\section{Results and discussion}

A multi-tier strategy was used to investigate the cellular responses to differential uptake of user-characterized multi-walled carbon nanotubes (MWCNTs). The strategy involved time-controlled exposures of model human lung epithelial cells (BEAS-2B) ${ }^{26,28}$ to occupationally relevant doses of $24 \mu \mathrm{g} \mathrm{cm} \mathrm{cm}^{-2}$ of MWCNTs with user-characterized physical and chemical properties. The exposure dose chosen for this investigation was based on previous recommendations made by the Occupational Safety and Health Administration (OSHA) for particles less than $5 \mu \mathrm{m}$ in diameter; ${ }^{28}$ the dose corresponds to human work space exposure of 8 years at $5 \mathrm{mg} \mathrm{m} \mathrm{m}^{-3}$ and was extrapolated from in vivo CNT exposure data normalized to alveolar surface area of mice lungs. ${ }^{28,34,48}$ The proposed multi-tier strategy is aimed to help identify the early exposure onsets-induced cellular fate and potential deleterious effects of MWCNTs thus further our understanding of nanotubeinduced toxicity.

The physical and chemical properties of MWCNTs were determined using energy dispersive X-ray spectroscopy (EDX) for elemental composition and Atomic Force Microscopy (AFM) for nanotubes length. Previous studies have shown that the presence of high contents of metal impurities (e.g., Fe and Ni) in nanotubes could induce mitochondrial membrane damage, generation of reactive oxygen species (ROS), loss in intracellular low molecular weight thiols (GSH) and accumulation of lipid hydroperoxides macrophages. ${ }^{36,37,49}$ Our analysis revealed that the MWCNTs used in this study had minimum traces of Fe and $\mathrm{Cu}$ catalysts (combined, only about $1.50 \mathrm{wt} \%$ ), with the dominant element being carbon (C, $90.25 \mathrm{wt} \%$; Table S1, ESI $\dagger)$. The presence of oxygen $(\mathrm{O}, 7.56 \mathrm{wt} \%)$ in the sample was presumably due the washing of the pristine (as purchased) MWCNTs in the mixture of sulfuric and nitric acids known to graft O-related functional groups. ${ }^{31}$ Such grafting was further responsible for the observed increased dispersity of the nanotubes in different media, all relative to their pristine counterparts (Table S2, ESI $\dagger$ ). Lastly, $\sim 75 \%$ of nanotube shortening was observed by AFM analysis (i.e., $1040 \pm 553 \mathrm{~nm}$ from $4261 \pm 2354 \mathrm{~nm}$ respectively) confirming previous reports (Table S3, ESI $\dagger$ ). ${ }^{26,50}$

Fluorescence activated cell sorting (FACS) was used to evaluate the uptake of the user-characterized MWCNTs. ${ }^{36,51}$ For this MWCNTs were covalently functionalized with 488 Alexa bovine serum albumin (Alexa-BSA) using the O-related functional groups grafted upon acid treatment and a zero-length chemistry; ${ }^{52}$ covalent immobilization of the Alexa-BSA ensured stable protein binding thus eliminating concerns associated with possible protein removal during MWCNTs sonication in cellular media. Alexa-BSA labeling was the preferred method to facilitate FACS analysis of MWCNTs uptake since previous studies showed that the protein does not significantly change the internalization conditions of the nanotubes, with in vitro studies assessing and comparing cellular-induced fate upon exposure to both labeled or unlabeled nanotubes present in media enriched with free amino acids, nutrition factors and BSA. ${ }^{53,54}$

Our loading analysis showed that the amount of Alexa-BSA immobilized onto the MWCNTs was $0.22 \pm 0.06 \mathrm{mg}$ protein per mg MWCNTs, which is comparable to previous published reports. ${ }^{55-57}$ Our dispersity analysis (Table S2, ESI $\dagger$ ) comparing labeled and unlabeled MWCNTs dispersed in cellular media (the cellular media contains free protein and amino acids, with a minimum of $1 \mathrm{mg} \mathrm{mL}^{-1}$ glucose, $7.6 \mathrm{mg} \mathrm{mL}^{-1}$ proteins and $4.6 \mathrm{mg} \mathrm{mL}^{-1}$ albumins respectively ${ }^{58,59}$ ) did not reveal significant changes in the sample dispersity, all relative to control buffer systems, while spectroscopy analysis confirmed that the immobilized Alexa-BSA retained its functionality. In particular, the efficiency of fluorescence emission of the immobilized protein relative to the free protein in solution at the same amount was $34 \pm 9 \%$ (Fig. 1a); the reduced efficiency was presumably due to the protein deformation and quenching at the nanotube interface. ${ }^{38,39}$ Raman spectroscopy also confirmed protein immobilization as a change in the $I_{\mathrm{D}} / I_{\mathrm{G}}$ ratio of Alexa-BSA-MWCNTs (0.967) relative to MWCNTs alone (0.799) (Fig. 1b and Table S4, $\mathrm{ESI} \dagger$ ) where the $I_{\mathrm{D}} / I_{\mathrm{G}}$ represents the degree of disorder in the $\mathrm{C}$-based structure with the $\mathrm{G}$ band being related to the presence of $\mathrm{sp}^{2}$ species ${ }^{60}$ and the $\mathrm{D}$ band being associated with defects and non-crystalline species. ${ }^{61}$

The change in the fluorescence intensity of the cells exposed to Alexa-BSA-MWCNT conjugates relative to controls (i.e., unexposed cells, cells exposed to the same amount of free Alexa-BSA as the amount additionally loaded onto the nanotubes, or cells exposed to MWCNTs) as recorded by FACS is shown in Fig. 2. The analysis showed that within the first hour of exposure (Fig. 2a), the uptake of Alexa-BSA-MWCNTs was relatively slow and did not result in significant changes in the FITC signal of the exposed cells relative to their controls. A higher FITC signal was however observed upon cellular uptake of the free Alexa-BSA; this was presumably associated with the free protein's ability to cross the cellular membrane more efficiently than its MWCNT-immobilized counterpart. ${ }^{62}$ Analysis also showed that longer exposure time led to a gradual increase in the FITC signal of the cells exposed to Alexa-BSA-MWCNT conjugates and free Alexa-BSA respectively, both relative to unexposed cells or cells exposed to MWCNTs (Fig. 2a). Specifically, after $3 \mathrm{~h}$ of exposure there was a 55\% increase in the FITC signal of the cells exposed to Alexa-BSAMWCNTs relative to unexposed cells, a $19 \%$ increase relative to cells exposed to unlabeled MWCNTs, and a $12 \%$ increase relative to cells exposed to free Alexa-BSA. Similarly, after $6 \mathrm{~h}$ of exposure, the FITC signal of the cells exposed to Alexa-BSAMWCNTs showed a $92 \%, 50 \%$, and $20 \%$ change relative to the 
(a)

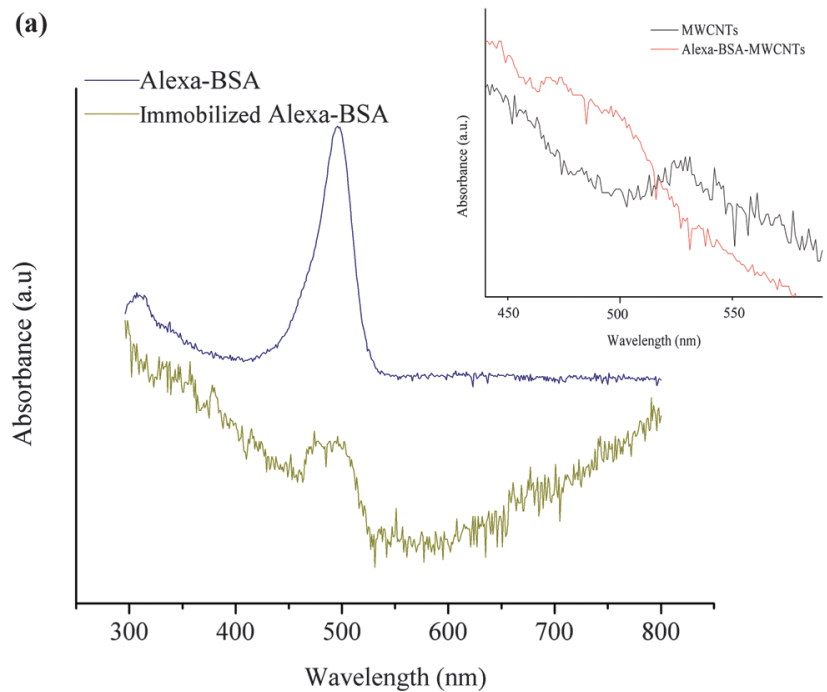

(b)

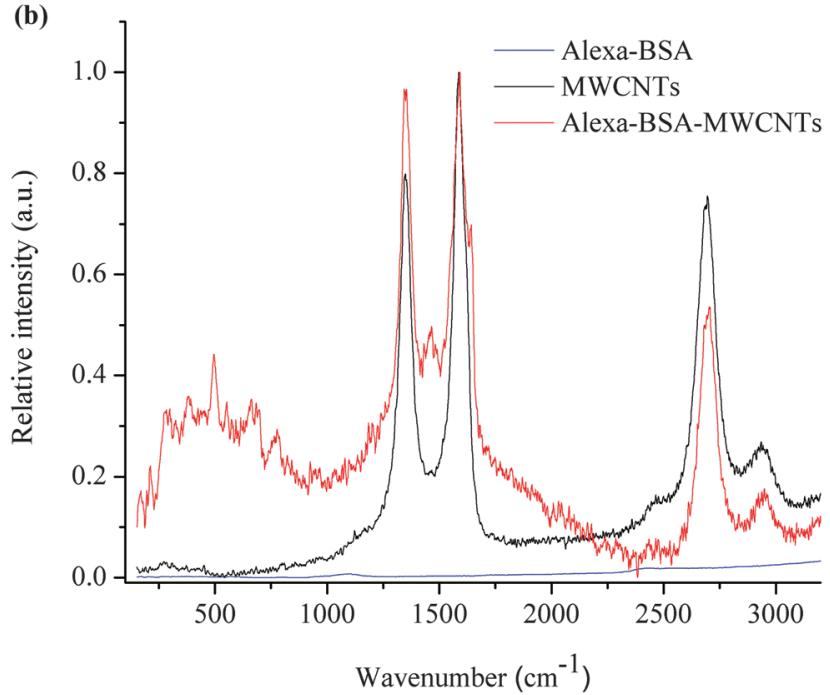

Fig. 1 (a) UV-Vis spectra of free Alexa-BSA and immobilized Alexa-BSA The spectrum of the immobilized Alexa-BSA was obtained by subtracting the absorbance values of the MWCNTs from the ones of the Alexa-BSAMWCNT conjugates both shown in the inset. (b) Raman spectra of free Alexa-BSA, MWCNTs and Alexa-BSA-MWCNT conjugates.

FITC signal of the unexposed cells, cells exposed to unlabeled MWCNTs, or cells exposed to free Alexa-BSA respectively. Finally, after $12 \mathrm{~h}$ exposure the FITC signal of the cells exposed to Alexa-BSA-MWCNTs was about $100 \%$ higher than that of the unexposed cells and cells exposed to unlabeled MWCNTs respectively, and only slightly higher (not statistically significant) than that of the cells exposed to free Alexa-BSA.

The lower FITC signal observed for the cells exposed to free Alexa-BSA relative to the cells exposed to the Alexa-BSA-MWCNT conjugates is presumably due to increased free protein susceptibility for proteosomal degradation ${ }^{63}$ relative to its immobilized counterpart known to have increased stability upon immobilization at the MWCNTs interface. ${ }^{38}$ Although the signal of cells exposed to unlabeled MWCNTs did not yield significant differences relative to unexposed cells at all the time points being investigated, it seemed to have gradually increased with the exposure duration up to $6 \mathrm{~h}$ (a)

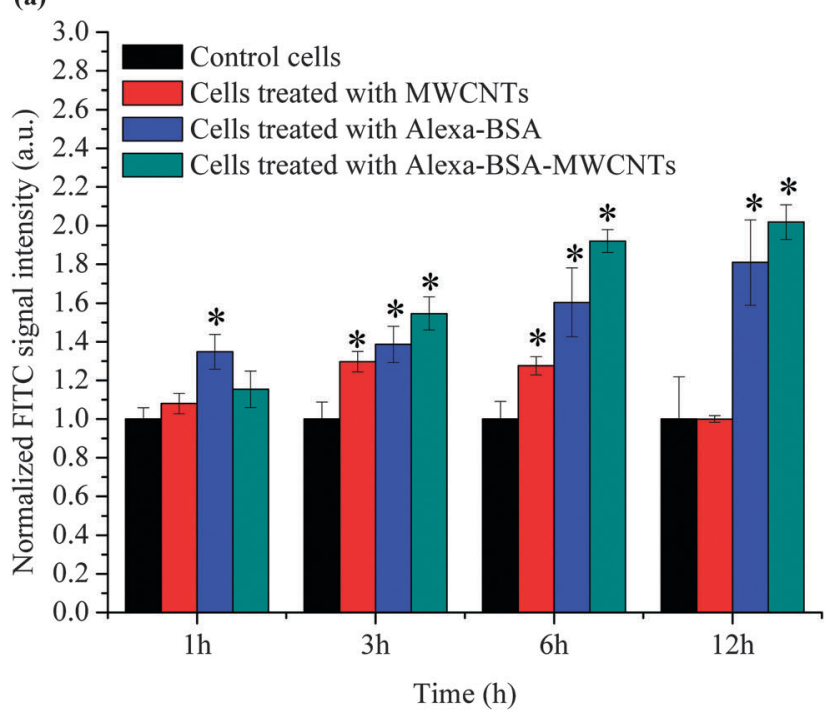

(b)

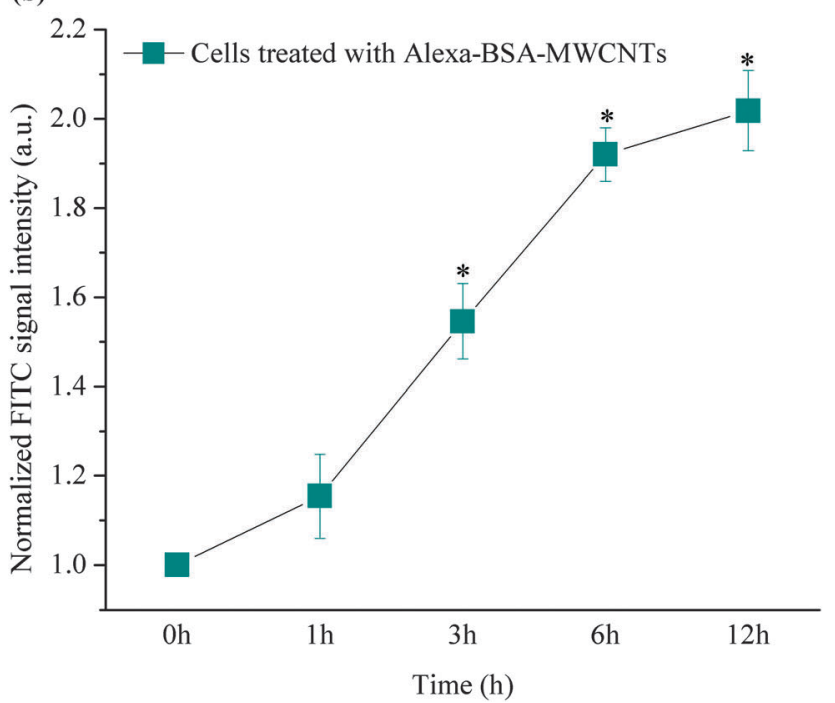

Fig. 2 (a) Normalized FITC signal of control cells, cells exposed to MWCNTs (24 $\mu \mathrm{g} \mathrm{cm}^{-2}$ ), cells exposed to free Alexa-BSA, and cells exposed to AlexBSA-MWCNT conjugates respectively at different time points. FITC signal for the Alexa-BSA or MWCNT-based conjugates was recorded using excitation at $488 \mathrm{~nm}$ and emission at $515 \mathrm{~nm}$; at least 10000 events were contained and analyzed in the gated area. (b) Comparative cross-analysis of normalized FITC signal of cells treated with Alexa-BSA-MWCNT conjugates at different time points. All differences were considered statistically significant for $p^{*}<0.05$.

only to return to its baseline after $12 \mathrm{~h}$. Such trend is presumably associated with increased MWCNTs intracellular translocation, association and co-localization with cellular elements ${ }^{35,64}$ that will interfere with the intrinsic fluorescence of the MWCNTs to thus lead to the observed change in intensity. ${ }^{36,65,66}$

Fig. $2 \mathrm{~b}$ shows the comparative cross-analysis of the normalized fluorescent intensity of the cells exposed to Alexa-BSA-MWCNT conjugates relative to unexposed cells, all as a function of the exposure time. The analysis showed that the uptake of Alexa-BSAMWCNT conjugates followed a three parameter sigmoid curve and increased with the exposure time. Specifically, within the 
first hour of exposure there was a 15\% increase in the fluorescent intensity, while between 1 and $3 \mathrm{~h}$ of exposure the fluorescent intensity of cells exposed to the conjugates increased by $39 \%$ accounting for a total increase of $\sim 54 \%$ in $3 \mathrm{~h}$ time frame, all relative to the intensity of unexposed control cells. Furthermore, between 3 and $6 \mathrm{~h}$ of exposure there was an additional $37 \%$ increase in the fluorescence intensity, while between 6 and $12 \mathrm{~h}$ the increase in intensity was less evident. These results are in agreement with previous reports that showed that the highest increase in CNTs cellular uptake and internalization occurs within $3 \mathrm{~h}$ from initial cellular exposure. ${ }^{32,67}$

We correlated the differential, time-dependent uptake as recorded by FACS with the intracellular oxidative species (ROS) generation using $2^{\prime}, 7^{\prime}$-dichlorofluorescein diacetate (DCF-DA) fluorescence probe. ${ }^{68}$ Our aim was to determine whether the observed time-dependent, differential uptake leads to a gradual formation and accumulation of ROS; the interest in ROS is driven by the current research which shows that superoxide $\left(\mathrm{O}^{2-}\right)$, hydroxyl radical $\left(\mathrm{OH}^{-}\right)$, and nonradical derivatives of $\mathrm{O}$ such as hydrogen peroxide $\left(\mathrm{H}_{2} \mathrm{O}_{2}\right)$ accelerate cell death by damaging cellular components including DNA, proteins, and lipid membranes. ${ }^{69-72}$ ROS generation following MWCNTs exposure was previously shown to lead to changes in the mitochondrial potential and apoptosis signaling activation, ${ }^{71,73}$ with ROS generation depending of acellular factors such as particle surface, size, composition and/or being due to cellular responses resulting from MWCNT-cell interactions.

Our ROS analysis complemented FACS results and showed increased levels in the amount of ROS generated following 1, 3 and $6 \mathrm{~h}$ exposure to MWCNTs and decreased levels similar to those of controls after $12 \mathrm{~h}$ of exposure (Fig. 3). The significant increase in the ROS after $1 \mathrm{~h}$ exposure is associated with the

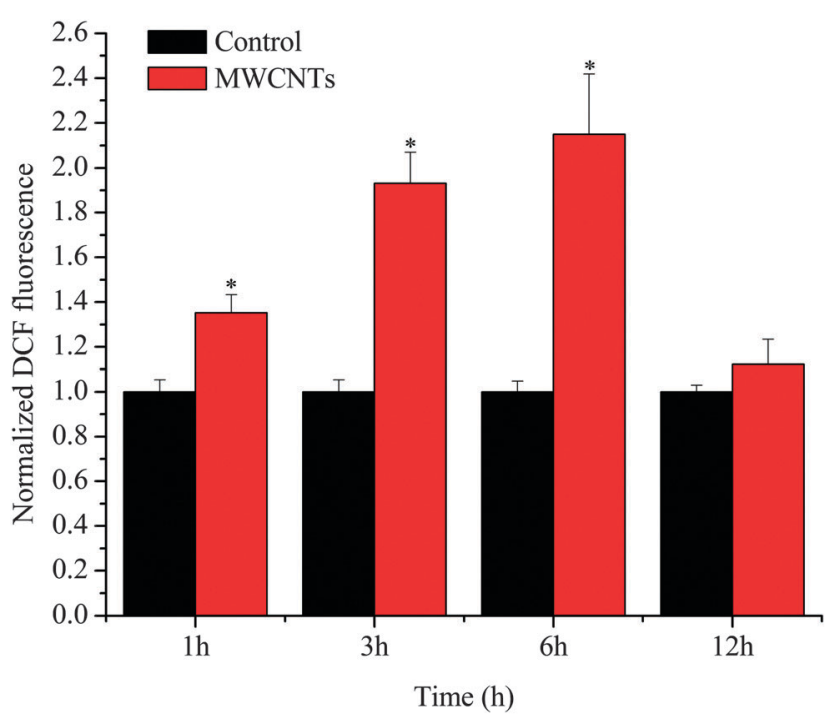

Fig. 3 Quantification of the intracellular levels of reactive oxygen species (ROS) generated upon exposure of BEAS-2B cells to $24 \mu \mathrm{g} \mathrm{cm}^{-2}$ MWCNTs. Intracellular ROS generation was determined using the oxidation-sensitive fluorescent probe $2^{\prime}, 7^{\prime}$-dichlorofluorescein diacetate and measuring the fluorescent intensity of control and MWCNTs exposed cells at 485 excitation and 520 emission. Results are considered significant for $p^{\star}<0.05$. high sensitivity of the DCF-DA fluorescent probe which can record $\mathrm{O}_{2}, \mathrm{OH}^{-}$, or $\mathrm{H}_{2} \mathrm{O}_{2}$ at very low concentrations even after a reduced uptake of the nanotubes; this is in contrast with the FACS sensitivity which requires higher FITC signal for detection. ${ }^{74}$ The drop after $12 \mathrm{~h}$ of exposure is presumably associated either with the regulations in the intracellular glutathione (GSH), a major antioxidant that protects cells from oxidative stress ${ }^{75}$ or the quenching effects of the internalized MWCNTs. ${ }^{76}$ In particular, prolonged MWCNTs exposure could lead to a gradual accumulation of GSH which will eventually balance the cellular ROS oxidative levels. ${ }^{77,78}$ Complementary, intracellular accumulation of MWCNTs could lead to quenching of the cell-derived radicals by scavenging of the O-centered molecules (i.e., $\mathrm{HO}^{-}$and $\mathrm{O}_{3}{ }^{+}$). ${ }^{79}$ Controlled experiments of the ROS levels of the cells exposed to purified MWCNTs relative to pristine counterparts are also shown (ESI, $\dagger$ Fig. S1). Results showed that the ROS levels for the cells exposed to pristine MWCNTs followed a similar trend to that of the cells exposed to their purified counterparts. The ROS levels for the cells exposed to pristine MWCNTs was significantly higher than that of control cells within the first hour of exposure, reached a maximum value after approximately $8 \mathrm{~h}$ of exposure and eventually decreased with time remaining yet significantly higher than controls even after longer exposure duration (i.e., $12 \mathrm{~h}$ ). Cross-comparison of the ROS levels between the cells exposed to pristine and purified MWCNTs respectively, showed that the pristine samples induced significantly higher ROS levels relative to their purified counterparts, for all time points being investigated. This observation is presumably associated either with the larger aspect-ratio of the pristine MWCNTs (as confirmed by AFM), their increased levels of metal impurities, (i.e., Fe; ${ }^{49,73,80,81}$ as confirmed by our EDX analysis), and/or lower dispersity (see dispersity analysis; Table S2, ESI $\dagger$ ).

Based on these results which showed both differential, timedependent uptake and intracellular ROS generation upon cells exposure to MWCNT, we hypothesize that MWCNTs accumulation in the cell leads to changes in cellular biomechanics, all in a time-dependent manner. Our hypothesis is supported by previous studies which showed that cells exposed to Fe-based nanoparticles generate ROS which induce changes in cell cytoskeleton and mechanical properties ${ }^{82}$ as well as our own previous studies which showed that exposure to MWCNTs for a determined time frame of $24 \mathrm{~h}$ induce changes in cellular biomechanics and nanotubes localization at the cell nucleus. ${ }^{26}$

The degree of cellular biomechanical changes upon 1, 3, 6 or $12 \mathrm{~h}$ of cellular exposure to MWCNTs were investigated using AFM and nanoindentation. ${ }^{26}$ Specifically, micro-scale cellular stiffness was assessed from the bending of the AFM cantilever upon tip indentation in response to a known applied force; fitting the force-indentation curve to the Hertz model provided quantitative measurements of both the cell body as well as cell nucleus as based on the interactions between the AFM tip and the cell. ${ }^{36}$ In particular, nuclear regions are known to lead to weaker engaging of the AFM tip relative to the cell edges. This is due to the less concentrated cytoskeleton fibers present at the nuclear region as well as the higher height of the nucleus relative to the cell edges. ${ }^{36,83}$ 
Analysis of the BEAS-2B cells exposed to MWCNTs for 1, 3, 6 or $12 \mathrm{~h}$ and fixed with glutaraldehyde is shown in Fig. 4; the fixation was chosen as a mean to preserve the cellular properties during tip scanning. ${ }^{26}$ Results indicated that control cells have Young's modulus ranging from 100 to $400 \mathrm{kPa}$ with higher values being recorded at the cell periphery (>600 $\mathrm{kPa}$ ) presumably due to the underlying plastic substrate. The nuclear region appeared softer when compared to the cellular body, showing Young's modulus values between 20-200 (Fig. 4a). The elastic modulus for both control and nanotubes exposed cells was about one magnitude higher than those of the live cells ${ }^{83,84}$ presumably due to the experimental conditions being used (i.e., glutaraldehyde fixation ${ }^{84}$ and/or AFM indentation approach performed at low speed). ${ }^{85}$

The average Young's modulus of unexposed cells and cells exposed to MWCNTs for different onsets are shown in Fig. 4b (whole cell) and Fig. 4c (cell nucleus) respectively. Analysis showed that 1 and $3 \mathrm{~h}$ exposures did not result in significant changes in the elastic moduli of the whole cells being studied however, $3 \mathrm{~h}$ exposure lead to significant changes in the Young's modulus of the nuclear regions of the exposed cells relative to their control counterparts. For longer exposure $(>6 \mathrm{~h})$, the elastic moduli of exposed cells (both whole cells and cells nuclei) showed a significant increase relative to their controls at the corresponding time points $\left(p^{*}<0.05\right)$, presumably because of the larger cellular accumulation of the uptaken nanotubes. ${ }^{32}$ Specially, after $6 \mathrm{~h}$, exposed cells showed an increase of about 39\% and $49 \%$ of their whole and nuclear Young's modulus respectively relative to their counterparts, i.e., unexposed cells. At $12 \mathrm{~h}$, exposed cells also showed an increase in their overall Young's modulus of about $22 \%$ and an increase of $28 \%$ in their nuclei Young's moduli both relative to their control counterparts. Such increases in Young moduli were significant and presumably due to the interactions of uptaken MWCNTs with cytoskeleton filaments like actin $^{86}$ or microtubules, ${ }^{35}$ or the DNA. ${ }^{72}$

The current study contributes to advancing understanding of both biochemical and biomechanical cellular transformations upon cellular exposure to MWCNTs with our analysis showing that early-induced cellular responses could be considered/have the potential to become adverse. In particular, our results emphasize the importance of studying the MWCNT-induced cell transformations at early onsets to understand the fundamental interactions of the uptaken MWCNTs with the cellular components, their cellular translocation and overall influence on the cellular fate. With the known affinity of the internalized MWCNTs for cytoskeletal elements, ${ }^{28,35,87}$ and cellular biomechanics ability to regulate homeostasis, cell proliferation, motility, and differentiation, ${ }^{88-90}$ subtle changes in the cellular mechanical properties can be correlated with phenotypical transformation and cancer initiation. ${ }^{91}$ While to our knowledge no previous studies have reported biomechanical cellular changes induced by exposure to MWCNTs at early onsets, our study is supported by a previous one showing that $24 \mathrm{~h}$ of exposure to MWCNTs induce changes in cellular biomechanics with nanotubes localization at the cell nucleus resulting in potential cell transformation to a malignant phenotype. ${ }^{26}$ The differential, time-dependent mechanical changes
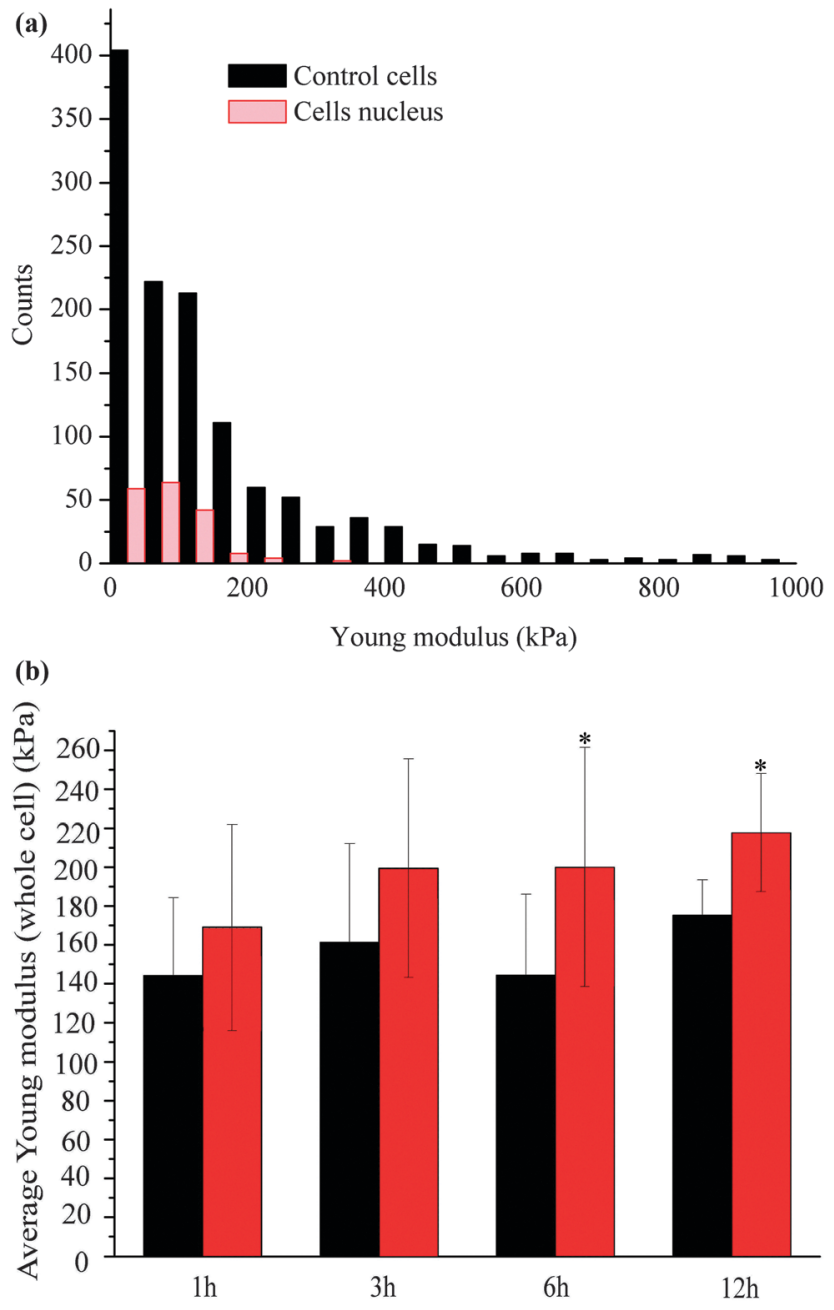

Cell treated with MWCNTs at different time points (h)

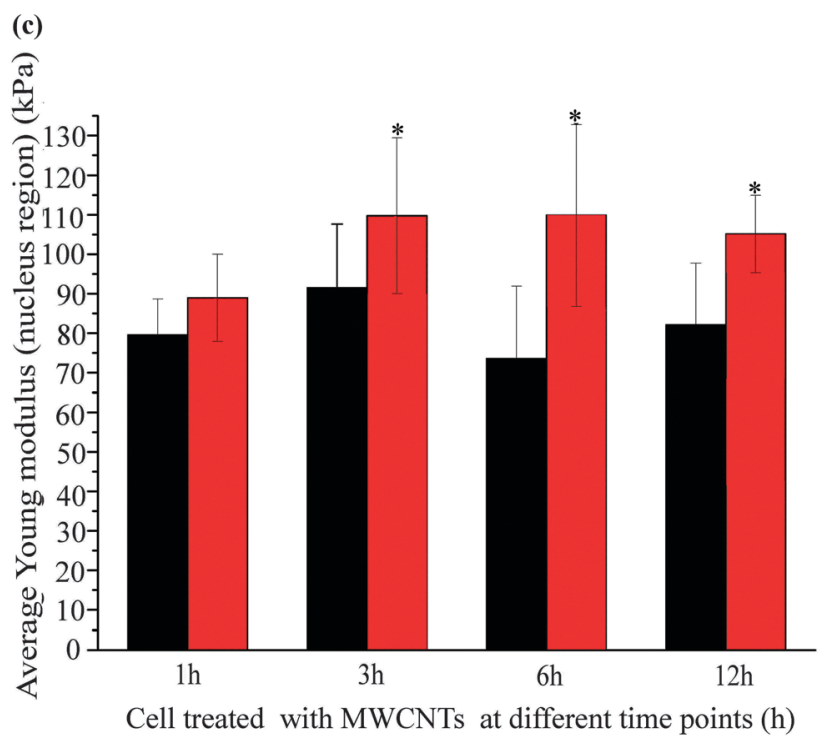

Fig. 4 (a) Histogram of the elastic modulus distribution of a representative control cell body and its nuclear region; (b) average elastic modulus of control cells and cells treated with MWCNTs $\left(24 \mu \mathrm{g} \mathrm{cm}^{-2}\right)$ at different time points (whole cell body); (c) average elastic modulus of nuclear regions of control cells and cells exposed to MWCNTs at different time points. Results are considered significant for $p^{*}<0.05$. 
observed upon early onsets cellular exposure could thus help prevent MWCNT-induced deleterious effects by reducing the risks for cell transformation. ${ }^{28,34,36}$

Further, our studies stress that in order to circumvent deleterious effects associated with nanomaterials exposure and to advance MWCNTs implementation in biomedical applications, one not only has to tightly control the exposure time but further to evaluate cellular changes and cellular ability to regain its homeostasis and how such processes are related to the differential time-dependent exposure to nanotubes. In particular, it is expected that assessment of early onsets of exposure derived from such studies would lead to more protective measurements than those based on direct adverse effects. For instance, such an assessment could provide a secondary level of prevention when exposure to MWCNTs is being considered $;^{92}$ by helping identify and distinguish early on the possible reactivities of the nanomaterials, methods to minimize their induced risks early in their design or manufacturing and down-stream user processes could be established.

\section{Conclusions}

The present study showed that exposure of human lung epithelial cells to occupationally relevant doses of user-characterized MWCNTs leads to their time-differential uptake, induced ROS generation and biomechanical cellular changes. In particular, our study showed that most of the MWCNTs uptake occurred within $6 \mathrm{~h}$ of cellular exposure. Furthermore, our results showed that $3 \mathrm{~h}$ exposure leads to changes in the cell elastic properties with further changes in the overall biomechanical properties being gradual and dependent on the exposure time. Assessment of the biomechanical properties of the exposed cells and their changes in elasticity suggest the ability to determine the underlying basis for MWCNT-induced cell transformation and toxicity using in vitro model cellular systems.

\section{Conflict of interests}

Research findings and conclusions are those of the authors and do not necessarily represent the views of the National Institute for Occupational Safety and Health. The authors declare no competing financial interest.

\section{Acknowledgements}

This work was supported by National Science Foundation (NSF) grant EPS-1003907, NSF 1434503, NSF 1300757, NORA 927000Y, and the National Institute of Health (NIH; R01-ES022968). The authors acknowledge use of WVU Shared Research Facilities, WVU Flow Cytometry Core Facility (supported by National Institute of Health equipment grant number S10OD016165 and the Institutional Development Award (IDeA) from the National Institute of General Medical Sciences of the National Institutes of Health under grant numbers P30GM103488 (CoBRE) and P20GM103434 (INBRE)). Authors also acknowledge the support and expertise of Dr Karen Martin, the director of the Core Facility, and help of Jeremy Hardinger with EDX analysis.

\section{References}

1 X. F. Zhang, Q. W. Li, T. G. Holesinger, P. N. Arendt, J. Y. Huang, P. D. Kirven, T. G. Clapp, R. F. DePaula, X. Z. Liao, Y. H. Zhao, L. X. Zheng, D. E. Peterson and Y. T. Zhu, Adv. Mater., 2007, 19, 4198-4201.

2 B. G. Demczyk, Y. M. Wang, J. Cumings, M. Hetman, W. Han, A. Zettl and R. O. Ritchie, Mater. Sci. Eng., A, 2002, 334, 173-178.

3 J. H. Zou, J. H. Liu, A. S. Karakoti, A. Kumar, D. Joung, Q. A. Li, S. I. Khondaker, S. Seal and L. Zhai, ACS Nano, 2010, 4, 7293-7302.

4 I. Stavarache, A. M. Lepadatu, V. S. Teodorescu, M. L. Ciurea, V. Iancu, M. Dragoman, G. Konstantinidis and R. Buiculescu, Nanoscale Res. Lett., 2011, 6, 88.

5 A. E. Aliev, M. H. Lima, E. M. Silverman and R. H. Baughman, Nanotechnology, 2010, 21, 035709.

6 S. W. Lee, B. S. Kim, S. Chen, Y. Shao-Horn and P. T. Hammond, J. Am. Chem. Soc., 2009, 131, 671-679.

7 M. F. L. De Volder, S. H. Tawfick, R. H. Baughman and A. J. Hart, Science, 2013, 339, 535-539.

8 O. Gohardani, M. C. Elola and C. Elizetxea, Prog. Aeronaut. Sci., 2014, 70, 42-68.

9 S. Dhall, N. Jaggi and R. Nathawat, Sens. Actuators, A, 2013, 201, 321-327.

10 K. Woan, G. Pyrgiotakis and W. Sigmund, Adv. Mater., 2009, 21, 2233-2239.

11 S. Mallakpour and A. Zadehnazari, Synth. Met., 2013, 169, 1-11.

12 M. S. Deshpande and S. Mazumdar, Biomacromolecules, 2012, 13, 1410-1419.

13 M. Gasser, B. Rothen-Rutishauser, H. F. Krug, P. Gehr, M. Nelle, B. Yan and P. Wick, J. Nanobiotechnol., 2010, 8, 31.

14 K. Bates and K. Kostarelos, Adv. Drug Delivery Rev., 2013, 65, 2023-2033.

15 S. Y. Madani, N. Naderi, O. Dissanayake, A. Tan and A. M. Seifalian, Int. J. Nanomed., 2011, 6, 2963-2979.

16 J. J. Khandare, A. Jalota-Badhwar, S. D. Satavalekar, S. G. Bhansali, N. D. Aher, F. Kharas and S. S. Banerjee, Nanoscale, 2012, 4, 837-844.

17 H. Ali-Boucetta, K. T. Al-Jamal, D. McCarthy, M. Prato, A. Bianco and K. Kostarelos, Chem. Commun., 2008, 459-461, DOI: $10.1039 / \mathrm{B} 712350 \mathrm{~g}$.

18 D. W. Porter, A. F. Hubbs, R. R. Mercer, N. Q. Wu, M. G. Wolfarth, K. Sriram, S. Leonard, L. Battelli, D. SchweglerBerry, S. Friend, M. Andrew, B. T. Chen, S. Tsuruoka, M. Endo and V. Castranova, Toxicology, 2010, 269, 136-147.

19 S. K. Sohaebuddin, P. T. Thevenot, D. Baker, J. W. Eaton and L. P. Tang, Part. Fibre Toxicol., 2010, 7, 22.

20 R. B. Li, X. Wang, Z. X. Ji, B. B. Sun, H. Y. Zhang, C. H. Chang, S. J. Lin, H. Meng, Y. P. Liao, M. Y. Wang, Z. X. Li, A. A. Hwang, T. B. Song, R. Xu, Y. Yang, J. I. Zink, A. E. Nel and T. Xia, ACS Nano, 2013, 7, 2352-2368. 
21 Y. Taquahashi, Y. Ogawa, A. Takagi, M. Tsuji, K. Morita and J. Kanno, J. Toxicol. Sci., 2013, 38, 619-628.

22 H. Nagai, Y. Okazaki, S. H. Chew, N. Misawa, Y. Yamashita, S. Akatsuka, T. Ishihara, K. Yamashita, Y. Yoshikawa, H. Yasui, L. Jiang, H. Ohara, T. Takahashi, G. Ichihara, K. Kostarelos, Y. Miyata, H. Shinohara and S. Toyokuni, Proc. Natl. Acad. Sci. U. S. A., 2011, 108, E1330-E1338.

23 Q. X. Mu, D. L. Broughton and B. Yan, Nano Lett., 2009, 9, 4370-4375.

24 S.-F. Ye, Y.-H. Wu, Z.-Q. Hou and Q.-Q. Zhang, Biochem. Biophys. Res. Commun., 2009, 379, 643-648.

25 D. van Berlo, V. Wilhelmi, A. W. Boots, M. Hullmann, T. A. Kuhlbusch, A. Bast, R. P. Schins and C. Albrecht, Arch. Toxicol., 2014, 88, 1725-1737.

26 C. B. Dong, M. L. Kashon, D. Lowry, J. S. Dordick, S. H. Reynolds, Y. Rojanasakul, L. M. Sargent and C. Z. Dinu, Adv. Healthcare Mater., 2013, 2, 945-951.

27 M. Plodinec, M. Loparic, C. A. Monnier, E. C. Obermann, R. Zanetti-Dallenbach, P. Oertle, J. T. Hyotyla, U. Aebi, M. Bentires-Alj, R. Y. H. Lim and C. A. Schoenenberger, Nat. Nanotechnol., 2012, 7, 757-765.

28 K. J. Siegrist, S. H. Reynolds, M. L. Kashon, D. T. Lowry, C. B. Dong, A. F. Hubbs, S. H. Young, J. L. Salisbury, D. W. Porter, S. A. Benkovic, M. McCawley, M. J. Keane, J. T. Mastovich, K. L. Bunker, L. G. Cena, M. C. Sparrow, J. L. Sturgeon, C. Z. Dinu and L. M. Sargent, Part. Fibre Toxicol., 2014, 11, 6.

29 D. Gavello, I. Fenoglio, B. Fubini, F. Cesano, F. Premoselli, A. Renna, E. Carbone and V. Carabelli, Neurotoxicology, 2013, 39, 84-94.

30 Z. B. Liu, X. Dong, L. P. Song, H. L. Zhang, L. X. Liu, D. W. Zhu, C. X. Song and X. G. Leng, J. Biomed. Mater. Res., Part A, 2014, 102, 665-673.

31 C. B. Dong, A. S. Campell, R. Eldawud, G. Perhinschi, Y. Rojanasakul and C. Z. Dinu, Appl. Surf. Sci., 2013, 264, 261-268.

32 S. Hirano, Y. Fujitani, A. Furuyama and S. Kanno, Toxicol. Appl. Pharmacol., 2010, 249, 8-15.

33 M. Asakura, T. Sasaki, T. Sugiyama, M. Takaya, S. Koda, K. Nagano, H. Arito and S. Fukushima, J. Occup. Health, 2010, 52, 9-20.

34 L. Sargent, S. Reynolds, D. Lowry, M. Kashon, S. Benkovic, J. Salisbury, A. Hubbs, M. Keane, J. Mastovich and K. Bunker, Proc. Am. Assoc. Cancer Res., 2012, 53, 1320.

35 L. Rodriguez-Fernandez, R. Valiente, J. Gonzalez, J. C. Villegas and M. L. Fanarraga, ACS Nano, 2012, 6, 6614-6625.

36 C. Dong, R. Eldawud, L. M. Sargent, M. L. Kashon, D. Lowry, Y. Rojanasakul and C. Z. Dinu, Environ. Sci.: Nano, 2014, 1, 95-603.

37 M. Foldvari and M. Bagonluri, Nanomedicine, 2008, 4, 183-200.

38 A. S. Campbell, C. Dong, F. Meng, J. Hardinger, G. Perhinschi, N. Wu and C. Z. Dinu, ACS Appl. Mater. Interfaces, 2014, 6, 5393-5403.

39 A. S. Campbell, C. Dong, J. S. Dordick and C. Z. Dinu, Process Biochem., 2013, 48, 1355-1360.
40 X. C. Zhao, R. T. Liu, Z. X. Chi, Y. Teng and P. F. Qin, J. Phys. Chem. B, 2010, 114, 5625-5631.

41 A. Gomes, E. Fernandes and J. L. F. C. Lima, J. Biochem. Biophys. Methods, 2005, 65, 45-80.

42 C. Rotsch, K. Jacobson and M. Radmacher, Proc. Natl. Acad. Sci. U. S. A., 1999, 96, 921-926.

43 A. B. Mathur, G. A. Truskey and W. M. Reichert, Biophys. J., 2000, 78, 1725-1735.

44 M. Radmacher, M. Fritz, C. M. Kacher, J. P. Cleveland and P. K. Hansma, Biophys. J., 1996, 70, 556-567.

45 V. M. Laurent, S. Kasas, A. Yersin, T. E. Schaffer, S. Catsicas, G. Dietler, A. B. Verkhovsky and J. J. Meister, Biophys. J., 2005, 89, 667-675.

46 T. G. Kuznetsova, M. N. Starodubtseva, N. I. Yegorenkov, S. A. Chizhik and R. I. Zhdanov, Micron, 2007, 38, 824-833. 47 B. Ohler, Rev. Sci. Instrum., 2007, 78, 063701.

48 S. Luanpitpong, L. Wang, V. Castranova and Y. Rojanasakul, Part. Fibre Toxicol., 2014, 11, 22.

49 V. E. Kagan, Y. Y. Tyurina, V. A. Tyurin, N. V. Konduru, A. I. Potapovich, A. N. Osipov, E. R. Kisin, D. SchweglerBerry, R. Mercer, V. Castranova and A. A. Shvedova, Toxicol. Lett., 2006, 165, 88-100.

50 T. A. Saleh, Appl. Surf. Sci., 2011, 257, 7746-7751.

51 K. Kostarelos, L. Lacerda, G. Pastorin, W. Wu, S. Wieckowski, J. Luangsivilay, S. Godefroy, D. Pantarotto, J. P. Briand, S. Muller, M. Prato and A. Bianco, Nat. Nanotechnol., 2007, 2, 108-113.

52 C. Z. Dinu, G. Zhu, S. S. Bale, G. Anand, P. J. Reeder, K. Sanford, G. Whited, R. S. Kane and J. S. Dordick, Adv. Funct. Mater., 2010, 20, 392-398.

53 C. Bertulli, H. J. Beeson, T. Hasan and Y. Y. Huang, Nanotechnology, 2013, 24, 265102.

54 B. D. Holt, K. N. Dahl and M. F. Islam, Small, 2011, 7, 2348-2355.

55 N. Grover, I. V. Borkar, C. Z. Dinu, R. S. Kane and J. S. Dordick, Enzyme Microb. Technol., 2012, 50, 271-279.

56 S. Vardharajula, S. Z. Ali, P. M. Tiwari, E. Eroğlu, K. Vig, V. A. Dennis and S. R. Singh, Int. J. Nanomed., 2012, 7, 5361-5374.

57 A. S. Campbell, C. Dong, A. Maloney, J. Hardinger, X. Hu, F. Meng, A. Guiseppe-Elie, N. Wu and C. Z. Dinu, Nano LIFE, 2014, 04, 1450005.

58 J. B. Griffiths, Dev. Biol. Stand., 1987, 66, 155-160.

59 Z. Yang and H.-R. Xiong, Culture Conditions and Types of Growth Media for Mammalian Cells, 2012.

60 L. Bokobza and J. Zhang, eXPRESS Polym. Lett., 2012, 6, 601-608.

61 S. N. Bokova, E. D. Obraztsova, V. V. Grebenyukov, K. V. Elumeeva, A. V. Ishchenko and V. L. Kuznetsov, Phys. Status Solidi B, 2010, 247, 2827-2830.

62 C. S. Hu, C. H. Chiang, P. D. Hong and M. K. Yeh, Int. J. Nanomed., 2012, 7, 4861-4872.

63 S. H. Lecker, A. L. Goldberg and W. E. Mitch, J. Am. Soc. Nephrol., 2006, 17, 1807-1819.

64 B. Kang, S. Chang, Y. Dai, D. Yu and D. Chen, Small, 2010, 6, 2362-2366. 
65 D. A. Tsyboulski, J.-D. R. Rocha, S. M. Bachilo, L. Cognet and R. B. Weisman, Nano Lett., 2007, 7, 3080-3085.

66 L. Cognet, D. A. Tsyboulski, J.-D. R. Rocha, C. D. Doyle, J. M. Tour and R. B. Weisman, Science, 2007, 316, 1465-1468.

67 V. Neves, E. Heister, S. Costa, C. Tîlmaciu, E. Borowiak-Palen, C. E. Giusca, E. Flahaut, B. Soula, H. M. Coley, J. McFadden and S. R. P. Silva, Adv. Funct. Mater., 2010, 20, 3272-3279.

68 C. F. Labuschagne and A. B. Brenkman, Ageing Res. Rev., 2013, 12, 918-930.

69 P. Ravichandran, A. Periyakaruppan, B. Sadanandan, V. Ramesh, J. C. Hall, O. Jejelowo and G. T. Ramesh, J. Biochem. Mol. Toxicol., 2009, 23, 333-344.

70 M. Pacurari, Y. Qian, W. Fu, D. Schwegler-Berry, M. Ding, V. Castranova and N. L. Guo, J. Toxicol. Environ. Health, Part A, 2012, 75, 129-147.

71 A. Manke, L. Wang and Y. Rojanasakul, BioMed Res. Int., 2013, 2013, 942916.

72 M. Pacurari, X. J. Yin, M. Ding, S. S. Leonard, D. SchweglerBerry, B. S. Ducatman, M. Chirila, M. Endo, V. Castranova and V. Vallyathan, Nanotoxicology, 2008, 2, 155-170.

73 A. Manke, S. Luanpitpong, C. Dong, L. Wang, X. He, L. Battelli, R. Derk, T. A. Stueckle, D. W. Porter, T. Sager, H. Gou, C. Z. Dinu, N. Wu, R. R. Mercer and Y. Rojanasakul, Int. J. Mol. Sci., 2014, 15, 7444-7461.

74 E. Eruslanov and S. Kusmartsev, Methods Mol. Biol., 2010, 594, 57-72.

75 F. Thayyullathil, S. Chathoth, A. Hago, M. Patel and S. Galadari, Free Radicals Biol. Med., 2008, 45, 1403-1412.

76 P. Boldog, K. Hajdu, M. Magyar, E. Hideg, K. Hernadi, E. Horvath, A. Magrez, K. Nagy, G. Varo, L. Forro and L. Nagy, Phys. Status Solidi B, 2013, 250, 2539-2543.

77 M. Luo, X. Y. Deng, X. Z. Shen, L. Dong and Y. F. Liu, J. Nanosci. Nanotechnol., 2012, 12, 274-283.
78 Y.-Y. Guo, J. Zhang, Y.-F. Zheng, J. Yang and X.-Q. Zhu, Mutat. Res., Genet. Toxicol. Environ. Mutagen., 2011, 721, 184-191.

79 I. Fenoglio, M. Tomatis, D. Lison, J. Muller, A. Fonseca, J. B. Nagy and B. Fubini, Free Radicals Biol. Med., 2006, 40, 1227-1233.

80 Y. Sato, A. Yokoyama, K. Shibata, Y. Akimoto, S. Ogino, Y. Nodasaka, T. Kohgo, K. Tamura, T. Akasaka, M. Uo, K. Motomiya, B. Jeyadevan, M. Ishiguro, R. Hatakeyama, F. Watari and K. Tohji, Mol. BioSyst., 2005, 1, 176-182.

81 E. Aldieri, I. Fenoglio, F. Cesano, E. Gazzano, G. Gulino, D. Scarano, A. Attanasio, G. Mazzucco, D. Ghigo and B. Fubini, J. Toxicol. Environ. Health, Part A, 2013, 76, 1056-1071.

82 K. Buyukhatipoglu and A. M. Clyne, J. Biomed. Mater. Res., Part A, 2011, 96, 186-195.

83 F. Rico, J. Alcaraz, J. J. Fredberg and D. Navajas, Int. J. Nanotechnol., 2005, 2, 180-194.

84 J. Rheinlaender, N. A. Geisse, R. Proksch and T. E. Schaffer, Langmuir, 2011, 27, 697-704.

85 D. P. Juan, P. Ruiz, J. Dias, N. M. Ziebarth and H. S Cheung, Cell Health Cytoskeleton, 2012, 4, 29-35.

86 B. D. Holt, P. A. Short, A. D. Rape, Y. L. Wang, M. F. Islam and K. N. Dahl, ACS Nano, 2010, 4, 4872-4878.

87 B. N. Snyder-Talkington, M. Pacurari, C. L. Dong, S. S. Leonard, D. Schwegler-Berry, V. Castranova, Y. Qian and N. L. Guo, Toxicol. Sci., 2013, 133, 79-89.

88 I. Titushkin and M. Cho, Biophys. J., 2009, 96, 717-728.

89 F. C. Su, C. C. Wu and S. Chien, J. Med. Biol. Eng., 2011, 31, 233-244.

90 J. Galle, A. Krinner, P. Buske, D. Drasdo and M. Loeffler, IFMBE Proc., 2009, 25, 185-188.

91 S. E. Cross, Y. S. Jin, J. Rao and J. K. Gimzewski, Nat. Nanotechnol., 2007, 2, 780-783.

92 M. M. Dahm, D. E. Evans, M. K. Schubauer-Berigan, M. E. Birch and J. A. Deddens, Ann. Occup. Hyg., 2013, 57, 328-344. 